\title{
Non-Canonical Amino Acids as Minimal Tags for Investigating Protein Organization and Turnover
}

\section{Dissertation}

in partial fulfilment of the requirements for the degree

"Doctor of Natural Sciences (Dr. rer. nat.)"

in the Molecular Biology Program

at the Georg August University Göttingen

Faculty of Biology

submitted by

\section{Ingrid-Cristiana Gebura-Vreja \\ born in}

Râmnicu-Vâlcea, Romania

Göttingen, September 2015 

Members of the Thesis Committee:

Supervisor, reviewer: $\quad$ Prof. Dr. Silvio O. Rizzoli

Department of Neuro- and Sensory Physiology

University Medical Center Göttingen, Germany

Reviewer:

Prof. Dr. Reinhard Jahn

Department of Neurobiology

Max Planck Institute for Biophysical Chemistry

Committee member: $\quad$ Prof. Dr. Blanche Schwappach

Department of Molecular Biology

University Medical Center Göttingen, Germany

Date of oral examination: October $14^{\text {th }}, 2015$ 

(Affidavit)

I hereby declare that I prepared the dissertation entitled "Non-Canonical Amino Acids as Minimal Tags for Investigating Protein Organization and Turnover" on my own and with no other sources and aids than quoted.

Ingrid-Cristiana Gebura-Vreja 

"Iar în lumea asta mare, noi copii ai lumii mici,

Facem pe pământul nostru muşunoaie de furnici;

Microscopice popoare, regi, oşteni şi învăţaţi

Ne succedem generaţii şi ne credem minunaţi;

$(\ldots)$

Precum pulberea se joacă în imperiul unei raze,

Mii de fire viorie ce cu raza încetează,

Astfel, într-a veciniciei noapte pururea adâncă,

Avem clipa, avem raza, care tot mai ţine încă...

Cum s-o stinge, totul piere, ca o umbră-n întuneric,

Căci e vis al nefiinţei universul cel himeric..."

Mihai Eminescu, Scrisoarea I, 1 februarie 1881

"while we, inheritors of space, the children of this world of awe, Are raising witless heaps of sand upon our little earthy floor;

Microscopic nations rise with warrior and king and seer,

Throughout the years our fortunes wax, until we have forgotten fear.

Just as the motes of dust enjoy their kingdom in the lamplight's ray,

Thousands specks that are no more when once that beam has passed away

So, in the midst of endless night, we have our little time to spend,

Our moment snatched from chaos, which did not yet come to an end.

But when our beam at last goes out, our world will suddenly disperse

Amidst the dark that ever hangs around this whirling universe."

Mihai Eminescu, Satire I, February $1^{\text {st }}, 1881$

Translated by Corneliu M. Popescu 



\section{TABLE OF CONTENTS}

TABLE OF CONTENTS ....................................................................................................ix

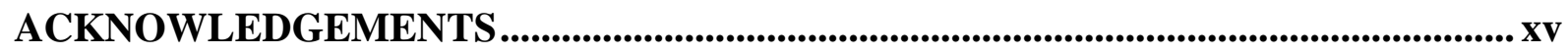

LIST OF PUBLICATIONS........................................................................................................

LIST OF FIGURES ......................................................................................................................xviii

LIST OF TABLES ............................................................................................................................ $\mathrm{xx}$

LIST OF EQUATIONS ....................................................................................................... $\mathrm{xx}$

LIST OF ABBREVIATIONS........................................................................................xi

ABSTRACT ....................................................................................................................................... XXv

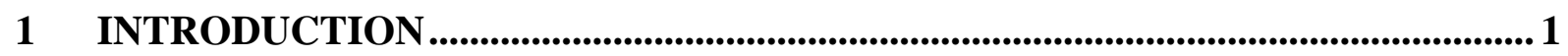

$1.1 \quad$ The Importance of Probe Size in Detecting Proteins ……………............................ 1

1.1.1 Genetically Encoded Tags ................................................................................ 3

1.2 Genetic Code Expansion and Its Applications ……………………………........ 5

1.2.1 General Protein Labeling ....................................................................... 6

1.2.2 Specific Protein Labeling ................................................................................ 6

1.3 Labeling Non-Canonical Amino Acids via Click Chemistry …………………......... 8

1.3.1 Copper-Catalyzed Alkyne-Azide Cycloaddition (CuAAC) ..................................

1.3.2 Strain-Promoted Alkyne-Azide Cycloaddition (SPAAC),.................................. 10

1.3.3 Strain-Promoted Inverse-Electron Demand Diels-Alder Cycloaddition

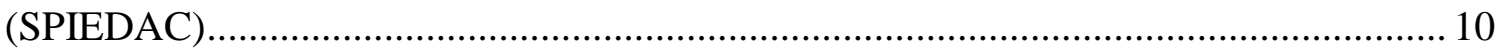

1.4 A Technical Perspective on Super-Resolution Microscopy …………........................ 11

1.4.1 Ensemble Methods: STED Nanoscopy ……………………………………...... 14 
1.4.2 Single Molecule Methods: GSDIM, STORM, PALM..................................... 16

1.5 A Technical Perspective on Secondary Ion Mass Spectrometry (SIMS) ................. 18

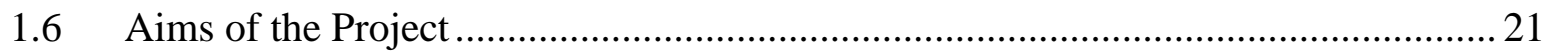

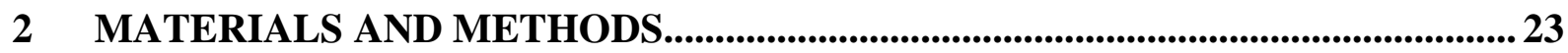

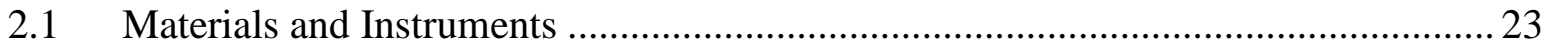

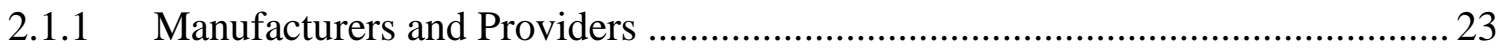

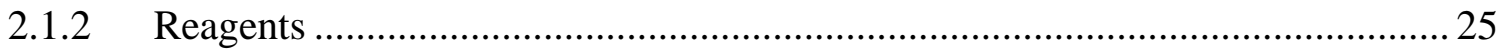

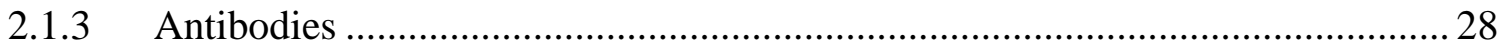

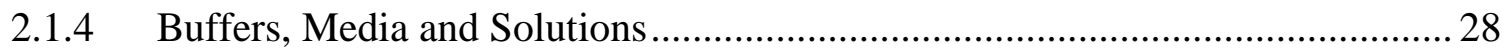

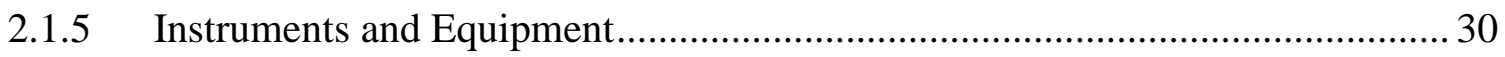

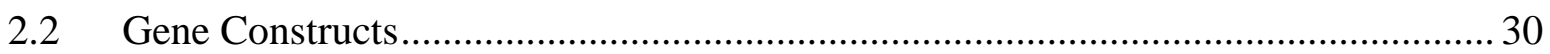

2.2.1 Constructs Obtained by Site-Directed Mutagenesis........................................ 33

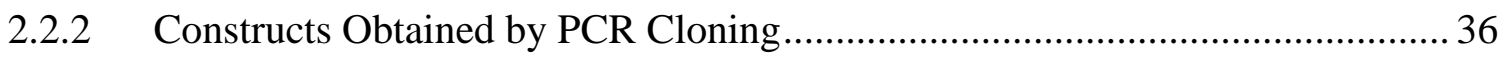

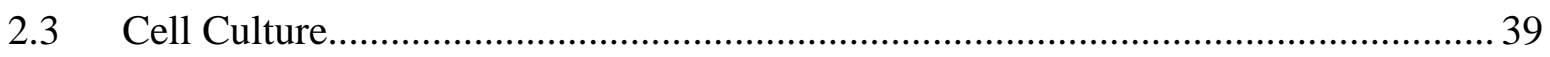

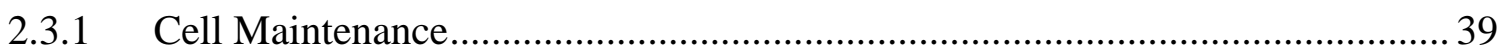

2.3.2 Plating Cells for Fluorescence Miscroscopy ............................................... 39

2.3.3 Plating Cells for Western Blotting …......................................................... 39

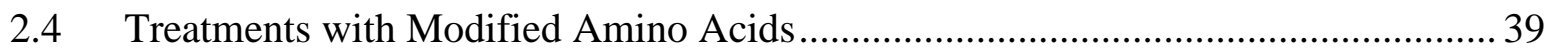

2.4.1 Clickable Non-Canonical Amino Acids............................................................. 39

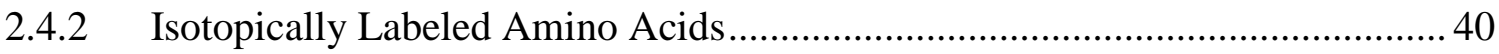

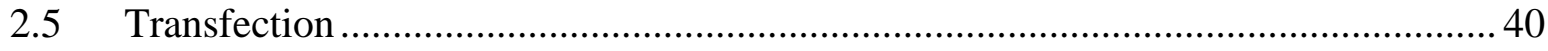

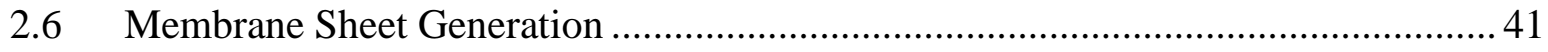

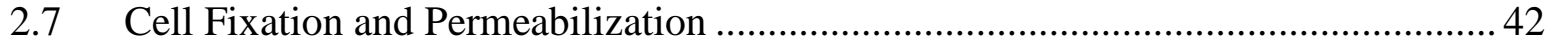

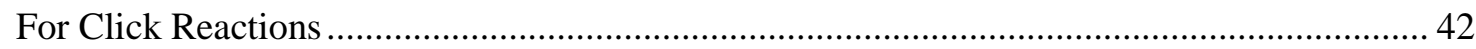

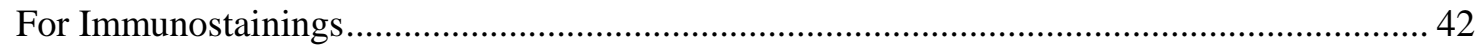

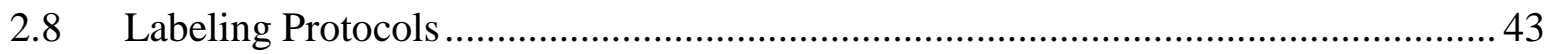




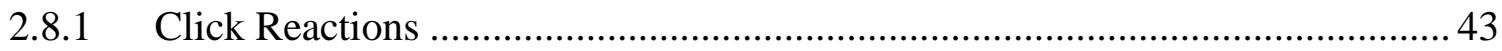

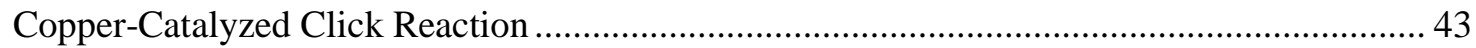

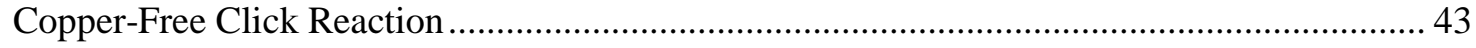

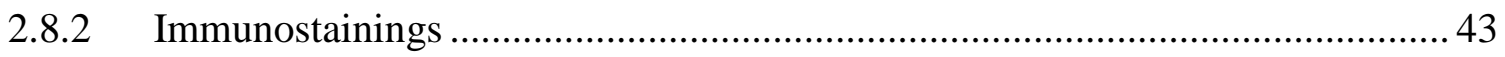

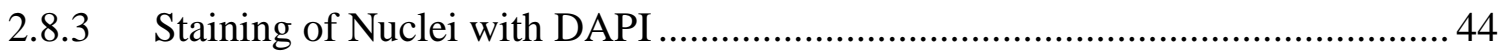

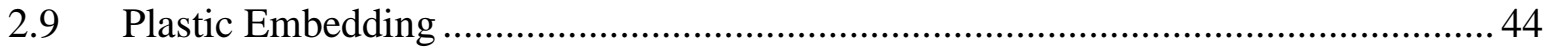

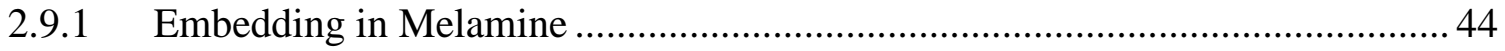

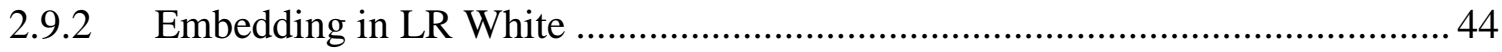

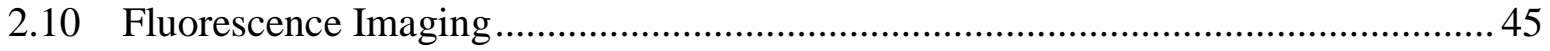

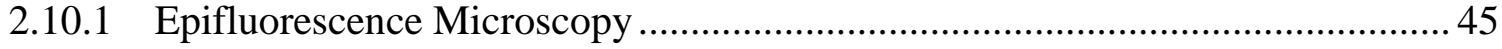

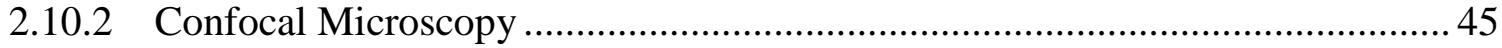

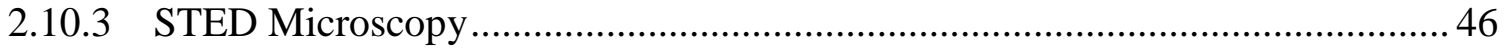

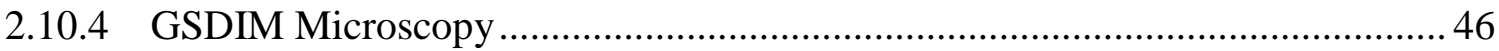

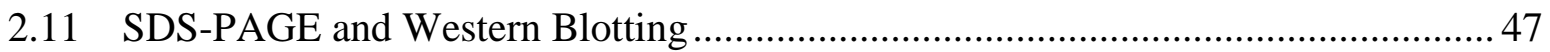

2.12 Sample Preparation for SPILL Measurements ........................................................... 47

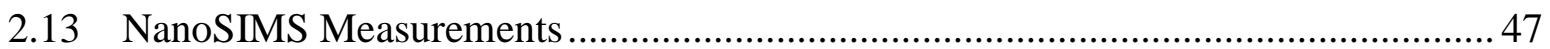

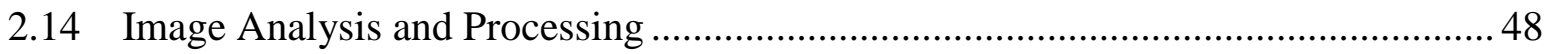

2.14.1 Data Analysis for Epifluorescence Images ................................................... 48

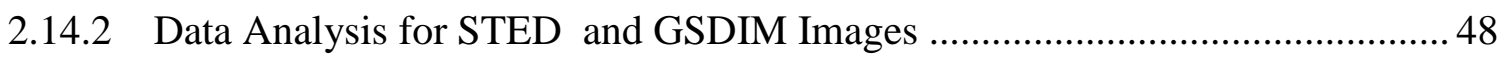

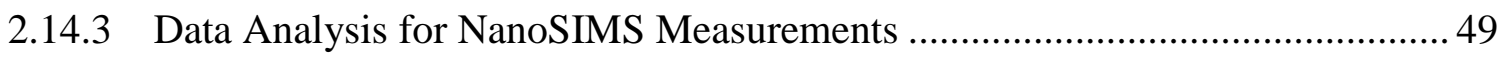

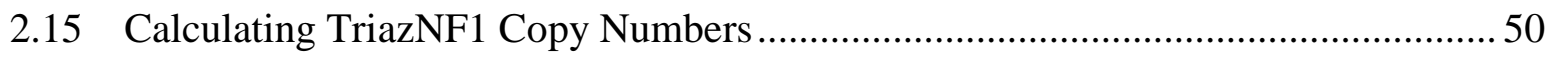

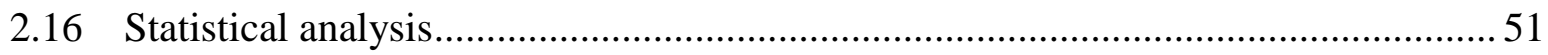

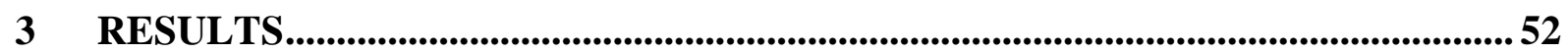

3.1 Constructs for Non-Canonical Amino Acid Encoding ....................................... 52

3.1.1 Constructs Obtained by Site-Directed Mutagenesis....................................... 52

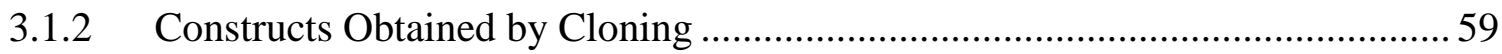




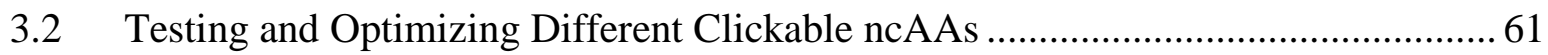

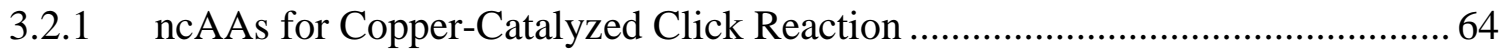

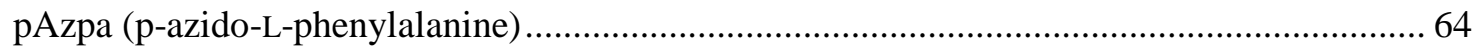

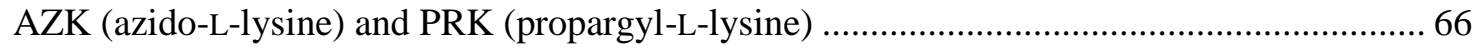

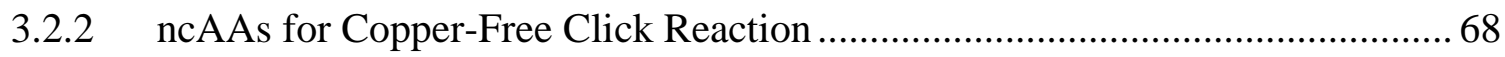

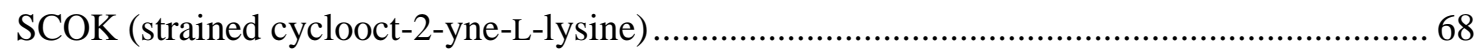

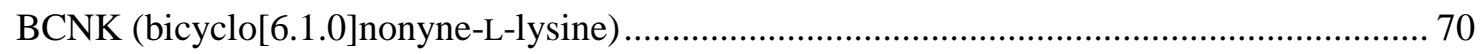

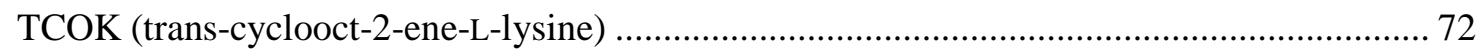

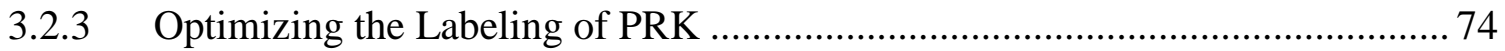

3.3 ClickOx as a Possible Tool to Reduce Oxidative Stress ......................................... 78

3.4 Incorporation of PRK into the Proteins of Interest .............................................. 80

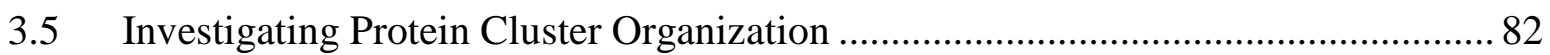

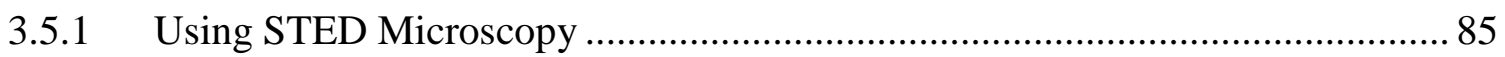

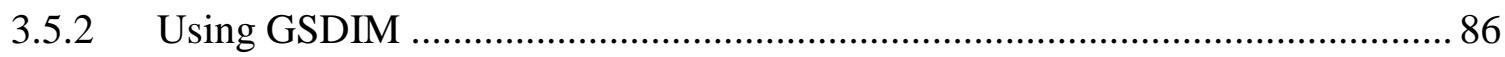

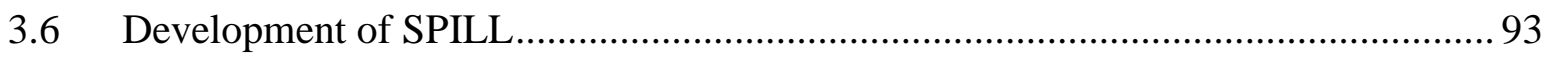

3.6.1 Testing Different Probes for Metabolic Investigations ................................... 94

3.6.2 Correlation between NanoSIMS Measurements and Confocal Imaging ........... 99

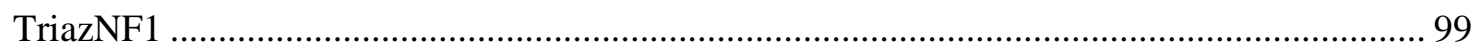

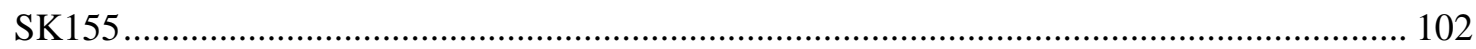

3.6.3 SPILL as a Tool to Investigate Specific Protein Turnover ............................. 105

4 DISCUSSION .................................................................................................................... 107

4.1 The Effect of FPs on the Protein Organization...................................................... 108

4.1.1 Implications on Studies Involving Fluorescent Proteins ............................... 109

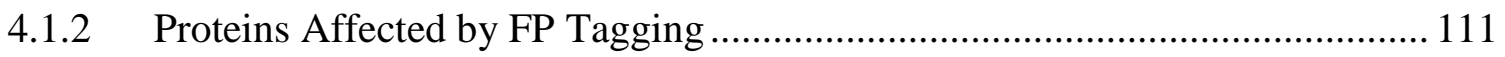

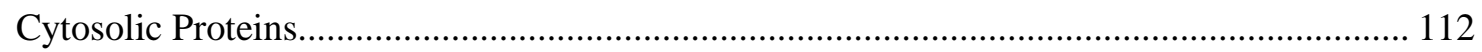

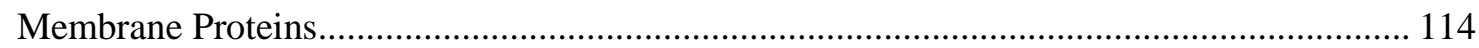




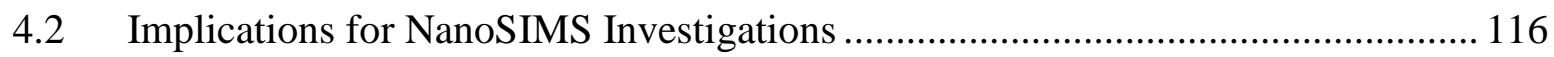

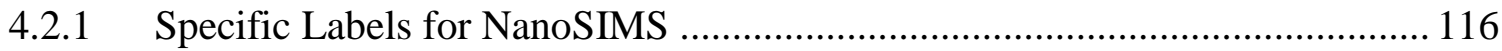

4.2.2 Specific Protein Turnover Revealed by SPILL ........................................... 117

4.3 The Experimental Approach and its Limitations............................................... 118

4.3.1 Non-Canonical Amino Acid Incorporation Technique .................................. 118

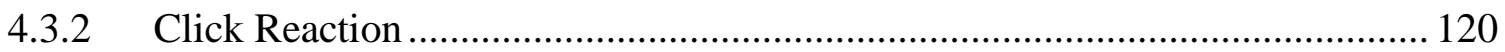

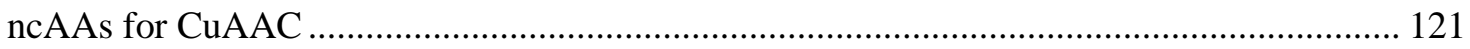

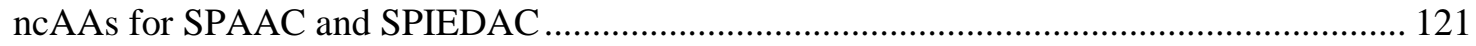

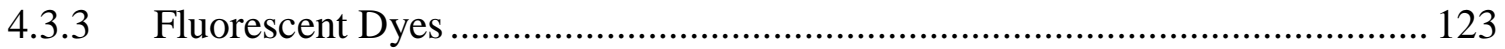

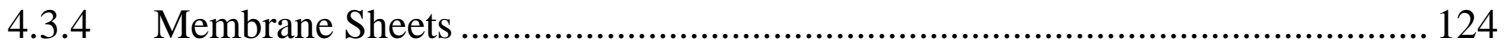

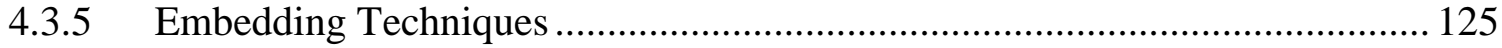

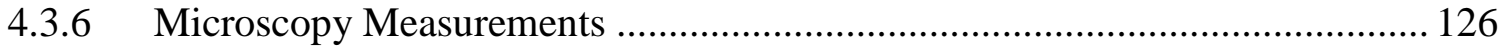

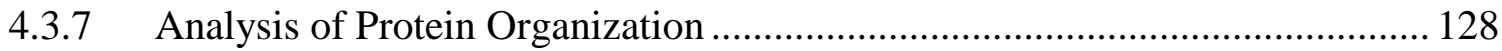

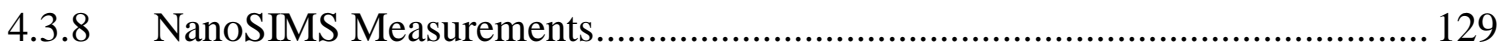

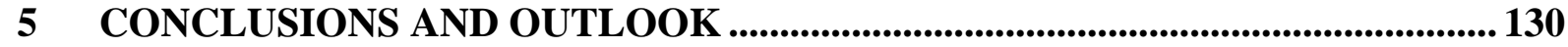

5.1 Labeling of Proteins for Super-Resolution Light Microscopy ............................... 131

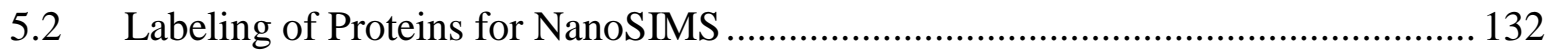

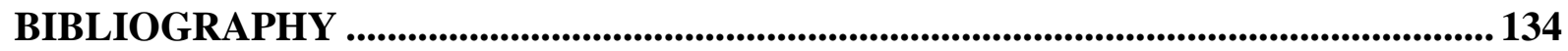

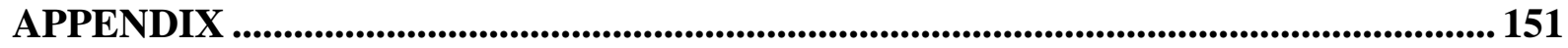

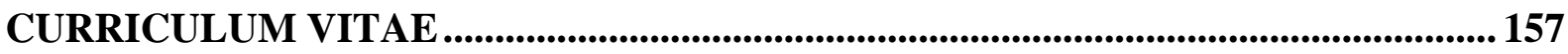





\section{ACKNOWLEDGEMENTS}

First and foremost, I would like to thank my supervisor Silvio Rizzoli for the excellent guidance as well as for opening my mind to the larger world of scientific research and the philosophy behind it. Thank you for encouraging me to see the bigger picture and to surpass my limits!

I would like to thank Reinhard Jahn and Blanche Schwappach for being part of my thesis committee and for their suggestions for my project. I am grateful to Claudia Höbartner, Stefan Jakobs and Michael Müller for taking part in my extended thesis committee.

To continue with, I would like to thank all the present and former members of the Rizzoli lab for sustaining such a wonderful work environment. I would especially like to thank Nora Wender, Sinem Saka-Kirli, Eugenio Fornasiero, Natalia Revelo-Nuncira, Felipe Opazo, Hanna Wildhagen, and Angela Gomes for the inspiring discussions and friendly help in science-related or broader matters. I would also like to express my special thanks to Katharina Kröhnert and Regina Sommer-Kluß for their patience in answering my bureaucracy-related questions. I am also indebted to Christina Schäfer, Katharina Kröhnert, and Gaby Klaehn, for their help with cell culture preparations, sample embedding and cutting, and with DNA purification, respectively. My sincere thanks are addressed to Burkhard Rammner who helped me with the 3D renditions of different molecules.

My gratitude extends to the collaborators with whose contribution and help this work has reached publication stage. I am very grateful to Stefan Hell and Stefan Jakobs for providing me access to their microscopy facilities. I also feel indebted to Fabian Göttfert who kindly instructed and allowed me to use his state-of-the-art STED setup as well as to Mark Bates who introduced me to GSDIM/STORM imaging. I would also like to thank Ulf Diederichsen and Selda Kabatas for synthesizing the organic compounds TriazNF1 and SK155, among others, which we have successfully tested and implemented in NanoSIMS imaging. Special thanks to Selda Kabatas for her help in deciphering the ChemDraw and Chemsketch 
software and for depicting part of the chemical formulas for this project. Sincere thanks go to Carmen Höschen and Hans Lugmeier for their assistance with NanoSIMS. In addition, I thank Marcus Niebert and Ivana Nikić for kindly giving me their Amber stop codon mutants. I am deeply indebted to Reinhard Jahn for the kind gift of over 20 plasmids from his library.

I would like to acknowledge the funding for my $\mathrm{PhD}$ project from a Dorothea Schlözer Fellowship provided by the University of Göttingen. I am also thankful for the GGNB and IMPRS MolBio travel grants that enabled me to attend inspiring scientific meetings.

I am greatly indebted to Steffen Burkhardt, Kerstin Grüniger, Kirsten Pöhlker, and Katrin Wodzicki for their amazing work in setting up and maintaining the International Max Plack Research School for Molecular Biology and the Göttingen Graduate School for Neurosciences, Biophysics, and Molecular Biosciences (GGNB) programs. I consider myself incredibly lucky to have had the opportunity to be a student in these programs and benefit from the inspiring courses and extra activities offered.

I would like to thank the wonderful friends I have met here in Göttingen: Maria Levchenko, Anita Smarandache, Ömer Cicek, Metin Aksu, Avani Shukla, Sona Pirkulyieva, and Jonas Barth who have spiced up my day-to-day life with witty jokes, discussions, and delicious dinners. I cannot thank enough my dear friend Maria, whose support has made me a stronger person and to whom I will forever be grateful for being there for me during the highs and the lows of writing this thesis.

Last but not least, I would like to thank my family for their constant support and encouragement to pursue my dreams, and especially my husband, Myroslav Gebura, who patiently and lovingly makes me a better person every day. 


\section{LIST OF PUBLICATIONS}

Parts of the results presented in this dissertation are included in the publications and manuscripts that follow:

Vreja, I.C.*, Kabatas, S.*, Saka, S.K., Kröhnert, K., Höschen, C., Diederichsen, U., Rizzoli, S.O. Secondary ion mass spectrometry of genetically-encoded targets. Angewandte Chemie International Edition, 2015, vol 54, pages 5784-5788

Kabatas, S.*, Vreja, I.C.*, Saka, S.K., Kröhnert, K., Höschen, C., Kröhnert, K., Opazo F., Rizzoli, S.O., Diederichsen, U. A contamination-insensitive probe for imaging specific biomolecules by secondary ion mass spectrometry. Chemical Communications, 2015, vol 51, pages $13221-13224$

Vreja, I.C., Nikić, I., Göttfert, F., Bates, M.W., Kröhnert K., Outeiro, T., Hell, S.W., Lemke E.A., Rizzoli, S.O. Super-resolution microscopy of clickable amino acids reveals the effects of fluorescent protein tagging on protein assemblies. ACS Nano, 2015, vol 9, pages 1103411041

* Equal contribution 


\section{LIST OF FIGURES}

Figure 1-1 Size comparison for the different protein affinity probes and tags ........................... 2

Figure 1-2 Antibodies and derived probes................................................................................. 3

Figure 1-3 The green fluorescent protein (GFP) fluorophore.................................................. 4

Figure 1-4 The principle of ncAA incorporation ................................................................................... 7

Figure 1-5 The copper-catalyzed alkyne-azide cycloaddition (CuAAC)......................................9

Figure 1-6 The strain-promoted alkyne-azide cycloaddition (SPAAC) ..................................... 10

Figure 1-7 The strain-promoted inverse-electron demand Diels-Alder cycloaddition

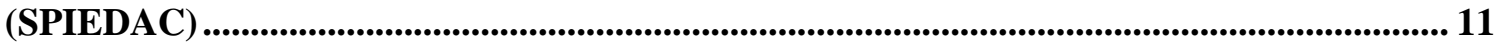

Figure 1-8 Absorption and emission of fluorescent dyes ..................................................................... 12

Figure 1-9 The STED nanoscopy principle........................................................................... 15

Figure 1-10 The principle behind super-resolution localization microscopy ................................. 17

Figure 1-11 The NanoSIMS setup and principle................................................................ 19

Figure 2-1 Experimental timeline for SPILL _......................................................................................... 40

Figure 2-2 The sonication setup................................................................................................................. 42

Figure 2-3 Parameters considered in data analysis from STED and GSDIM images............. 49

Figure 3-1 Constructs containing fluorescent protein (FP) chimeras with Amber stop codons introduced by site-directed mutagenesis ................................................................................................. 54

Figure 3-2 Testing ncAA incorporation using Western blotting ............................................... 56

Figure 3-3 Constructs without fluorescent protein (FP) tags.................................................5 58

Figure 3-4 Restriction maps for the VAMP2 pEGFP-N1 and pN1 vectors ...............................60

Figure 3-5 The structures for the ncAAs tested in this study.....................................................61

Figure 3-6 Structures for the fluorophores used in this project and putative other dyes....... 63

Figure 3-7 pAzpa click reaction results in low signal-to-noise levels ........................................... 65

Figure 3-8 AZK and PRK are well incorporated into VAMP2, but only PRK reacts efficiently in CuAAC

Figure 3-9 SCOK incorporation and click reaction reaction results in nonspecific labeling.. 69

Figure 3-10 BCNK incorporation and click chemistry shows unspecific labeling ...................... 71

Figure 3-11 No specific labeling in copper-free click reaction for BCNK, SCOK, and TCOK 73

Figure 3-12 Labeling specificity Atto647N-azide and KK114-azide ........................................... 76

Figure 3-13 Optimal labeling parameters using PRK and Star635P-azide .................................. 77

Figure 3-14 Similar labeling intensities for ClickOx and normal samples ................................... 79 
Figure 3-15 PRK is well incorporated in all 26 proteins included in this study

Figure 3-16 Genetic encoding of PRK and click reaction with azide derivatives for labeling protein assemblies for nanoscopy investigations.......................................................................... 82

Figure 3-17 Comparison of samples imaged in confocal versus STED ...................................... 84

Figure 3-18 The organization of SNAP-25, syntaxin 1, and $\alpha$-synuclein in STED microscopy 85

Figure 3-19 The organization of investigated proteins in GSDIM ............................................. 88

Figure 3-20 Comparison of mean spot size, peak and total spot intensity in GSDIM ............... 89

Figure 3-21 Graphical depictions of the ratios between the FP-tagged proteins versus the non-

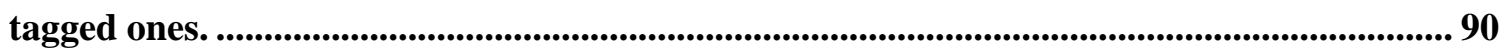

Figure 3-22 $\mathbf{k}_{\text {means }}$ cluster analysis.............................................................................................................. 92

Figure 3-23 The specific protein isotopic and fluorescence labeling (SPILL) technique .......... 93

Figure 3-24 Specificity of different high-content nitrogen compounds in click reactions......... 95

Figure 3-25 Probes for SPILL labeling: molecular structures of TriazNF1 and SK155.......... 96

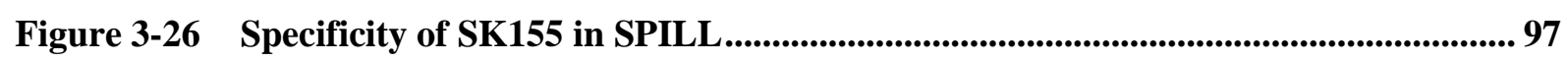

Figure 3-27 Sample preparation for confocal and NanoSIMS imaging......................................98

Figure 3-28 TriazNF1 specificity in NanoSIMS and confocal imaging..................................... 100

Figure 3-29 Specificity and quantitation of the TriazNF1 signal in NanoSIMS ...................... 101

Figure 3-30 SK155 specifically labels the proteins of interest for NanoSIMS measurements 103

Figure 3-31 NanoSIMS resolution and correlation with confocal imaging ............................... 104

Figure 3-32 Visualizing specific protein metabolism using SPILL............................................. 105

Figure 3-33 ${ }^{19} \mathrm{~F}$ offers more reliable correlation with turnover than fluorescence .................... 106

Figure 4-1 Overview of significance in t-tests for the investigated proteins ........................... 112 


\section{LIST OF TABLES}

Table 2-1 Companies and institutions that have provided the materials and instruments ... 23

Table 2-2 List of chemicals .................................................................................................... 25

Table 2-3 Primary and secondary antibodies used in blotting and cell stainings.................... 28

Table 2-4 Buffers, media and solutions used in this project ............................................................. 28

Table 2-5 List of instruments and devices ........................................................................................... 30

Table 2-6 Original constructs, their reference sequence, organism of origin, and source..... 31

Table 2-7 List of Amber mutants obtained through site-directed mutagenesis........................ 34

Table 2-8 List of Amber mutants obtained through PCR cloning ................................................ 37

Table 2-9 Plasmid systems with the RS/tRNA pair for ncAA incorporation.............................. 41

Table 2-10 Filter sets used for the Olympus epifluorescence microscope ................................... 45

Table 3-1 Summary for the click reaction specificity for the tested ncAAs ............................... 74

Table A-1 The sizes* of the molecules in Figure 1-1 ......................................................... 151

Table A-2 Summary of investigated proteins that showed significant differences*............... 153

Table A-3 Fluorescence properties for different far-red dyes................................................. 154

\section{LIST OF EQUATIONS}

Equation 1. Abbe's formula for the resolution attained in the xy plane................................ 13

Equation 2. Numerical aperture (NA) formula.............................................................................. 13

Equation 3. Resolution formula for STED microscopy ....................................................... 14

Equation 4. Localization accuracy formula for pointillistic nanoscopy methods .................... 17

System of Equations 5. Calculation of TriazNF1 copy numbers .................................................... 50

Equation 6. Derived TriazNF1 copy number formula................................................................50 


\section{LIST OF ABBREVIATIONS}

\begin{tabular}{|c|c|}
\hline $\mathrm{AcOH}$ & acetic acid \\
\hline AOBS & acousto-optical beam splitter \\
\hline AOTF & acousto-optical tunable filter \\
\hline APD & avalanche photodiode \\
\hline BAR & bridging interactor-amphiphysin-reduced viability upon starvation (domain) \\
\hline AZK & azido-L-lysine \\
\hline AHA & L-azidohomoalanine \\
\hline $\mathrm{AU}$ & arbitrary units \\
\hline BAR & Bin-Amphiphysin-Rvs (domain) \\
\hline BCNK & bicyclo[6.1.0]nonyne-L-lysine \\
\hline BHK & baby hamster kidney (cell line) \\
\hline BSA & bovine serum albumin \\
\hline CMV & cytomegalovirus \\
\hline $\mathrm{CuAAC}$ & copper(I)-catalyzed alkyne-azide cycloaddition \\
\hline DAPI & 4',6'-diamino-2-phenylindole $2 \mathrm{HCl}$ \\
\hline DIV & days in vitro \\
\hline DMEM & Dulbecco's Modified Eagle Medium \\
\hline $\operatorname{Doc} 2 \alpha$ & double C2-like domains, alpha \\
\hline dSTORM & direct stochastic optical reconstruction microscopy \\
\hline EGFP & enhanced green fluorescent protein \\
\hline EGTA & ethylene glycol tetraacetic acid \\
\hline eRF-1/ETF1 & eukaryotic release factor 1 / eukaryotic translation termination factor 1 \\
\hline $\mathrm{Fab}$ & antigen-binding fragment \\
\hline $\mathrm{Fc}$ & crystallizable fragment \\
\hline FCS & fetal calf serum \\
\hline FlAsH & fluorescein arsenical helix binder \\
\hline FP & fluorescent protein \\
\hline
\end{tabular}


FWHM full width at half maximum

FPALM fluorescence photo-activation localization microscopy

Gag group-specific antigen

GFP green fluorescent protein

GPI glycosylphosphatidylinositol

GRO genomically recoded organism

GSD ground state depletion (microscopy)

GSDIM ground state depletion followed by individual molecule return

HEPES 4-(2-hydroxyethyl)-1-piperazineethansulfonic acid

HIV human immunodeficiency virus

HPG L-homo-propargylglycine

5HT1a 5-hydroxytryptamine receptor 1a

IgG immunoglobulin $\mathrm{G}$

InsR insulin receptor

KAc potassium acetate

KGlu monopotassium glutamate

$\lambda_{\text {abs }} \quad$ wavelength for maximum absorbtion

$\lambda_{\mathrm{em}} \quad$ wavelength for maximum emission

LP long pass

$\beta$-ME $\quad \beta$-mercaptoethanol

MEA mercaptoethylamine

NA numerical aperture

NAC non-amyloid- $\beta$ component

NanoSIMS nanoscale secondary ion mass spectrometry

ncAA non-canonical amino acid

NMR nuclear magnetic resonance

NSF N-ethylmaleimide-sensitive factor/N-ethylmaleimide sensitive fusion protein

PALM photo-activation localization microscopy

pAzpa 4-azido-L-phenylalanine

PBS phosphate buffered saline

PCR polymerase chain reaction

PDB protein data base

xxii 
PFA paraformaldehyde

PIP2 phosphatidylinositol 4,5-bisphosphate

PIPKI $\quad$ phosphatidylinositol-4-phosphate 5-kinase, type I, gamma

PLL poly-L-lysine

PMT photomultiplier tube

ppb parts per billion

PRK propargyl-L-lysine

PSF point spread function

Pyl pyrrolysine/pyrrolysyl

PylRS pyrrolysyl-tRNA synthetase

QY $\quad$ quantum yield

Rab Ras-related protein

$\mathrm{ReAsH} \quad$ resorufin arsenical helix binder

RefSeq reference sequence

ROI region of interest

ROS reactive oxygen species

rpm rotations per minute

RT room temperature

$\mathrm{scFv} \quad$ single-chain variable fragment

SCOK strained cyclooct-2-yne-L-lysine

SD standard deviation

SDS sodium dodecyl sulphate

SDS-PAGE sodium dodecyl sulphate polyacrylamide gel electrophoresis

SEM standard error of the mean

SIM structured illumination microscopy

SIMS $\quad$ secondary ion mass spectrometry

SM protein Sec1/Munc18-like protein

SNAP-23 synaptosome-associated protein of $23 \mathrm{kDa}$

SNAP-25 synaptosome-associated protein of $25 \mathrm{kDa}$

SNAP-29 synaptosome-associated protein of $29 \mathrm{kDa}$

SNARE soluble NSF attachment protein receptor

SPAAC strain-promoted alkyne-azide cycloaddition 
SPIEDAC strain-promoted inverse electron-demand Diels-Alder cycloaddition

SPILL $\quad$ specific protein isotopic and fluorescence labeling

STED stimulated emission depletion

STORM stochastic optical reconstruction microscopy

Stx syntaxin

TCOK trans-cyclooct-2-ene-L-lysine

TIRF(M) total internal reflection fluorescence (microscopy)

TMR transmembrane region

tRNA transfer RNA

tRNA $^{\text {Pyl }} \quad$ suppressor pyrroslysyl tRNA

UAA unnatural amino acid

VAMP2 vesicle-associated membrane protein 2

VAMP4 vesicle-associated membrane protein 4

VHH variable domain of heavy chain of heavy-chain antibodies

Vtila- $\beta \quad$ vesicle transport through interaction with t-SNAREs 1a-beta

WB western blot

WT wild-type

YFP yellow fluorescent protein 


\section{ABSTRACT}

The burgeoning field of genetic code expansion provides new tools for specifically labeling proteins for a variety of applications. Clickable non-canonical amino acids (ncAA) have been refined for almost-quantitative and highly selective reactions with complementary probes.

In my thesis work I have adapted genetic code expansion for two biological questions. First, I used ncAA incorporation and click reaction to test the effect of fluorescent protein (FP) tagging on the nanoscale organization of target proteins. Second, I used these tools to generate a genetically-encoded scheme for specific protein labeling in non-optical nanoscale imaging.

Protein localization and behavior has been regularly tested using FPs. Conventional imaging experiments using FPs are simple and efficient, which renders FPs attractive also for superresolution microscopy (nanoscopy). Nevertheless, FPs have been claimed to induce the nanoscale aggregation of target proteins. Therefore, the effects of FP-tagging on the nanoscale organization behavior of the target proteins needed to be tested, in an unbiased fashion, using a reporter that is smaller and less artifact-prone than the FPs. I relied on the specific incorporation of the ncAA propargyl-L-lysine (PRK) into the FP chimeras of 26 proteins of interest, both cytosolic and membrane attached. The proteins were coupled via click chemistry to fluorescent probes suitable for either stimulated emission depletion microscopy (STED) or ground state depletion followed by individual molecule return (GSDIM). Analysis of the resulting images showed that FP tagging has negligible effects on most proteins, and therefore supported the use of FPs in nanoscale imaging.

Optical microscopy is not the only nanoscale imaging approach that can be used at the moment. Nanoscale secondary mass spectrometry (NanoSIMS) relies on isotope measurements to reach a similar resolution domain. However, it lacks genetically encoded, FP-like tools. For this purpose I developed a novel labeling scheme, for specific protein isotopic and fluorescence labeling (SPILL). It involves the incorporation of ncAA PRK followed by the reaction with two novel probes containing isotopes that are not normally abundant in cells, ${ }^{19} \mathrm{~F}$ and ${ }^{15} \mathrm{~N}$. These isotopic probes can be imaged in SIMS akin to GFP in optical microscopy, and enable the high resolution imaging of many cellular parameters, including protein turnover. 



\section{INTRODUCTION}

\subsection{The Importance of Probe Size in Detecting Proteins}

The probes used for detecting proteins come in all shapes and sizes (see Figure 1-1). In the case of affinity probes (i.e. antibodies, nanobodies, aptamers, etc), their size directly influences the ability of each probe to penetrate the sample and reach the target protein. It is expected that larger probes, such as antibodies, would be less efficient in labeling proteins. This effect has been indeed observed when comparing aptamer stainings with immunostainings. The latter results in a much lower labeling density and fails to reveal important morphologic aspects in the sample compared with the smaller aptamers (Opazo et al., 2012). Aptamers are oligonucleotides (RNA or DNA) selected in vitro to detect specific targets and represent another class of affinity probes (Ellington and Szostak, 1990). Their size is variable and they can be bound to virtually any fluorophore (Opazo et al., 2012).

In super-resolution microscopy techniques, the size of the probe can influence the detection accuracy by introducing a displacement from the protein of interest. This issue is amplified by the use of a primary plus secondary antibody detection system (Opazo et al., 2012; Ries et al., 2012; Szymborska et al., 2013). The resulting complex is $\sim 25 \mathrm{~nm}$ and induces a displacement of $\sim 15 \mathrm{~nm}$ from the target. For instance, if a synaptic vesicle with a diameter of about $40 \mathrm{~nm}$ (Hu et al., 2008; Takamori et al., 2006) is labeled with a primary and secondary antibody complex, with a displacement of about half its size. This clearly demonstrates the requirement for smaller probes, especially for super-resolution microscopy investigations.

As super-resolution microscopy techniques reach nowadays $20-30 \mathrm{~nm}$ on a routine basis, the precision with which probes label their target is an important aspect to be taken into account. Smaller affinity probes derived from immunoglobulin ( $\operatorname{IgG}$ ) molecules (see Figure 1-2) represent a better alternative to conventional antibodies. It has been shown that if antibodies $(\sim 150 \mathrm{kDa}, \sim 13 \mathrm{~nm}$ in size) are replaced with the smaller camelid-derived nanobodies (or VHH, variable domain of heavy chain of heavy-chain antibodies), then the labeling improves considerably, achieving a higher density and a smaller detected spot size (Ries et 
al., 2012). Antibodies have also been shown to induce the clustering of target proteins, especially when applied on live or on insufficiently fixed cells (Tanaka et al., 2010).
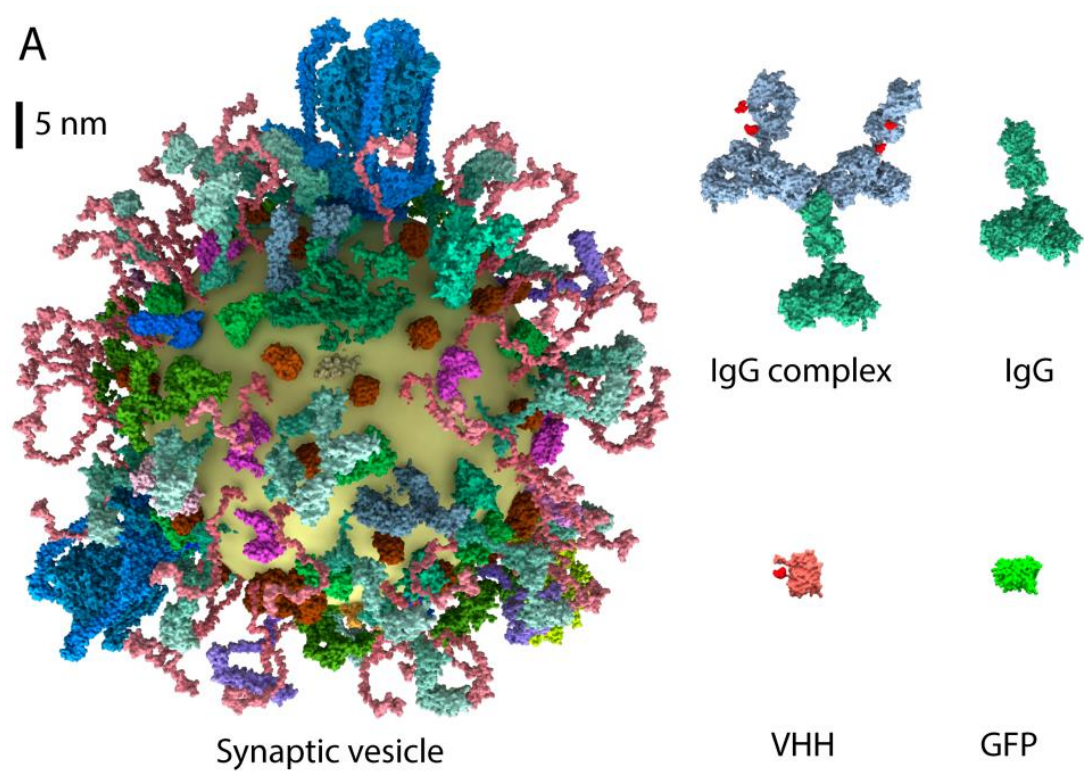

$\lg G$

Fab
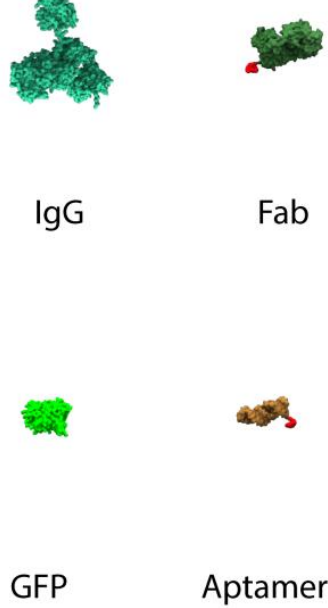

GFP
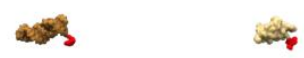

B
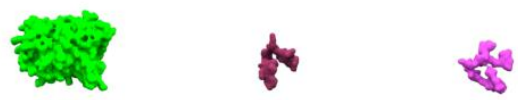

SK155

PRK

Figure 1-1 Size comparison for the different protein affinity probes and tags

A. The structure of a synaptic vesicle is given for comparison with the sizes of the most widely used affinity probes and the genetically-encoded GFP tag. B. The probes and dyes used in the study are shown for direct size comparison with GFP. The 3D renderings of the molecules were generated by Burkhard Rammner. Note that the Atto647N values are given for the carboxy form because the structure of its azide derivative is not disclosed by the producer. In constrast, the Star635P and the Alexa647N values represent the ones for the azide derivatives. Abbreviations: VHH - variable domain of heavy chain of heavy-chain antibodies; $\mathrm{scFv}$ - single-chain variable fragment;. Fab - antigenbinding. Scale bars for $\mathbf{A}$ and $\mathbf{B}$ are $5 \mu \mathrm{m}$ and $1 \mu \mathrm{m}$, respectively. Panel $\mathbf{A}$ was adapted from Fornasiero and Opazo, 2015 with permission. The sizes and molecular weights of the different probes can be found in Table A-1 in the Appendix. 


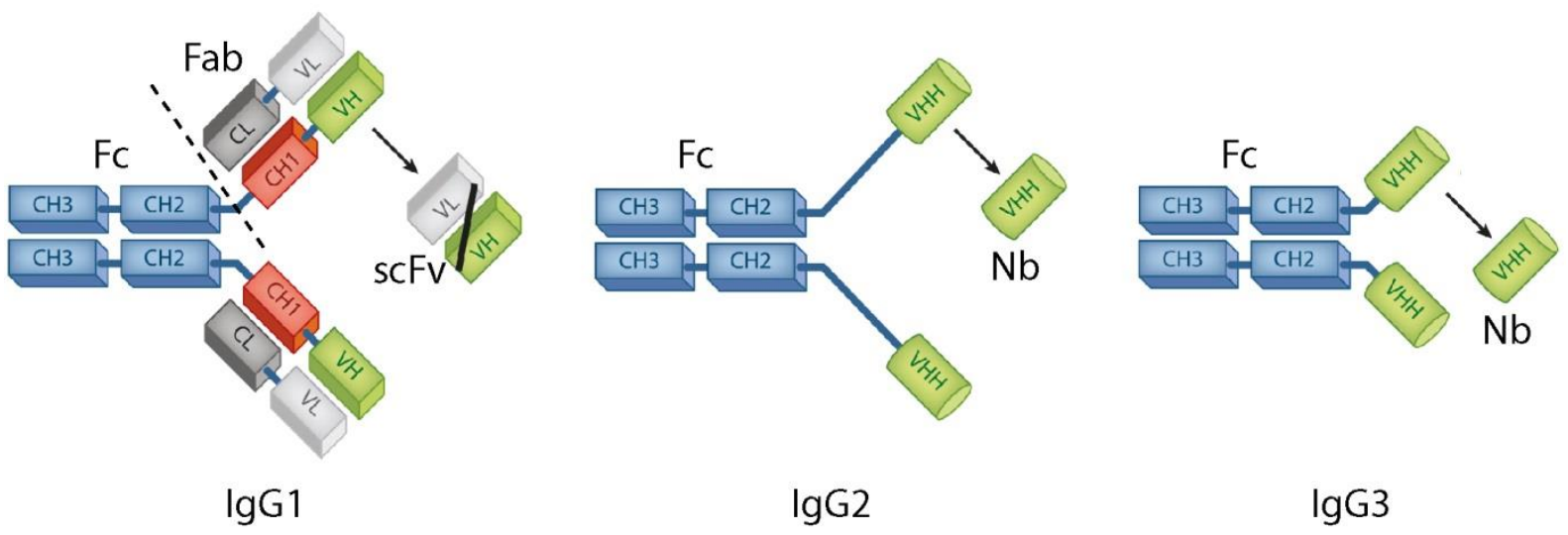

\section{Figure 1-2 Antibodies and derived probes}

Schematic depictions of the three main types of immunoglobulin $\mathrm{G}(\mathrm{IgG})$ molecules. IgG1 antibodies are found in all mammals, while IgG2 and IgG3 are found only in camelids. A convetional antibody molecule contains two light chains with a constant (CL; dark grey) and a variable domain (VL; light grey) and two heavy chains with three constant domains (CH1-3, blue) and one variable domain (VH; green box). In contrast, IgG2 and IgG3 have only two heavy chains, each of them with two constant domains ( $\mathrm{CH} 1$ and $\mathrm{CH} 2$ ) and a variable (VHH; green cylinder). The IgG2 and IgG3 are differentiated based on the size of the linker region between the $\mathrm{CH} 2$ and the VHH domains. For IgG1 the smallest unit that retains antigenicity consists of a fusion between the $\mathrm{VL}$ and the $\mathrm{VH}$ domain using a peptide linker to obtain what is known as a single-chain variable fragment (scFv). Fab (antigen-binding) and Fc (crystallizable) fragments are obtained from IgG1 by papain digestion. For both $\operatorname{IgG} 2$ and $\mathrm{IgG} 3$, the VHH domain, also known as a nanobody, retains the antigen binding properties. Adapted with permission from Muyldermans, 2013.

\subsubsection{Genetically Encoded Tags}

The main class of genetically encoded probes is represented by fluorescent proteins (FPs). Among them, the green fluorescent protein (GFP; Figure 1-3) was identified to be responsible for the green fluorescence of the marine jellyfish Aequorea victoria (Morin and Hastings, 1971; Morise et al., 1974). The wild-type GFP is $27 \mathrm{kDa}$ protein containing 238 amino acids (Prasher et al., 1992). GFP was first employed as a marker for gene expression in bacteria as well as in eukaryotic organisms (Chalfie et al., 1994; Inouye and Tsuji, 1994) and has found major applications as a reporter for protein expression ever since (Phillips, 2001).

The GFP chromophore is an imidazolinone dye generated by oxidation from a hexapeptide which is located in the center of the beta-barrel molecule (Figure 1-3; Ormö et al., 1996; 
Frommer et al., 2009). In the past years, many GFP variants have been produced via mutagenesis that not only fluoresce in different colors but also act as $\mathrm{pH}$ and metal-ion sensors, photoactivatable dyes, etc (Tsien, 1998; Remington, 2006; Shaner et al., 2007) . In spite of all of these improvements brought to the GFP molecule as well as other FPs, their photochemical and photophysical properties are not as good as the ones achieved by synthetic fluorescent dyes, especially for the super-resolution microscopy with powerful lasers (Morozova et al., 2010).

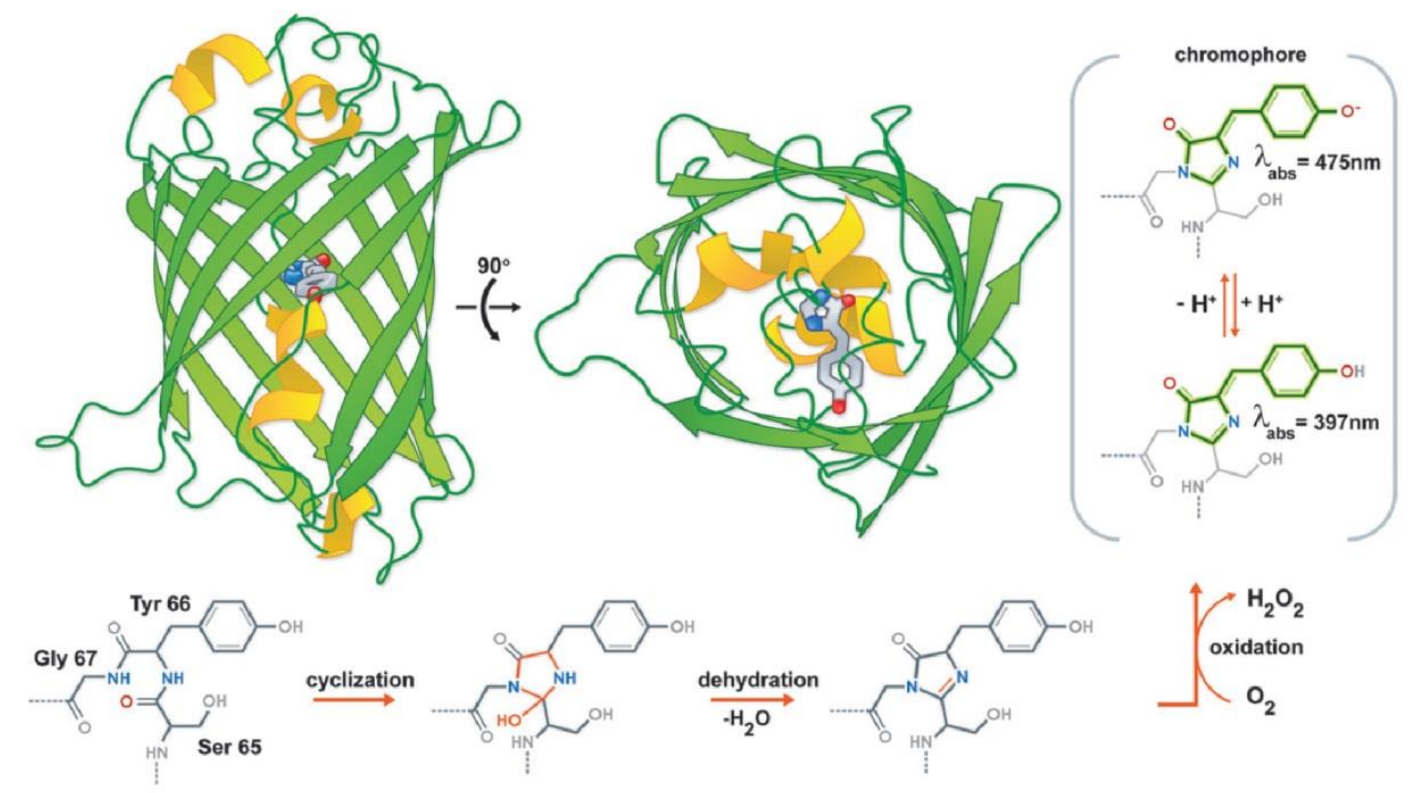

\section{Figure 1-3 The green fluorescent protein (GFP) fluorophore}

The $\beta$-barrel structure of the Aequoria vitoria green fluorescent protein (PDB ID: 1EMA) is shown from two different perspectives. The formations of its intrinsic fluorophore incloves the cyclization of adjacent residues, Ser-65, Tyr-66, and Gly-67, followed by dehydration and oxidation. Reproduced from Frommer et al., 2009, with permission.

Another issue associated with GFP is the fact that it tends to form dimers. Even in the case of engineered versions that were designed to alleviate this problem (e.g. enhanced GFP or EGFP), dimerization has been reported to occur (Jain et al., 2001; Chen et al., 2002). Therefore, FPs might induce the formation of dimers and oligomers when used as protein tags. This issue has become apparently not long ago when visualizing FPs in superresolution microscopy, the only technique capable to discern such artifacts (Annibale et al., 2011a).

Other genetically encoded probes include the smaller tetracysteine (Cys-Cys-X-Y-Cys-Cys, Griffin et al., 1998), CLIP (O-2-benzylcytosine; Gautier et al., 2008), SNAP (engineered version of O-6-methylguanine-DNA methyltransferase; Keppler et al., 2003), and Halo 
(haloalkane dehalogenase, monomeric protein from bacteria; Los et al., 2008) tags. These tags have been shown to label proteins with good yields for live-cell fluorescence microscopy (Adams et al., 2002; Gautier et al., 2009; Wombacher and Cornish, 2011). They are all smaller than FPs but similarly to the latter, they cannot be inserted anywhere in the protein of interest. Moreover, in spite of its small size, the tetracysteine tag has been reported to affect the intracellular localization of the HIV-1 group-specific antigen (Gag) protein depending on the position (Rudner et al., 2005).

Non-canonical amino acids, also referred to as unnatural amino acids, represent the minimal modifications that can be introduced to a peptide chain. For their incorporation into the target proteins, they rely on the expansion of the genetic code. Their versatility and possible pitfalls are discussed in the next chapter.

\subsection{Genetic Code Expansion and Its Applications}

The canonical version of the genetic code contains a total of 64 possible triplet codons. Out of all these possible triplet combinations, 61 are sense codons and encode for a total of 20 canonical amino acids, whereas the remaining three nonsense (or stop) codons (UAA Ochre, UAG - Amber, and UGA - Opal) are recognized during translation by release factors (RF-1 and RF-2 for prokaryotes and eRF-1 for eukaryotes; Nakamura et al., 1996).

The genetic code was initially considered to be "frozen" in its canonical form comprising 20 amino acids (Crick, 1968; Thomas, 1970) because of its universality in all organisms known at that time. In recent years, many differences observed in the genetic code of various organisms suggest that it is not only flexible but also that it is evolving together with the current organisms. Small deviations from this canon were discovered, first in mitochondria and then also in the genomes of organisms like Mycoplasma sp., green algae, and Candida $s p$. (Osawa et al., 1992). Selenocysteine and pyrrolysine are now considered the $21^{\text {st }}$ and $22^{\text {nd }}$ amino acids in the canonical set (Ambrogelly et al., 2007). They are incorporated in response to Opal (UGA) and Amber (UAG) stop codons (Söll, 1988; Srinivasan et al., 2002).

The genetic code has a variable degree of degeneracy, with amino acids being encoded by one up to six different codons. The translation termination signal is also degenerate having three different codons that are recognized by release factors. This feature of the genetic code can be exploited to reassign the least used codons for the encoding of a non-canonical amino acids. This strategy has been optimized especially for the suppression of the Amber stop 
codon, both in vitro and in vivo for both prokaryotic and eukaryotic cells (Noren et al., 1989, 1990; Furter, 1998; Liu et al., 2007).

Non-canonical amino acids can be incorporated by using their structural similarity to canonical amino acids (e.g. L-azidohomoalanine mimics methionine) and are thus used as general protein labels (Johnson et al., 2010). Another way to label proteins with ncAAs is to site-specifically codify them into a protein of interest using a dedicated bioorthogonal machinery (Liu and Schultz, 2010), which implies the lack of cross-reactivity with the endogenous components.

\subsubsection{General Protein Labeling}

For general protein labeling, homologs of the canonical amino acids are employed. These are similar enough to be recognized by the same synthetase(s) and used for the aminoacylation of the corresponding tRNA(s). For proper labeling to take place, only essential amino acids can be substituted because the rest of the amino acids are produced by the cells and would therefore outcompete the non-canonical amino acid.

Selenocysteine is among the first ncAAs that have been introduced into proteins for phase determination in crystallography (Cowie and Cohen, 1957). More recently, clickable noncanonical analogues of the methionine azido-homoalanine (AHA) and homopropargylglycine (HPG) were implementated for proteome-wide labeling assays (Link et al., 2006). These ncAAs have also been used to label all proteins either for pulse-chase experiments (Dieterich et al., 2007, 2010) or as tools to investigate the general structure of proteins in membranes (Saka et al., 2014a).

This technique requires only the addition of the ncAA to the medium of the cells that lacks its endogenous analog. But it cannot be used to label specific proteins without the help of larger probes such as antibodies or fluorescent proteins tags.

\subsubsection{Specific Protein Labeling}

Tagging specific proteins with genetic precision is preferable to the general protein labeling approach.

Genetic code expansion involves adding new (non-canonical) amino acids to the repertoire present in the cells. Several requirements need to be fulfilledfor this technique to specifically label a protein of interest (Figure 1-4). First, a pair consisting of a suppressor aminoacyl- 
tRNA synthetase and a tRNA (RS/tRNA) have to be introduced (via transfection) in the cell. These heterologous pair has to be bioorthogonal, meaning that it should not cross-react with the machinery present in the cell. Second, a new codon (i.e. nonsense or four-base codons) has to be assigned (or re-assigned) for the incorporation of a ncAA. Last but not least, the ncAA that has to be efficiently uptaken into the cells and recognized only by the orthogonal RS/tRNA pair.

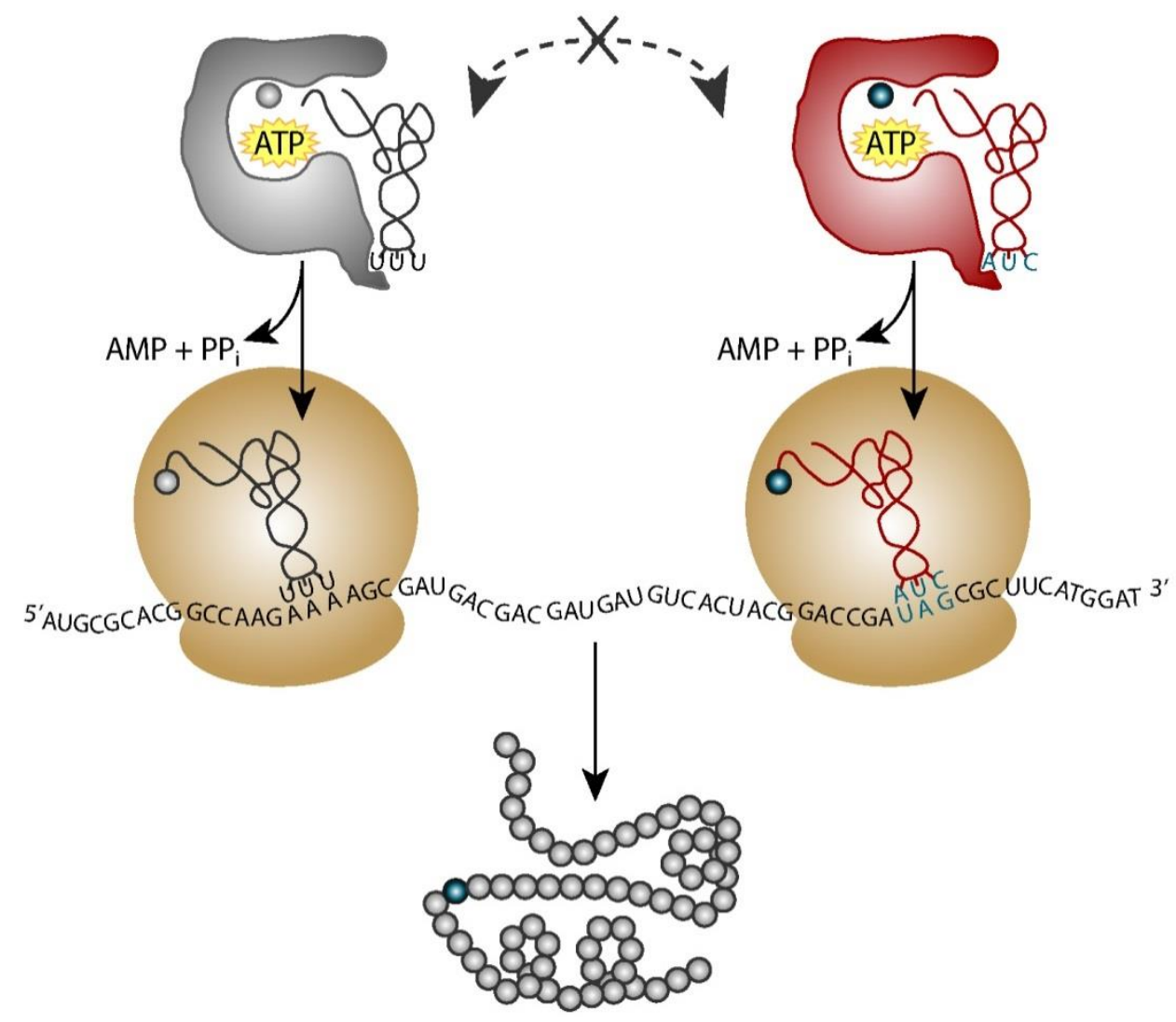

Figure 1-4 The principle of ncAA incorporation

The genetic encoding or incorporation of non-canonical amino acids (ncAAs) involves expressing a bioorthogonal synthetase-tRNA couple (RS/tRNA; shown in red) and a mutagenized protein of interest that accommodates an Amber stop codon in its coding sequence. At the same time, the ncAA should be provided to the cell medium. The synthetase specifically aminoacylates the suppressor tRNA with the ncAA in the presence of ATP (in yellow). The endogenous amino acids, tRNA and sythetase are depicted in grey shades, while the ncAA, the Amber stop and the anticodon region of the suppressor tRNA are shown in blue-green. During translation of the protein of interest, the anticodon region of the suppressor tRNA will recognize the Amber stop codon on the mRNA. Then the ribosome (shown in light brown) will direct the incorporation of the ncAA into the primary sequence of the protein of interest. 
The easiest way to provide a host cell with a new RS/tRNA pair is to use one from a different organism that does not cross-aminoacylate components of the target cell. Many candidate pairs have been described in literature: the glutaminyl-tRNA/synthetase from $S$. cerevisiae that tolerates a wide range of ncAAs for incorporation (Liu and Schultz, 1999), the tyrosyl RS/tRNA from Methanococcus jannaschii (Wang et al., 2000) required mutations in the tRNA to reduce aminoacylation by host synthetases (Wang et al., 2001), and the pyrrolysyl RS/tRNA from Methanosarcina barkeri or mazei (Srinivasan et al., 2002; Blight et al., 2004). The pyrrolysyl RS/tRNA pair was demonstrated to be compatible with a wide range of hosts from $E$. coli to mammalian cells and is at the moment one of the most widely used (Polycarpo et al., 2006; Neumann et al., 2008; Chen et al., 2009).

So far approximately 100 ncAAs (Liu and Schultz, 2010; Li and Liu, 2014) have been described for different applications and incorporated into proteins expressed in bacteria (Wang et al., 2001), yeast (Chin, 2003), mammalian cells (Sakamoto et al., 2002; Liu et al., 2007) as well as animals (Greiss and Chin, 2011; Bianco et al., 2012; Parrish et al., 2012).

Limitations involve the permeability of the ncAA or the propensity with which it is uptaken into cells, as well as the ribosome requirements and its compatibility with synthetase active site (i.e. there is a limited ability to mutagenize the catalytic pocket of the sythetase).

With appropriate RS/tRNA pairs, ncAAs offer broad possibilities to vary the structural, chemical and spectroscopic properties of the proteins they tag. Applications of genetic code expansion include new tools for investigating protein function on a cellular level and also generation of proteins with enhanced functionality and/or properties.

\subsection{Labeling Non-Canonical Amino Acids via Click Chemistry}

Non-canonical amino acids require not only to be specifically incorporated in the proteins of interest, but also to be specifically coupled to probes for later detection.

Barry Sharpless coined the term "click chemistry" to denote a highly efficient and selective reaction between relatively small and interchangeable components (Kolb et al., 2001). Click chemistry encompasses two broad classes of reactions: cycloadditions (Alder and Stein, 1931, 1933; Huisgen, 1963; Boger, 1986) and the Staudinger ligation (Staudinger and Meyer, 1919). The latter will not be discussed here because it is less efficient and thereby has fewer biological applications than the cycloaddition reactions (Lang and Chin, 2014). 
Therefore, throughout this work, I use click chemistry when referring to Huisgen or inverseelectron demand Diels-Alder cycloaddition.

Huisgen [3 + 2] cycloadditions involve the reaction of a dipolarophile (e.g. an alkyne or an alkene group) with a 1,3-dipolar compound (e.g. an azide group), and results in the formation of a 5-membered heterocycle (Figure 1-5 and Figure 1-6). Another reaction more recently employed for click chemistry is the inverse-electron demand Diels-Alder [4+2] cycloaddition (Figure 1-7). The usual Diels-Alder reaction involves a pericyclic reaction between an electron-poor dienophile (alkene) and an electron-rich diene. In the reaction between an alkyne or an alkene and a tetrazine group, the electron demand is reversed.

\subsubsection{Copper-Catalyzed Alkyne-Azide Cycloaddition (CuAAC)}

The copper-catalyzed alkyne-azide cycloaddition, in short CuAAC, was introduced in 2002 by Barry Sharpless and Morten Meldal in response to the new concept of bioorthogonal chemistry (Rostovtsev et al., 2002; Meldal and Tomøe, 2008). The latter advances the idea that probes for labeling reactions should react specifically and in high yield with their click partners under physiological conditions without interfering with other biomolecules present in cells. In addition, the bioorthogonal reactants should be not only kinetically and thermodynamically, but also metabolically stable (Lang and Chin, 2014).

Azides and alkynes are not normally found in cells (Boyce and Bertozzi, 2011). They react specifically and in high yield in the presence of copper (I) to form stable triazoles, which act as covalent links between the protein of interest and the probe (Figure 1-5). This makes them good candidates for labeling proteins. However, the requirement for $\mathrm{Cu}(\mathrm{I})$ as a catalyzer raises problems in terms of toxicity for the cells if live labeling is attempted (Wolbers et al., 2006; Hong et al., 2011).

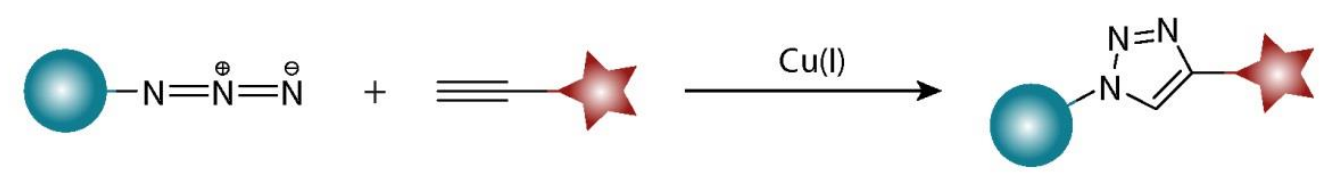

Figure 1-5 The copper-catalyzed alkyne-azide cycloaddition (CuAAC)

Scheme depicting the cycloaddition of a terminal alkyne to an azide in the presence of copper(I). The blue sphere and the red star indicate the protein of interest and fluorescent probe, respectively. The azide and the alkyne groups are, in principle, interchangeably located either on the protein of interest or on the dye molecule. 


\subsubsection{Strain-Promoted Alkyne-Azide Cycloaddition (SPAAC)}

The reaction of strained alkynes with azides (see Figure 1-6), also known as SPAAC, does not require a metal catalyzer (Alder and Stein, 1931, 1933; Wittig and Krebs, 1961; Agard et al., 2004) and has been proposed as a substitute for CuAAC (Plass et al., 2011). But in the absence of any catalyzer, the strain on the alkyne group is not sufficient to provide high enough rates of reaction. For this reaction to take place, it is necessary to use high concentrations of fluorescent dye when labeling the samples in order to push the equilibrium towards the cycloaddition products (Plass et al., 2011; Lang and Chin, 2014).

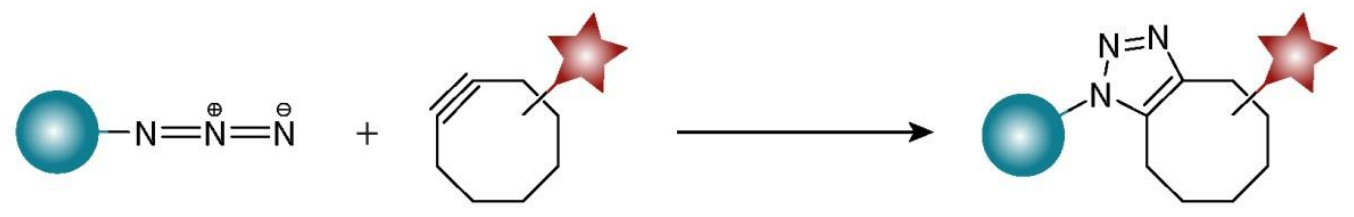

Figure 1-6 The strain-promoted alkyne-azide cycloaddition (SPAAC)

This reaction relies on the relatively high reactivity of the alkyne group, which is due to the strain imposed by the eight (or nine) member ring on the alkyne group. The sphere and the star represent interchangeably the protein of interest or the fluorescent probe.

\subsubsection{Strain-Promoted Inverse-Electron Demand Diels-Alder Cycloaddition (SPIEDAC)}

The SPIEDAC reaction (Nikić et al., 2014) involves the cycloaddition of a strained alkene or alkyne to a tetrazine heterocycle (Figure 1-7) with the release of nitrogen gas (Boger, 1986; Blackman et al., 2008). For alkenes this reaction results in the formation of a dihydropyridazine, while pyridazines are generated for alkynes. This reaction exceeds in speed the CuAAC and is a good candidate for in vivo studies (Plass et al., 2012; Nikić et al., 2015; Uttamapinant et al., 2015). Interestingly, the tetrazine group has yet another application, namely that it makes fluorogenic probes out of some of the fluorophores it is attached to (Devaraj et al., 2010; Lang et al., 2012). These dyes exhibit quenched fluorescence, but upon reaction with alkyne or alkenes the fluorescence is restored. 


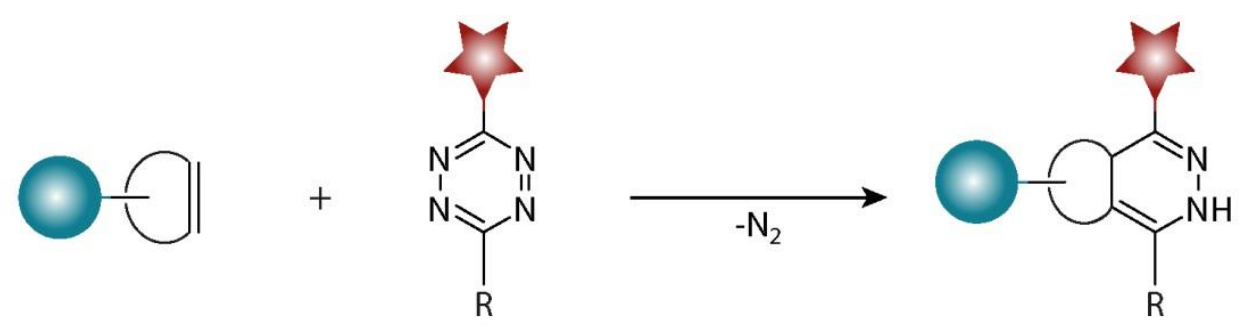

\section{Figure 1-7 The strain-promoted inverse-electron demand Diels-Alder cycloaddition (SPIEDAC)}

This reaction involves the cycloaddition of a strained alkene (or alkyne) to a tetrazine group to generate a dihydropyridazine (or pyridazine) link between the protein of interest (in blue) and the fluorescent probe (in red). This reaction is made irreversible by the release of nitrogen gas. $\mathrm{R}$ denotes a substituent (e.g. $\mathrm{CH}_{3}$ or $\mathrm{H}$, among others), which can modulate the reactivity of the tetrazine group in SPIEDAC.

\subsection{A Technical Perspective on Super-Resolution Microscopy}

The advent of fluorescent labeling has brought about a rapid increase in the applications the fluorescent probes have in disciplines that are related to biology. Nowadays, fluorescence microscopy is an established tool to investigate biological materials (Stephens and Allan, 2003; Lichtman and Conchello, 2005).

The particular strength of fluorescence microscopy lies in the existence of specific probes and its compatibility with live cell imaging. Thus fluorescence microscopy enables reliable visualization of one or multiple colors with little background.

The majority of the biological molecules have very low intrinsic fluorescent properties and, in order to be detected, probes have to be attached to them to enable their visualization. Compared to normal stains, fluorescent labels offer far better contrast and enabled the improvements in light microscopy.

Quinine, an anti-malarial compound, played an important role in the discovery of fluorescence and is therefore considered the first small-molecule fluorophore. Herschel was the pioneer who noticed the emission generated by the otherwise colorless solution of quinine in direct sunlight (Herschel, 1843). George Gabriel Stokes coined the term "fluorescence" based on the observation that if a quinine solution is illuminated with blue light (short wavelength) the color of the light returning from the solution is green-yellow (longer wavelength; Stokes, 1852). The difference in the absorption and emission maxima is termed in his honor "Stokes shift" (see Figure 1-8 A). 
The fluorescence phenomenon is illustrated by the simplified Jablonski diagram from Figure 1-8 B (Lichtman and Conchello, 2005). It involves the absorption of light of a particular wavelength (or energy). This raises the molecule from the ground state into an excited state. The difference in energy between the ground state and the excited state is equal to the absorbed energy. However some of it is dissipated (i.e. through internal conversion, vibrational relaxation or interactions with the solvent). As a result, the wavelength of the light emitted when the molecule falls back into the ground state is higher (i.e. lower energy). The decay to the ground state can also occur in a non-radiative fashion, also known as quenching (Lavis and Raines, 2008).

A

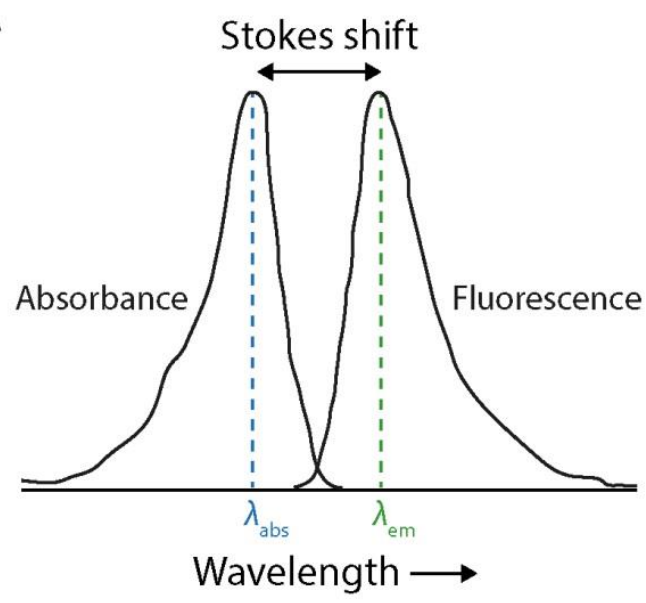

B Jablonski diagram

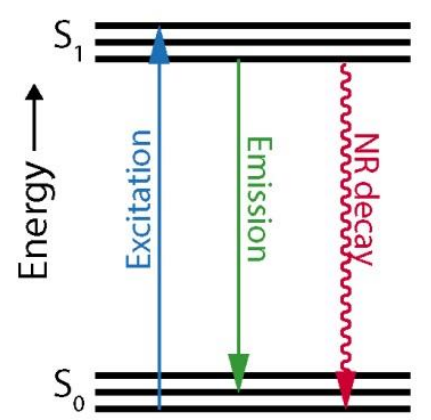

Figure 1-8 Absorption and emission of fluorescent dyes

A. An absorption-emission spectrum for a fluorescent dye with a large Stokes shift. The absorption and emission maxima are annotated $\lambda_{\mathrm{abs}}$ and $\lambda_{\mathrm{em}}$, respectively. B. A simplified Jablonski diagram representing the absorption of a photon by a fluorescent dye molecule and the promotion of the molecule from the ground state $\left(\mathrm{S}_{0}\right)$ to an excited state $\left(\mathrm{S}_{1}\right)$, a phenomenon also known as excitation (in blue). The dye molecule can return to the ground state by release this energy either through a radiative process (fluorescence; in green) or through a non-radiative (NR) decay or quenching (in red). Note that the emitted light is of a longer wavelength (lower energy) due to processes, such as internal conversion and vibrational relaxation, not shown here for clarity purposes.

The Stokes shift is an important parameter that describes the tendency of a fluorophore to undergo self-quenching, namely the bigger the Stokes shift, the less prone the dye is to selfquench. Other important properties include: the quantum yield (QY) describes the ratio between the emitted photons versus the absorbed ones; the photostability is determined by the fluorophore structure and inversely scales with photobleaching reactions; the lifetime of the excited state (typically 0.1-100 ns) is important for time-resolved imaging (Bright and Munson, 2003; Lavis and Raines, 2008, 2014). Even though the fluorescence phenomenon is not restricted to the visible range of the electromagnetic spectrum, fluorescence microscopy 
employs with few exceptions (for the infrared and the ultraviolet spectrum) mostly this range.

Single fluorophores present in the sample are blurred by the optical system of the microscope to dots of $\sim 200 \mathrm{~nm}$ and $\sim 700 \mathrm{~nm}$ in the in the lateral and axial direction, respectively. This blurring is due to the diffraction of light and is described by a point spread function (PSF), which is a characteristic of the microscope. The maximum resolution attained in conventional microscopy techniques (see Equation 1) is given by the properties of the light beam used, namely the wavelength $(\lambda)$, and the configuration of the objective used for imaging, given by the numerical aperture (NA). The mathematical relationship that describes the effect of the wavelength and of the numerical aperture on the resolution is given by Abbe's law (Abbe, 1873, 1883). The full-width at half-maximum (FWHM) of the PSF is often used in microscopy as a measure for the lateral resolution $\left(d_{x, y}\right)$.

$$
d_{x, y}=\frac{\lambda}{2 \mathrm{NA}}
$$

\section{Equation 1. Abbe's formula for the resolution attained in the xy plane}

The lateral resolution $\left(d_{x, y}\right)$ is the minimum distance between two objects that can be distinguished using a lens-based microscope and is given by the ratio of the wavelength $(\lambda)$ to two-fold the numerical aperture (NA).

The numerical aperture as introduced by Ernst Abbe (Abbe, 1881) represents a dimensionless number, which scales with the amount of light collected by the imaging system. It is proportional to the refractive index $(n)$ of the medium in which the lens is employed (e.g. typically in air, water or immersion oil: $n_{\text {air }}=1.00, n_{\text {water }}=1.33$, and $n_{\text {oil }}=1.52$ ) and to the sine of the half-angle $(\theta)$ of the cone of light exiting (or entering) the objective. In practice the upper limit of $\theta$ is about $70^{\circ}$, resulting in a maximum NA of 1.45 for oil immersion objectives.

$$
\mathrm{NA}=n \sin \theta
$$

\section{Equation 2. $\quad$ Numerical aperture (NA) formula}

$n$ is the index of refraction for the medium the objective is placed in, while $\theta$ is the half-angle of the cone of light that can pass through the objective.

In view of the above formulae the best resolution is attained for shorter wavelengths (blue rather than red light) and for objectives with wider numerical apertures (e.g. the oil 
immersed ones). As a result, the highest lateral resolution achievable for a microscope using an oil immersion objective and detecting blue-light fluorescence $(\lambda \sim 400 \mathrm{~nm})$ is $\sim 200 \mathrm{~nm}$. Therefore, using this technique cellular elements or protein assemblies closer than $200 \mathrm{~nm}$ appear as one blurred object, hindering their accurate localization and investigation.

For more than a century, Abbe's law has been considered a major deterrent in going beyond the resolution limit. However, in the last decades various techniques have been developed to overcome the resolution limit. Super-resolution imaging can be achieved either by structured illumination microscopy (SIM; Gustafsson, 2000) or functional techniques that employ photophysical or photochemical phenomena to achieve sub-diffraction imaging. The latter category of has gained a widespread popularity in the last decade and includes ensemble methods, such as stimulated emission depletion (STED) microscopy, as well as single molecule approaches such as ground-state depletion followed by individual molecule return (GSDIM), photoactivation localization microscopy (PALM), and stochastic reconstruction microscopy (STORM). Progresses in these techniques in the last two decades have spearheaded a major paradigm change in the focus of biology towards the nanoscale (Hell, 2007; Huang, 2010; Galbraith and Galbraith, 2011; van de Linde et al., 2012).

\subsubsection{Ensemble Methods: STED Nanoscopy}

STED achieves sub-diffraction imaging using stimulated emission to quench the fluorophores on the margins of the fluorescent spot thereby constricting the size of its PSF (Hell and Wichmann, 1994, see Figure 1-9 A). In practice this is done by scanning the sample using a STED beam with a zero intensity at its center ("OFF" laser) overimposed on the excitation beam ("ON" laser; see Figure 1-9 B) and it results in a fluorescent spot smaller than the diffraction limit $(<200 \mathrm{~nm})$. The STED effect depends on the intensity of the depletion laser and the requirements of the fluorescent dye to undergo stimulated emission (Figure 1-9 C). This in turn influences the attained resolution. As shown by Westphal and Hell, 2005, the lateral resolution $\left(d_{x y}\right)$ of the STED microscope is given by a modified form of Abbe's formula (compare Equation 3 with Equation 1).

$$
d_{x, y} \approx \frac{\lambda}{\mathrm{NA} \sqrt{1+\frac{I}{I_{s}}}}
$$

Equation 3. Resolution formula for STED microscopy 
The lateral resolution $\left(d_{x, y}\right)$ the STED microscope is a modified form of Abbe's formula (Westphal and Hell, 2005), where $\lambda$ is the wavelength, NA is the the numerical aperture, while $I$ and $I_{S}$ represent the intensities of the STED laser and of the saturation value for turning off a specific dye.

A

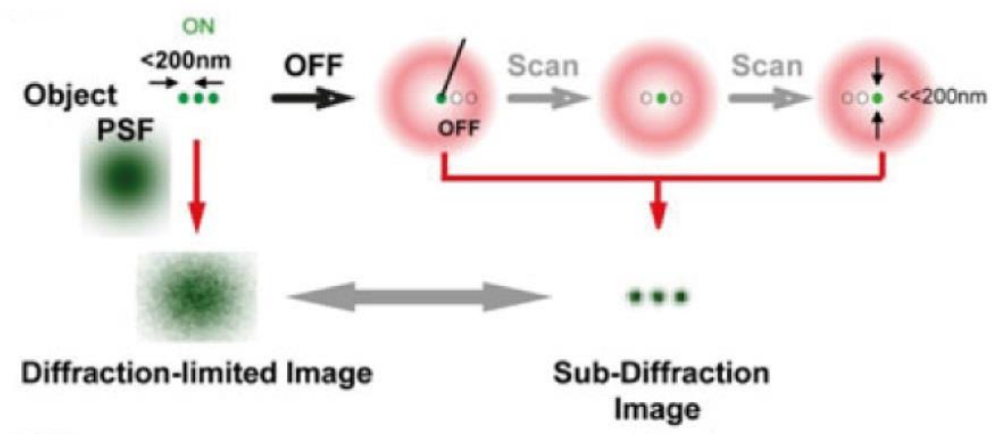

B

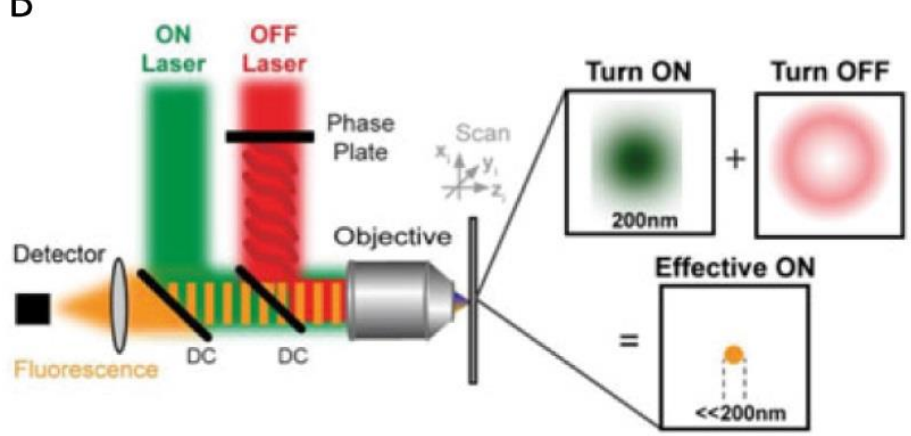

C

Figure 1-9

The STED nanoscopy principle

A. Comparison between the point spread function in confocal microscopy (in green, on the left) and the effective point spread function in STED microscopy (green central dot surrounded by the redshifted STED beam; on the right). The spots detected in STED are smaller than the diffraction limit because the fluorophores on the margin of the diffraction limited spot are not allowed to fluoresce. $\mathbf{B}$. The STED microscope is built on the principles of a confocal setup in which a laser ("ON" laser; in green) is used to excite the fluorophores present in the sample. For stimulated emission, an infrared laser ("OFF" laser; in red) is modulated by a vortex plate into a toroid shape (or doughnut) and directed onto the sample where it selectively turns off the fluorophores located on the margins of the excitation beamspot. This results in the confinement of the fluorescence emission to the central region, thereby producing a sub-diffraction spot. C. The STED effect and implicitly the resolution is dependent on the ratio between the "OFF" laser intensity $(I)$ to the saturation intensity $\left(I_{s}\right)$. The latter is a characteristic value of the fluorophore at which fluorescence excitation is halved. Panels A-C are adapted from Eggeling et al., 2015, with permission.

Theoretically, if $\frac{I}{I_{S}} \rightarrow \infty$, then the lateral resolution should no longer be limited by the wavelength of the fluorescent beam and should go beyond molecular or atomic level (Hell, 2003; Westphal and Hell, 2005). However, in practice, this is hard to achieve because the available fluorophores cannot withstand such high laser intensities without bleaching and, more importantly, due to photodamage to the samples. 
STED can achieve up to $30 \mathrm{~nm}$ lateral resolution and at the same time visualize up to three distinct colors (Meyer et al., 2008; Bückers et al., 2011; Göttfert et al., 2013). STED has been used to investigate the cluster structure for proteins involved in exo- and endocytosis (Sieber et al., 2007; Halemani et al., 2010; Hoopmann et al., 2010; Opazo et al., 2010). To continue with, in the STED nanoscopy has been successfully used for the investigation of synaptic vesicles under live imaging conditions (Westphal et al., 2008). The applicability of this technique for live imaging is even more enhanced by using parallelized excitation and detection of the fluorescence (Eggeling et al., 2015).

\subsubsection{Single Molecule Methods: GSDIM, STORM, PALM}

In single molecule localization microscopy, super-resolution is achieved by stochastically switching fluorophores between fluorescent "ON" and non-fluorescent "OFF" states (Figure 1-10 A). In order to precisely localize the signal of each fluorophore, only a sparse subset of fluorophores are in the "ON" state at any given time. Thus it s very unlikely to find two neighbouring fluorophores with overlapping PSF in the "ON" state. Repeating this process thousands of times enables reconstruction of an image with a resolution below the diffraction barrier (see Figure 1-10 D).

A
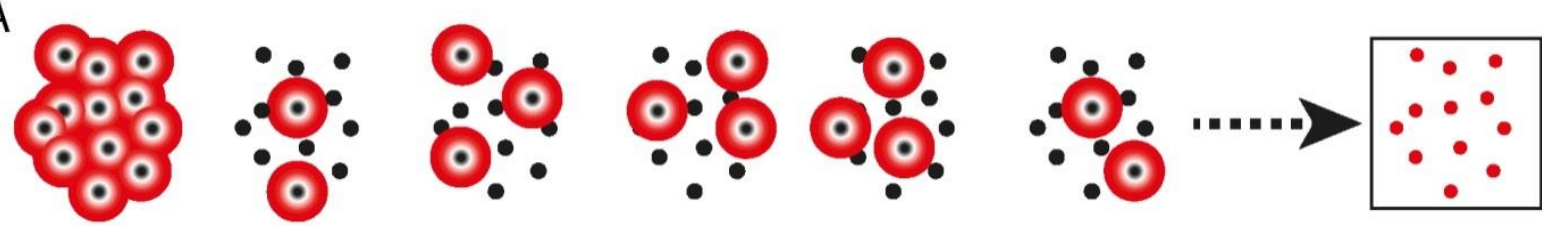

B
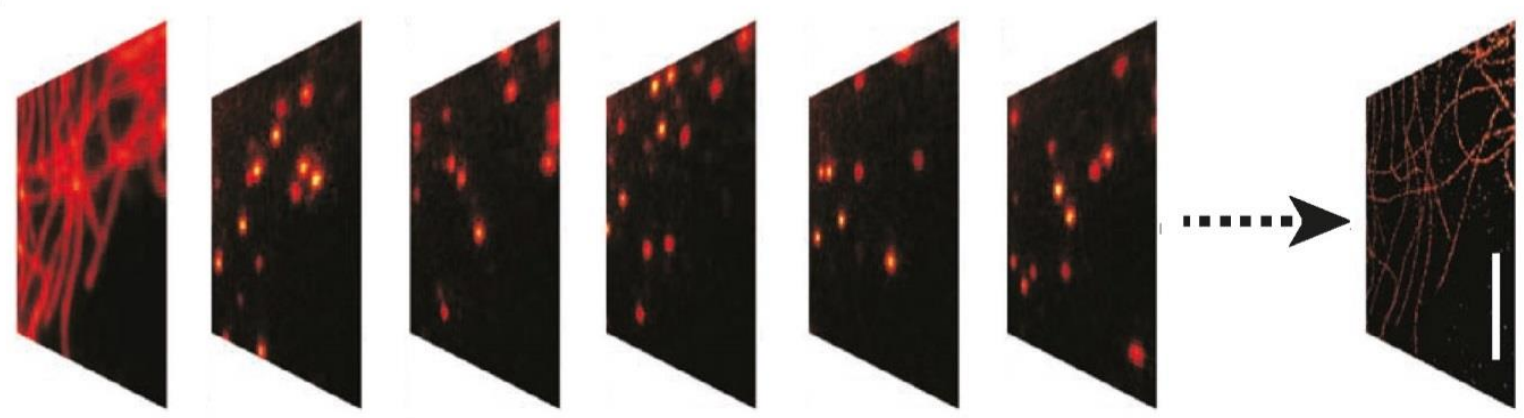

Figure 1-10 The principle behind super-resolution localization microscopy 


\section{Figure 1-10 The principle behind super-resolution localization microscopy}

Localization nanoscopy techniques rely on the sequential activation and time-resolved localization of fluorophores in the sample. A. Scheme depicting how the fluorophores in the samples would look under normal illumination conditions (on the left) or when only a sparse population of fluorophores is activated (the rest of the cases). The fluorophores depicted as black spots are in a dark state ("OFF") and are thus not detected. Fluorophores in the sample are sequentially turned "ON" and "OFF" until a good sample coverage is achieved. The same process is iterated many times to obtain the coordinates for most of the fluorphores in the sample and these coordinates can be used to generate a superresolution image (rectangle on the right). B. Corresponding images for the scheme in A. The diffraction-limited image on the left shows labeled microtubules. In the middle, the sequential detection of single molecules is shown. The centers of their PSFs are calculated and used to produce a sub-diffraction image. Scale bar, $2 \mu \mathrm{m}$. The images in $\mathbf{B}$ were reproduced with permission from van de Linde et al., 2011.

The fluorescence of the single molecules is detected with a sensitive charge-coupled device camera assembled on a conventional widefield fluorescence microscope. Most of the pontilistic setups are in fact total internal reflection fluorescence (TIRF) microscopes. This type of microscope excites fluorophores close to the sample surface with an evanescent wave generated by the total internal reflection of the light illuminating the sample at a certain angle (Axelrod, 1981).

Therefore, pointillistic techniques do not require sophisticated microscopes, but rely on the density and quality of the fluorophores to achieve super-resolution. Fluorescent spots have to be farther apart than the resolution limit (i.e. $>200 \mathrm{~nm}$ ) to enable the correct fitting and to precisely determine the fluorophore position in the lateral plane. The localization accuracy depends on the standard deviation of the PSF and the number of collected photons $(N)$ (see Equation 4; Thompson et al., 2002). A good localization accuracy is the most important prerequisite to achieve super-resolution, however the achievable structural resolution can be impaired by a low labeling density or a large size of the probe.

$$
\sigma_{x, y}=\frac{\sigma}{\sqrt{N}}
$$

\section{Equation 4. Localization accuracy formula for pointillistic nanoscopy methods}

The localization accuracy $\left(\sigma_{x, y}\right)$ scales with the standard deviation of the point spread function $(\sigma)$ and the inverse square root of the number of photons detected $(N)$.

Several pontilistic super-resolution methods have been implemented that differ mainly with respect to the fluorophores they employ. STORM, for example, uses synthetic fluorophores pairs attached to antibodies (Rust et al., 2006; Huang et al., 2008), while PALM relies on photoactivatable fluorescent proteins (Betzig et al., 2006; Hess et al., 2006). 
In contrast to STORM, only a single conventional fluorophore is required for direct STORM (dSTORM; Heilemann et al., 2008), ground-state depletion followed by individual molecule return (GSDIM; Fölling et al., 2008) or reversible photobleaching microscopy (Baddeley et al., 2009). These three techniques are now referred to as either dSTORM or GSDIM, but throughout this study, I employed the GSDIM acronym for the measuremnts I performed.

All the pointillistic super-resolution microscopy techniques generate large data sets and require processing for the final super-resolution image to be obtained. They reach a lateral resolution of $\sim 20 \mathrm{~nm}$ and are amenable to multicolor imaging in both live and fixed cells (Bates et al., 2007; Bückers et al., 2011; Testa et al., 2010). Recent development for the pontilistic techniques include the combination of STORM with astigmatism imaging, which led to the visualization in $3 \mathrm{D}$ of actin microfilaments with a resolution of $10 \mathrm{~nm}$ in the lateral plane and an axial resolution of $20 \mathrm{~nm}$.

\subsection{A Technical Perspective on Secondary Ion Mass Spectrometry (SIMS)}

Secondary ion mass spectrometry (SIMS), also known as ion microprobing, has been first established as an analytical technique for the investigation of the isotopic, elemental and molecular composition of materials (Benninghoven et al., 1987). Nanoscale SIMS, in short NanoSIMS, was designed to achieve high resolution measurements: $50-100 \mathrm{~nm}$ in the lateral direction and up to a few atomic layers in depth (Lechene et al., 2006; Hoppe et al., 2013). This technique has gained over the last decade various applications ranging from biology and ecology to cosmochemistry, geology and soil science (Lechene et al., 2006; Wagner, 2009; Hoppe et al., 2013).

The Cameca NanoSIMS 50L instrument (Cameca) analyzes the colocalization of up to 7 different isotopic species in one run. The NanoSIMS achieves this by hitting the sample with a finely focused primary ion beam which releases atoms, molecular fragments, and, to a smaller extent, ions from the surface. The ions that are formed are concentrated into a secondary ion beam, separated by mass and charge in a magnet, and counted by seven different detectors (one fixed and the other six adjustable; see Figure 1-11). This scanning procedure results in isotope and/or element distribution maps. 


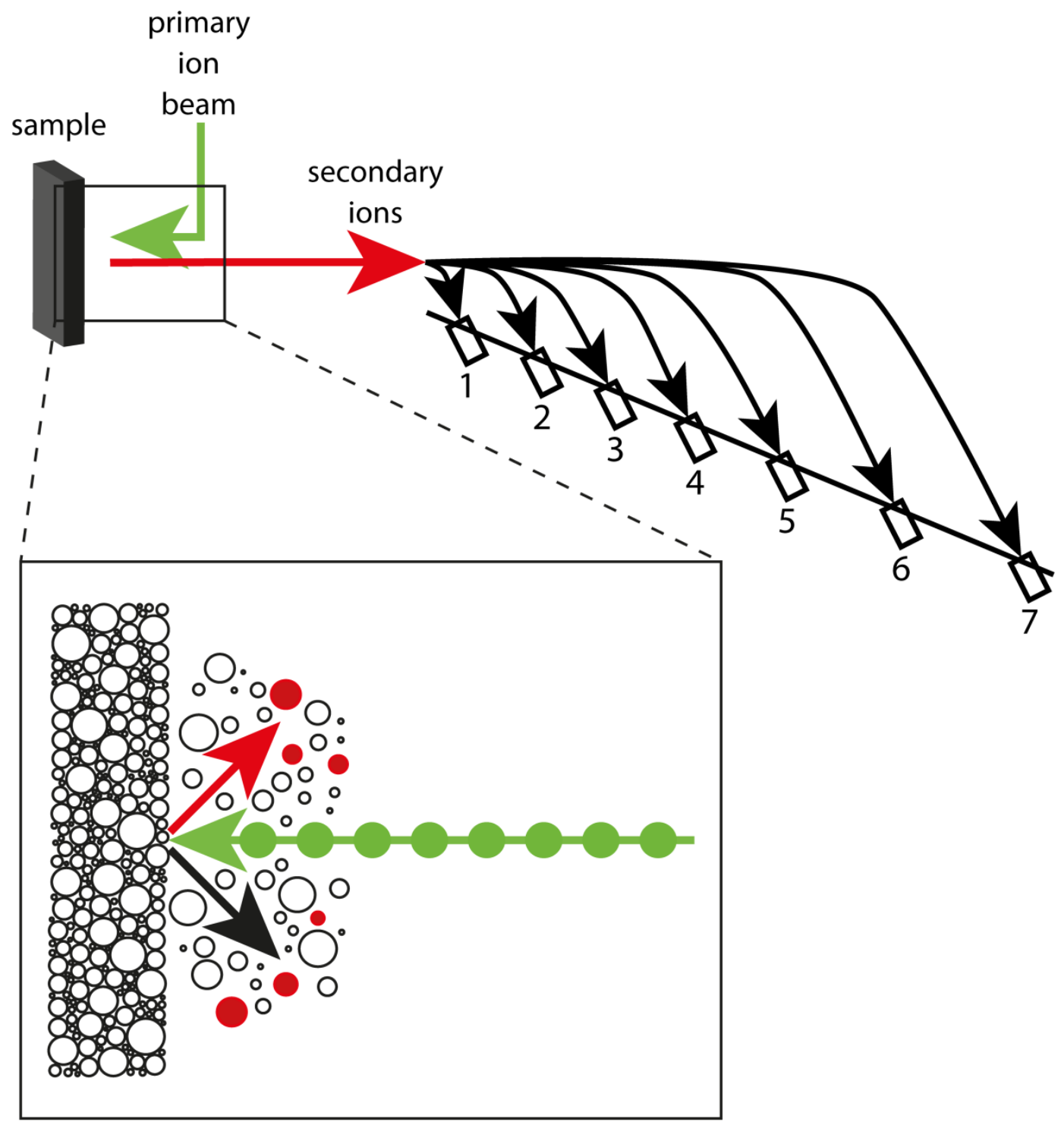

\section{Figure 1-11 The NanoSIMS setup and principle}

In a NanoSIMS instrument a primary ion beam (in green) is finely focused as a small beam spot onto the sample. Here, the primary ions trigger the release of oppositely charged ions and, to a larger extent, uncharged particles (atoms and molecular fragments) in a process called sputtering (see inset below). The secondary ions released from the sample are then collected and form the secondary ion beam (in red). The secondary ions will be separated by mass and charge and detected in parallel by seven detectors (1-7). The NanoSIMS scheme and the inset are adapted from Lechene et al., 2006.

The lateral resolution depends on the primary ion source. $\mathrm{O}^{-}$currents reach a lateral resolution of $200 \mathrm{~nm}$ and are useful for generating secondary ions with a positive charge, so they are mostly used for the detection of metals. In contrast, $\mathrm{Cs}^{+}$currents achieve a much higher resolution - 50-100 $\mathrm{nm}$ - and are used for sputtering negatively charged ions, such as $\mathrm{C}^{-}, \mathrm{CN}^{-}, \mathrm{F}^{-}$, etc. In this study $\mathrm{I}$ employed $\mathrm{Cs}^{+}$primary ions to measure the isotopic 
composition of the samples. Nitrogen is detected in NanoSIMS as the molecular ion cyanide $\left(\mathrm{CN}^{-}\right)$because it is very difficult to ionize otherwise.

NanoSIMS requires placing the samples under ultrahigh vacuum $\left(10^{-8} \mathrm{~Pa}\right.$; Hoppe et al., 2013). This requirement of the NanoSIMS techniques precludes any possibility to perform live cell experiments. What is more, the samples have to be well fixed and embedded in nonvolatile materials. In NanoSIMS, the ion optical system that acts as a lens for collecting the sputtered secondary ions is very close to the sample surface (cca $400 \mu \mathrm{m}$ ). This reduces aberrations and improves the resolution of the technique, but, on the other hand, the proximity of the ion lens to the sample hinders the possibility of simultaneous imaging of the sample using optical microscopy (Hoppe et al., 2013).

In principle, due to the fact that NanoSIMS detects isotopes instead of fluorophores, the samples can be imaged in NanoSIMS in a label-free manner. This involves simply the detection of the isotopes and elements naturally present in the biological material. For instance, ${ }^{14} \mathrm{~N}$ is more abundant in proteins than in lipids. Therefore, if membranes are investigated using NanoSIMS the ${ }^{14} \mathrm{~N}$ signal should indicate where regions enriched in proteins are.

However, if metabolic features are of interest, stable isotopes that are present in low amounts in living material should be employed, such as ${ }^{13} \mathrm{C}(1.07 \%),{ }^{15} \mathrm{~N}(0.368 \%),{ }^{33} \mathrm{~S}(0.76 \%$; Rosman and Taylor, 1998). Such investigations are now of wide interest and power the field of NanoSIMS imaging (Lechene et al., 2006; Zhang et al., 2012; Frisz et al., 2013; Senyo et al., 2013; Steinhauser and Lechene, 2013). In addition, correlative NanoSIMS studies with other techniques such as super-resolution fluorescence microscopy (Saka et al., 2014b) and atomic force microscopy (Anderton et al., 2011) have been described. 


\subsection{Aims of the Project}

Both optical and isotopic super-resolution microscopy can reach a high resolution ( $30 \mathrm{~nm}$ in the lateral plane) and are now advancing towards visualizing molecular-sized complexes (Lechene et al., 2006; Toomre and Bewersdorf, 2010; Sengupta et al., 2012; Göttfert et al., 2013; Hoppe et al., 2013). In the case of fluorescence super-resolution microscopy, the labeling tools are lagging behind and the most widely used probes are still the ones developed for diffraction-limited microscopy. For NanoSIMS there are no isotopic probes for the specifically labeling one protein of interest in a manner analogous to GFP in fluorescence imaging. Hence it would be advantageous to identify and test smaller probes to accurately label proteins.

Non-canonical amino acids are increasingly being used as tags for labeling proteins in various applications ranging from FRET and crosslinking studies to super-resolution microscopy investigations (Davis and Chin, 2012; Neumann, 2012; Milles and Lemke, 2013). They can be coupled to virtually any probes and this makes them good markers for a wide variety of techniques.

The first goal of my project was to assess whether FP chimeras exhibit different nanostructural patterns compared with their non-tagged counterparts. FPs contributed significantly to our understanding of protein expression and localization (Chalfie et al., 1994) due to their ease of use, particularly for live cell applications (Chen et al., 2002; Frommer et al., 2009). FPs have not only revolutionized protein investigations in live cell microscopy, but have also found applications in pointillistic super-resolution microscopy settings (Annibale et al., 2011b; Sengupta et al., 2011). However, FP tagging may induce protein dimerization and oligomerization (Jain et al., 2001; Annibale et al., 2011a), protein mislocalization (Palmer and Freeman, 2004), and problems in protein-protein interaction (Hammond et al., 2010). The wide use of FPs as protein labels raises the point of the validation of these studies using smaller tags and super-resolution microscopy analysis. With this purpose in mind, I employed the incorporation of clickable ncAAs into specific proteins and visualized them in super-resolution microscopy.

The second goal of my project, involved designing a specific protein labeling paradigm for NanoSIMS. This technique is gaining momentum because it enables the visualization of up to seven isotopic labels in the sample with a resolution of $\sim 100 \mathrm{~nm}$. So far specific proteins were identified in NanoSIMS either through correlative fluorescence-NanoSIMS approaches 
(Saka et al., 2014b) or via antibodies directly labeled with metal ion probes (Angelo et al., 2014). However, these offer only a general labeling method and it is not possible to look at specific proteins or cell compartments without the aid of complementary methods, such as optical microscopy.

In this project, I aimed to gain insight into two basic questions: 1) does the presence on an FP tag induce alterations in the nanoscale organization protein assemblies? and 2) is there an equivalent of genetically encoded FPs for NanoSIMS measurements? 


\section{MATERIALS AND METHODS}

\subsection{Materials and Instruments}

\subsubsection{Manufacturers and Providers}

All the general compounds and specific reagents as well as the laboratory equipment used for this project were purchased from the companies or were provided by the institutions listed below (Table 2-1). In the rest of this work, whenever the companies or institutions will be mentioned, only the abbreviated name version will be used.

Table 2-1 Companies and institutions that have provided the materials and instruments

\begin{tabular}{|lll|}
\hline Company/Institution & City/State & Country \\
\hline Abberior GmbH & Göttingen & Germany \\
\hline Abcam & Cambridge & UK \\
\hline $\begin{array}{l}\text { ACD/Labs } \\
\text { Advanced Chemistry Development Inc.) }\end{array}$ & Toronto & Canada \\
\hline Addgene & Cambridge, MA & USA \\
\hline AHF & Tübingen & Germany \\
\hline Agilent Technologies & Santa Clara, CA & USA \\
\hline AppliChem GmbH & Darmstadt & Germany \\
\hline ATTO-TEC GmbH & Siegen & Germany \\
\hline $\begin{array}{l}\text { BD Biosciences } \\
\text { Becton Dickinson and Company) }\end{array}$ & Franklin Lakes, NJ & USA \\
\hline Beem Inc. & West Chester, PA & USA \\
\hline Branson Ultrasonics Corp. & Danbury, CT & USA \\
\hline Cameca & Genevilliers & France \\
\hline Carl Roth, GmbH + CoKG & Karlsruhe & Germany \\
\hline Cell Signaling & Beverly, MA & USA \\
\hline Chemdraw & Waltham, MA & USA \\
\hline Chroma Technology Corporation & Bellow Falls, VT & USA \\
\hline Clontech Laboratories Inc. & Saint-Germain-en-Laye & France \\
\hline Dianova & Hamburg & Germany \\
\hline
\end{tabular}


Company/Institution

City/State

European Molecular Biology Laboratory

(EMBL)

Eppendorf

Eurofins MWG Synthesis GmbH

Fujifilm

GE Healthcare

Gerhard Menzel GmbH

Gibco

Institute of Neuro- and Sensory Physiology

Institute for Organic and Biomolecular

Chemistry

Invitrogen

Jena Bioscience GmbH

Leica Microsystems GmbH

LI-COR Biosciences

Life Technologies

LIMES Institute

London Resin Company Ltd

Max Planck Institute for Biophysical

Chemistry (MPI-BPC)

Merck Millipore

Molecular Probes Inc.

Newport Spectra Physiscs GmbH

Novus Biologicals

Olympus Corporation

Qiagen

Santa Cruz Biotechnology

Scientific Volume Imaging

SiChem

(Sirius Fine Chemicals GmbH)

Sigma-Aldrich Chemie GmbH

Sigma-Aldrich Inc.

Struers

Synaptic Systems

(Synaptic Systems GmbH)

TCI Europe

Technical University Munich

The MathWorks Inc.
Heidelberg Germany

Hamburg Germany

Ebersberg Germany

Tokyo

Japan

UK

Little Chalfont UK

Braunschweig

Paisley

Germany

UK

Germany

Germany

USA

Germany

Germany

USA

USA

Germany

UK

Berkshire UK

Göttingen Germany

Darmstadt

Eugene, OR

Germany

USA

Germany

Darmstadt

UK

Japan

Netherlands

USA

Dallas, TX USA

Hilversum

Netherlands

Bremen

Germany

Schnelldorf

Germany

Saint Louis, MO

Germany

Ballerup

Denmark

Göttingen

Germany

Zwijndrecht

Belgium

Freising-Weihenstephan

Natick, MA

Germany

USA 


\begin{tabular}{|lll|}
\hline Company/Institution & City/State & Country \\
\hline Systat Software Inc. & San Jose, CA & USA \\
\hline Thermo Fischer Scientific Inc. & Waltham, MA & USA \\
\hline $\begin{array}{l}\text { University Medical Center Göttingen } \\
\text { (UMG) }\end{array}$ & Göttingen & Germany \\
\hline VWR & Hannover & Germany \\
\hline
\end{tabular}

\subsubsection{Reagents}

The chemical compounds and enzymes used throughout this project can be found in the below table (Table 2-2).

Table 2-2 List of chemicals

\begin{tabular}{|c|c|c|c|c|}
\hline \multirow{4}{*}{ 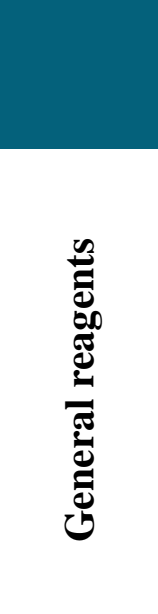 } & Product & Company/Institution & $\begin{array}{l}\text { Stock } \\
\text { (Solvent) }\end{array}$ & Final conc. \\
\hline & formic acid & Sigma-Aldrich & & \\
\hline & $\begin{array}{l}\text { 4',6'-diamino-2- } \\
\text { phenylindole·2HCl } \\
\text { (DAPI) }\end{array}$ & Molecular Probes & $\begin{array}{l}1 \mathrm{mg} / \mathrm{mL} \\
\left(\mathrm{H}_{2} \mathrm{O}\right)\end{array}$ & $1 \mu \mathrm{g} / \mathrm{mL}$ \\
\hline & $\begin{array}{l}\text { poly-L-lysine } \\
\text { hydrochloride (PLL) }\end{array}$ & $\begin{array}{l}\text { Sigma-Aldrich Chemie } \\
\text { GmbH }\end{array}$ & $\begin{array}{l}20 \mu \mathrm{g} / \mathrm{mL} \\
\left(\mathrm{H}_{2} \mathrm{O}\right)\end{array}$ & \\
\hline \multirow{2}{*}{ 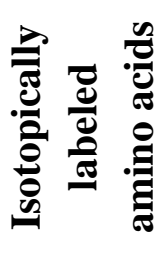 } & L-leucine-2- ${ }^{13} \mathrm{C}$ & Sigma-Aldrich & $\begin{array}{l}20 \mathrm{mg} / \mathrm{mL} \\
(\mathrm{PBS})\end{array}$ & $200 \mathrm{mg} / \mathrm{L}$ \\
\hline & L-leucine- $2-{ }^{15} \mathrm{~N}$ & Sigma-Aldrich & $\begin{array}{l}20 \mathrm{mg} / \mathrm{mL} \\
(\mathrm{PBS})\end{array}$ & $200 \mathrm{mg} / \mathrm{L}$ \\
\hline \multirow{3}{*}{ 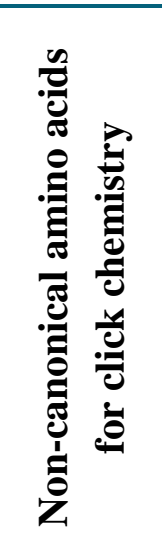 } & $\begin{array}{l}\text { 2-amino-6-(((2-azido- } \\
\text { ethoxy)- } \\
\text { carbonyl)amino)hexan } \\
\text { oic acid / azido-L- } \\
\text { lysine (AZK) }\end{array}$ & $\begin{array}{l}\text { Dr. Vladimir Belov } \\
\text { Mario Fischer } \\
\text { Max Planck Institute for } \\
\text { Biophysical Chemistry }\end{array}$ & & \\
\hline & $\begin{array}{l}\text { 4-azido-L- } \\
\text { phenylalanine } \\
\text { (pAzpa) }\end{array}$ & $\begin{array}{l}\text { Santa Cruz } \\
\text { Biotechnology }\end{array}$ & $\begin{array}{l}0.242 \mathrm{M} \\
(80 \% \mathrm{AcOH})\end{array}$ & $250 \mu \mathrm{M}$ \\
\hline & $\begin{array}{l}\text { bicyclo[6.1.0]nonyne- } \\
\text { L-lysine (BCNK) }\end{array}$ & $\begin{array}{l}\text { Dr. Edward Lemke, } \\
\text { EMBL }\end{array}$ & & $\begin{array}{l}250 \mu \mathrm{M} \text { or } \\
1 \mathrm{mM}\end{array}$ \\
\hline
\end{tabular}




\begin{tabular}{|c|c|c|c|c|}
\hline & Product & Company/Institution & $\begin{array}{l}\text { Stock } \\
\text { (Solvent) }\end{array}$ & Final conc. \\
\hline & $\begin{array}{l}\text { 2-amino-6-(((prop-2- } \\
\text { yn-1-yloxy)- } \\
\text { carbonyl)amino)hexan } \\
\text { oic acid / propargyl-L- } \\
\text { lysine (PRK) }\end{array}$ & $\begin{array}{l}\text { SiChem } \\
\text { Dr. Edward Lemke, } \\
\text { EMBL }\end{array}$ & $1 \mathrm{M}$ (DMSO) & $250 \mu \mathrm{M}$ \\
\hline & $\begin{array}{l}\text { N6-(((E)-cyclooct- } \\
\text { 2en-1- } \\
\text { yl)oxy)carbonyl)-L- } \\
\text { lysine / } \\
\text { strained cyclooctyne- } \\
\text { L-lysine (SCOK) }\end{array}$ & $\begin{array}{l}\text { Dr. Edward Lemke, } \\
\text { EMBL }\end{array}$ & & $\begin{array}{l}250 \mu \mathrm{M} \text { or } \\
1 \mathrm{mM}\end{array}$ \\
\hline & $\begin{array}{l}\text { N6-((((E)-cyclooct- } \\
\text { 2en-1- } \\
\text { yl)oxy)carbonyl)-L- } \\
\text { lysine / } \\
\text { trans-cyclooctene-L- } \\
\text { lysine (TCOK) }\end{array}$ & SiChem & & $1 \mathrm{mM}$ \\
\hline \multirow{7}{*}{ 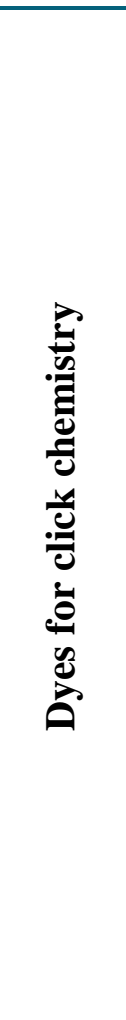 } & Atto647N-azide & ATTO-TEC GmbH & $\begin{array}{l}1 \mathrm{mM} \\
\text { (DMSO) }\end{array}$ & $2 \mu \mathrm{M}$ \\
\hline & $\begin{array}{l}\text { Alexa Fluor® 647- } \\
\text { azide, } \\
\text { triethylammonium } \\
\text { salt (Alexa647-azide) }\end{array}$ & Molecular Probes & $\begin{array}{l}5 \mathrm{mM} \\
(\mathrm{DMSO})\end{array}$ & $3 \mu \mathrm{M}$ \\
\hline & KK114-azide & \multirow{2}{*}{$\begin{array}{l}\text { Dr. Vladimir Belov } \\
\text { Dr. Kirill Kolmakov } \\
\text { Max Planck Institute for } \\
\text { Biophysical Chemistry }\end{array}$} & $\begin{array}{l}1 \mathrm{mM}(\mathrm{PBS} \\
\left.\text { or } \mathrm{H}_{2} \mathrm{O}\right)\end{array}$ & $\begin{array}{l}5 \mu \mathrm{M} \text { or } \\
50 \mu \mathrm{M}\end{array}$ \\
\hline & Star635P-azide & & $\begin{array}{l}1 \mathrm{mM}(\mathrm{PBS} \\
\left.\text { or } \mathrm{H}_{2} \mathrm{O}\right)\end{array}$ & $\begin{array}{l}25 \mu \mathrm{M} \text { or } \\
50 \mu \mathrm{M}\end{array}$ \\
\hline & $\begin{array}{l}\text { 3-(p-benzylamino)- } \\
\text { 1,2,4,5-tetrazine- } \\
\text { ATTO647N } \\
\text { (tetrazine-Atto647N) }\end{array}$ & Jena Bioscience & $\begin{array}{l}10 \mathrm{mM} \\
\text { (DMSO) }\end{array}$ & $\begin{array}{l}50 \mathrm{nM}, \\
100 \mathrm{nM} \text { or } \\
200 \mathrm{nM}\end{array}$ \\
\hline & tetrazine-KK114 & \multirow{2}{*}{$\begin{array}{l}\text { Dr. Vladimir Belov } \\
\text { Dr. Kirill Kolmakov } \\
\text { Max Planck Institute for } \\
\text { Biophysical Chemistry }\end{array}$} & & $\begin{array}{l}100 \mathrm{nM} \text { or } \\
200 \mathrm{nM}\end{array}$ \\
\hline & tetrazine-Star635 & & & $\begin{array}{l}100 \mathrm{nM} \text { or } \\
200 \mathrm{nM}\end{array}$ \\
\hline \multirow{4}{*}{ 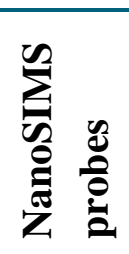 } & SK88-5/TriazNF2 & \multirow{4}{*}{$\begin{array}{l}\text { Selda Kabatas } \\
\text { Institute for Organic } \\
\text { and Biomolecular } \\
\text { Chemistry }\end{array}$} & \multirow{4}{*}{$\begin{array}{l}1 \mathrm{mM} \\
(\mathrm{DMSO})\end{array}$} & \multirow{4}{*}{$7 \mu \mathrm{M}$} \\
\hline & SK116/TriazNF3 & & & \\
\hline & SK134/TriazNF1 & & & \\
\hline & SK155 & & & \\
\hline
\end{tabular}




\begin{tabular}{|c|c|c|c|}
\hline & Product & Company/Institution & $\begin{array}{l}\text { Stock } \\
\text { (Solvent) }\end{array}$ \\
\hline \multirow{4}{*}{ 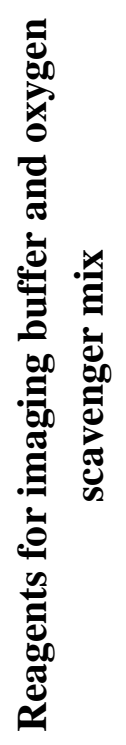 } & catalase (bovine liver) & \multirow{4}{*}{ Sigma-Aldrich Inc. } & \\
\hline & $\begin{array}{l}\text { glucose oxidase type } \\
\text { VII } \\
\text { (Aspergillus niger) }\end{array}$ & & \\
\hline & L-ascorbic acid & & \\
\hline & $\beta$-mercaptoethylamine & & \\
\hline \multirow{6}{*}{ 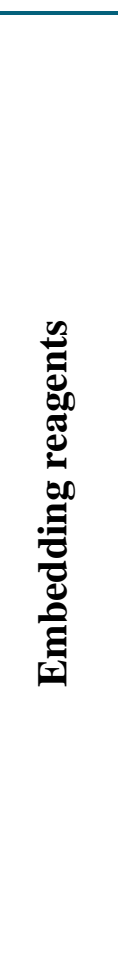 } & $\begin{array}{l}2,4,6- \\
\text { tris[bis(methoxymeth } \\
\text { yl)amino]-1,3,5- } \\
\text { triazine / melamine }\end{array}$ & TCI Europe & \multirow{2}{*}{$\begin{array}{l}1.344 \mathrm{~g} \text { melamine } \\
48 \mathrm{mg} \text { p-Toluenesulfonic } \\
\text { acid } \\
0.576 \mathrm{~mL} \text { distilled water }\end{array}$} \\
\hline & $\begin{array}{l}p \text {-toluenesulfonic acid } \\
\text { monohydrate }\end{array}$ & Sigma-Aldrich Inc. & \\
\hline & Epon resin & Struers & prepared from Eponfix kit \\
\hline & $\begin{array}{l}\text { LR White medium } \\
\text { grade resin }\end{array}$ & \multirow{2}{*}{$\begin{array}{l}\text { London Resin Company } \\
\text { Ltd. }\end{array}$} & \\
\hline & LR White accelerator & & \\
\hline & Mowiol 4-88 & $\begin{array}{l}\text { Merck Millipore, Merck } \\
\text { KGaA, Darmstadt, } \\
\text { Germany }\end{array}$ & $\begin{array}{l}24 \mathrm{~g} \text { glycerol } \\
9.6 \mathrm{~g} \text { Mowiol } 4-88 \\
62.4 \mathrm{~mL} \text { distilled } \\
\text { water } \\
9.6 \mathrm{~mL} 1 \mathrm{M} \text { Tris buffer }\end{array}$ \\
\hline
\end{tabular}




\subsubsection{Antibodies}

The antibodies employed in this project for Western blotting and immunostaining can be found in the Table 2-3 below.

Table 2-3 Primary and secondary antibodies used in blotting and cell stainings

\begin{tabular}{|llll|}
\hline Primary antibody & Company & Dilution & Application \\
\hline Rabbit anti-calnexin & Abcam & $1: 100$ & immunotaining \\
\hline Mouse anti-GM130 & BD Biosciences & $1: 100$ & immunotaining \\
\hline Mouse anti-TOMM20 & Sigma-Aldrich Inc. & $1: 100$ & immunotaining \\
\hline Mouse anti-ß-actin & Sigma-Aldrich Inc. & $1: 1000$ & immunoblot \\
\hline Mouse anti-GFP & Invitrogen & $1: 1000$ & immunoblot \\
\hline Mouse anti-VAMP2 & SySy & $1: 1000$ & immunoblot \\
\hline Secondary antibody & Company & Dilution & Application \\
\hline Goat anti-rabbit Cy2 & Dianova & $1: 100$ & immunotaining \\
conjugated & & $1: 100$ & immunotaining \\
\hline $\begin{array}{l}\text { Goat anti-mouse Cy3 } \\
\text { conjugated }\end{array}$ & Dianova & $1: 1000$ & immunoblot \\
\hline Goat anti-mouse & LI-COR Biosciences & & \\
\hline
\end{tabular}

\subsubsection{Buffers, Media and Solutions}

The buffers and solutions used in this project can be found in the Table 2-4 below.

Table 2-4 Buffers, media and solutions used in this project

\begin{tabular}{|ll|}
\hline Buffer/solution & Composition \\
\hline Anode buffer & $200 \mathrm{mM}$ Tris pH 8.9 \\
\hline Blocking buffer & PBS + 5\% milk powder (low fat) +0.1\% Tween-20 \\
\hline Cathode buffer & $100 \mathrm{mM}$ Tris, 100 mM Tricin, 1\% SDS \\
\hline DMEM (+) for BHK & $10 \%$ tryptose phosphate, 5\% fetal calf serum (FCS), 2 mM L- \\
\hline
\end{tabular}




\begin{tabular}{|c|c|}
\hline $\begin{array}{l}\text { Buffer/solution } \\
\text { cells }\end{array}$ & $\begin{array}{l}\text { Composition } \\
\text { glutamine, } 60 \mathrm{U} / \mathrm{mL} \text { penicillin and } 60 \mathrm{U} / \mathrm{mL} \text { streptomycin in } \\
\text { normal DMEM }\end{array}$ \\
\hline $\begin{array}{l}\text { DMEM (+) for COS- } \\
7 \text { cells }\end{array}$ & $\begin{array}{l}10 \% \text { FCS, } 4 \mathrm{mM} \mathrm{L} \text {-glutamine, } 60 \mathrm{U} / \mathrm{mL} \text { penicillin and } 60 \mathrm{U} / \mathrm{mL} \\
\text { streptomycin in normal DMEM }\end{array}$ \\
\hline Fixation reagent & $\begin{array}{l}0.2 \% \text { glutaraldehyde- } 4 \% \text { PFA in PBS or } \\
4 \% \text { PFA in PBS }\end{array}$ \\
\hline Gel buffer & $3 \mathrm{M}$ Tris, $0.3 \%$ SDS pH 8.45 \\
\hline Lysis buffer & 1\% SDS, 2 mM EDTA, 10 mM HEPES pH 7.4 \\
\hline $\begin{array}{l}\text { PBS } \\
\text { (phosphate-buffered } \\
\text { saline) }\end{array}$ & 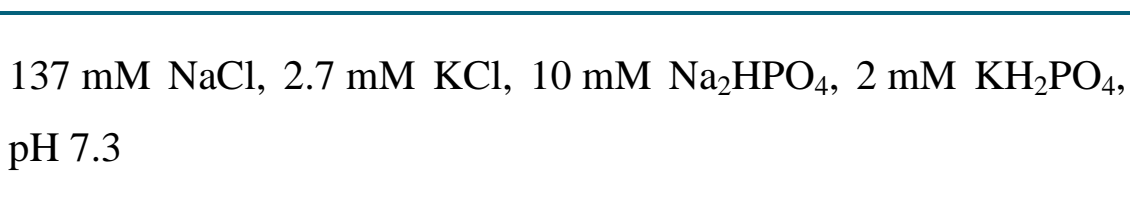 \\
\hline High-salt PBS & $500 \mathrm{mM} \mathrm{NaCl}, 20 \mathrm{mM} \mathrm{Na}_{2} \mathrm{HPO}_{4}, \mathrm{pH} 7.4$ \\
\hline $\begin{array}{l}\text { Permeabilization } \\
\text { solution }\end{array}$ & $0.1 \%$ Triton $X-100$ in PBS \\
\hline PLL & $20 \mu \mathrm{g} / \mathrm{mL}$ PLL ( producer) in water \\
\hline Quenching solution & $\begin{array}{l}100 \mathrm{mM} \mathrm{NH}_{4} \mathrm{Cl} \text { and } 100 \text { glycine in PBS or } \\
100 \mathrm{mM} \mathrm{NH}_{4} \mathrm{Cl}\end{array}$ \\
\hline Sample buffer & $\begin{array}{l}50 \mathrm{mM} \text { Tris, } 4 \% \text { SDS, } 0.01 \% \text { Serva Blue G, } 12 \% \text { glycerol, } 2 \% \beta- \\
\text { mercaptoethanol (pH 6.8) }\end{array}$ \\
\hline Sonication Buffer & $\begin{array}{l}120 \mathrm{mM} \text { monopotassium glutamate, } 20 \mathrm{mM} \text { potassium acetate, } \\
2 \mathrm{mM} \text { EGTA, } 20 \mathrm{mM} \text { HEPES-KOH, } \mathrm{pH} 7.2\end{array}$ \\
\hline Transfer buffer & $200 \mathrm{mM}$ glycin, $25 \mathrm{mM}$ Tris, $20 \%$ methanol, $0.04 \%$ SDS \\
\hline Wash buffer & PBS + 0.05\% Tween-20 \\
\hline
\end{tabular}




\subsubsection{Instruments and Equipment}

The instruments used in this study and the companies producing them are listed in Table 2-5.

Table 2-5 List of instruments and devices

\begin{tabular}{|c|c|c|}
\hline Instrument & Company & Applications \\
\hline Branson sonifier 450 & $\begin{array}{l}\text { Branson Ultrasonics } \\
\text { Corp. }\end{array}$ & $\begin{array}{l}\text { Generation of membrane sheets } \\
\text { by sonication }\end{array}$ \\
\hline $\begin{array}{l}\text { Leica pulsed STED } \\
\text { based on the inverted } \\
\text { confocal microscope TCS } \\
\text { SP5 }\end{array}$ & Microsystems & $\begin{array}{l}\text { Super-resolution imaging with a } \\
\text { resolution of up to } 50-60 \mathrm{~nm}\end{array}$ \\
\hline Leica SR GSD 3D & Microsystems & $\begin{array}{l}\text { Super-resolution imaging with a } \\
\text { resolution of up to } 20 \mathrm{~nm}\end{array}$ \\
\hline LI-COR infrared reader & LI-COR Biosciences & Imaging blotting membranes \\
\hline $\begin{array}{l}\text { Olympus IX71 } \\
\text { Microscope }\end{array}$ & Olympus & Epifluorescence imaging \\
\hline $\begin{array}{l}\text { Self-built STED setup by } \\
\text { Fabian Göttfert }\end{array}$ & --- & $\begin{array}{l}\text { Super-resolution imaging with a } \\
\text { resolution of up to } 30 \mathrm{~nm}\end{array}$ \\
\hline
\end{tabular}

\subsection{Gene Constructs}

The vector pCMV tRNA-PylRS WT was a kind gift from Dr. Edward Lemke (EMBL) and was employed in the herein experiments as previously described (Plass et al., 2012). The wild-type constructs presented in Table 2-6 were either purchased from Addgene or received from Prof. Dr. Reinhard Jahn and Dr. John Chua (Max Planck Institute for Biophysical Chemistry), Prof. Dr. Thorsten Lang (LIMES Institute), and Prof. Dr. Tiago Outeiro. The Amber stop codon mutants for 5-hydroxytryptamine receptor 1a (5HT1a) and insulin receptor were kindly provided by Dr. Marcus Niebert (Institute of Neuro- and Sensory Physiology, UMG) and Dr. Edward Lemke, respectively. 
Table 2-6 Original constructs, their reference sequence, organism of origin, and source

\begin{tabular}{|c|c|c|c|c|}
\hline Protein name & $\begin{array}{l}\text { Original } \\
\text { vector }\end{array}$ & Plasmid source & $\begin{array}{l}\text { Organism of } \\
\text { origin }\end{array}$ & RefSeq \\
\hline \multicolumn{5}{|c|}{ Wild-type constructs } \\
\hline \multirow{2}{*}{$\beta$-actin } & \multirow{2}{*}{$\begin{array}{l}\text { pEGFP } \\
\text { modified }\end{array}$} & \multirow{2}{*}{ Addgene 34839} & \multirow{2}{*}{ Homo sapiens } & NM_001101.3 \\
\hline & & & & NP_001092.1 \\
\hline \multirow{2}{*}{ amphiphysin } & \multirow{2}{*}{ pADTet } & \multirow{2}{*}{ Addgene 27692} & \multirow{2}{*}{ Mus musculus } & NM_175007.2 \\
\hline & & & & NP_778172.1 \\
\hline \multirow{2}{*}{$\mathbf{A P}-2 \mu$} & \multirow{2}{*}{ pcDNA3 } & \multirow{2}{*}{ Addgene 32752} & \multirow{2}{*}{$\begin{array}{l}\text { Rattus } \\
\text { norvegicus }\end{array}$} & NM_053837.1 \\
\hline & & & & NP_446289.1 \\
\hline \multirow{2}{*}{ complexin 1} & \multirow{2}{*}{ pET28a } & Prof. Dr. & Rattus & NM_022864.3 \\
\hline & & Reinhard Jahn & norvegicus & NP_074055.1 \\
\hline \multirow{2}{*}{ Doc2 $\alpha$} & \multirow{2}{*}{ pET-Doc $2 \alpha$} & Prof. Dr. & Rattus & NM_022937.2 \\
\hline & & Reinhard Jahn & norvegicus & NP_075226.1 \\
\hline \multirow{2}{*}{ Munc18-1 } & \multirow{2}{*}{ pcDNA3.1r } & \multirow{2}{*}{ Dr. John Chua } & \multirow{2}{*}{$\begin{array}{l}\text { Rattus } \\
\text { norvegicus }\end{array}$} & NM_013038.3 \\
\hline & & & & NP_037170.1 \\
\hline \multirow[t]{2}{*}{ PIPKI $\gamma$} & \multirow{2}{*}{ pEGFP-C2 } & \multirow{2}{*}{ Addgene 22299} & \multirow{2}{*}{ Homo sapiens } & NM_012398.2 \\
\hline & & & & NP_036530.1 \\
\hline \multirow{2}{*}{ Rab3a } & \multirow{2}{*}{ pET11d } & Prof. Dr. & Rattus & NM_013018.2 \\
\hline & & Reinhard Jahn & norvegicus & NP_037150.2 \\
\hline \multirow{2}{*}{ Rab5a } & \multirow{2}{*}{ pGEX-2T } & Prof. Dr. & Rattus & NM_022692.1 \\
\hline & & Reinhard Jahn & norvegicus & NP_073183.1 \\
\hline \multirow{2}{*}{ Rab7a } & \multirow{2}{*}{ pEGFP-C1 } & \multirow{2}{*}{ Addgene 12261} & \multirow{2}{*}{ Homo sapiens } & NM_004637.5 \\
\hline & & & & NP_004628.4 \\
\hline SNAP_23 & nGEX3 & Prof. Dr. & Rattus & NM_022689.2 \\
\hline & & Reinhard Jahn & norvegicus & NP_073180.1 \\
\hline SNAD 25 & nFYFP $\mathrm{Cl}$ & Prof. Dr. & Rattus & NM_030991.3 \\
\hline SNAT-ZS & PЕ ГГР-С & Thorsten Lang & norvegicus & NP_112253.1 \\
\hline SNAP_20 & nET28a & Prof. Dr. & Rattus & NM_053810.3 \\
\hline & & Reinhard Jahn & norvegicus & NP_446262.3 \\
\hline
\end{tabular}


Materials and Methods

\begin{tabular}{|c|c|c|c|c|}
\hline Protein name & $\begin{array}{l}\text { Original } \\
\text { vector }\end{array}$ & Plasmid source & $\begin{array}{l}\text { Organism of } \\
\text { origin }\end{array}$ & RefSeq \\
\hline synapsin Ia & pEGFP & $\begin{array}{l}\text { Ospedale San } \\
\text { Raffaele S.r.1 }\end{array}$ & $\begin{array}{l}\text { Rattus } \\
\text { norvegicus }\end{array}$ & $\begin{array}{l}\text { NM_019133.2 } \\
\text { NP_062006.1 }\end{array}$ \\
\hline synaptophysin & pGEX-KG & $\begin{array}{l}\text { Prof. Dr. } \\
\text { Reinhard Jahn }\end{array}$ & $\begin{array}{l}\text { Rattus } \\
\text { norvegicus }\end{array}$ & $\begin{array}{l}\text { NM_012664.2 } \\
\text { NP_036796.1 }\end{array}$ \\
\hline syntaptotagmin I & pCMV5 & $\begin{array}{l}\text { Prof. Dr. } \\
\text { Reinhard Jahn }\end{array}$ & $\begin{array}{l}\text { Rattus } \\
\text { norvegicus }\end{array}$ & $\begin{array}{l}\text { NM_001033680.2 } \\
\text { NP_001028852.2 }\end{array}$ \\
\hline syntaxin $1 a$ & pEYFP-N1 & $\begin{array}{l}\text { Prof. Dr. } \\
\text { Thorsten Lang }\end{array}$ & $\begin{array}{l}\text { Rattus } \\
\text { norvegicus }\end{array}$ & $\begin{array}{l}\text { NM_053788.2 } \\
\text { NP_446240.2 }\end{array}$ \\
\hline syntaxin 6 & $\begin{array}{l}\text { pQTEV- } \\
\text { STX6 }\end{array}$ & Addgene 31581 & Homo sapiens & $\begin{array}{l}\text { NM_005819.5 } \\
\text { NP_005810.1 }\end{array}$ \\
\hline syntaxin 7 & pcDNA3.1 & $\begin{array}{l}\text { Prof. Dr. } \\
\text { Reinhard Jahn }\end{array}$ & $\begin{array}{l}\text { Rattus } \\
\text { norvegicus }\end{array}$ & $\begin{array}{l}\text { NM_021869.2 } \\
\text { NP_068641.2 }\end{array}$ \\
\hline syntaxin 13 & pET28a & $\begin{array}{l}\text { Prof. Dr. } \\
\text { Reinhard Jahn }\end{array}$ & $\begin{array}{l}\text { Rattus } \\
\text { norvegicus }\end{array}$ & $\begin{array}{l}\text { NM_022939.2 } \\
\text { NP_075228.2 }\end{array}$ \\
\hline$\alpha$-synuclein & pEGFP-N3 & $\begin{array}{l}\text { Prof. Dr. Tiago } \\
\text { Outeiro }\end{array}$ & Homo sapiens & $\begin{array}{l}\text { NM_000345.3 } \\
\text { NP_000336.1 }\end{array}$ \\
\hline VAMP2 & pET-28a & $\begin{array}{l}\text { Prof. Dr. } \\
\text { Reinhard Jahn }\end{array}$ & $\begin{array}{l}\text { Rattus } \\
\text { norvegicus }\end{array}$ & $\begin{array}{l}\text { NM_012663.2 } \\
\text { NP_036795.1 }\end{array}$ \\
\hline VAMP4 & pEGFP-C3 & Addgene 42313 & Homo sapiens & $\begin{array}{l}\text { NP_003753.2 } \\
\text { NM_003762.4 }\end{array}$ \\
\hline Vtila- $\beta$ & pET28a & $\begin{array}{l}\text { Prof. Dr. } \\
\text { Reinhard Jahn }\end{array}$ & $\begin{array}{l}\text { Rattus } \\
\text { norvegicus }\end{array}$ & $\begin{array}{l}\text { NM_023101.1 } \\
\text { NP_075589.1 }\end{array}$ \\
\hline \multicolumn{5}{|c|}{ Amber mutants } \\
\hline 5HT1a & $\begin{array}{l}\text { pcDNA3.1 } \\
\text { HA-5HT1a } \\
\text { K101TAG } \\
\text { pcDNA3.1 } \\
\text { 5HT1a-GFP }\end{array}$ & $\begin{array}{l}\text { Dr. Marcus } \\
\text { Niebert }\end{array}$ & Mus musculus & $\begin{array}{l}\text { NM_008308.4 } \\
\text { NP_032334.2 }\end{array}$ \\
\hline
\end{tabular}




\begin{tabular}{|lllll|} 
Protein name & $\begin{array}{l}\text { Original } \\
\text { vector }\end{array}$ & Plasmid source & $\begin{array}{l}\text { Organism of } \\
\text { origin }\end{array}$ & RefSeq \\
\hline & K101TAG & & \\
insulin receptor & InER-GFP-N1 & & \\
(InsR) & K676TAG & Dr. Edward & Homo sapiens & NM_000208 \\
(Nikić et al., & LCI InsR & Lemke & NP_000199 \\
2014) & K676TAG & & \\
& IRES-CFP & & \\
\hline
\end{tabular}

\subsubsection{Constructs Obtained by Site-Directed Mutagenesis}

For the present experiments, SNAP-25 and VAMP2 coding sequences were first subcloned into pEGFP-N1 (Clontech) vector. The reason for this was to have a EGFP (from now on simply termed GFP) tag at the C-terminus of the protein of interest, which would act as a reporter for ncAA incorporation. This is particularly useful when testing the efficiency of ncAA incorporation for the different mutants obtained for one construct.

The coding sequences of SNAP-25 (in pEGFP-N1), syntaxin 1 (in pEYFP-N1), $\alpha$-synuclein (in pEGFP-N3), and VAMP2 (in pEGFP-N1) were subjected to site-directed mutagenesis to introduce Amber stop (TAG) codons using the QuikChange Site-Directed Mutagenesis Kit II (Agilent Technologies). The primers were designed based on the recommendations provided with this kit (refer to Table 2-7 for a list of primers used). To continue with, the mutagenesis protocols employed for the generation of Amber mutants respected the manufacturer's instructions.

For SNAP-25 a number of 9 mutants were designed and tested: Q66TAG, N68TAG, N77TAG, F84TAG, L203TAG, P207TAG, R212TAG, P215TAG, and M219TAG. Out of these, mutant F84TAG and M219TAG performed best in terms of incorporation efficiency and random GFP expression in cells that were transfected but no ncAA was added for incorporation. The latter relates to the random suppression of the Amber stop codon by other aminoacyl-tRNA pairs and to the reinitiation of translation from AUG start codons downstream of the Amber stop codon. 
Syntaxin 1 coding sequence was mutagenized to 10 different variants: L106TAG, F177TAG, I182TAG, V223TAG, V241TAG, V248TAG, V255TAG, Q258TAG, L289TAG, R294TAG. Out of all of these mutants, V255TAG incorporated ncAAs at the Amber stop codon with the best efficiency.

For $\alpha$-synuclein a number of 4 mutants were generated (G141TAG, T142TAG, S147TAG, and M151TAG) and G141TAG and T142TAG were selected as the best for ncAA incorporation experiments.

In the case of VAMP2, Amber stop codon was introduced at 4 different locations (R117TAG, I118TAG, S121TAG, R125TAG) the mutant R125TAG showed the highest incorporation yield and was therefore used in subsequent experiments. Consult Table 2-7 for details on the positions of the mutations and Figure 3-1 for the mutations selected for further experiments.

For the selected mutants (cloned in pEGFP-N1, pEGFP-N3 or pEYFP-N1), constructs lacking the C-terminal fluorescent protein (FP) region were generated and the resulting vectors were named $\mathrm{pN} 1$. The FP coding sequence was excised from the different constructs with the following restriction enzymes: AgeI and NotI for SNAP-25 and syntaxin 1, while SmaI and NotI were used for $\alpha$-synuclein and VAMP2. The linearized vectors without the FP gene insert were then extracted from agarose gel and extended to blunt ends with using the Klenow fragment polymerase. This was followed by religation using T4 ligase. Site-directed mutagenesis was employed to introduce of Ochre (TAA) stop codons after the protein coding sequence in the case of SNAP-25 and syntaxin 1 or immediately after the Amber stop codon for $\alpha$-synuclein and VAMP2.

Table 2-7 List of Amber mutants obtained through site-directed mutagenesis

\begin{tabular}{|lll} 
Protein & Mutation & Site-directed mutagenesis primers \\
& $\begin{array}{l}\text { Q66TAG } \\
\text { CAA } \rightarrow \text { TAG }\end{array}$ & CATTGAGGAAGGGATGGACTAGATCAATAAGGACATGAAAG \\
& CTTCATGTCCTTATTGATCTAGTCCATCCCTTCCTCAATG \\
\cline { 2 - 3 } & N68TAG & GGAAGGGATGGACCAAATCTAGAAGGACATGAAAGAAGCAG \\
\cline { 2 - 3 } & AAT $\rightarrow$ TAG & CTGCTTCTTTCATGTCCTTCTAGATTTGGTCCATCCCTTCC \\
\cline { 2 - 3 } & N77TAG & GACATGAAAGAAGCAGAAAAGTAGTTGACGGACCTAGGAAAATTC \\
& GAATTTCCTAGGTCCGTCAACTACTTTTCTGCTTCTTTCATGTC \\
\cline { 2 - 3 } & F84TAG & TGACGGACCTAGGAAAATAGTGCGGGCTTTGTGTGTG \\
& TTCTAG & CACACACAAAGCCCGCACTATTTTCCTAGGTCCGTCA \\
\hline
\end{tabular}




\begin{tabular}{|c|c|c|}
\hline Protein & Mutation & Site-directed mutagenesis primers \\
\hline & L203TAG & CAACGTGCAACAAAGATGTAGGGAAGTGGTCCGGTACCG \\
\hline & $\mathrm{CTG} \rightarrow \mathrm{TAG}$ & CGGTACCGGACCACTTCCCTACATCTTTGTTGCACGTTG \\
\hline & P207TAG & GCTGGGAAGTGGTTAGGTACCGCGGGCCC \\
\hline & $\mathrm{CCG} \rightarrow \mathrm{TAG}$ & GGGCCCGCGGTACCTAACCACTTCCCAGC \\
\hline & R212TAG & GTACCGCGGGCCTAGGATCCACCGGTC \\
\hline & $\mathrm{CGG} \rightarrow \mathrm{TAG}$ & GACCGGTGGATCCTAGGCCCGCGGTAC \\
\hline & P215TAG & GGCCCGGGATCCATAGGTCGCCACCATGG \\
\hline & $\mathrm{CCG} \rightarrow \mathrm{TAG}$ & CCATGGTGGCGACCTATGGATCCCGGGCC \\
\hline & M219TAG & CCGGTCGCCACCTAGGTGAGCAAGGGC \\
\hline & $\mathrm{ATG} \rightarrow \mathrm{TAG}$ & GCCCTTGCTCACCTAGGTGGCGACCGG \\
\hline \multirow{20}{*}{ 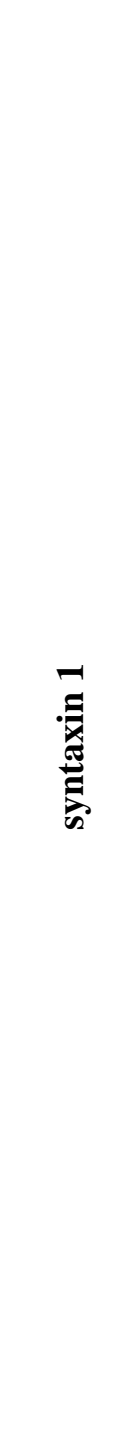 } & \multirow{2}{*}{$\begin{array}{l}\text { L106TAG } \\
\text { CTG } \rightarrow \text { TAG }\end{array}$} & GAGCAGGAGGAAGGTTAGAACCGCTCGTCGGCG \\
\hline & & CGCCGACGAGCGGTTCTAACCTTCCTCCTGCTC \\
\hline & \multirow{2}{*}{$\begin{array}{l}\text { F177TAG } \\
\text { TTT } \rightarrow \text { TAG }\end{array}$} & GGGAATCCCGCCATCTAGGCCTCTGGGATCATC \\
\hline & & GATGATCCCAGAGGCCTAGATGGCGGGATTCCC \\
\hline & \multirow{2}{*}{$\begin{array}{l}\text { I182TAG } \\
\text { ATC } \rightarrow \text { TAG }\end{array}$} & GCCATCTTTGCCTCTGGGATCTAGATGGACTCCAGCATCTCGAAG \\
\hline & & CTTCGAGATGCTGGAGTCCATCTAGATCCCAGAGGCAAAGATGGC \\
\hline & \multirow{2}{*}{$\begin{array}{l}\text { V223TAG } \\
\text { GTG } \rightarrow \text { TAG }\end{array}$} & GACATGGCCATGCTGTAGGAGAGCCAGGGGGAG \\
\hline & & CTCCCCCTGGCTCTCCTACAGCATGGCCATGTC \\
\hline & \multirow{2}{*}{$\begin{array}{l}\text { V241TAG } \\
\text { GTG } \rightarrow \text { TAG }\end{array}$} & CAATGTGGAACACGCTTAGGACTACGTGGAGAGGG \\
\hline & & СCCTCTCCACGTAGTCCTAAGCGTGTTCCACATTG \\
\hline & \multirow{2}{*}{$\begin{array}{l}\text { V248TAG } \\
\mathrm{GTG} \rightarrow \mathrm{TAG}\end{array}$} & CTACGTGGAGAGGGCCTAGTCTGACACCAAGAAGG \\
\hline & & CCTTCTTGGTGTCAGACTAGGCCCTCTCCACGTAG \\
\hline & \multirow{2}{*}{$\begin{array}{l}\text { V255TAG } \\
\text { GTC } \rightarrow \text { TAG }\end{array}$} & GTCTGACACCAAGAAGGCCTAGAAGTACCAGAGCAAGGCAC \\
\hline & & GTGCCTTGCTCTGGTACTTCTAGGCCTTCTTGGTGTCAGAC \\
\hline & \multirow{2}{*}{$\begin{array}{l}\text { Q258TAG } \\
\mathrm{CAG} \rightarrow \text { TAG }\end{array}$} & GCCGTCAAGTACTAGAGCAAGGCACGC \\
\hline & & GCGTGCCTTGCTCTAGTACTTGACGGC \\
\hline & \multirow{2}{*}{$\begin{array}{l}\text { L289TAG } \\
\text { TTG } \rightarrow \text { TAG }\end{array}$} & GGGCATCTTTGGATAGGTACCGCGGGCCC \\
\hline & & GGGCCCGCGGTACCTATCCAAAGATGCCC \\
\hline & \multirow{2}{*}{$\begin{array}{l}\text { R294TAG } \\
\mathrm{CGG} \rightarrow \mathrm{TAG}\end{array}$} & GGATTGGTACCGCGGGCCTAGGATCCACCGGTCGCCACC \\
\hline & & GGTGGCGACCGGTGGATCCTAGGCCCGCGGTACCAATCC \\
\hline
\end{tabular}




\begin{tabular}{|c|c|c|}
\hline Protein & Mutation & Site-directed mutagenesis primers \\
\hline \multirow{8}{*}{ 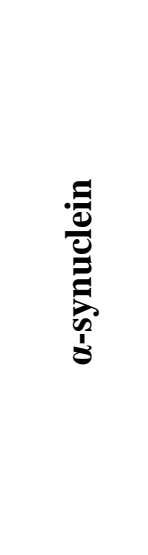 } & \multirow{2}{*}{$\begin{array}{l}\text { G141TAG } \\
\text { GGT } \rightarrow \text { TAG }\end{array}$} & GACTACGAACCTGAAGCCTAGACCGCGGGCCCGGGATCC \\
\hline & & GGATCCCGGGCCCGCGGTCTAGGCTTCAGGTTCGTAGTC \\
\hline & \multirow{2}{*}{$\begin{array}{l}\text { T142TAG } \\
\text { AAC } \rightarrow \text { TAG }\end{array}$} & GAACCTGAAGCCGGTTAGGCGGGCCCGGGATCC \\
\hline & & GGATCCCGGGCCCGCCTAACCGGCTTCAGGTTC \\
\hline & \multirow{2}{*}{$\begin{array}{l}\text { S147TAG } \\
\mathrm{TCC} \rightarrow \mathrm{TAG}\end{array}$} & GCGGGCCCGGGATAGATCGCCACCATG \\
\hline & & CATGGTGGCGATCTATCCCGGGCCCGC \\
\hline & \multirow{2}{*}{$\begin{array}{l}\text { M151TAG } \\
\text { ATG } \rightarrow \text { TAG }\end{array}$} & GGATCCATCGCCACCTAGGTGAGCAAGGGCGAG \\
\hline & & CTCGCCCTTGCTCACCTAGGTGGCGATGGATCC \\
\hline \multirow{8}{*}{$\sum_{i}^{N}$} & \multirow{2}{*}{$\begin{array}{l}\mathrm{R} 117 \mathrm{TAG} \\
\mathrm{CGA} \rightarrow \mathrm{TAG}\end{array}$} & TCATCGTTTACTTCAGCACTTAGATTCTGCAGTCGACGGTACC \\
\hline & & GGTACCGTCGACTGCAGAATCTAAGTGCTGAAGTAAACGATGA \\
\hline & \multirow{2}{*}{$\begin{array}{l}\mathrm{I} 118 \mathrm{TAG} \\
\mathrm{ATT} \rightarrow \mathrm{TAG}\end{array}$} & GTTTACTTCAGCACTCGATAGCTGCAGTCGACGGTACCG \\
\hline & & CGGTACCGTCGACTGCAGCTATCGAGTGCTGAAGTAAAC \\
\hline & \multirow{2}{*}{$\begin{array}{l}\mathrm{S} 121 \mathrm{TAG} \\
\mathrm{TCG} \rightarrow \mathrm{TAG}\end{array}$} & CTCGAATTCTGCAGTAGACGGTACCGCGGGC \\
\hline & & GCCCGCGGTACCGTCTACTGCAGAATTCGAG \\
\hline & \multirow{2}{*}{$\begin{array}{l}\text { R125TAG } \\
\mathrm{CGG} \rightarrow \text { TAG }\end{array}$} & GTCGACGGTACCGTAGGCCCGGGATCCAC \\
\hline & & GTGGATCCCGGGCCTACGGTACCGTCGAC \\
\hline
\end{tabular}

\subsubsection{Constructs Obtained by PCR Cloning}

For the rest of the proteins of interest, the coding sequences were inserted using PCR cloning into vector backbones with or without GFP (see Figure 3-4 for schematic depictions of the destination vectors). Refer to Table 2-8 for a comprehensive list of primers used. The destination vector backbones were obtained from pEGFP-N1 and pN1 VAMP2 R125TAG after the excision of the VAMP2 coding sequence. Both the vector backbones and the PCR products were cut with a suitable set restriction enzymes (see Table 2-8 for details on restriction enzyme combinations for each protein of interest). These enzymatic restrictions were performed in such a manner as to maintain the Amber mutation and GFP in the pEGFP-N1 vector, respectively the Amber and Ochre stop codons in pN1. The pairs of restricted vectors and PCR products were subjected to ligation using the T4 DNA ligase. 
Table 2-8 List of Amber mutants obtained through PCR cloning

\begin{tabular}{|c|c|c|c|}
\hline Protein name & $\begin{array}{l}\text { Amber } \\
\text { position }\end{array}$ & PCR cloning primers & $\begin{array}{l}\text { Restriction } \\
\text { sites }\end{array}$ \\
\hline$\beta$-actin & R457TAG & $\begin{array}{l}\text { attaGCTAGCATGGATGATGATATCGCC } \\
\text { attaGGTACCccGAAGCATTTGCG }\end{array}$ & $\begin{array}{l}\text { NheI } \\
\text { KpnI }\end{array}$ \\
\hline Amphiphysin & R692TAG & $\begin{array}{l}\text { attaGCTAGCATGGCCGACATCAAG } \\
\text { attaGTCGACcCCTCCAGGCG }\end{array}$ & $\begin{array}{l}\text { NheI } \\
\text { SalI }\end{array}$ \\
\hline $\mathbf{A P}-2 \mu$ & R441TAG & $\begin{array}{l}\text { attaGCTAGCATGATCGGAGGCTTATTC } \\
\text { attaGTCGACccGCAGCGG }\end{array}$ & $\begin{array}{l}\text { NheI } \\
\text { SalI }\end{array}$ \\
\hline Complexin 1 & R140TAG & $\begin{array}{l}\text { attaGCTAGCATGGAGTTCGTGATGAAAC } \\
\text { attaGTCGACccCTTCTTGAACATGTCC }\end{array}$ & $\begin{array}{l}\text { NheI } \\
\text { SalI }\end{array}$ \\
\hline $\operatorname{Doc} 2 \alpha$ & R409TAG & $\begin{array}{l}\text { attaGCTAGCATGAGGGGCCGC } \\
\text { attaGTCGACccGGCCAACGG }\end{array}$ & $\begin{array}{l}\text { NheI } \\
\text { SalI }\end{array}$ \\
\hline Munc18-1 & R600TAG & $\begin{array}{l}\text { attaGCTAGCATGGCCCCCATTGG } \\
\text { attaGTCGACccACTGCTTATTTCTTCGTC }\end{array}$ & $\begin{array}{l}\text { NheI } \\
\text { SalI }\end{array}$ \\
\hline PIPKI $\gamma$ & R674TAG & $\begin{array}{l}\text { attaAGATCTATGGAGCTGGAGGTACCG } \\
\text { attaGTCGACccTGTGTCGCTCTC }\end{array}$ & $\begin{array}{l}\text { BglII } \\
\text { SalI }\end{array}$ \\
\hline Rab3a & R226TAG & $\begin{array}{l}\text { attaGCTAGCATGGCCTCAGCCACAG } \\
\text { attaGTCGACccGCAGGCGCAATC }\end{array}$ & $\begin{array}{l}\text { NheI } \\
\text { SalI }\end{array}$ \\
\hline Rab5a & R134TAG & $\begin{array}{l}\text { attaGCTAGCATGTACTACCGAGGAGCAC } \\
\text { attaGTCGACccGTTACTACAACACTGGC }\end{array}$ & $\begin{array}{l}\text { NheI } \\
\text { SalI }\end{array}$ \\
\hline Rab7a & R125TAG & $\begin{array}{l}\text { attaGCTAGCATGACCTCTAGGAAGAAAGTG } \\
\text { attaGTCGACccGCAACTGCAG }\end{array}$ & $\begin{array}{l}\text { NheI } \\
\text { SalI }\end{array}$ \\
\hline
\end{tabular}


Materials and Methods

\begin{tabular}{|c|c|c|c|}
\hline Protein name & $\begin{array}{l}\text { Amber } \\
\text { position }\end{array}$ & PCR cloning primers & $\begin{array}{l}\text { Restriction } \\
\text { sites }\end{array}$ \\
\hline SNAP-23 & R216TAG & $\begin{array}{l}\text { attaGCTAGCATGGATGATCTATCACCAGAAG } \\
\text { attaGTCGACccGCTGTCAATGAGTTTC }\end{array}$ & $\begin{array}{l}\text { NheI } \\
\text { SalI }\end{array}$ \\
\hline SNAP-29 & R263TAG & $\begin{array}{l}\text { attaGCTAGCATGTCTGGCTATCCTAAAAGC } \\
\text { attaGTCGACccGAGTTGCCGC }\end{array}$ & $\begin{array}{l}\text { NheI } \\
\text { SalI }\end{array}$ \\
\hline synapsin Ia & R710TAG & $\begin{array}{l}\text { attaGCTAGCATGAACTACCTGCGG } \\
\text { attaGTCGACccGTCGGAGAAGAG }\end{array}$ & $\begin{array}{l}\text { NheI } \\
\text { SalI }\end{array}$ \\
\hline synaptophysin & R313TAG & $\begin{array}{l}\text { attaGCTAGCATGGACGTGGTGAATC } \\
\text { attaGTCGACccCATCTGATTGGAGAAG }\end{array}$ & $\begin{array}{l}\text { NheI } \\
\text { SalI }\end{array}$ \\
\hline $\begin{array}{l}\text { syntaptotagmin } \\
\text { I }\end{array}$ & R427TAG & $\begin{array}{l}\text { attaGCTAGCATGGTGAGTGCCAGTCATC } \\
\text { attaGTCGACccCTTCTTGACAGCCAG }\end{array}$ & $\begin{array}{l}\text { NheI } \\
\text { SalI }\end{array}$ \\
\hline syntaxin 6 & R261TAG & $\begin{array}{l}\text { attaGCTAGCATGTCCATGGAGGAC } \\
\text { attaGTCGACccCAGCACTAAGAAG }\end{array}$ & $\begin{array}{l}\text { NheI } \\
\text { SalI }\end{array}$ \\
\hline syntaxin 7 & R125TAG & $\begin{array}{l}\text { attaGCTAGCATGTCTTACACTCCGGG } \\
\text { attaGTCGACccGCCTTTCAGACC }\end{array}$ & $\begin{array}{l}\text { NheI } \\
\text { SalI }\end{array}$ \\
\hline syntaxin 13 & R280TAG & $\begin{array}{l}\text { attaGCTAGCATGTCCTACGGTCCC } \\
\text { attaGTCGACccCTTAGAAGCAACCC }\end{array}$ & $\begin{array}{l}\text { NheI } \\
\text { SalI }\end{array}$ \\
\hline VAMP4 & R147TAG & $\begin{array}{l}\text { attaGCTAGCATGCCTCCCAAGTTTAAG } \\
\text { attaGTCGACccAGTACGGTATTTCATG }\end{array}$ & $\begin{array}{l}\text { NheI } \\
\text { SalI }\end{array}$ \\
\hline Vti1a- $\beta$ & R230TAG & $\begin{array}{l}\text { attaGCTAGCATGTCAGCCGACTTCGAAG } \\
\text { attaGTCGACccGTGTCCTCTGACAAAAAAAG }\end{array}$ & $\begin{array}{l}\text { NheI } \\
\text { SalI }\end{array}$ \\
\hline
\end{tabular}




\subsection{Cell Culture}

\subsubsection{Cell Maintenance}

Baby hamster kidney-21 (here simply referred to as BHK) fibroblasts were used for the vast majority of the experiments included in this study, while COS-7 monkey fibroblasts were used for expressing syntaxin 1 for STED imaging. BHK cells were passaged every 1-4 days and propagated in DMEM (+) for BHK cells that contains Dulbecco's Modified Eagle Medium (DMEM; Life Technologies) supplemeted with 10\% tryptose phosphate, 5\% fetal calf serum (FCS), $2 \mathrm{mM} \mathrm{L-glutamine,} 60 \mathrm{U} / \mathrm{mL}$ penicillin and $60 \mathrm{U} / \mathrm{mL}$ streptomycin.

COS-7 cells were maintained in culture in DMEM (+) for COS-7 cells containing $10 \%$ FCS, $4 \mathrm{mM}$ L-glutamine, $60 \mathrm{U} / \mathrm{mL}$ penicillin and $60 \mathrm{U} / \mathrm{mL}$ streptomycin. This cell type was passaged every 2-5 days once they had reached $85-95 \%$ confluency.

\subsubsection{Plating Cells for Fluorescence Miscroscopy}

For fluorescence microscopy investigations, the cells were seeded on $12 \mathrm{~mm}$ coverslips coated with poly-L-lysine hydrochloride (PLL; Sigma-Aldrich Chemie GmbH) so that on the day of the transfection they would reach a confluency of $70-80 \%$.

\subsubsection{Plating Cells for Western Blotting}

For Western blotting experiments, BHK cells were seeded one day in advance on 6-well plates so that before transfection they would reach a confluency of 70-80\%.

\subsection{Treatments with Modified Amino Acids}

\subsubsection{Clickable Non-Canonical Amino Acids}

Approximately one hour before transfection, the medium of the cells was changed to medium without antibiotic (in controls) or to medium without antibiotic supplemented with the ncAA amino acid. Please refer to Table 2-2 for details on the ncAA stock solutions as well as their final concentrations used in the cell medium. 


\subsubsection{Isotopically Labeled Amino Acids}

Isotopically labeled analogues of leucine, L-leucine-2- ${ }^{15} \mathrm{~N}$ and L-leucine-2- ${ }^{13} \mathrm{C}$, were added to the cell medium for either one day or for three days, with medium exchanges every 24 hours. See Table 2-2 for details on the stock and final concentration in solution. For an experimental timeline of the SPILL experiments refer to Figure 2-1.

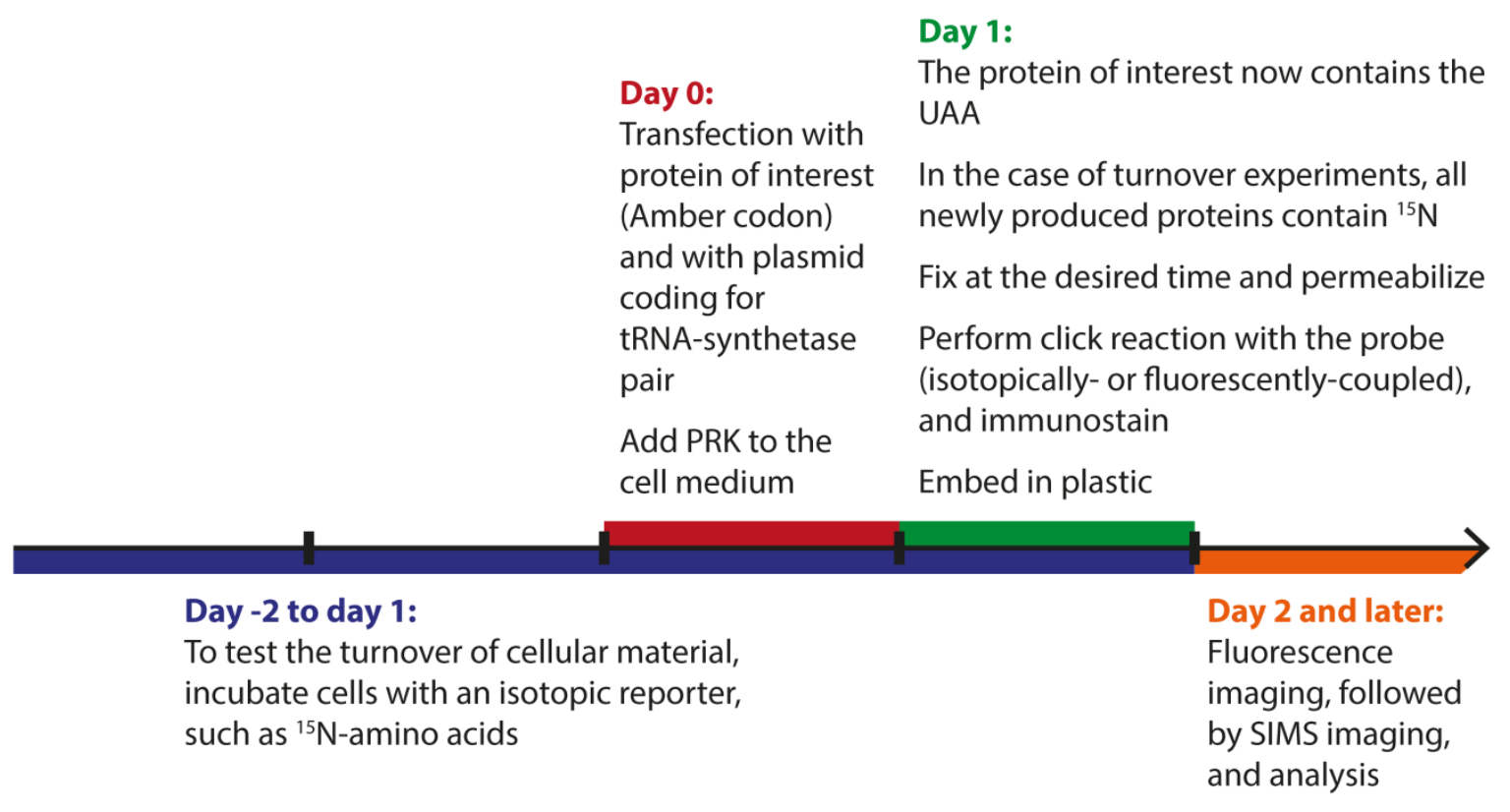

Figure 2-1 Experimental timeline for SPILL

\subsection{Transfection}

For ncAA incorporation, the cells were cotransfected with two plasmids: i) one encoding for the bioorthogonal aminoacyl-tRNA-synthetase (aaRS) and tRNA pair (RS/tRNA; refer to Table 2-9 for the specific pairs employed for a specific ncAA) and ii) a plasmid containing the gene of interest mutated to have an Amber stop codon (refer to Section 2.2 for details on how these plasmids were generated). These plasmids were mixed in a 1:1 ratio with a total amount of DNA of $2 \mu \mathrm{g}$ per well of a 12-well plate (for cells plated on $12 \mathrm{~mm}$ coverslips for fluorescence microscopy studies) or $5 \mu \mathrm{g}$ per well of a 6 -well plate (in Western blotting experiments). The transfection reagent Lipofectamine 2000 (Life Technologies) was mixed with the DNA according to the instructions of the manufacturer: $3 \mu \mathrm{L}$ (for a 12-well plate), respectively $5 \mu \mathrm{L}$ (for a 6-well plate) of Lipofectamine were allowed to equilibrate in $50 \mu \mathrm{L}$, 
or $100 \mu \mathrm{L}$ Opti-MEM (Gibco), respectively. The DNA was added in corresponding volumes of Opti-MEM. The DNA and Lipofectamine were mixed and incubated for 20 minutes at room temperature, before they were added to the cells. The expression of the proteins of interest and incorporation of the ncAAs were allowed to proceed for approximately 18 hours at $37^{\circ} \mathrm{C}$. The next day, the cells were briefly rinsed with PBS and incubated for about 2 hours in medium without antibiotic to ensure that the ncAA excess is washed off. The cells were again briefly washed in PBS before proceeding to fixation or sonication.

Table 2-9 Plasmid systems with the RS/tRNA pair for ncAA incorporation

\begin{tabular}{|lll|} 
ncAA & Plasmid for incorporation & Reference \\
\hline azido-L-lysine (AZK) & pCMV PylRS/tRNA WT & Plass et al., 2012 \\
\hline $\boldsymbol{p}$-azido-L-lysine (pAzpa) & pcpAzpaRS variant 2 & Hino et al., 2012 \\
\hline propargyl-L-lysine (PRK) & pCMV PylRS/tRNA WT & Plass et al., 2012 \\
\hline bicyclo [6.1.0] nonyne-L-lysine (BCNK) & pCMV PylRS/tRNA AF & Plass et al., 2012 \\
\hline strained cyclooctyne-L-lysine (SCOK) & pCMV PylRS/tRNA AF & Plass et al., 2012 \\
\hline trans-cyclooctene-L-lysine (TCOK) & pCMV PylRS/tRNA AF & Plass et al., 2012 \\
\hline
\end{tabular}

For Western blotting, the transfection was performed with $5 \mu \mathrm{g}$ DNA $(2.5 \mu \mathrm{g}$ pCMV PylRS/tRNA WT and $2.5 \mu \mathrm{g}$ pEGFP-N1 VAMP2-GFP Amber mutants) using $5 \mu \mathrm{L}$ Lipofectamine. The cells were allowed to incorporate either PRK or AZK (both at $250 \mu \mathrm{M}$ final concentration) for 18 hours. Controls where no ncAA was added were also performed. All cells were washed briefly in PBS and then incubated in normal DMEM before proceeding to lysis and preparation for SDS-PAGE and Western blotting.

\subsection{Membrane Sheet Generation}

BHK or COS-7 cells were grown on PLL-coated coverslips for 2-3 days up to a $80-95 \%$ confluence. Approximately 20 hours before sonication, the cells had been transfected as described in Section 2.5. Shortly before sonication, the cells were rinsed in PBS and then placed in a dish filled with ice-cold KGlu buffer. Refer to Figure 2-2 for an image of the sonication setup. 


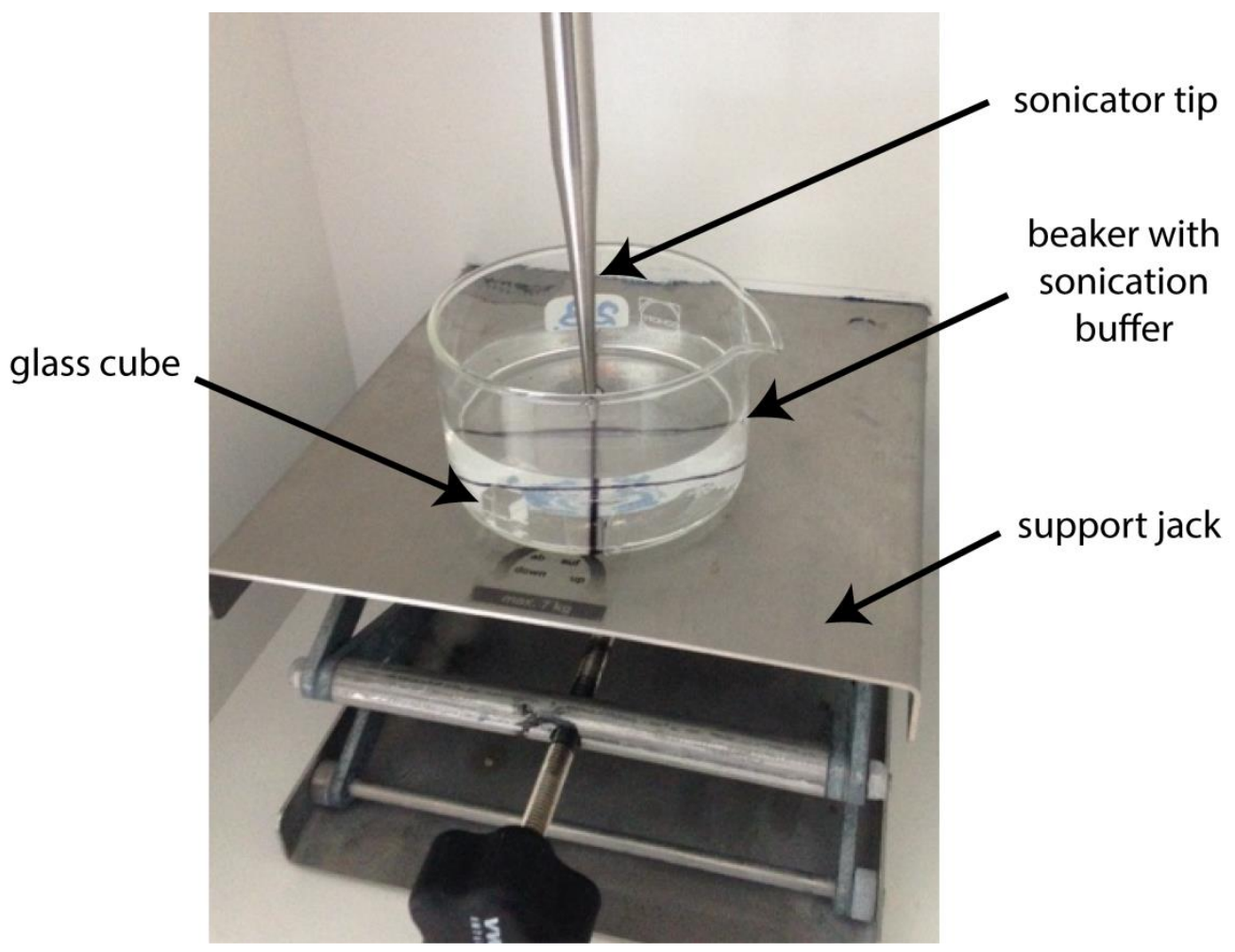

\section{Figure 2-2 The sonication setup}

The coverslip with adhered cells on the upper side was positioned in the center of the beaker containing ice-cold buffer with cells. The beaker is then placed on the support jack and the distance between the sonication tip and the coverslip is adjusted using the 1-cm glass cube as a reference. The latter is also used to stabilize the coverslip into place. For BHK and COS-7 cells, a 100-millisecond (1/10 of a second) sonication pulse was applied at 55-65\% power.

\subsection{Cell Fixation and Permeabilization}

\section{For Click Reactions}

Samples were fixed for 30 minutes with $0.2 \%$ glutaraldehyde and 4\% PFA in PBS or with 4\% PFA in PBS alone, then quenching was performed for 20 minutes in $100 \mathrm{mM} \mathrm{NH}_{4} \mathrm{Cl}$ and $100 \mathrm{mM}$ glycine in PBS or in $100 \mathrm{mM} \mathrm{NH}_{4} \mathrm{Cl}$, respectively. After a brief wash in PBS, the cells were permeabilized $0.1 \%$ Triton X-100 (in PBS), and then blocked for 15 minutes with $5 \%$ BSA and $5 \%$ peptone (in $0.1 \%$ Triton-X 100-PBS). The samples were incubated with $3 \%$ BSA in PBS briefly before the click reaction.

\section{For Immunostainings}

The cells were fixed with $0.2 \%$ glutaraldehyde and 4\% PFA in PBS for 30 minutes and quenched in $100 \mathrm{mM} \mathrm{NH}_{4} \mathrm{Cl}$ for 20 minutes. The samples were briefly washed in PBS and 
permeabilized with $1.5 \%$ BSA in $0.1 \%$ Triton X-100-PBS for 15 minutes (3 solution exhanges). The samples were incubated with 3\% BSA in PBS briefly before the click reaction.

\subsection{Labeling Protocols}

\subsubsection{Click Reactions}

\section{Copper-Catalyzed Click Reaction}

The click incubation mix was freshly prepared according to the manufacturer's protocol (Click-iT® Cell Reaction Buffer Kit, Life Technologies, cat. no. C10269). It contained 1× cell reaction buffer (component A), $2 \mathrm{mM} \mathrm{CuSO}_{4}$ (component B), a 10x dilution of Click-iT reaction buffer additive (component $\mathrm{C}$ ), and variable concentrations of azide-derivatized dyes or isotopic probes. The cells were incubated with the reaction mix for 30 minutes at $\mathrm{RT}$, in a dark humidified chamber. Each figure legend contains information about the final concentration of dye used.

\section{Copper-Free Click Reaction}

Click reactions in the absence of metal catalyzers were performed by diluting azide or tetrazine derivatives of fluorescent dyes into 1-1.5\% FCS in PBS as a blocking agent. The samples were fixed and permeabilized as described in Section 2.7. The exact concentrations for the dyes used are mention in the figure legends of each figure.

\subsubsection{Immunostainings}

Primary and secondary antibodies used in immunostaining and the final concentration used for protein labeling are shown in Table 2-3. All antibodies were incubated for 1 hour at room temperature with the samples. Between primary and secondary antibody application a 15minute washing step (3 buffer exchanges) with $0.1 \%$ Triton X-100 in PBS containing $1.5 \%$ BSA was performed. After the secondary antibody incubation, the cells were subjected to two PBS washes, one high-salt PBS wash, followed by another PBS wash (5 minutes each). 


\subsubsection{Staining of Nuclei with DAPI}

For epifluorescence microscopy, the nuclei were revealed with the DAPI reagent by incubating samples for 5 minutes in a DAPI solution (see Table 2-1) after fixation and click reaction. The unbound DAPI was then removed with three PBS washes and afterwards the samples were embedded in Mowiol.

\subsection{Plastic Embedding}

\subsubsection{Embedding in Melamine}

For STED and GSDIM applications, the cells were embedded in melamine $(2,4,6-$ Tris[bis(methoxymethyl)amino]-1,3,5-triazine, TCI Europe), as described previously by (Punge et al., 2008). The samples were incubated initially for 24 hours at room temperature (on silica gel for dehydration to occur) with melamine, to allow melamine penetration into the cells. Afterwards, the samples were moved to $40^{\circ} \mathrm{C}$ for 24 hours, followed by a further 48 hours at $60^{\circ} \mathrm{C}$. The melamine-embedded samples where cut using an ultramicrotome into 70-100 nm thin sections EM UC6 ultramicrotome (Leica Microsystems). The sections were either placed on microscope coverslips for later GSDIM imaging or further embedded in Mowiol.

\subsubsection{Embedding in LR White}

In the case of NanoSIMS measurements, the samples were embedded in LRWhite. Prior to this, they were fixed again with $0.2 \%$ glutaraldehyde-4\% PFA in PBS for 30 minutes and quenched with $100 \mathrm{mM} \mathrm{NH}_{4} \mathrm{Cl}$ and $100 \mathrm{mM}$ glycine in PBS for 20 minutes. After 15-minute washing step with PBS ( $2 \times$ buffer exchange), the samples were first dehydrated in ethanol solutions of increasing concentration and finally embedded in LRWhite, following the protocol described by (Saka et al., 2014b) . The resulting samples were cut into $200 \mathrm{~nm}$ thick sections samples (using an EM UC6 ultramicrotome) and placed on a silicone wafer. 


\subsection{Fluorescence Imaging}

\subsubsection{Epifluorescence Microscopy}

For epifluorescent imaging, an inverted fluorescence microscope (Olympus IX 71, see Table 2-5) was used with a $20 \times$ objective (Olympus, 0.50 NA). Sample illumination was provided by a $100 \mathrm{~W}$ mercury lamp (Olympus) and the fluorescence was recorded by a chargecoupled device (CCD) camera (FView II, Olympus). The images were taken using the CellF software (Olympus). Consult Table 2-10 for a list of the filters used during sample imaging. All images within figures were scaled identically, unless otherwise stated in the figure legend.

Table 2-10 Filter sets used for the Olympus epifluorescence microscope

\begin{tabular}{|lllll|}
\hline Filter & Excitation filter & Emission filter & Beamsplitter & Manufacturer \\
\hline DAPI & 350/50 D & $460 / 50 \mathrm{D}$ & $400 \mathrm{DCLP}$ & Olympus \\
\hline FITC & $480 / 40 \mathrm{HQ}$ & $527 / 30 \mathrm{HQ}$ & $505 \mathrm{LP} Q$ & Chroma \\
\hline Cy5 & $620 / 60 \mathrm{HQ}$ & $700 / 75 \mathrm{HQ}$ & $660 \mathrm{LP} Q$ & Chroma \\
\hline
\end{tabular}

\subsubsection{Confocal Microscopy}

Confocal images were acquired using a Leica TCS SP5 microscope (Leica Microsystems) equipped with a 100x oil objective (1.4 NA HCX PL APO CS; Leica). The KK114 and Star635P fluorophores in the samples were excited with a helium-neon laser at $633 \mathrm{~nm}$, while a $488 \mathrm{~nm}$ argon laser was employed for Cy2 excitation. An acousto-optic tunable filter (AOTF; Leica) enabled the selection of the appropriate emission intervals and photomultiplier tubes detected the signal. Images were acquired at a zoom of $7.5 \times$ using a $1,000 \mathrm{~Hz}$ line scan of a $20.68 \times 20.68 \mu \mathrm{m}$ area. This rendered an image of $1024 \times 1024$ pixels with a pixel size of $20.21 \times 20.21 \mathrm{~nm}$. 


\subsubsection{STED Microscopy}

For images taken at the commercial Leica STED setup (see Figure 3-10 and Figure 3-17), the same parameters as described above (Section 2.10.2) were employed, with the exception of the excitation and depletion lasers which consisted of a 635 diode laser for excitation of red-shifted fluorophores and the $750 \mathrm{~nm}$ MaiTai multiphoton laser (Newport SpectraPhysics).

The images taken at the setup built by Fabian Göttfert respected a protocol previously described (Göttfert et al., 2013). The microscope used a pulsed diode laser (at $640 \mathrm{~nm}$ wavelength) emitting $70 \mathrm{~ns}$ pulses to excite the fluorophores. A STED beam $(775 \mathrm{~nm}$ wavelength) passes a vortex plate to be circularly polarized to resemble a "doughnut" having a central zero. The STED beam allows fluorescence emission to occur only in a subdiffraction area around the zero intensity point. The pulse energies were of $7.5 \mathrm{~nJ}$ for STED and $0.4 \mathrm{pJ}$ for excitation and resulted in an effective PSF of approximately $30 \mathrm{~nm}$ (in diameter). This also corresponds to the attained resolution. For the the excitation laser, the pulse repetition rate was set to $1.1 \mathrm{MHz}$ to reduce photobleaching by relaxation of the triplet state (Donnert et al., 2007). The STED pulses that did not come in a short time (1-10 ns) after excitation pulses were blocked by an electrooptic modulator. This is also known as time gating and considerably reduces the dark count noise. For all the images, the pixel size and dwell time were set to $12 \mathrm{~nm}$ and $3 \mathrm{~ms}$, respectively.

\subsubsection{GSDIM Microscopy}

The GSDIM images were acquired at a Leica GSDIM microscope. For the images see Figure 3-20 and for the GSDIM setup refer to Table 2-5. The microscope was equipped with a 160x oil objective (Leica GSDIM) directly coupled to the sample stage and optimized for single fluorophore detection. GSDIM imaging was accomplished according to the manufacturer's instructions by exciting AlexaFluor647 with a $642 \mathrm{~nm}$ laser sent onto the sample at an angle between an epi and a TIRF illumination mode. For some of the measurements, a secondary $405 \mathrm{~nm}$ laser light was used to increase the switching activation rate. Fluorescence emission was triggered with an oblique illumination of the sample (i.e. it reduced the background) and the detection was performed using an sCMOS camera (PCO Edge monochrome). The exposure time for the camera was $10 \mathrm{~ms}$. 


\subsection{SDS-PAGE and Western Blotting}

For SDS-PAGE, the protocol established by Schägger and von Jagow, 1987 was employed. The cells were briefly rinsed with PBS and then $200 \mu \mathrm{L}$ pre-heated lysis buffer (see Table 2-4) were added in each well. The lysis buffer was freshly supplemeted with protease inhibitors (1:1000 aprotinin, 1:1000 leupeptin, 1:1000 pepstatin and 1:100 PMSF). The cells were scraped, mixed with an equal volume of $2 \times$ sample buffer (Table 2-4) and boiled for 510 minutes at $95^{\circ} \mathrm{C}$. After this step, the samples were either directly run on a gel or stored at $-20^{\circ} \mathrm{C}$. The proteins in the samples were separated on a $10 \%$ Tris/Tricine polyacrylamide gel. The proteins were afterwards transferred on a nitrocellulose membrane for 2 hours at $0.5 \mathrm{~A}$ in a wet blotting tank (Bio-Rad). When the transfer was over, the membranes were incubated for 30 minutes in blocking buffer and then for a further 1 hour with the primary antibodies. Following a 15-minute washing step ( $2 \times$ buffer exchanges), the membranes were incubated with the secondary antibodies (in blocking buffer) either for one more hour at room temperature or overnight at $4^{\circ} \mathrm{C}$. Details about the primary and the secondary antibodies used can be found in Table 2-3. Before imaging at the LI-COR instrument, the membranes were washed for 45 minutes ( $2 \times$ buffer exchanges) in wash buffer.

\subsection{Sample Preparation for SPILL Measurements}

\subsection{NanoSIMS Measurements}

Both the TriazNF1 and the SK155 samples were analyzed with a NanoSIMS 50L instrument (Cameca, Gennevilliers, France): The sputtering of ions in the samples was achieved with a $\mathrm{Cs}^{+}$primary ion beam at $16 \mathrm{keV}$ energy and a primary ion current in the range of $\sim 1-2 \mathrm{pA}$. Before each measurement $\mathrm{Cs}^{+}$ions were implantated into the sample to promote ionization efficiency.The generated secondary ion beam was mass-separated with a reliable resolution for potential isobars and detected using electron multipliers. Areas of $10 \times 10 \mu \mathrm{m}$ were scanned and rendered in a $512 \times 512$ pixel format. The dwell-time per pixel was $10 \mathrm{~ms}$.

For TriazNF1, The following ions of interest were detected: ${ }^{19} \mathrm{~F}^{-},{ }^{12} \mathrm{C}^{14} \mathrm{~N}^{-},{ }^{32} \mathrm{~S}^{-}$, mentioned throughout this dissertation as ${ }^{19} \mathrm{~F},{ }^{14} \mathrm{~N},{ }^{15} \mathrm{~N}$, and ${ }^{32} \mathrm{~S}$, respectively. 
For SK155, multiple plane measurements at different depths were performed and final summed images were obtained using a Matlab routine. The following ions were detected: ${ }^{19} \mathrm{~F}^{-},{ }^{12} \mathrm{C}^{14} \mathrm{~N}^{-},{ }^{12} \mathrm{C}^{15} \mathrm{~N}^{-}$. The images were binned using a $2 \times 2$ binning procedure for presentation purposes.

\subsection{Image Analysis and Processing}

\subsubsection{Data Analysis for Epifluorescence Images}

In Figure 3-13 and Figure 3-14, the fluorescence intensities in different channels were obtained using an automated Matlab routine written by Prof. Dr. Silvio O. Rizzoli. The regions of interest were selected automatically by using an empirically-derived threshold for the DAPI images as a reference. In each of these regions, the fluorescence of the green and red channels was measured and corrected for background. The latter was determined as the average intensity of the extracellular regions.

\subsubsection{Data Analysis for STED and GSDIM Images}

All data analysis for cluster sizes of proteins with or without FP tags was performed starting from images that were analyzed using Matlab (The Mathworks Inc.) routines written by Prof. Dr. Silvio O. Rizzoli. In each image, a region of interest (i.e. labeled cell or membrane sheet) was manually selected and using the Matlab software three parameters that relate to the size of individual protein assemblies were assessed. This involved spot size investigations in which the full width at half-maximum (FWHM) was measured. Peak intensity and total intensity for each spot were employed as parameters for estimating the brightness of each spot. Refer to Figure 2-3 for an exemplification of how these parameters were derived.

In Figure 3-18 (STED) and Figure 3-19 (GSDIM), the detected spots were subjected to a standardized analysis using a Matlab routine that normalizes the properties of the detected dots to the no ncAA controls and eliminates the values that are lower than 1SD (1 standard deviation) above the mean value of the few dots detected in the control. The latter consists of cells that were transfected with the same constructs but for which the ncAA was not provided in the medium. These control samples were subjected to the same click reaction 


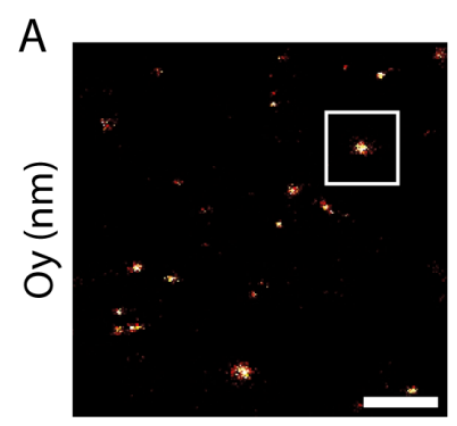

$\mathrm{Ox}(\mathrm{nm})$

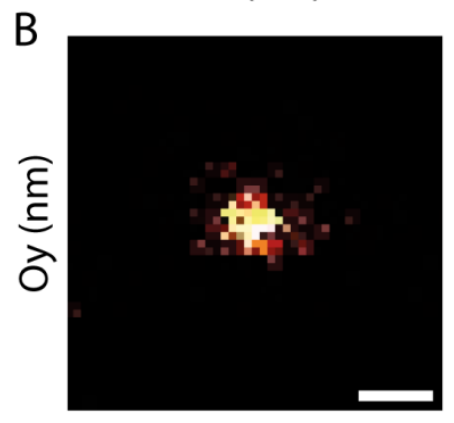

$\mathrm{Ox}(\mathrm{nm})$

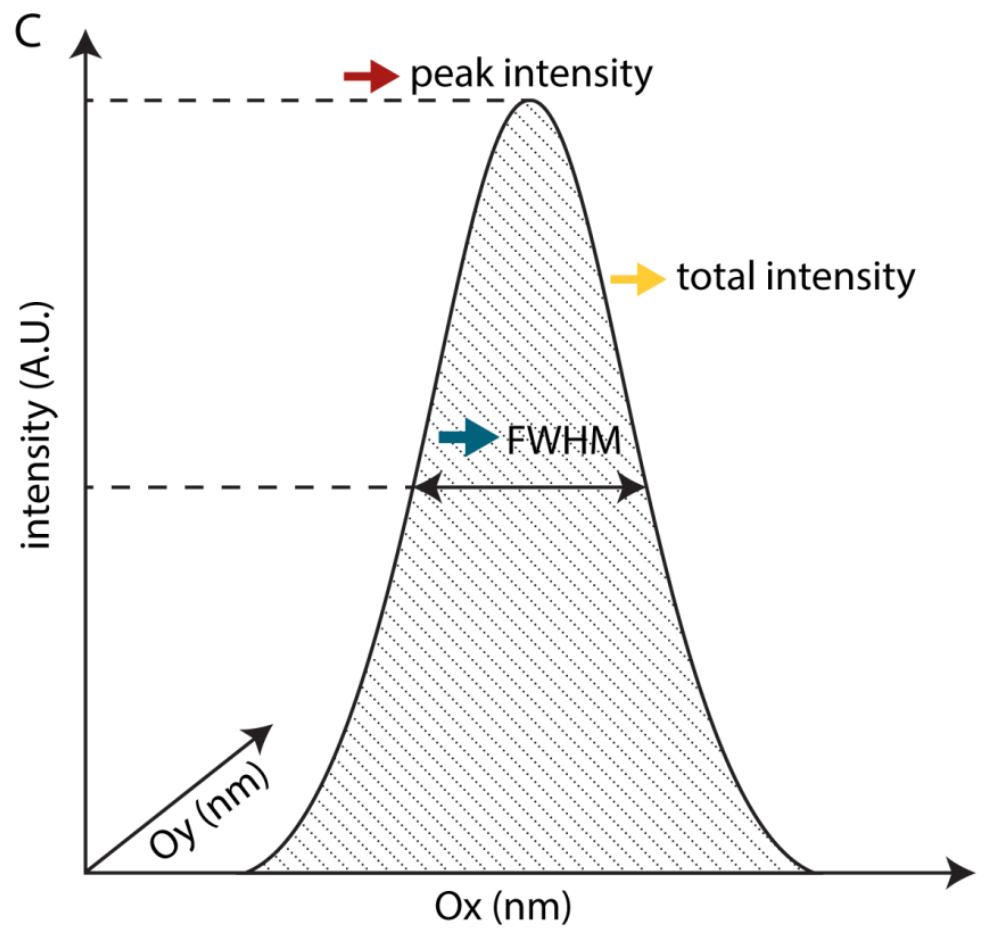

Figure 2-3 Parameters considered in data analysis from STED and GSDIM images.

In STED and GSDIM imaging the assemblies formed by the proteins of interest were detected as spots. A-B. Example image of data acquired using a GSDIM nanoscope (A) and an inset of a protein assembly (or cluster, B). C. During image analysis, the regions of interest were manually selected and the spots within these regions were automatically analyzed using Matlab routines for the following parameters: the full width at half maximum (FWHM) for each spot, which represents an estimate of the spot size, as well as the peak intensity and summed intensity for each spot, which represent two different ways to measure spot brightness. Scale bars for $\mathbf{A}$ and $\mathbf{B}, 250 \mathrm{~nm}$ and $50 \mathrm{~nm}$, respectively.

mix as the samples containing the PRK labeled proteins of interest. Therefore, the obtained spots (above 1SD) should represent true protein assemblies. These linescans of these spots were fitted by Gaussian curves and these fits were used to derive the spot size, peak intensity and the total intensity (Figure 2-3; see discussion above).

\subsubsection{Data Analysis for NanoSIMS Measurements}

Confocal and NanoSIMS images were overlaid and corrected for drifts and rotational misalignments before proceeding to image analysis.

In Figure 3-29 and Figure 3-30, manual selection 9 pixels circular region (in diameter) in the images of each sample was carried out. The average fluorescence intensity, or the average isotopic counts, were determined, and plotted. 
In Figure 3-30 C, the ratio between ${ }^{19} \mathrm{~F}$ and ${ }^{14} \mathrm{~N}$ was used and not the raw levels of ${ }^{19} \mathrm{~F}$ because the former is less prone to experimental variations in NanoSIMS measurements.

\subsection{Calculating TriazNF1 Copy Numbers}

For cells that expressed the proteins of interest and were labeled with TriazNF1, the isotopic counts for ${ }^{14} \mathrm{~N}$ and ${ }^{15} \mathrm{~N}$ were calculated as indicated under Data Analysis for NanoSIMS Measurements (Section 2.14.3). In order to determine number of TriazNF1 molecules in a specific region of the cell, and implicitly the number of proteins of interest tagged with this probe, the number of TriazNF1 detected by NanoSIMS imaging from each region was calculated using the system of equations shown in :

$$
\begin{gathered}
\text { Number }{ }^{15} \mathrm{~N}+14 * \text { TriazNF1 copy number }={ }^{15} \mathrm{~N} \text { counts } \\
\text { Number }{ }^{14} \mathrm{~N}+16 * \text { TriazNF1 copy number }={ }^{14} \mathrm{~N} \text { counts } \\
\text { Number }{ }^{15} \mathrm{~N} / \mathrm{Number}^{14} \mathrm{~N}=0.00367
\end{gathered}
$$

\section{System of Equations $5 . \quad$ Calculation of TriazNF1 copy numbers}

The copy numbers for TriazNF1 can be inferred from these equations. Here the Number of ${ }^{15} \mathrm{~N}$ and ${ }^{14} \mathrm{~N}$ represent the naturally occurring isotopes in the sample, whereas the measured levels or counts represent the summ of the natural values with the ones introduced by the TriazNF1 probe. The number 0.00367 represents the natural abundance ratio between the main nitrogen isotopes.

By solving the above system of equations, the measured copy number of TriazNF1 is then equivalent to

$$
\text { TriazNF1 copy number }=\frac{{ }^{10} \mathrm{~N} \text { counts }-{ }^{14} \mathrm{~N} \text { counts } * 0.00367}{14-0.00367 * 16}
$$

\section{Equation 6. Derived TriazNF1 copy number formula}

The formula presented in Equation 6 is not an absolute number, but represents only the measured of perceived number of TriazNF1 copies detected in the sample. In order to take into account the number of nitrogen isotopes that were sputtered but failed to ionize and reach the detectors, calibration with standard probes for ${ }^{14} \mathrm{~N}$ and ${ }^{15} \mathrm{~N}$ is required. Based on this calibration, the absolute number of TriazNF1 (and implicitly protein of interest) copies can be determined. 


\subsection{Statistical analysis}

In this study, all barplots are given as mean values \pm standard error of the mean (SEM). For all statistical analyses, the unpaired t-test was calculated using either the built-in function in SigmaPlot (Systat Software Inc.) or the statistical package in Matlab (The Mathworks Inc.). Each figure legend features the $\mathrm{P}$ value ranges, which are as follows: * $\mathrm{P}<0.05, \mathrm{P}<0.01 * *$, and $\mathrm{P}<0.001 * * *$. In the main text $\mathrm{P}$ values below $0.001(* * *)$ are refered to as highly significant. 


\section{RESULTS}

In this study, I took advantage of the smaller size of non-canonical amino acids (ncAAs) to specifically label proteins for different imaging techniques. First, I generated the Amber stop codon constructs for the incorporation of ncAAs in more than 20 proteins (Section 3.1). Then I the tested and optimized the ncAA incorporation and click labeling reaction with different fluorescent dyes (Section 3.2-3.4). Using this optimized protein labeling technique I set out to investigate whether the larger fluorescent protein tags affect the macromolecular arrangements formed by proteins (Section 3.5). In addition, I also employed the genetic encoding of ncAA in conjuction with clickable NanoSIMS probes for specific protein isotopic and fluorescence labeling (SPILL; Section 3.6)

\subsection{Constructs for Non-Canonical Amino Acid Encoding}

Since the site-directed mutagenesis and cloning project was complex, involving the generation of constructs for more than 20 proteins, I included below the technical approach I have taken to obtain these constructs.

\subsubsection{Constructs Obtained by Site-Directed Mutagenesis}

The genetic encoding of ncAA involves the introduction into the target cell of a bioorthogonal aminoacyl-tRNA synthetase and suppressor tRNA (RS/tRNA) pair that direct the incorporation of the ncAA at Amber stop codon positions (Liu and Schultz, 2010). In practice, this is realized by transfecting the cells with: i) a plasmid containing the protein of interest with an in-frame Amber stop codon and ii) another plasmid containing the bioorthogonal RS/tRNA pair. The ncAA has to be exogenously provided (i.e. added to cell medium). Even in transfected cells the ncAA incorporation does not occur with $100 \%$ yield and it depends on many factors: the position of the Amber stop codon in the mRNA, the amount of RS/tRNA expressed in the cell, as well as on the ncAA itself. When the Amber codon is not suppressed but is instead recognized by the endogenous eukaryotic release 
factor eRF-1 (also known as eukaryotic translation termination factor 1, ETF1; Frolova et al., 1994), the nascent polypeptide chain is released at the Amber stop codon position as a truncated version of the full-length protein.

Therefore, the Amber mutants for a protein of interest should be tested and selected. The best paradigm for this is to have an in-frame C-terminal GFP (or any other fluorescent protein) as a reporter for ncAA incorporation. If the ncAA is incorporated (the Amber codon is suppressed), the GFP should be expressed in the transfected cells. When the Amber codon acts as a stop (i.e. no suppression occurs), GFP is not produced. Thus, the GFP levels detected for the different mutants are a readout for ncAA incorporation efficiency.

I started the project with four model proteins: SNAP-25 (25-kDa synaptosomal-associated protein), syntaxin 1, $\alpha$-synuclein, and VAMP2, whose coding sequences I modified to introduce Amber stop codons (see Figure 3-1). I performed site-directed mutagenesis on Cterminal GFP or YFP chimeras of these proteins (see Table 2-7 for the specific primers used). The syntaxin 1 and $\alpha$-synuclein genes were cloned in the plasmids pEYFP-N1 and pEGFP-N3, respectively. For the rest of the genes of interest the pEGFP-N1 backbaone was used as a destination vector (all three vectors were produced by Clontech Laboratories Inc.). Both the pEGFP-N1 and N3 vectors encode for a red-shifted mutant of EGFP containing two substitutions (Phe-64 to Leu and Ser-65 to Thr; Cormack et al., 1996). In contrast, the pEYFP-N1 vector contains yellow-green variant of EGFP with four amino acid substitutions (Ormö et al., 1996).

For each of these proteins, I obtained different Amber stop codon mutants. I tested these mutants for ncAA incorporation using Western blotting or fluorescence microscopy (data not shown) and selected the best ones for further investigations. The best performing mutants for ncAA incorporation are schematically depicted in Figure 3-1. In the case of SNAP-25, the candidate of choice was the Amber (TAG) mutant in which the Phe-84 (F84TAG, Figure 3-1 A) was mutagenized due to its high incorporation efficiency. In addition, the truncated form of this construct does not reach the plasma membrane because the palmitoylation domain of SNAP-25, essential for the membrane attachment of this protein, is located immediately after the phenylalanine-84 residue. The mutant M219TAG also performed well in incorporation tests. However, I used it only for preliminary click reaction trials because the non-labeled truncated forms would contain full-length SNAP-25 (plus a 12-residue linker). This side-product may reach the plasma membrane and affect the measurements of the cluster sizes. 


\section{Results}

A

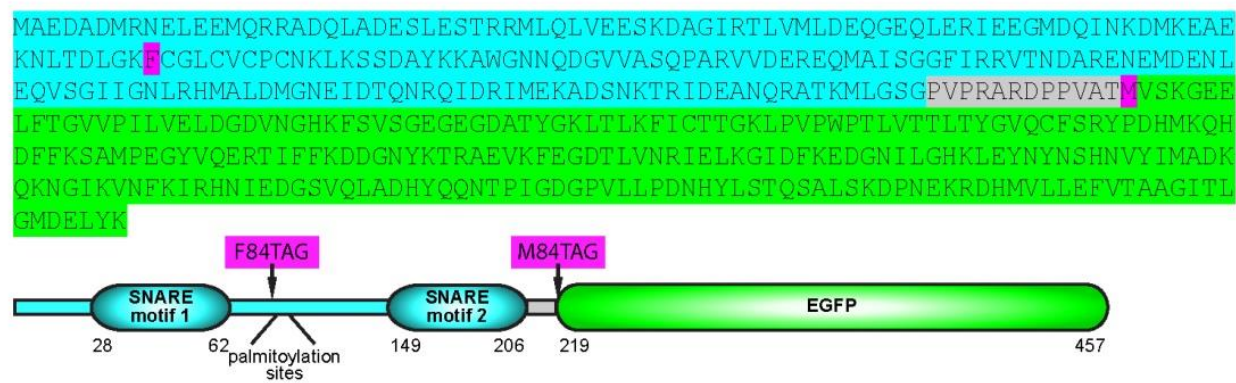

MKDRTQELRTAKDSDDDDDVTVTVDRDRFMDEFEEQVEEIRGEIDK IAENVEEVKRKHSAILASPNPDEKTKEEI EELMSD IKKT ANKVRSKLKSI EQSI EQEE GLNRSSADLRIRKTQHST LSRKFVEVMSEYNAT QSDYRERCKGRIO RQLEITGRTTTSEELEDMLESGN PAIFASGI IMD SS ISKQALSE IET RHSEI I KLEN S I RELHDMFMDMAMLVES QGEMIDRI EYNVEHAVDYVERAVSDTKKAVKYQSKARRKKIMI I I CCVI LGI I IAST I GGI FGLVPRARD PPVAT MVSKGEELFT GVVP I LVELDGDVNGHKFSVSGEGEGDATYGKLTLKF ICTT GKLPVPWPT LVTT FGYGLQCEARY PDHMKQHDFEKSAMP EGYVQERT IFEKDDGNYKTRAEVKFEGDTLVNRIELKGIDFKEDGNI LGHKLEYNYNSHN VY IMADKQKNGI KVNEKIRHN IEDGSVQLADHYQQNTP IGDGPVLLPDNHYLSYQSALSKD PNEKRD HMVLLEFV TAAGITLGMDELYK
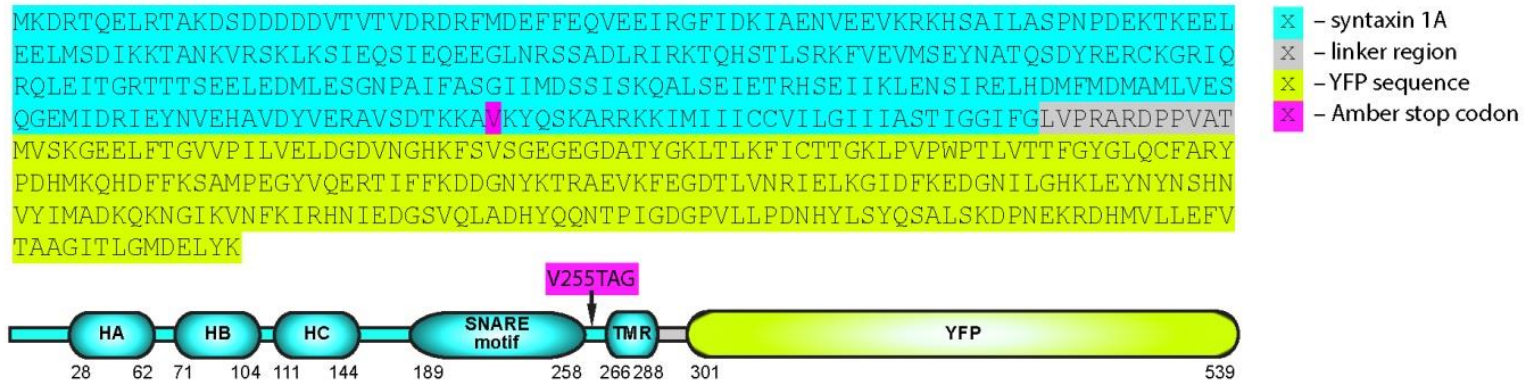

C
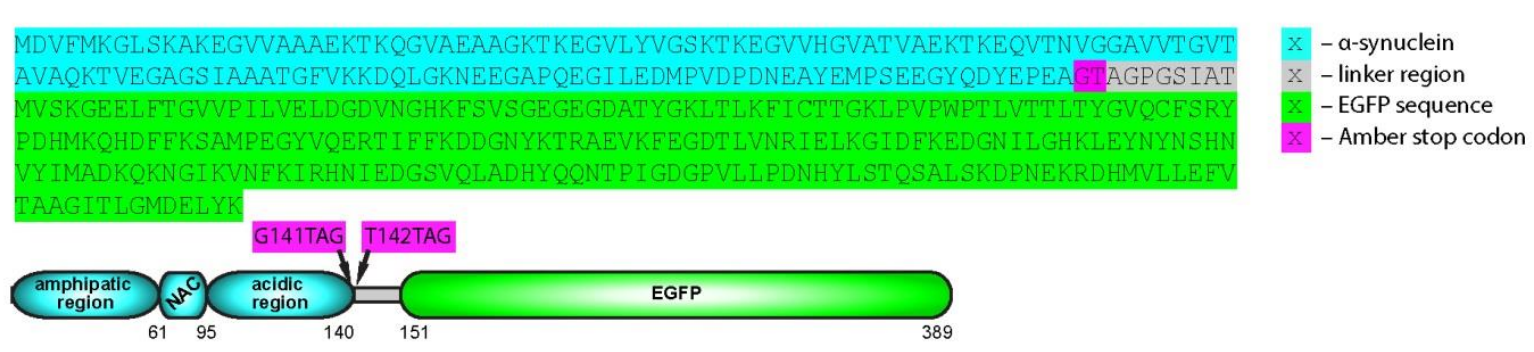

D
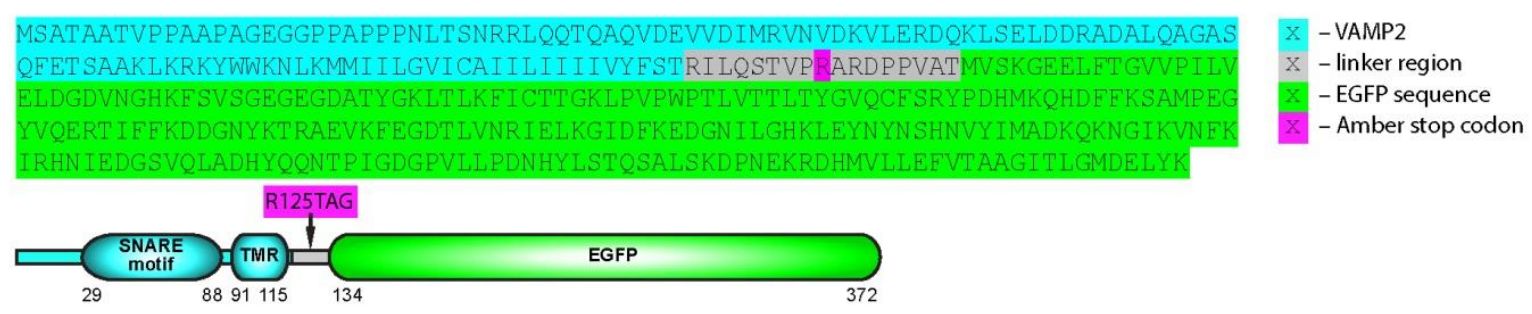

Figure 3-1 Constructs containing fluorescent protein (FP) chimeras with Amber stop codons introduced by site-directed mutagenesis

The amino acid sequences and schematic depictions for SNAP-25 (A), syntaxin 1 (B), $\alpha$-synuclein (C), and VAMP2 (D) constructs are shown. The proteins of interest are depicted in light blue, the fluorescent protein tags are green (for GFP) or yellow (for YFP), and the linker regions are grey, while the amino acid substitutions are marked in magenta. Note that mutant M219TAG for SNAP-25 has been used only in some preliminary experiments. Abbreviations: NAC - non-amyloid- $\beta$ component; SNARE - soluble NSF attachment protein receptor; GFP/YFP - green/yellow fluorescent protein; TMR - transmembrane region. 
A similar approach was used for syntaxin 1, a SNARE protein with one transmembrane domain. In this case, the mutant of choice was V255TAG, in which Val-255 in the short linker between the SNARE domain and the transmembrane region (TMR) was replaced by the Amber stop codon (see Figure 3-1 B). This V255TAG mutation is present before the transmembrane domain of syntaxin 1 and, therefore, the truncated versions do not reach the plasma membrane.

$\alpha$-Synuclein poses more specific requirements because it is not only a cytosolic protein, but it has also been shown to exhibit an altered aggregation behavior if point mutations are introduced in its sequence (especially in its $\mathrm{N}$-terminal amphipathic repeat region; Conway et al., 1998; Conway et al., 2000; Greenbaum et al., 2005; Plotegher et al., 2014). It has also been shown that hydrophobic interactions between the centrally located non-amyloid- $\beta$ component (NAC) regions, more specifically the 12-aa stretch between residues 71 and 82 , are the main driving force behind $\alpha$-synuclein fibrillization (Giasson et al., 2001). The Cterminal truncated forms have been shown to be toxic and promote $\alpha$-synuclein fibrillization (Du et al., 2003). In order to avoid these issues, I decided to insert the Amber stop codons after the full-length coding sequence of $\alpha$-synuclein. Two mutants, G141TAG and T142TAG (Figure 3-1 C), underwent incorporation with the highest efficiency (data not shown) and I employed them in all subsequent experiments.

Vesicle-associated membrane protein 2 (VAMP2), also known as synaptobrevin, has its own particular requirements. I initially intended to use it for live endocytosis experiments. For this, the labeling conditions required that the ncAA be present on the surface of the plasma membrane. However, VAMP2 has only a short transmembrane domain that does not reach the cell surface (Stein et al., 2009). Therefore, I designed several mutations in the linker domain between VAMP2 and GFP: R117TAG, I118TAG, S121TAG, and R125TAG. I tested the incorporation efficiency into the four different mutants of two different ncAAs: AZK and PRK. AZK seems to be incorporated with somewhat higher efficiency than PRK in all the mutants, as can be observed in both VAMP2 and GFP immunoblots (Figure 3-2 A-B). Further confirmation was provided by the GFP fluorescence intensity in cells (see Figure $3-8)$. 


\section{Results}
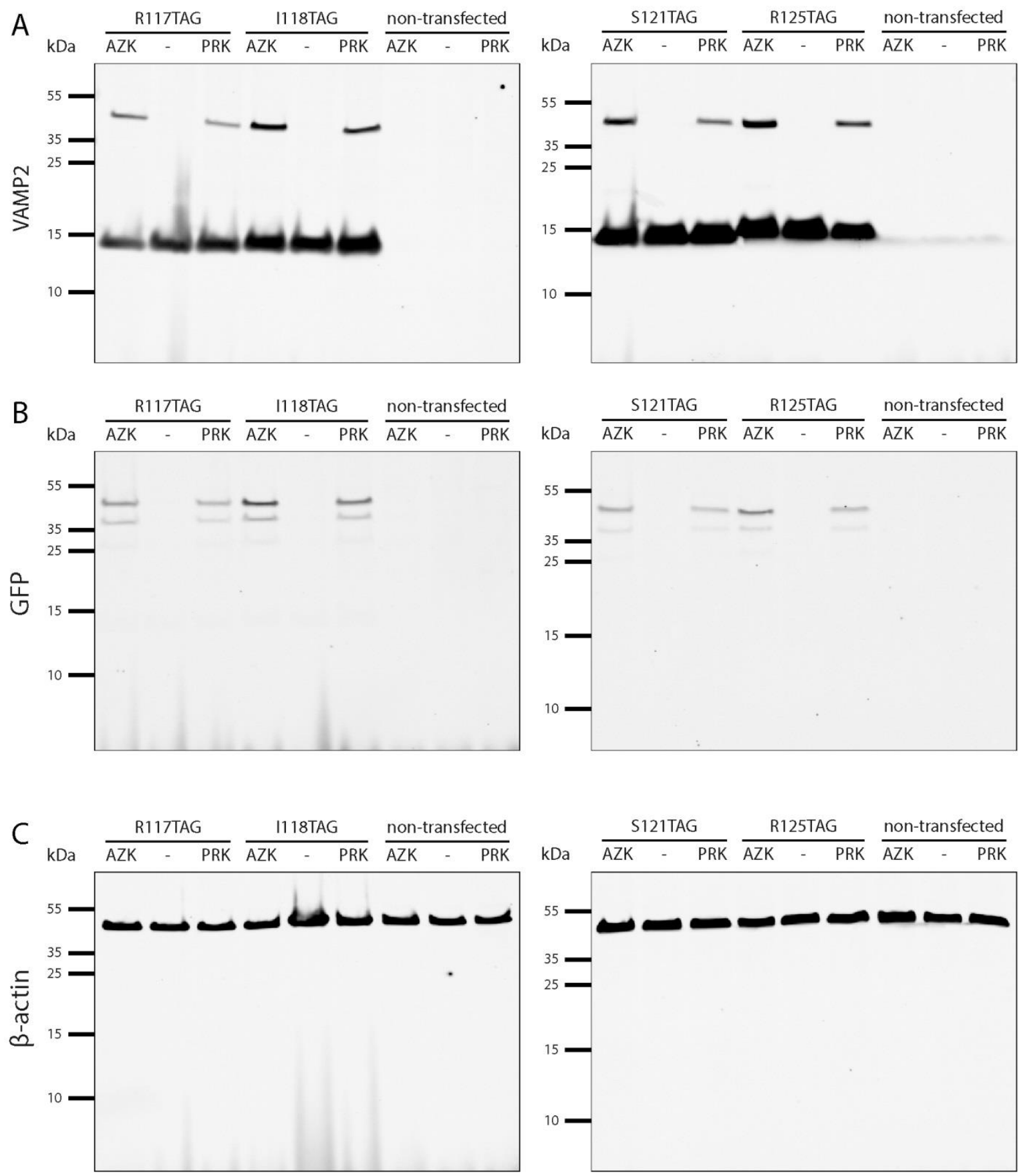

\section{Figure 3-2 Testing ncAA incorporation using Western blotting}

BHK cells have been allowed to incorporate azido-L-lysine (AZK) or propargyl- L-lysine (PRK) or no amino acid (-) for 18 hours while expressing one of the four different VAMP2 mutants: R117TAG, I118TAG, S121TAG, R125TAG. The non-transfected cells are used in this case as controls. Note that no VAMP2 is expressed endogenously in BHK cells. For the transfected cells, the samples where no ncAA was added served as negative controls. The blots were probed with anti-VAMP2 antibodies (A), anti-GFP (B), and anti- $\beta$-actin $(\mathbf{C})$. Panels on the left and right are imaged using the same parameters and the images are identically scaled. The position of the marker lines are indicated on the left-side of each blot in kilodaltons ( $\mathrm{kDa}$ ). VAMP2 runs as a band with the apparent molecular weight (MW) of $12.8 \mathrm{kDa}$, while VAMP2-GFP should be detected at around $41.5 \mathrm{kDa}$. The $\beta$-actin staining was used as a control for sample loading. 
The C-terminal GFP acted as a reporter for ncAA incorporation efficiency: if the ncAA is well incorporated, the VAMP2-GFP band should be more intense (see Figure 3-2, panels A and B). The large bands observed in Figure 3-2 A represent truncated versions generated when the Amber codon acts as a stop. Note that in the control samples where no ncAA was added, there is no detectable VAMP2-GFP band. The $\beta$-actin staining (Figure 3-2 C) indicates approximately equal gel loading for each of the samples. Based on these blotting results, the best mutant out the four tested for VAMP2 is the R125TAG one (see Figure 3$1 \mathrm{D})$. So I selected the R125TAG mutant for further investigations of VAMP2 clustering. What is more, I used VAMP2-GFP R125 backbone to clone other proteins, as described below (Section 3.1.2).

The above-mentioned constructs were all obtained initially as chimeric versions of the proteins of interest, either having a GFP tag (in the case of SNAP-25, $\alpha$-synuclein, and VAMP2) or a YFP one (for syntaxin 1) at the C-terminus. The main reason for this was to have the GFP/YFP fluorescence or immunoblot detection as a reporter for the efficiency of ncAA incorporation for each construct. After the best constructs were selected, I excised enzymatically the fluorescent protein (FP) moiety while retaining the full length coding sequence for the protein of interest. Refer to Figure 3-3 for details regarding the constructs without FP tags.

In this study, I have also included mutants obtained through site-directed mutagenesis by other researchers (see Materials and Methods, Section 2.2 for details): mutant K676TAG for insulin receptor (InsR; Nikić et al. 2014) and K101TAG for 5HT1a (Dr. Marcus Niebert). 


\section{Results}

A

MAEDADMRNELEEMQRRADQLADESLESTRRMLQLVEESKDAGIRTLVMLDEQGEQLERIEEGMDQINKDMKEA KNLT DLGKFCGLCVCPCNKLKSSDAYKKAWGNNQDGVVASQPARVVDEREQMAI SGGF I RRVTNDARENEMDENI EQVSGI I GNLRHMALDMGNEIDTQNRQIDRIMEKADSNKTRIDEANQRATKMLGSG

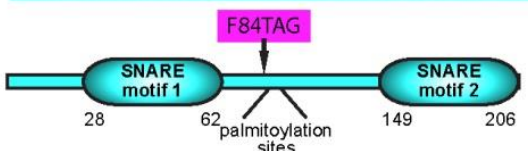

- SNAP-25B sequence

- Amber stop codon substitution

B

MKDRT OELRTAKDSDDDDDVTVTVDRDREMDEFEEOVEE IRGE IDKIAENVEEVKRKHSAI LASPNPDEKTKEEI EELMSDI KKTANKVRSKLKS IEQS IEQEEGLNRSSADLRIRKTQHSTLSRKFVEVMSEYNATQSDYRERCKGRIQ RQLEITGRTTT SEELEDMLESGNPAI FASGI IMDSS I SKOALSEI ETRHSEI IKLENS IRELHDMFMDMAMLVES QGEMIDRIEYNVEHAVDYVERAVSDTKKAVKYQSKARRKKIMI I ICCVILGI I IASTI IGGIFG

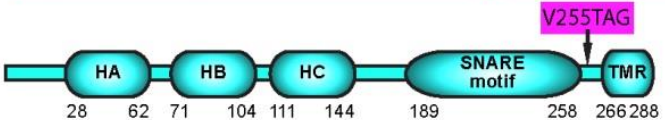

- syntaxin 1 A sequence

- Amber stop codon substitution

C

MDVEMKGLSKAKEGVVAAAEKTKQGVAEAAGKTKEGVLYVGSKTKEGVVHGVATVAEKTKEQVTNVGGAVVTGVTI AVAQKTVEGAGS IAAATGFVKKDQLGKNEEGAPQEGILEDMPVDPDNEAYEMP SEEGYQDYEPEAC

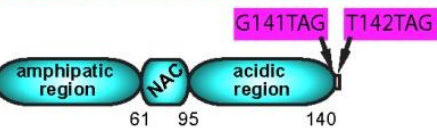

$\mathrm{x}$ - a-synuclein sequence

- Amber stop codon substitution

D

MSATAATVPPAAPAGEGGPPAPPPNLT SNRRLQQT QAQVDEVVD IMRVNVDKVLERDQKLSELDDRADALQAGAS QFETSAAKLKRKYWWKNLKMMI I LGVICAI I L I I IVYESTRILQSTVP

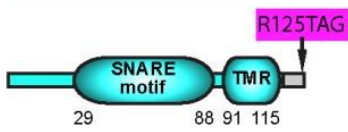

X - VAMP2 sequence
$X$ - linker region
X - Amber stop codon substitution

\section{Figure 3-3 Constructs without fluorescent protein (FP) tags}

Light blue, greyand magenta colors were used to highlight the amino acid sequences, the linker regions, and the Amber stop codon substitutions, respectively. The sequences and schematic depictions are shown for SNAP-25 (A), syntaxin 1 (B), $\alpha$-synuclein (C), and VAMP2 (D). Abbreviations: NAC - non-amyloid- $\beta$ component; SNARE - soluble NSF attachment protein receptor; GFP/YFP - green/yellow fluorescent protein; TMR - transmembrane region. 


\subsubsection{Constructs Obtained by Cloning}

In a second phase of the project, I expanded the study to 20 more proteins. The strategy for this cloning project involved the use of pEGFP-N1 VAMP2-GFP R125TAG (+GFP) and pN1 VAMP2 R125TAG A126TAA (-GFP) as destination vectors (see Figure 3-4). I excised the VAMP2 coding sequence from both of these vectors and in its place I inserted the coding sequences for the 20 new proteins. Please refer to Section 2.2.2 for a detailed description of the primers, restrictions enzymes, and protocols employed to perform the clonings; Table 2-6 for a list of the proteins included in this study, and the annex for the schematic depictions of the construct sequences. For each of these additional 20 proteins (see Table 2-8), I generated two constructs with Amber stop codons: one with a GFP tag and one without the tag. The genetic encoding of PRK into these constructs will be discussed in Section 3.4 .

Therefore, almost all (except syntaxin 1) of the fluorescent protein chimeras used in this study contain the GFP mutant 1 (Phe-64 to Leu and Ser-65 to Thr) obtained by Cormack and collaborators (Cormack et al., 1996). This variant is brighter and more red-shifted (excitation maximum $=488 \mathrm{~nm}$; emission maximum $=507 \mathrm{~nm}$ ) as well as optimized for expression in mammalian cells. This is because the GFP sequence has been engineered with silent base changes to comply to human codon usage preferences (Haas et al., 1996) and its upstream flanking region has been modified to encompass a Kozak translation initiation site (Kozak, 1987). The C-terminal GFP chimeras are not only excellent reporters for ncAAs incorporation as described above, but this orientation is also beneficial for retaining the fluorescent properties especially for in vivo localization studies (Tajima et al., 2010). 
A

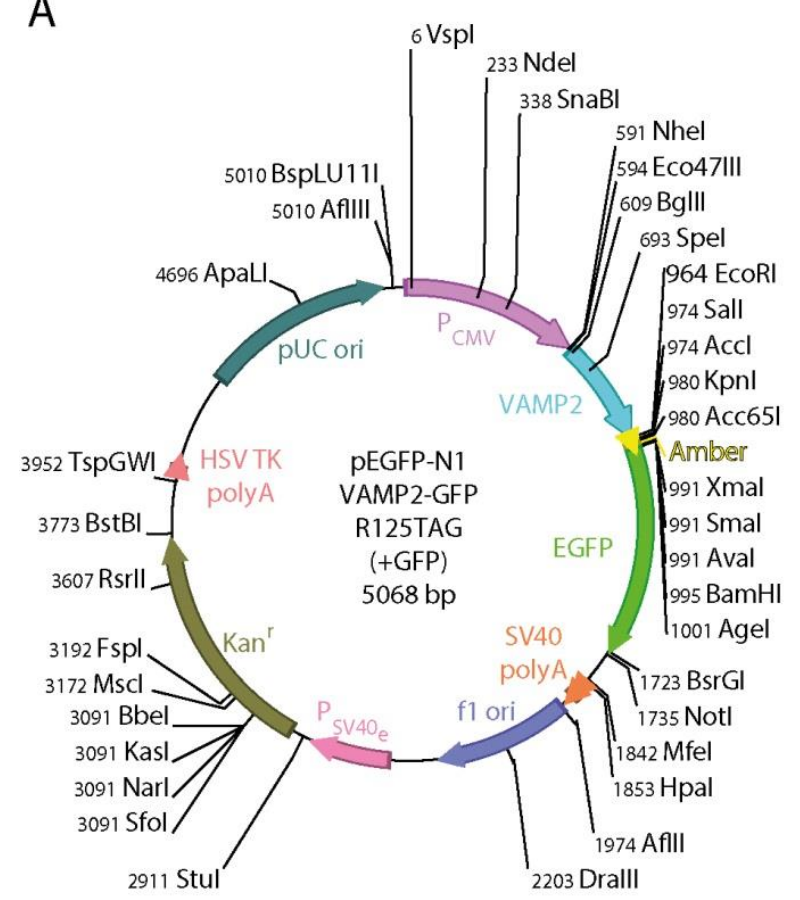

B

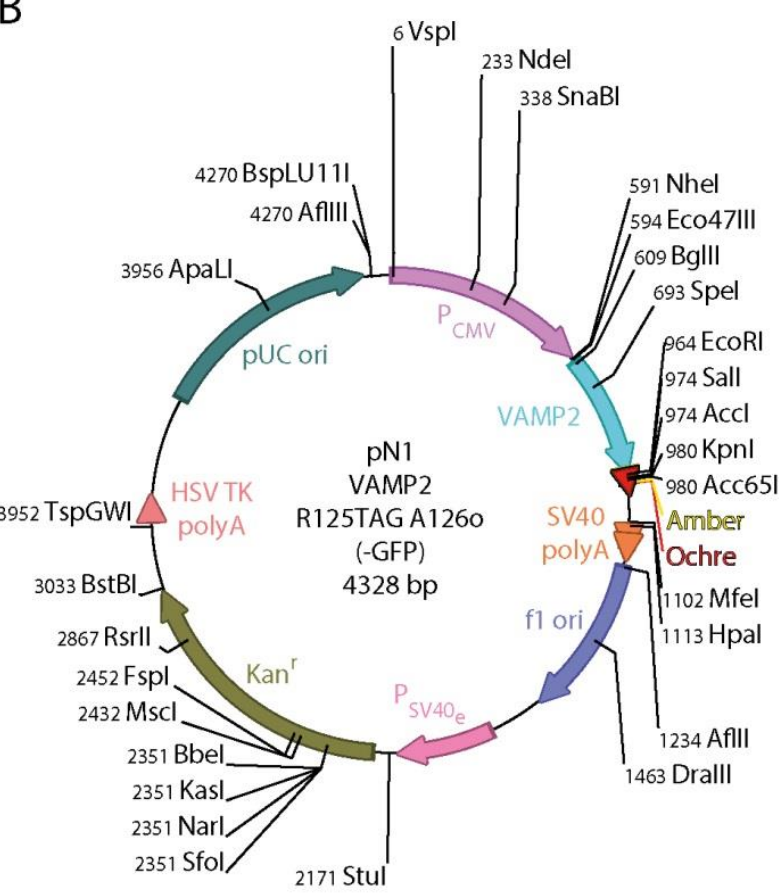

\section{Figure 3-4 Restriction maps for the VAMP2 pEGFP-N1 and pN1 vectors}

The pEGFP-N1 (A) and the pN1 (B) vectors contain the VAMP2 gene (shown in light blue) and an Amber stop codon (depicted in yellow). Aside from this, the pN1 vector lacks the EGFP coding sequence (removed with the SmaI and NotI restriction enzymes) but has an additional Ochre stop codon (in red) immediately after the Amber one. The backbones of these two vectors are identical and include the following components for proper replication, and transcription in mammalian cells: the human cytomegalovirus promoter $\left(\mathrm{P}_{\mathrm{CMV}}\right.$, in purple), the Simian virus 40 mRNA polyadenylation signals (SV40 polyA, in orange), an SV40 early promoter $\left(\mathrm{P}_{\mathrm{SV} 40 \mathrm{e}}\right.$, in pink), the transposon Tn5 gene for kanamycin/neomycin resistance $\left(\mathrm{Kan}^{\mathrm{R}} / \mathrm{Neo}^{\mathrm{R}}\right.$; in olive color), and the Herpes simplex virus thymidine kinase polyadenylation signals (HSV TK polyA; in peach color). As optional features, the SV40 origin of replication confers these constructs the possibility to be replicated in cell lines expressing the SV40T antigen ( $\mathrm{P}_{\mathrm{SV} 40 \mathrm{e}}$, in pink), while the cassette containing the $\mathrm{P}_{\mathrm{SV} 40 \mathrm{e}}-\mathrm{Neo}^{\mathrm{R}}-\mathrm{HSV}$ TK polyA allows the generation of stably transfected lines using G418 selection. Another promoter upsteam of the above-mentioned cassette is responsible for the transcription of the kanamycin resistance gene for amplification in bacterial cell lines. Other sequences of note for propagation in bacteria include the pUC origin of replication (pUC ori; in turquoise), and the $\mathrm{f1}$ phage origin of replication (f1 ori; in dark purple) that enables the production of single stranded DNA and packaging into viral particles. Both depictions contain the unique restriction sites and the names of the enzymes that can be used. 


\subsection{Testing and Optimizing Different Clickable ncAAs}

As a first step in establishing a system for labeling proteins via ncAA incorporation and click labeling, I tested different amino acids previously described to undergo copper-mediated (Figure 3-5 A) or a copper-free click reaction (Figure 3-5 B).

A<smiles>N#CCOC(=O)NCCCC[C@H](N)C(=O)O</smiles>

AZK

B<smiles>NC(CCCCNC(=O)OCC1[C@H]2CCC#CCC[C@H]12)C(=O)O</smiles>

$\mathrm{BCNK}$ racemate<smiles>Nc1ccc(C[C@H](N)C(=O)O)cc1</smiles>

pAzpa<smiles>NC(CCCCNC(=O)OC1C#CCCCCC1)C(=O)O</smiles><smiles>C#CCOC(=O)NCCCC[C@H](N)C(=O)O</smiles>

PRK<smiles>NC(CCCCNC(=O)OC1C=CCCCCC1)C(=O)O</smiles>

TCOK

Figure 3-5 The structures for the ncAAs tested in this study

A. Chemical formulas of the non-canonical amino acids that undergo copper(I)-catalyzed azide-alkyne cycloaddition (CuAAC): lysine derivatives azido-L-lysine (AZK) and propargyl-L-lysine (PRK), and phenylalanine derivative $p$-azido-L-phenylalanine (pAzpa). B. These lysine derivatives are amenable for copper-free click chemistry: bicyclo[6.1.0]nonyne-L-lysine (BCNK) and strained cyclooct-2-yneL-lysine (SCO) can undergo both strain promoted azide alkyne cycloaddition (SPAAC) and strainpromoted inverse-electron-demand Diels-Alder cycloaddition (SPIEDAC), while trans-cyclooct-2ene-L-lysine (TCOK) reacts only in SPIEDAC. Note that the BCNK compound used in this study was a racemic mixture. 


\section{Results}

Another goal of this study was to optimize the click chemistry labeling reaction with fluorescent dyes that are suitable for super-resolution investigations. For this, I have tested three organic fluorophores that emit in the deep red region of the spectrum (their chemical formulas and 3D renderings can be seen in Figure 3-6).

For GSDIM microscopy (Fölling et al., 2008), I tested the indocarbocyanine synthetic dye Alexa647 (Figure 3-6 A), which has been widely used in stochastic optical reconstruction miscroscopy (STORM) due to its good blinking properties. Alexa647 contains four sulfonate groups (compared with only two for Cy5), which improves the solubility of this dye in water. In contrast, other indocarbocyanines, such as $\mathrm{Cy} 3$ and $\mathrm{Cy} 5$ that have also been used for pointillistic super-resolution measurements are more hydrophobic. $\mathrm{Cy} 3$ and $\mathrm{Cy} 5$ have a strong tendency to multimerize or to form aggregates in aqueous solution and upon their interaction with biomolecules (Levitus and Ranjit, 2011). So I selected the Alexa647 as a fluorophore of choice for GSDIM imaging.

The carbopyronine dye Atto647N (see Figure 3-6 B) was one of the first dyes used in STED microscopy. The Atto647N molecule is photostable and exhibits a high fluorescence quantum yield. These properties enable the Atto647N fluorophore to withstand the high laser intensities required for the depletion to take place and multiple rounds "ON-OFF" switching (Kolmakov et al., 2010; Wurm et al., 2012; Stennett et al., 2014).

The sulfonated rhodamine derivative KK114, and its commercial phosphorylated variant Star635P, represent a new class of red-shifted fluorophores with very high fluorescence quantum yields and stability. For the published chemical structures see Figure 3-6 C. These dyes were optimized to be hydrophilic and to minimize their unspecific binding to membranes or other cellular material, leading to an excellent signal to noise ratio when used on biological samples (Kolmakov et al., 2010; Wurm et al., 2012). KK114 has been successfully applied in confocal and STED microscopy (Hua et al., 2011; Wurm et al., 2011). 
A

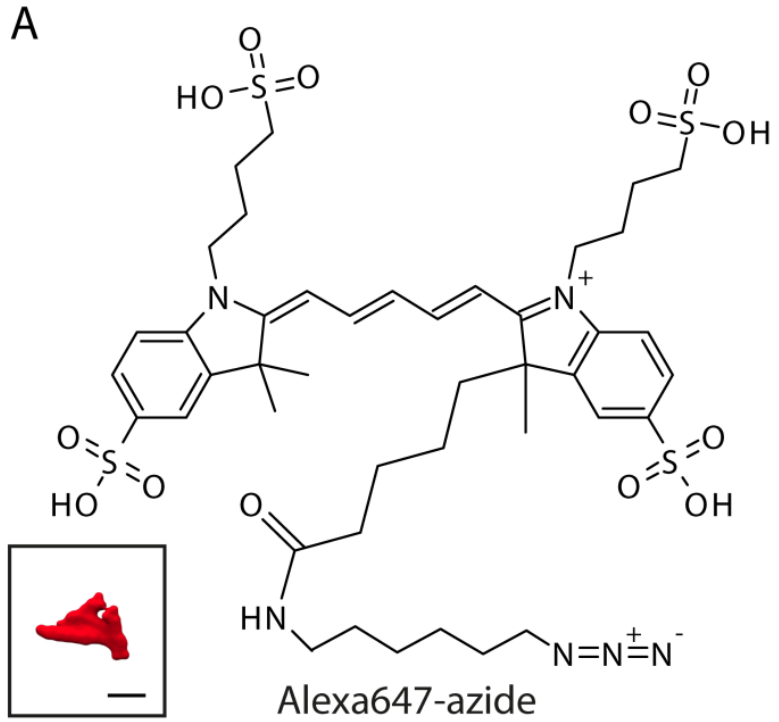

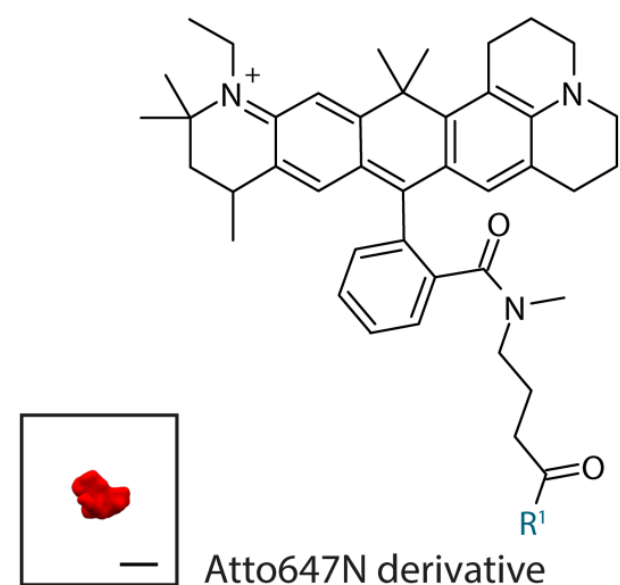

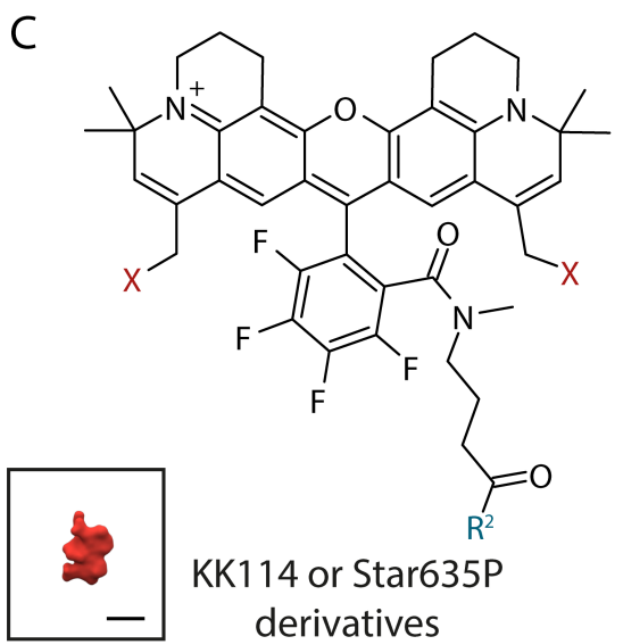<smiles>[N-]=[N+]=NCCNCc1ccc(-c2nncnn2)cc1</smiles>

$\mathrm{X}=\mathrm{SO}_{3} \mathrm{H}(\mathrm{KK} 114)$ or $\mathrm{OPO}_{3} \mathrm{H}_{2}(\mathrm{Star635P})$

Figure 3-6 Structures for the fluorophores used in this project and putative other dyes.

The chemical structures for the Alexa647N (A), Atto647N (B), and KK114 and Star635P fluorophores are depicted. Note that the $\mathrm{R}^{1}$ moiety in the case of the azide derivative of Atto647N is uncertain (based on the molecular weight the linker region should be longer than the one shown in the figure), as this is not disclosed by the producer of this compound. The insets contain 3D chemical structures of the corresponding dyes. Scale bar for insets, $1 \mathrm{~nm}$. 


\subsection{1 ncAAs for Copper-Catalyzed Click Reaction}

I tested three different ncAAs amenable to copper(I)-catalyzed alkyne-azide cycloaddition (CuAAC): two azide derivatives of phenylalanine and lysine, pAzpa and AZK, respectively, as well as the alkyne derivative of lysine, PRK. The chemical formulas of these compounds are shown in Figure 3-5.

pAzpa (p-azido-L-phenylalanine)

The non-canonical amino acid pAzpa has been added to the genetic code of Escherichia coli using the Methanococcus jannaschii tyrosyl-tRNA synthetase and a mutant tyrosine amber suppressor tRNA (Chin et al., 2002). It has also been used in Saccharomyces cerevisiae with the help of the tyrosyl-tRNA synthetase and Amber suppressor tRNA from E. coli (Chin et al., 2003). The incorporation of $p$-azido-L-phenylalanine and $p$-(propargyloxy)-Lphenylalanine has been achieved in E.coli using the optimized pEvol system comprising the tyrosyl-tRNA synthetase and tRNA pair from Methanocaldococcus janaschii (Young et al., 2010).

pAzpa has been among the first clickable ncAAs incorporated in mammalian cells (Liu et al., 2007) using a mutant E. coli aaRS and a Bacillus stearothermophilus suppressor tRNA. In the same paper, a propargyloxy (alkyne) derivative of phenylalanine was also employed but its incorporation efficiency was lower than for pAzpa. This compound is not commercially available, so I did not test it for my project.

In this study, I have used the plasmid system pcpAzpaRS described by Yokoyama and collaborators to incorporate pAzpa in mammalian cells. The preliminary results from Figure 3-7 indicate that the incorporation of pAzpa was successful (see the GFP signal which is present only if the Amber stop codon was suppressed). Note that in the control where no ncAA was provided, there is no GFP signal, even though the cells were also transfected under the same conditions as for the pAzpa (Figure 3-7). This attests the fidelity of the incorporation of the ncAA and no other endogenuous amino acid. In terms of the click labeling efficiency, the results indicate nonspecific background in all cells, both in the control and the sample incubated with pAzpa (see Figure 3-7). What is more, the click signal is above background only for the cell with high expression levels SNAP-25-GFP, while the cells with low SNAP-25-GFP levels cannot be distinguished from the rest of the cells. 

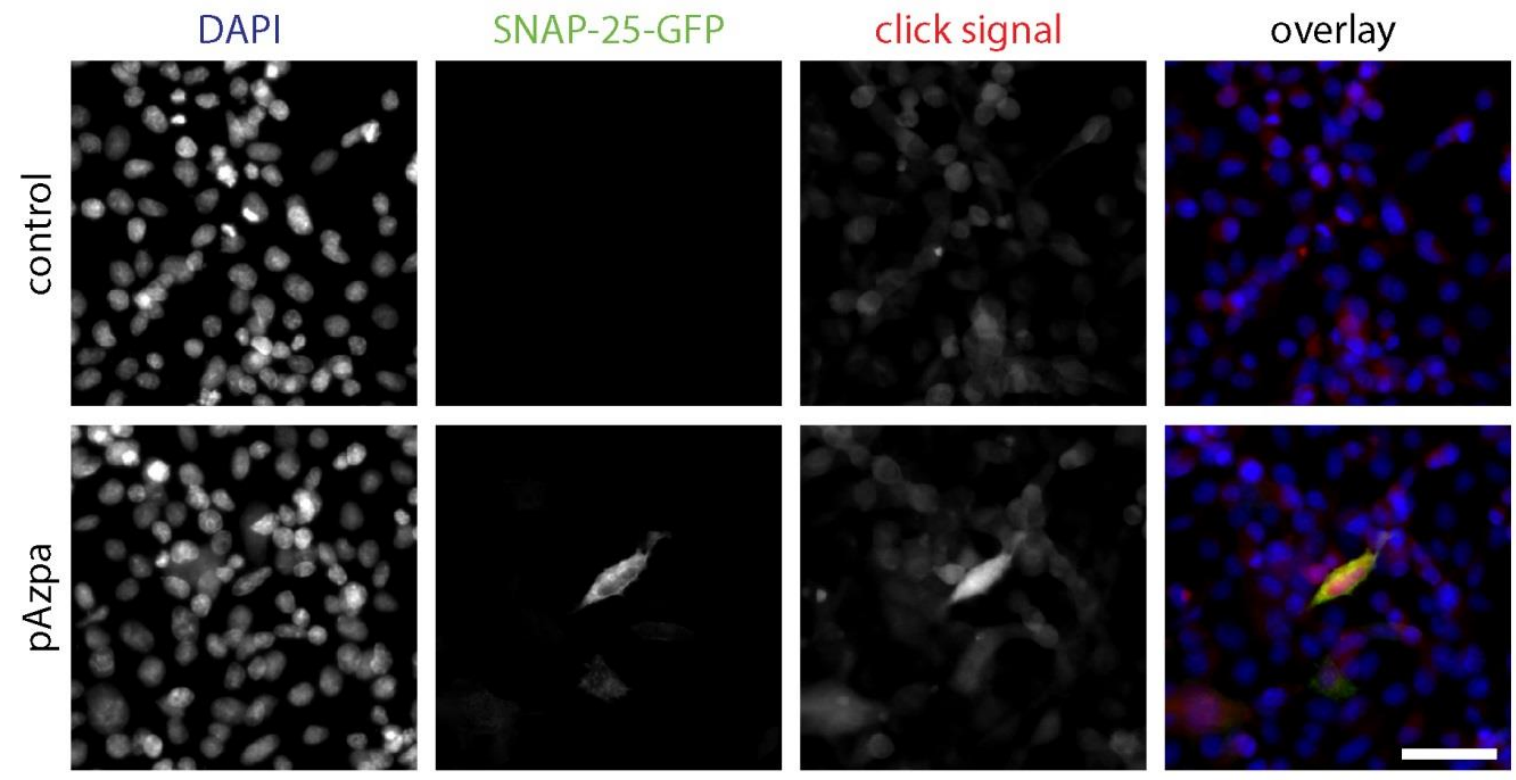

Figure 3-7 pAzpa click reaction results in low signal-to-noise levels

BHK cells were transfected with the pEGFP-N1 SNAP-25-GFP F84TAG and the pcpAzpaRS vectors. The ncAA pAzpa (lower panel) was provided in the cell medium for approximately 18 hours, while the cells were allowed to express the protein of interest. In the control, no ncAA was added even though the cells were cotransfected under the same conditions. Before fixation, the cells were incubated for 2 hours with normal medium to remove the pAzpa excess. The click reaction was carried out with $2 \mu \mathrm{M}$ Atto647N-alkyne and the nuclei were revealed with DAPI. The fluorescence intensity for the DAPI, GFP and click signal in both the pAzpa and the control were identically scaled. In the rightmost panels, overlays of the three different channels can be seen. Scale bar applies to all images and is $40 \mu \mathrm{M}$.

This improper signal-to-noise ratio is a result of the relative lability of the azido group of pAzpa, which delivers low labeling levels, on one hand, and of the hydrophobicity of Atto647N, on the other hand (Kolmakov et al., 2010; Hughes et al., 2014). As a next step, I tried to optimize the click reaction by using other fluorescent dyes that generate a lower background, such as Star635P or Alexa647.

The pAzpa compound has several major disadvantages: it requires a $80 \%$ acetic acid solution to get dissolved as a dark yellow solution (maximum concentration is $0.242 \mathrm{M}$ ) and it is also highly unstable in solution. The saturated pAzpa solution in $80 \%$ acetic acid has to be freshly prepared and used within 5 minutes. Upon longer storage at RT or $-20^{\circ} \mathrm{C}$, it gets degraded and forms an amorphous precipitate. Additionally, pAzpa is a derivative of a bulky and hydrophobic amino acid - phenylalanine - and poses problems when substituting other smaller and more hydrophilic amino acids. If a hydrophobic residue is replaced, then the azido group of pAzpa might be secluded in a hydrophobic pocket and the labeling reaction could be impaired. Therefore, due to the above reasons and especially due to the high 


\section{Results}

instability of the pAzpa, I resorted to finding better ncAA candidates, as can be seen in the rest of this chapter.

\section{AZK (azido-L-lysine) and PRK (propargyl-L-lysine)}

The two aliphatic ncAAs azido-L-lysine (AZK) and propargyl-L-lysine (PRK) have been genetically encoded by bacteria using the Methanosarcina barkeri pyrrolysyl-tRNA and suppressor tRNA ${ }^{\text {Pyl }}$ (Nguyen et al., 2009; Milles et al., 2012). In this study, I used the optimized plasmid pCMV PylRS/tRNA ${ }^{\text {Pyl }}$ WT containing the Methanosarcina mazei bioorthogonal tRNA ${ }^{\text {Pyl }}$ and the wild-type (WT) pyrrolysyl-tRNA synthetase (Plass et al., 2011).

The Western blotting results from Figure 3-2 indicate that both of these ncAAs are well incorporated in VAMP2-GFP but the efficiency of the incorporation is slightly higher for AZK compared with PRK.

As a next step, I wanted to investigate not only the incorporation efficiency, but also the suitability to undergo labeling reactions for these lysine derivatives. For this, I resorted to fluorescence microscopy. I expressed VAMP2-GFP R125TAG and the bioorthogonal RS/tRNA pair in BHK cells in the absence (Figure 3-8 A) or in the presence of ncAAs (Figure 3-8 B-C). I subjected the fixed and permeabilized cells to CuAAC labeling with Atto647N derivatives. As can be seen in Figure 3-8, AZK and PRK are both effectively incorporated. However, only PRK is specific and efficient in the copper-catalyzed click reaction (compare the click signal panels with the VAMP2-GFP panels in Figure 3-8 C).

The reason behind this could be the high sensitivity of the azide group to undergo reduction, resulting in a non-reactive byproduct (Milles et al., 2012). It is plausible that after the incorporation of AZK into the protein of interest (in this case VAMP2-GFP), the clickable azide moiety was inactivated. The lack of reactivity of the azide after the incubation at $37^{\circ} \mathrm{C}$ for approximately one day was further proven by its poor labeling with the strained azadibenzobicyclooctyne (DIBAC) derivative of KK114 (data not shown). The latter reaction was first shown to effectively label enzymes in copper-free cycloaddition (Debets et al., 2010) and was later applied for the in vivo labeling of cells treated with L-azidohomoalanine (Saka et al., 2014a). 

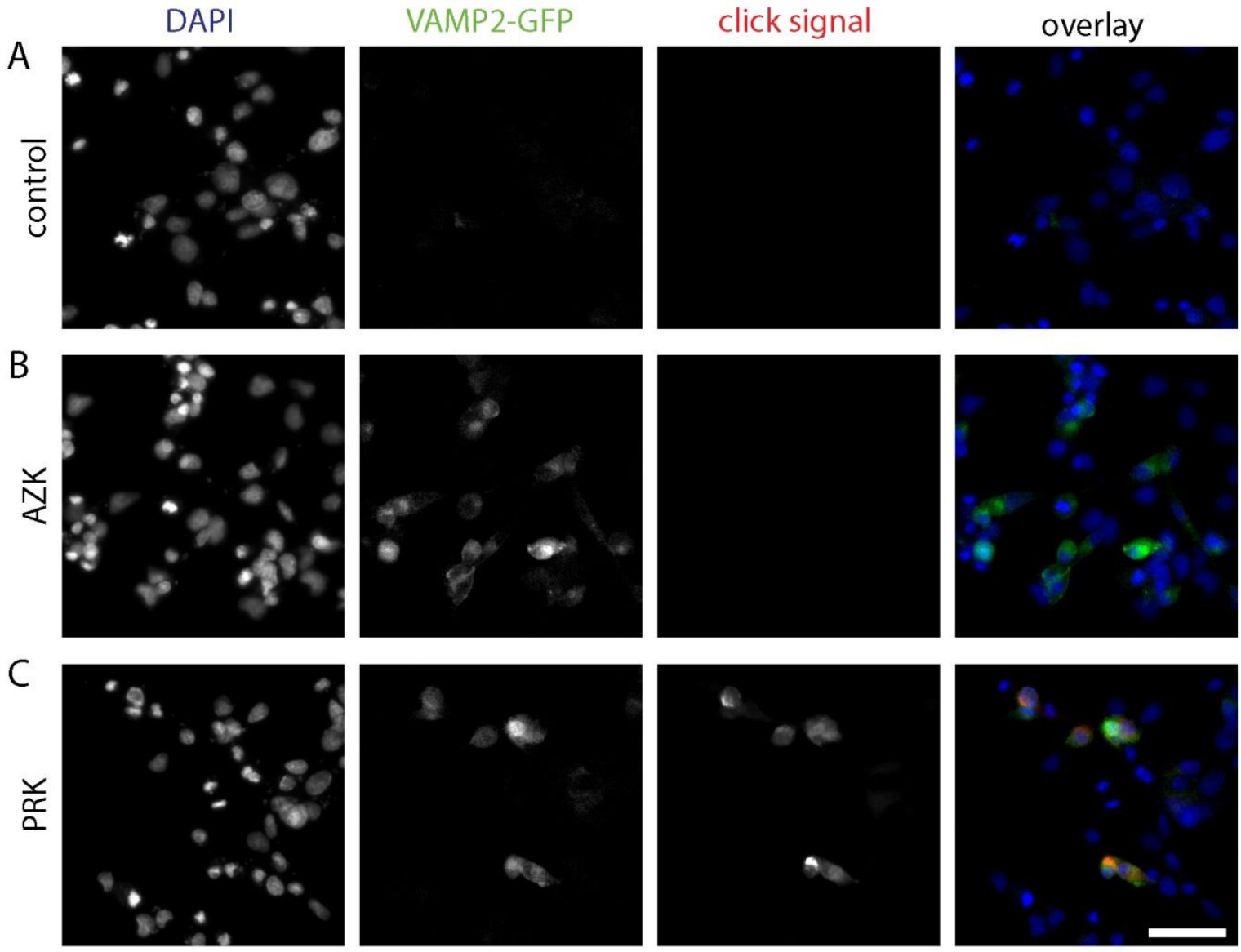

Figure 3-8 AZK and PRK are well incorporated into VAMP2, but only PRK reacts efficiently in CuAAC

VAMP2-GFP R125TAG was expressed in BHK cells in the absence of any ncAA (A) or in the presence of AZK (B) or PRK (C). Approximately 18 hours post-transfection the cells were washed for 2 hours in normal medium, then fixed and permeabilized. Copper-catalyzed click reaction was performed with $2 \mu \mathrm{M}$ Atto647N-alkyne (A-B) or $2 \mu \mathrm{M}$ Atto647N-azide (C). Note that the images for each of the channels were scaled identically. Nuclei were revealed using DAPI staining. Scale bar, $40 \mu \mathrm{m}$.

Therefore, out of the three ncAAs suitable for copper-catalyzed click reaction, only PRK is well incorporated and reacts with the complementary fluorescent dyes in a specific and efficient manner. A summary of the results for the ncAA testing and optimization can be found in Table 3-1. As reported in Section 3.2.3, I sought to further optimize the labeling of PRK samples using more hydrophilic dyes at higher concentrations to ensure that all alkyne groups are saturated during the click labeling reaction. 


\subsection{2 ncAAs for Copper-Free Click Reaction}

For click chemistry in the absence of any metal ion catalyzers, I tested the following ncAAs: strained cyclooct-2-yne-L-lysine (SCOK), bicyclo[6.1.0]nonyne-L-lysine (BCNK), and transcyclooct-2-ene-L-lysine (TCOK). Their chemical structures are shown in Figure 3-5 B.

In contrast to the amino acids for copper-catalyzed click reaction, which contain simple azide or alkyne groups, the amino acids employed in the copper-free click reactions contain highly strained alkyne or trans-alkene groups within rings of more than eight carbons (compare Figure 3-5 A with B). These compound are more reactive if the number of carbons in the ring is higher because this increases the strain on the double or triple bonds (they are more deviated from the normal geometry that they would normally adopt). This leads to an increased reactivity in copper-free click reaction. Therefore, based on the ring strain, it is expected that BCNK and TCOK would be more reactive than SCOK. All these strained lysine derivatives are amenable for copper-free click chemistry as follows: BCNK and SCOK can undergo both strain-promoted azide alkyne cycloaddition (SPAAC) with azide groups and strain-promoted inverse-electron-demand Diels-Alder cycloaddition (SPIEDAC) with tetrazine groups, while TCOK reacts only in SPIEDAC (Nikić et al., 2014).

For the incorporation of these bulky amino acids, wild-type PylRS/tRNA pair which efficiently incorporates the smaller lysine derivatives (e.g. AZK and PRK) cannot be used because the incorporation yield is very low. A mutant version of the PylRS (Tyr-306 to Ala and Tyr-306 to Phe) has been optimized by Yokoyama and collaborators to efficiently incorporate bulky lysine derivatives (Yanagisawa et al., 2008). The plasmid system used in this project was further optimized by Lemke and coworkers (Plass et al., 2012).

\section{SCOK (strained cyclooct-2-yne-L-lysine)}

The first strained ncAA I had the chance to test for incorporation and click efficiency was SCOK (Plass et al., 2012). I first performed copper-free click chemistry with Atto647Nazide, but the results showed considerable background staining and I could not detected any specific signal (data not shown). As the SPAAC reaction requires at least $10 \mu \mathrm{M}$ of azidederivative for the reaction to have high enough yield, I resorted to the more hydrophilic coumarin derivative KK114-azide (see Figure 3-6 C for the chemical structure). 
I therefore performed SCOK incorporation into SNAP-25-GFP (see Figure 3-9) and assessed the specificity of the copper-free click labeling. Note that in Figure 3-9 there is some unspecific staining for the click channel in all cells, both in the control where no ncAA was added and in the conditions where SCOK has been incorporated in SNAP-25-GFP. The specificity of SCOK incorporation is proven by the specific GFP fluorescence arising in transfected cells fed with the ncAA. As the GFP is located at the C-terminus, it acts as a reporter of ncAA incorporation.
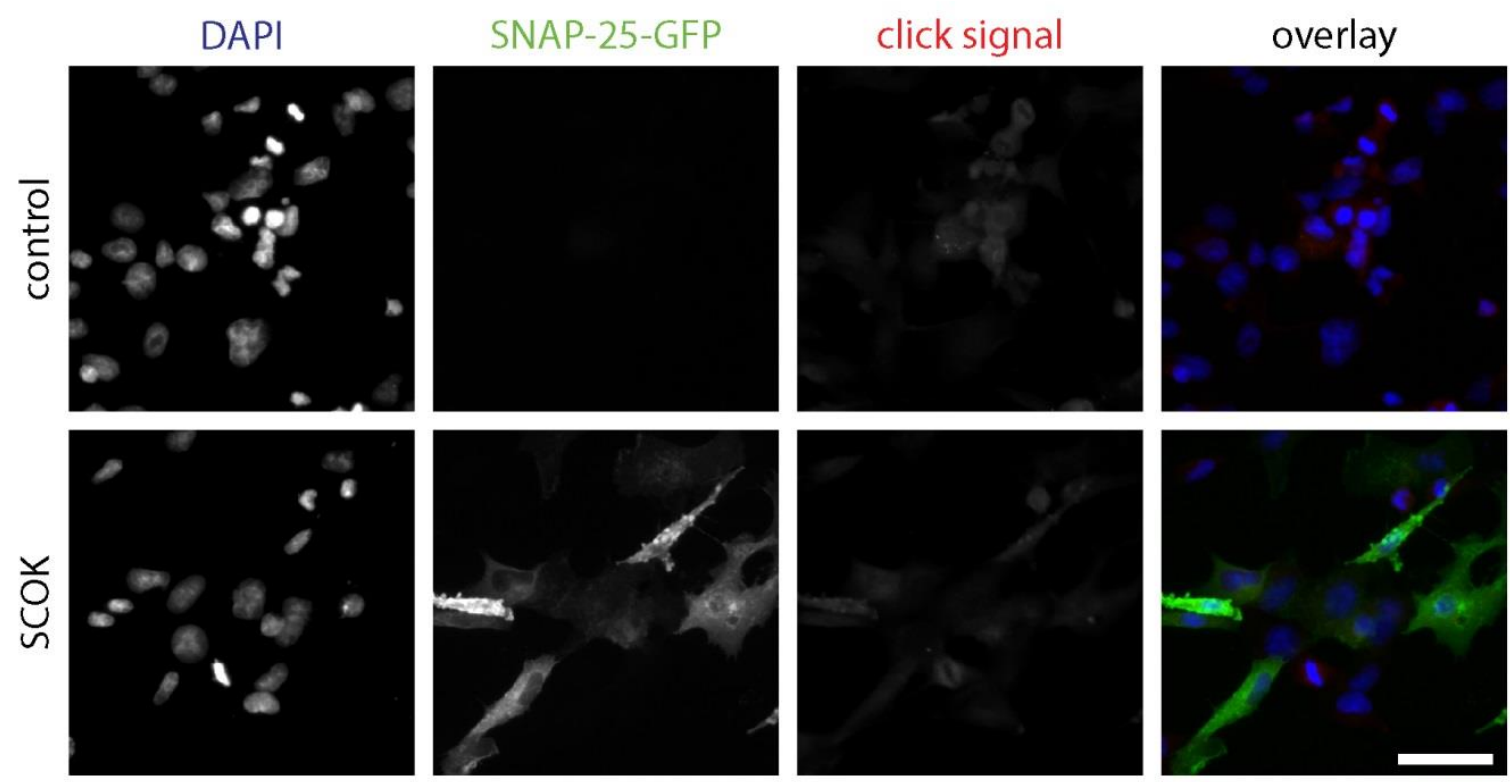

Figure 3-9 SCOK incorporation and click reaction reaction results in nonspecific labeling

BHK cells were transfected with SNAP-25-GFP M219TAG and the mutant version of the PylRS $^{\text {AF}} /$ tRNA pair (Plass et al., 2012) in the presence of SCOK $(250 \mu \mathrm{M})$ or in its absence, as a control. Upon fixation and permeabilization, click reaction was performed for 30 minutes at room temperature with $50 \mu \mathrm{M}$ KK114-azide. DAPI staining was performed to reveal all cell nuclei. All the images taken in a specific fluorescence channel are identically scaled. Scale bar, $40 \mu \mathrm{m}$.

Once I eliminated the SCOK random presence in all cells as a contributing factor to the high background, there are two more possible causes for it. First, I would mention the different blocking conditions: I employed only 1\% FCS in PBS instead of 5\% BSA-5\% peptone used for blocking before CuAAC. Second, the high concentration of KK114-azide $(50 \mu \mathrm{M})$, which is the maximum that I have optimized for the $\mathrm{CuAAC}$, is probably too high for these milder blocking conditions. However, in the absence of a metal ion catalyzer, the SPAAC reaction requires the use of a high concentration of dye in order to push the equilibrium toward the final labeling product. As a result, when I used lower dye concentrations this indeed diminished the background but did not reveal any specific staining (data not shown). 


\section{Results}

I have also tried to incubate the samples at higher temperatures $\left(37^{\circ} \mathrm{C}\right)$ and longer incubations ( 2 hours up to overnight) without satisfactory results.

In conclusion, I excluded the SCOK reaction with azide derivatives of fluorescent dyes as a plausible method for labeling proteins for super-resolution microscopy investigations.

\section{BCNK (bicyclo[6.1.0]nonyne-L-lysine)}

Another amino acid I tested for copper-free click chemistry is BCNK presented in literature as a more reactive variant of SCOK (Lang et al., 2012; Plass et al., 2012). Incorporation of BCNK in BHK cells expressing SNAP-25-GFP M219TAG is relatively efficient as proven by the GFP signal (Figure 3-10 A). For this SPIEDAC reaction the concentration dye required is very low: I used only $100 \mathrm{nM}$ Atto-tetrazine (more than 100-fold lower than of the azide normally used in SPAAC or CuAAC). Also, due to the reactivity of the tetrazine moiety, only 10 minutes at a temperature of $37^{\circ} \mathrm{C}$ should be suffiecient for a complete reaction. However the SPIEDAC click reaction with Atto647N-tetrazine is not efficient, as no specific signal from the transfected cells can be detected. What is more, this labeling reaction presents a very strong background in all the cells incubated with $\mathrm{BCNK}$, but to a lower extent in the controls where the ncAA was absent.

The high background in Figure 3-10 has a perinuclear/cytoplasmic distribution, presumably due to the accumulation of the BCNK in all treated cells. So I decided to also perform the SPIEDAC reaction on membrane sheets, where no cytoplasmic contaminants should be present. I expressed the membrane attached protein SNAP-25-GFP in BHK cells and subjected them to sonication (see Figure 2-2 for the setup used in this procedure). The ultrasound pulse removes most of the cells, leaving behind only the plasma membranes well adhered to the PLL-coated coverslips (Avery et al., 2000). However, even in this case I could not detect any specific labeling of the overexpressed SNAP-25-GFP, even though I increased the dye concentration to $200 \mathrm{nM}$ Atto647N-tetrazine (Figure 3-10 B). The STED image shows that Atto647N-tetrazine is prone to aggregation (see the bright spots in the image). As a positive control, I performed the CuAAC labeling of PRK incorporated into the same protein (i.e. SNAP-25-GFP) using $25 \mu \mathrm{M}$ Star635P-azide for 30 minutes at RT. In this case I obtained efficient labeling with good correlation between the GFP and the click signal. 

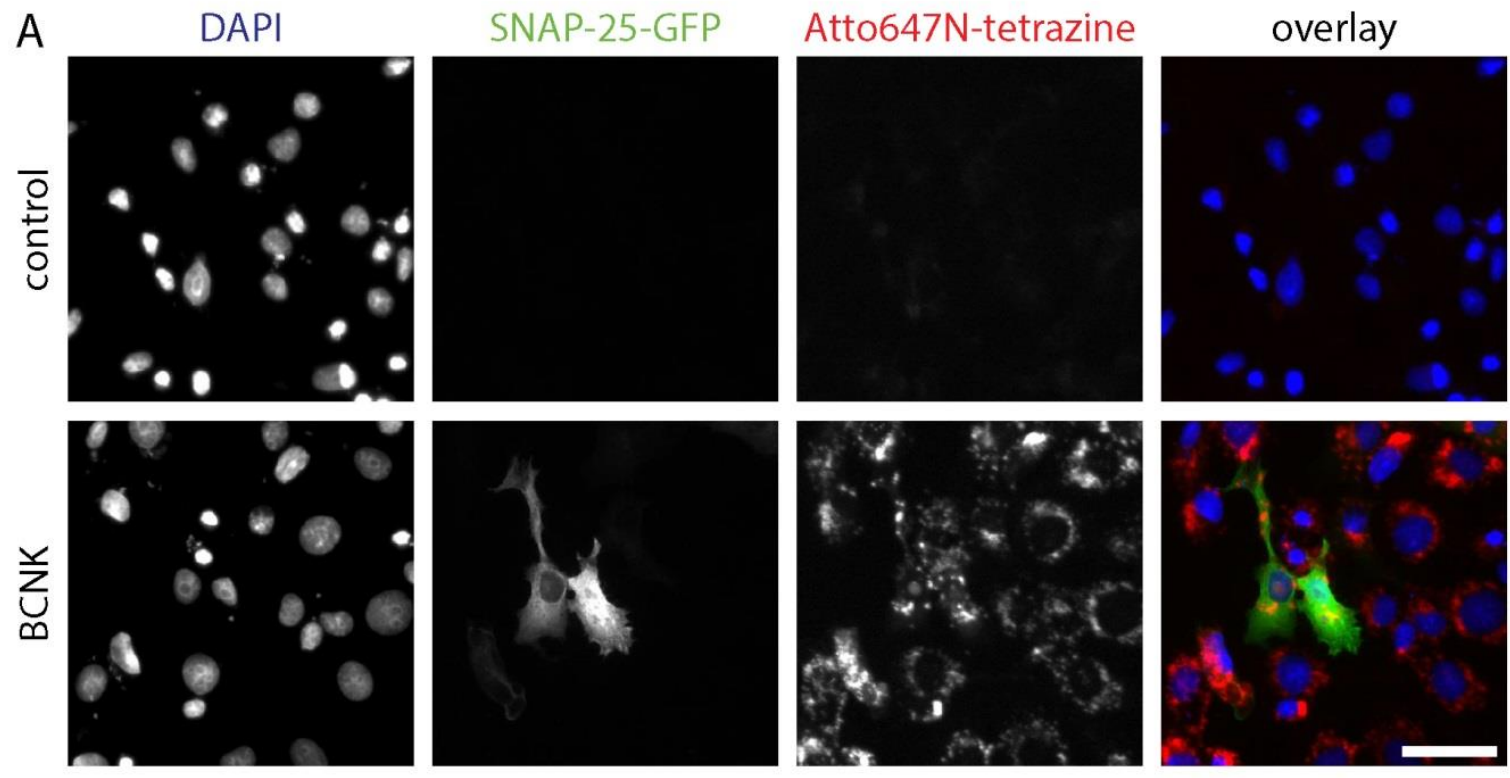

B

Atto647N-tetrazine
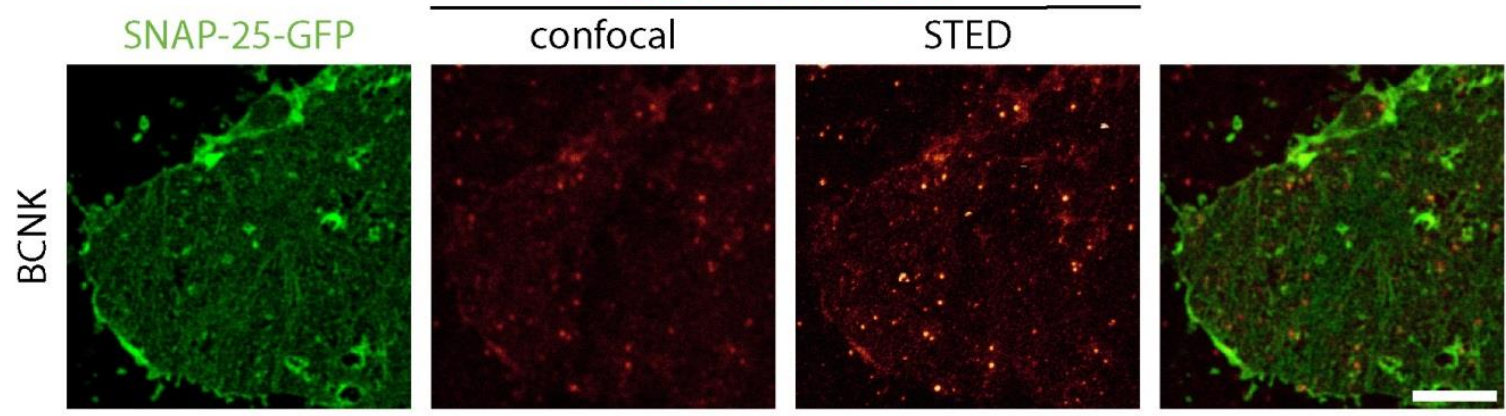

C
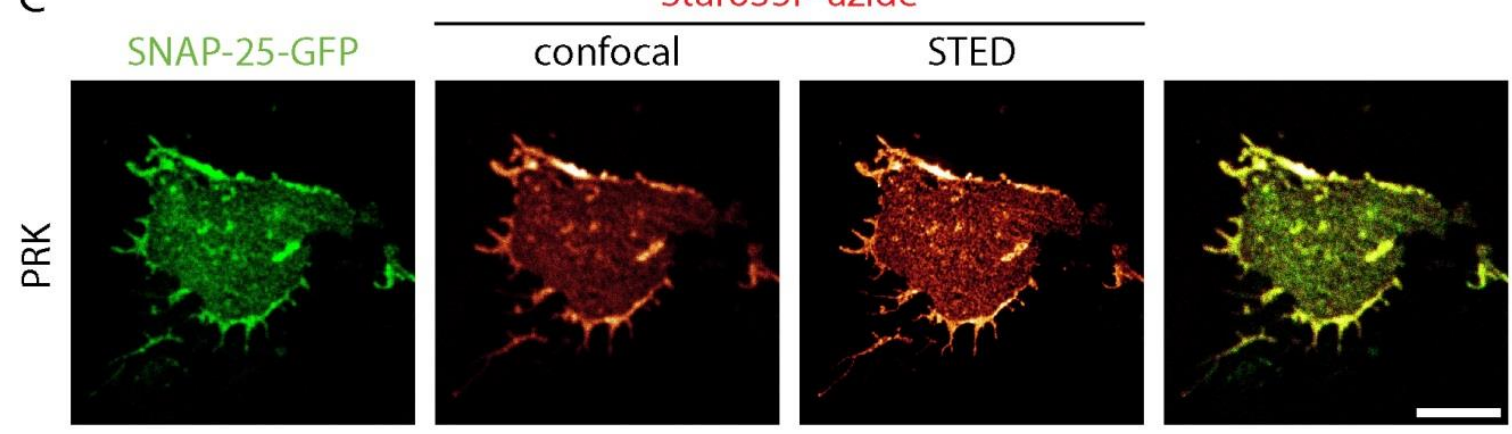

Figure 3-10 BCNK incorporation and click chemistry shows unspecific labeling

BHK cells were allowed to express SNAP-25-GFP M219TAG, in the presence or absence of ncAAs, in order to test the efficiency of the SPIEDAC versus the CuAAC click reaction. The ncAA BCNK and PRK were fed to the cells at a final concentration of $250 \mu \mathrm{M}$ in the medium. A. Representative epifluorescence microscopy images of cell that have incorporated BCNK or not (i.e. controls where no ncAA was added). Both the BCNK-treated samples and the controls were incubated with $100 \mathrm{nM}$ Atto647N-tetrazine (in the absence of any metal-ion catalyzer) for 10 minutes at $37^{\circ} \mathrm{C}$. Cell nuclei are shown using DAPI staining. Scale bar, $40 \mu \mathrm{m}$. B-C. Membrane sheets were generated from cells that had incorporated either BCNK (B) or PRK (C) into SNAP-25-GFP. Upon fixation, the samples were clicked with $200 \mathrm{nM}$ Atto647N-tetrazine for 10 minutes at $37^{\circ} \mathrm{C}(\mathbf{B})$ or with $25 \mu \mathrm{M}$ Star635P-azide for 30 minutes at RT (C) under copper-catalyzed conditions. Membrane sheets were imaged in the 


\section{Results}

confocal mode for GFP and Atto647N or Star635P channels, as well as in the STED mode for the red fluorophores. The overlays consist of the superposition of the confocal GFP images with the STED ones. Scale bars for B-C, $5 \mu \mathrm{m}$.

Therefore, even though $\mathrm{BCNK}$ is efficiently incorporated into the protein of interest tested here, the SPIEDAC reaction it undergoes with Atto647N-tetrazine is unsatisfactory. So, below I tried to use the more hydrophilic KK114-tetrazine, in the hope that this might reduce the nonspecific background and bring forward the specific signal from SPIEDAC.

\section{TCOK (trans-cyclooct-2-ene-L-lysine)}

The most recent ncAA reported to be suitable for copper-free click chemistry is TCOK (trans-cyclooct-2-ene-L-lysine; refer to Figure 3-5 B to see its chemical structure). To easily compare the incorporation efficiency and SPIEDAC labeling reaction of the three strained ncAAs, I transfected cells with $\alpha$-synuclein-GFP G141TAG together with the $\mathrm{PylRS}^{\mathrm{AF}} / \mathrm{tRNA}$ pair to drive the incorporation of BCNK, SCOK, and TCOK (see Figure 3-11 B-D). The control condition, in which the cells were transfected with the same constructs but no ncAAs was provided during protein expression, is shown in Figure 3-11 A. I fixed and permeabilized all samples before performing click reaction with $1 \mu \mathrm{M}$ KK114tetrazine. Notice that I used a 10-fold higher concentration for the dye than in Figure 3-10, in the attempt to obtain quantitative labeling. In addition, this concentration was used for live labeling of BCNK and TCOK (Uttamapinant et al., 2015).

All three ncAAs are efficiently incorporated into the target proteins, but only SCOK and TCOK show detectable signal in SPIEDAC and only to a partial extent (Figure 3-11). For instance, inspite of the high KK114-tetrazine concentration, very little signal can be detected - notice how only the cell with the highest $\alpha$-synuclein-GFP levels can be seen in the click channel (middle panels in Figure 3-11 C). In contrast, the KK114 fluorescence is detectable not only in the cells that express the protein of interest, but also in the surrounding nontransfected cells (Figure 3-11 D). As in the control (Figure 3-11 A) there is no background staining, I can infer that the nonspecific labeling in Figure 3-11 D arises from free TCOK. This ncAA is still trapped in the cells and was not effectively washed out during the 2 hours incubation in normal medium, prior to fixation. Indeed, this observation has also been reported by Uttamapinant et al., 2015. 

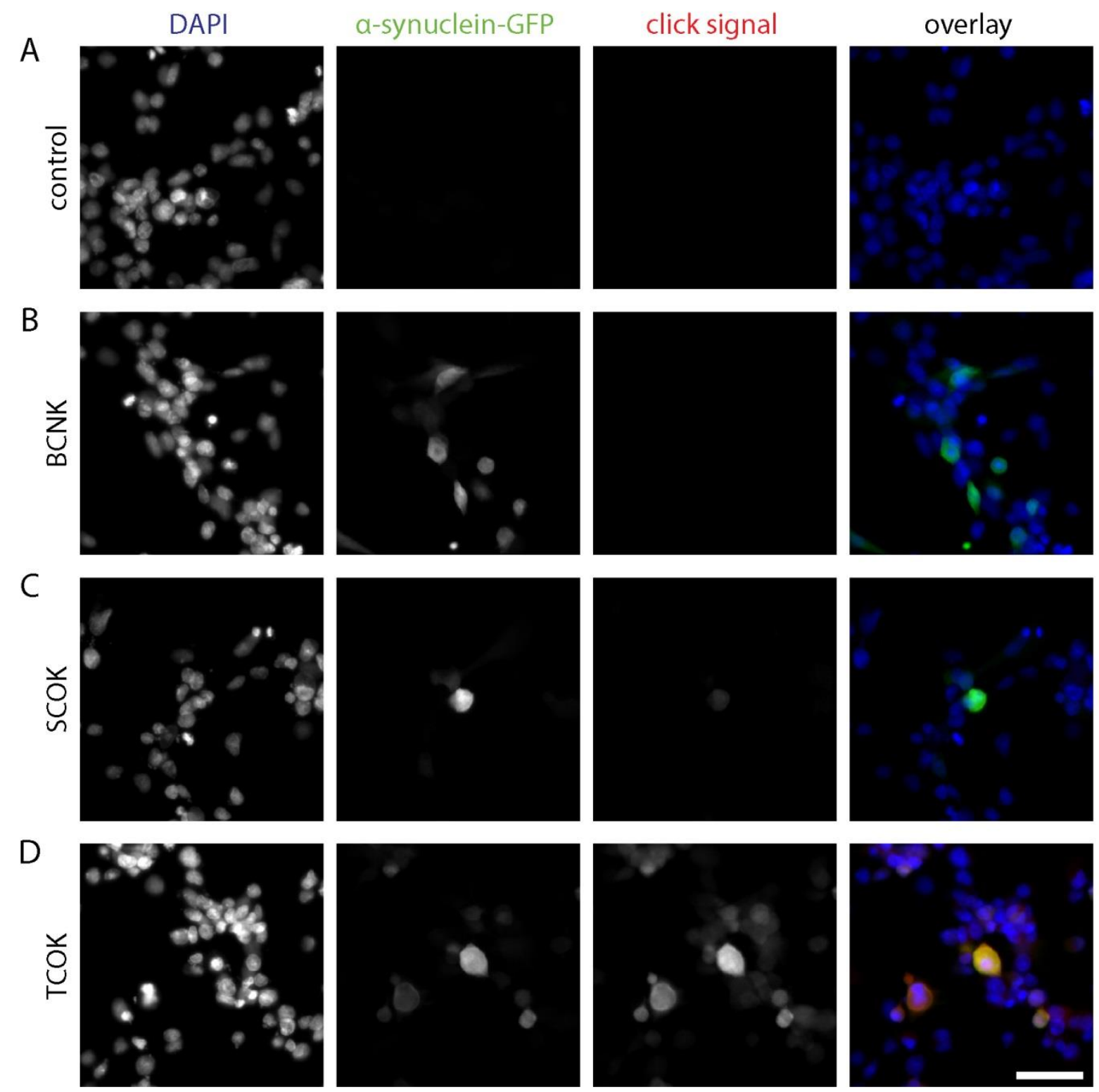

Figure 3-11 No specific labeling in copper-free click reaction for BCNK, SCOK, and TCOK

All three ncAAs copper-free click are efficiently incorporated into the target proteins but only TCOK undergoes specific click reaction (to some extent). Representative images of BHK cells that have expressed for 18 hours $\alpha$-synuclein-GFP G141TAG and PylRS ${ }^{\mathrm{AF}} / \mathrm{tRNA}$ pair in the absence of any ncAA (A) or in medium supplemented with $1 \mathrm{mM} \mathrm{BCNK}(\mathbf{B})$, SCOK $(\mathbf{C})$, or TCOK (D). The ncAA excess was washed off during a 2-hour incubation with normal medium. Subsequently the cells were fixed, permeabilized and subjected to copper-free click reaction with $1 \mu \mathrm{M}$ KK114-tetrazine for 30 minutes at $37^{\circ} \mathrm{C}$. Nuclei were stained with DAPI. The images were scaled identically for the DAPI, GFP, and KK114 channel, respectively. The scale bar represents $40 \mu \mathrm{m}$ and applies to all images.

As a measure to improve this labeling reaction, I also tried to lower the tetrazine-dye concentrations to $100 \mathrm{nM}$ or $200 \mathrm{nM}$. The background decreased to some extent but so did the labeling and it was clear that the alkyne epitopes were not quantitatively labeled (data not shown). 


\section{Results}

To conclude, I did not obtain satisfactory results in the optimization of the copper-free labeling in none of the amino acids reported to undergo copper-free click chemistry reactions. Refer to Table 3-1 for a summary of the results for the ncAA testing and optimization. Therefore, as their labeling reactions were by far neither quantitative nor specific enough, I decided not to use them further in this project.

Table 3-1 Summary for the click reaction specificity for the tested ncAAs

\section{ncAA Copper-catalysis Click reaction type Labeling result}

\begin{tabular}{|llll|}
\hline pAzpa & + & CuAAC & Poor signal-to-noise ratio \\
\hline AZK & + & CuAAC & No specific signal detected \\
\hline BCNK & - & SPAAC & No specific signal detected \\
\hline \multirow{2}{*}{ PRK } & + & SPIEDAC & No specific signal detected \\
\hline SCOK & - & CuAAC & $\begin{array}{l}\text { Specific signal with excellent } \\
\text { signal-to-noise ratio }\end{array}$ \\
\hline TCOK & - & SPAAC & No specific signal detected \\
\cline { 2 - 4 } & & SPIEDAC & Low labeling levels \\
\hline
\end{tabular}

\subsubsection{Optimizing the Labeling of PRK}

As PRK was the non-canonical amino acid that provided the most reliable labeling in click reactions among all the tested compounds, I decided to pursue this research line and optimize its reaction with fluorescent dyes.

For PRK, the best incorporation in the proteins occurs at a final concentration of $250 \mu \mathrm{M}$ in the cell medium (data not shown). In addition, it is beneficial to use media in the absence of antibiotics as this reduces cell death caused by the transfection procedure. The next step was to optimize the click reaction by adjusting the dye hydrophilicity and concentration during the click reaction.

To identify better dye candidates, I compared the labeling efficiencies of Atto647N-azide, KK114-azide, and Star635P-azide in CuAAC. While KK114 and Star635P are very soluble in water and in PBS, Atto647N is rather hydrophobic and requires organic solvents for solubilization (Wurm et al., 2012). As a result, the highest amount of Atto647 derivatives (e.g. azide or alkyne) I could use with a fairly good signal-to-noise ratio was $2 \mu \mathrm{M}$. When 
applied in higher concentrations, the Atto647N strongly binds to membranes thereby causing unspecific labeling (Hughes et al., 2014).

In order to make sure that all the proteins that had incorporated PRK were fully labeled, I resorted to the new generation coumarin dyes KK114 and Star635P which have comparable brightness and stability, while at the same time, being more biocompatible due to their excellent solubility under physiological conditions and low unspecific interactions with biological material (Kolmakov et al., 2010).

In Figure 3-12, a direct comparison between Atto647N and KK114 is made for the coppercatalyzed labeling of PRK incorporated in SNAP-25-GFP. Even though the concentration of KK114-azide used for the click reaction is more than twice that of the Atto647N-azide, the background for the click signal is minimal (compare the click signal panels in Figure 3-12 A and B). The Atto647N dye binds considerably even to cells which were not incubated with the ncAA (control; upper panels in Figure 3-12 A), attesting for its unspecific binding to cellular material. In addition, for the PRK treated samples there is considerable Atto signal in cells that do not express GFP (i.e. non-transfected). In contrast, the click signal provided by KK114-azide is highly specific with almost no background in the control or in the nontransfected cells (see Figure 3-12 B).

In Figure 3-12, note that the higher signal in the GFP signal in the control could indicate a bleed-through of Atto fluorescence into this channel. 


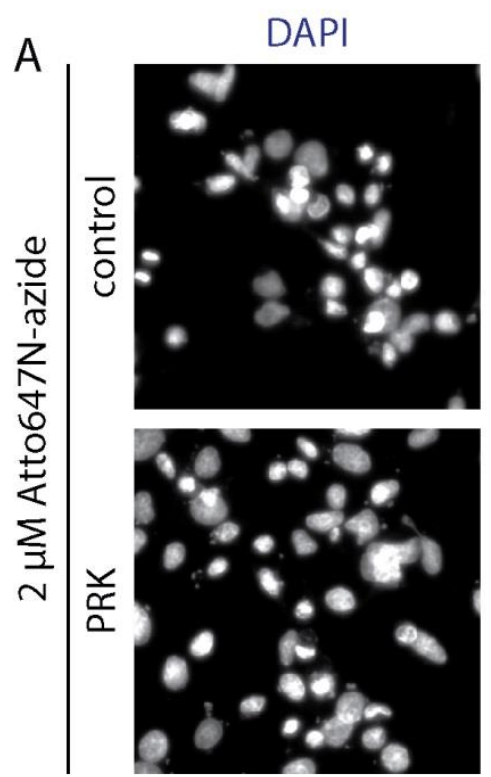

SNAP-25-GFP
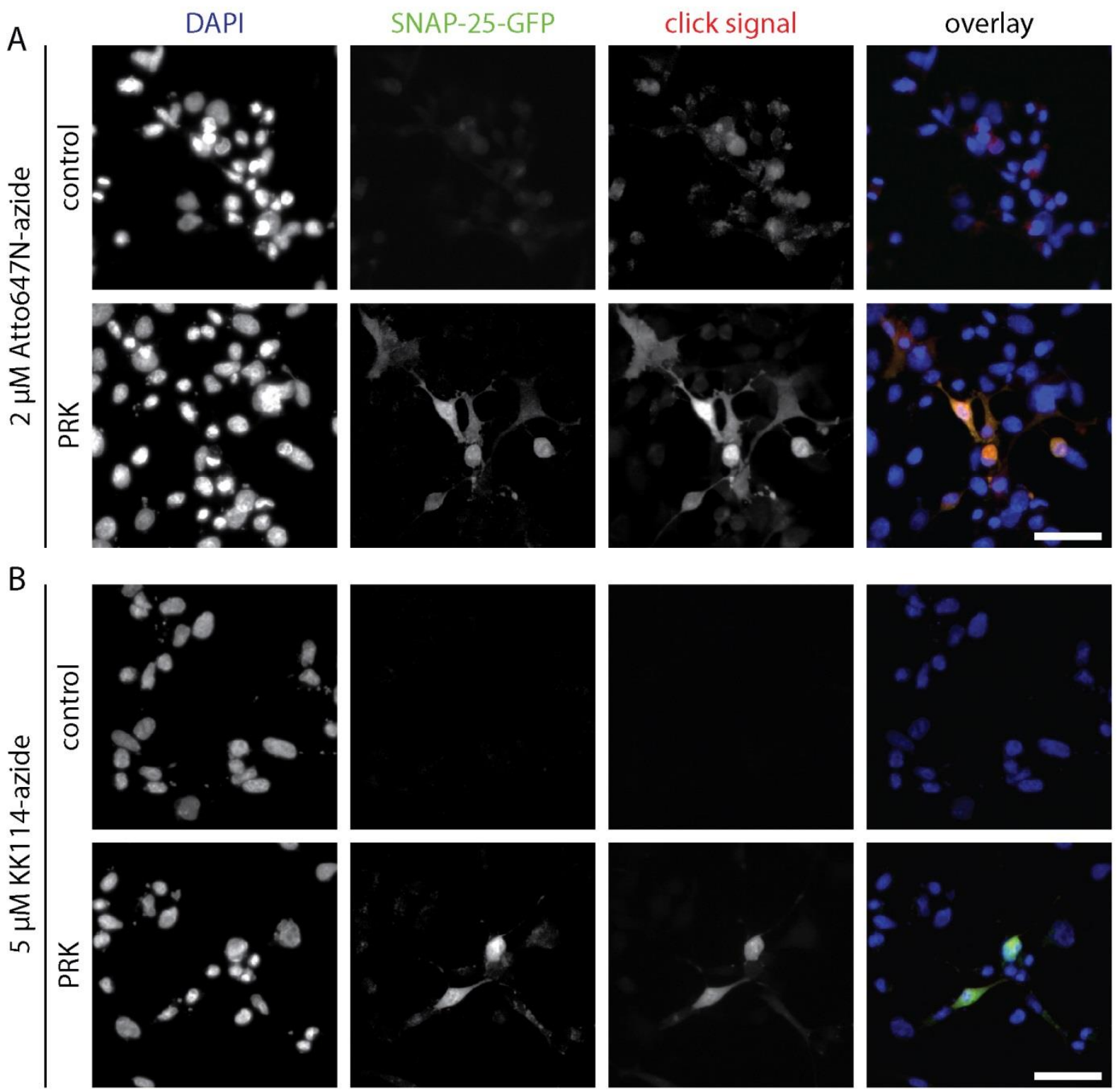

Figure 3-12 Labeling specificity Atto647N-azide and KK114-azide

PRK was incorporated into SNAP-25 M219TAG by BHK cells. The samples were fixed, permeabilized, and subsequently labeled using CuAAC with $2 \mu \mathrm{M}$ Atto647N-azide (A) or $5 \mu \mathrm{M}$ KK114-azide (B). Control samples represent cells that were transfected in the absence of any ncAA (upper panels), while PRK was added in the medium the cells were transfected for the lower panels. All corresponding channel were identically scaled for comparison purposes. Scale bars, $40 \mu \mathrm{m}$.

In view of the above, I ruled out Atto647N as a click stain and decided to further improve the click reaction using hydrophilic dyes. Figure 3-12 clearly shows that for these identically scaled images, the intensity of the Atto staining is higher than the KK114 one (compare the click signal images in Figure 3-12 A versus B). However, the two fluorophores themselves have comparable brightness (Kolmakov et al., 2010). Therefore, only insufficient labeling could be responsible for this observation. 
As a next step, I therefore optimized the dye concentration for the click reaction. I used the KK114 dye only for initial experiments and then I switched to the commercially available Star635P-azide. The latter synthetic fluorophore is designed to have an even lower fluorescent background and offer a better contrast in cellular stainings (Kolmakov et al., 2012). Figure 3-13 illustrates the labeling parameters for an optimal click staining: 30 minutes incubation time with $50 \mu \mathrm{M}$ Star635P-azide. The click signal reaches saturation already at $25 \mu \mathrm{M}$ (data not shown), indicating that the reaction is complete. Also, by analyzing cell expressing GFP-tagged $\alpha$-synuclein and at the same time labeled in a coppercatalyzed click reaction with Star635P, it is evident that this reaction offers a very accurate and proportional labeling compared with the GFP signal (see Figure 3-13 A). The very good correlation between the GFP (i.e., the ncAA-containing protein) and the click signal can be visually assessed in Figure 3-12 B. The transfected cell shows comparable GFP and click signal, whereas the surrounding non-transfected cells are not labeled, as should be the case.

A
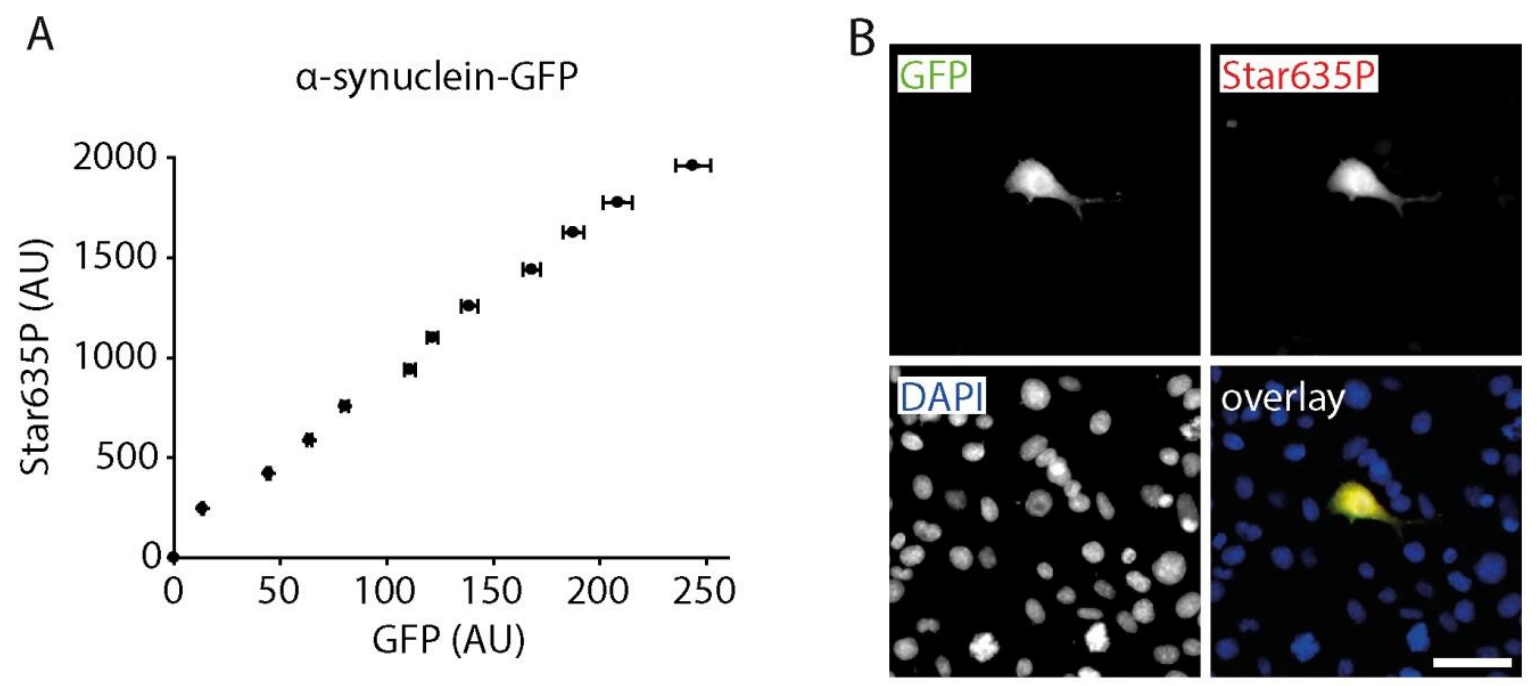

Figure 3-13 Optimal labeling parameters using PRK and Star635P-azide

The click labeling reaction was optimized and the best conditions are shown. BHK cells expressing $\alpha$ synuclein-GFP G141TAG and the bioorthogonal PylRS/tRNA for PRK incorporation were fixed and permeabilized then clicked with $50 \mu \mathrm{M}$ Star635P-azide for 30 minutes at room temperature. A. The graph shows the intensity of the Star635P-azide fluorescence plotted against the GFP fluorescence intensity. There is clear linear correlation of the Star635P fluorescence with the fluorescence of the $\alpha$ synuclein-GFP. This corroborates with the fact that the reaction is not only specific, but also very efficient. The symbols depict fluorescence intensity averages derived from 172-1902 cells from a single typical experiment. B. The images show cells that have been allowed to incorporate PRK into $\alpha-$ synuclein-GFP and were later click labelled with Star635P-azide and DAPI stained. The GFP expressing cell shows strong labeling with Star635P. Scale bar, $40 \mu \mathrm{m}$. 


\subsection{ClickOx as a Possible Tool to Reduce Oxidative Stress}

For possible in vivo applications of the copper-catalyzed click reaction as well as for a better structural integrity of the samples, it is useful to reduce the unwanted damage caused by the free radicals, especially reactive oxygen species (ROS). These are produced during the click reaction by the reduction of $\mathrm{Cu}^{2+}$ to $\mathrm{Cu}^{+}$. Recently, an alternative strategy to counter these ROS has been proposed, termed "ClickOx" (Löschberger et al., 2014). It involves the use of a radical scavenging system composed of $\beta$-D-glucose, glucose oxidase and catalase, first developed for polarography applications (Benesch and Benesch, 1953).

I tested this system under the conditions I normally employ for the copper-catalyzed click reaction $(\mathrm{CuAAC})$. By analyzing images taken in the presence or in the absence of the ClickOx system, I could find no difference in the intensity profiles obtained or in the morphology of the cells (Figure 3-14 A). Comparing the graphs from Figure 3-14 C and D, it becomes apparent that the presence of the oxygen scavenger system does not improve the labeling intensities for the synthetic dye, nor does is it protect the GFP fluorophore to any measurable extent. According to the protocol employed by Sauer and coworkers, the click reaction was allowed to proceed for only 10 minutes (Löschberger et al., 2014). However, when I optimized the click reaction (see Section 3.2.3), I established that incubations shorter than 30 minutes are not sufficient for appropriate labeling. Moreover, if I use concentrations lower than $5 \mathrm{mM}$ of $\mathrm{Cu}^{2+}$ (I tested for instance the $1 \mathrm{mM}$ used by Sauer and coworkers), then the efficiency of the click reaction plummets (data not shown).

In conclusion, the ClickOx system does not bring any improvement under the conditions that I have optimized for proper click labeling, namely 30 minutes incubation time and $5 \mathrm{mM}$ concentration of $\mathrm{Cu}^{2+}$. So I did not use it in further experiments. 

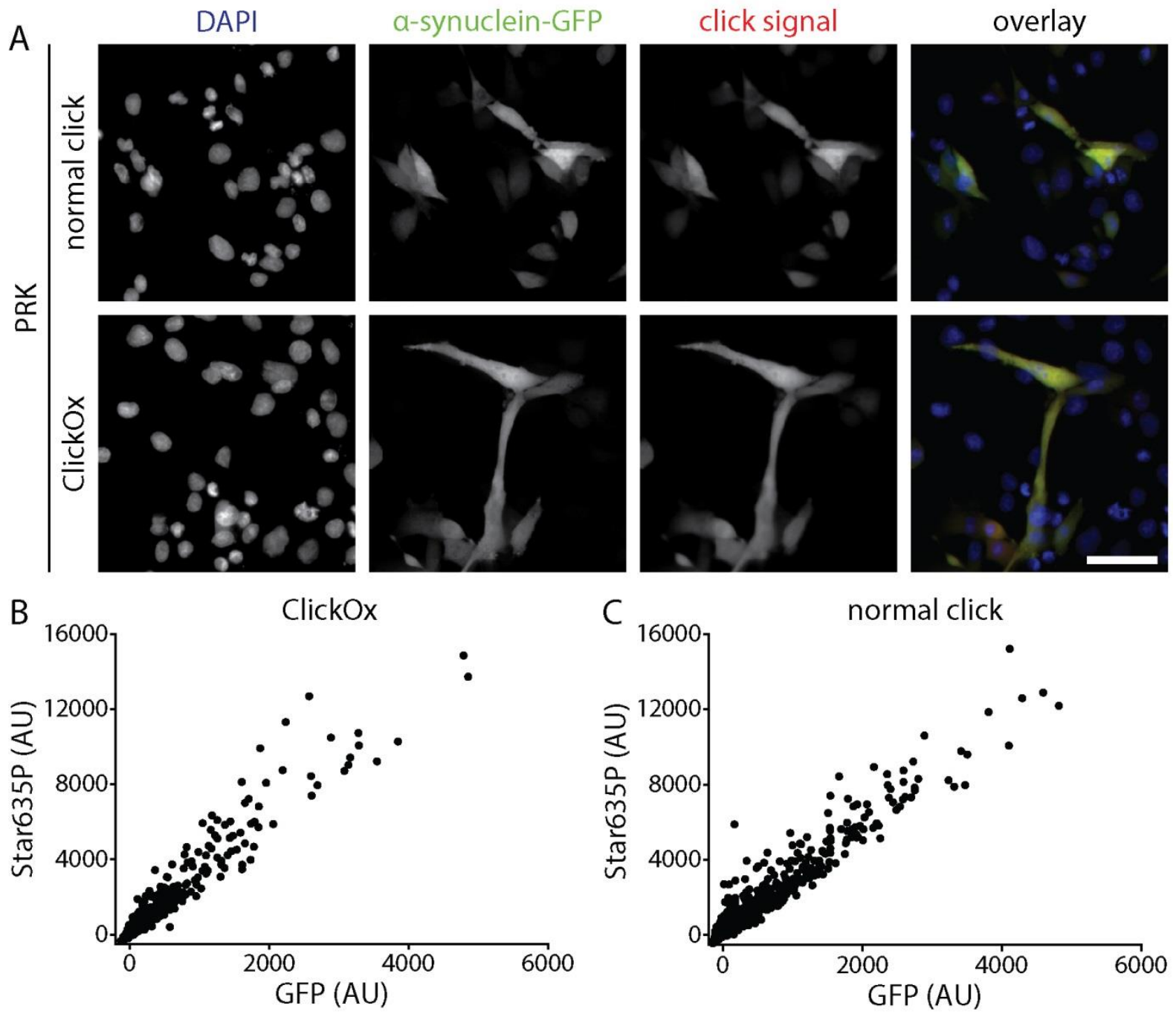

Figure 3-14 Similar labeling intensities for ClickOx and normal samples

Cells that have incorporated PRK into $\alpha$-synuclein-GFP G141TAG were permeablized post-fixation and subjected to click labeling for 30 minutes with $25 \mu \mathrm{M}$ Star635P-azide in the presence of an oxygen scavenger system (ClickOx) or without it (normal click). A concentration of $5 \mathrm{mM} \mathrm{Cu}^{2+}$ was used in the click mix for both normal and ClickOx conditions A. Representative images in the DAPI, GFP and Star635 channels, as well as their overlays are shown. The images in each of the channels were scaled identically. Scale bar, $40 \mu \mathrm{m}$. B-C. Graphs showing Star635P intensity as a function of GFP fluorescence intensity. Note that no difference in labeling intensity and cell morphology can be observed between samples treated with ClickOx versus normal samples. 


\subsection{Incorporation of PRK into the Proteins of Interest}

After establishing that PRK is the best candidate amino acid for the goals of this project and optimizing its reaction with azide derivatives of fluorescent dye in $\mathrm{CuAAC}$, I wanted to ascertain that it is also properly incorporated into the 26 proteins of interest. So I tested the constructs I have previously generated or obtained from other researchers (see Section 3.1). For this, I have used the FP-tagged versions of the proteins that would report on the incorporation of PRK using the fluorescent protein fluorescence intensity as a readout. As mentioned above, because the FP tag is located C-terminally with respect to the Amber stop codon, it acts as a reporter for ncAA incorporation. Therefore, the more intense the FP signal in the transfected cells, the better the incorporation.

As can be seen in Figure 3-15, PRK is properly incorporated in all the 26 proteins tested here. The control samples were transfected under the same conditions, but the ncAA PRK was not provided in the cell medium. These controls show no FP signal, confirming that Amber stop codon suppression occurs only in cells that have not only the enzymatic machinery (i.e., the PylRS/tRNA pair) but also the bioorthogonal ncAA. Therefore, the incorporation of PRK is specific.

Another parameter to be taken into account is the size of the protein, which inversely correlates with the expression level. Namely, the smaller the protein, the higher the expression level. However, the latter statement holds true only if the same promoter is used to drive the expression of these proteins. In this project, all proteins are cloned in vectors where the human cytomegalovirus (CMV) promoter drives transcription. This enables a reliable comparison between the expression levels of the different proteins. 

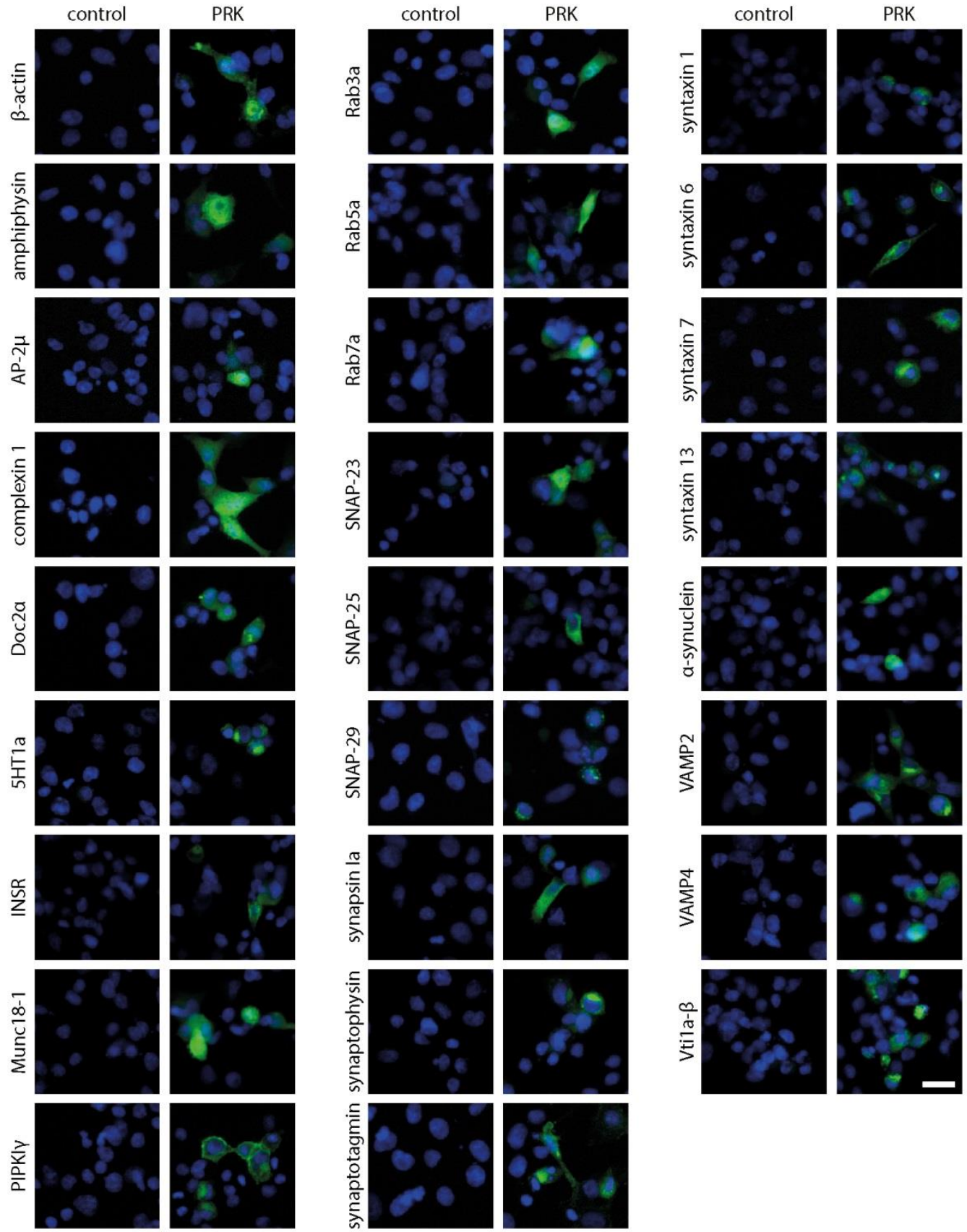

Figure 3-15 PRK is well incorporated in all 26 proteins included in this study

The specificity of PRK incorporation was assessed by cotransfecting cells with the PylRS/tRNA plasmid as well as different plasmids encoding for the FP tagged proteins of interest. The samples that were transfected but grown in the absence of ncAAs represent controls where no FP fluorescence should be detected. Left-hand and right-hand panels show representative images of cells deprived of PRK or exposed to it, respectively. Scale bar, $20 \mu \mathrm{m}$. 


\subsection{Investigating Protein Cluster Organization}

In the next chapters, I will elaborate how I employed the previously optimized labeling paradigm (see Figure 3-16). I used it to investigate in super-resolution microscopy whether the presence of the fluorescent protein (FP) tags affects the organization of the protein assemblies formed by each of the 26 proteins included in this study. This technical approach relies on the genetic encoding (or incorporation) of PRK into the proteins of interest followed by copper-catalyzed click reaction with azide derivatives of fluorescent dyes after the fixation and permeabilization of the samples. The main question behind this experiment is whether the protein assemblies formed by FP chimeras differ in size and composition compared with their non-tagged counterparts.

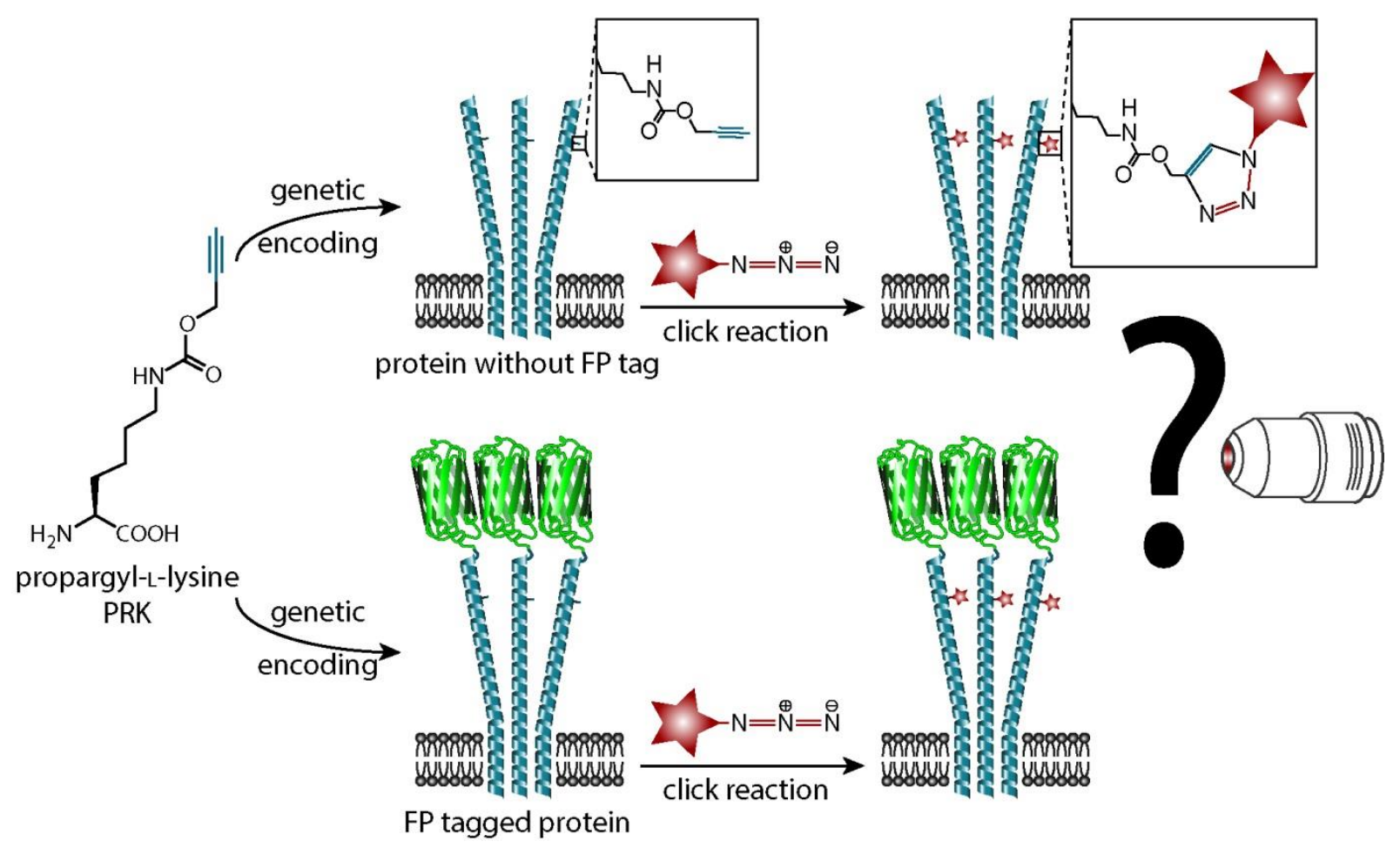

Figure 3-16 Genetic encoding of PRK and click reaction with azide derivatives for labeling protein assemblies for nanoscopy investigations.

Propargyl-L-lysine (PRK) is incorporated in the proteins of interest by adding it to the culture medium of cells expressing the proteins of interest either without a fluorescent protein (FP) tag (upper panel) or with an FP tag (lower panel). The resulting alkyne derivatized proteins are subsequently fixed and labeled in a copper-catalyzed click reaction. Appropriate dyes for super-resolution imaging are employed: Star635P-azide for STED microscopy and Alexa647N-azide for GSDIM. 
To test whether this system is suitable for super-resolution imaging, I checked if the mutants that performed best in epifluorescence microscopy delivered good labeling results under confocal and STED imaging conditions. As can be observed in Figure 3-17 A, there is a correlation between the GFP signal and the one of the Star635P dye. This indicates that the click reaction works efficiently and the concentration is optimal (saturates all alkyne groups presented by the protein of interest). After imaging the samples at a higher magnification, the differences in the resolution of the images in confocal and STED become apparent (compare the two panels in Figure 3-17 B). The ability to discern finer details and structures in the STED image compared to the confocal one is even more evident when a smaller area is enlarged (Figure 3-17 C).

In this STED experiment, the resolution achieved is approximately $\sim 60 \mathrm{~nm}$. The resolution in the xy plane (or lateral plane) is also affected by the considerable lower resolution in the $\mathrm{z}$ axis (cca $700 \mathrm{~nm}$ in the $\mathrm{z}$ compared with $70-100 \mathrm{~nm}$ in the xy plane). This makes it difficult to clearly distinguish the structures in the center of the cell from Figure 3-17 B. But in the periphery of the same cell the contrast is much better due to the smaller thickness of the cell in these regions. This issue can be avoided if the samples are embeded in a plastic resin (e.g. melamine) and cut into thin sections at the ultramicrotome (Revelo and Rizzoli, 2015).

The highest attainable resolution is required in order to distinguish between the small differences in the macromolecular assemblies of the proteins of interest. Therefore, in the final measurements I used STED and GSDIM setups with lateral resolutions of approximately $30 \mathrm{~nm}$ and $20 \mathrm{~nm}$, respectively (see Sections 3.5.1 and 3.5.2). I analyzed the spots detected using the above-mentioned microscopy techniques (for details refer to Section 2.14.2). The main parameters taken into consideration were the full width at half-maximum (FWHM), which is a good approximate of spot size, the peak intensity, and the total spot intensity. Refer to Figure 2-3 for a graphical summary how the parameters used for data analysis were obtained. 


\section{Results}

A

a-synuclein-GFP

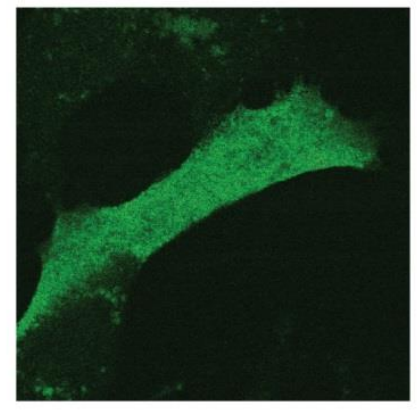

B

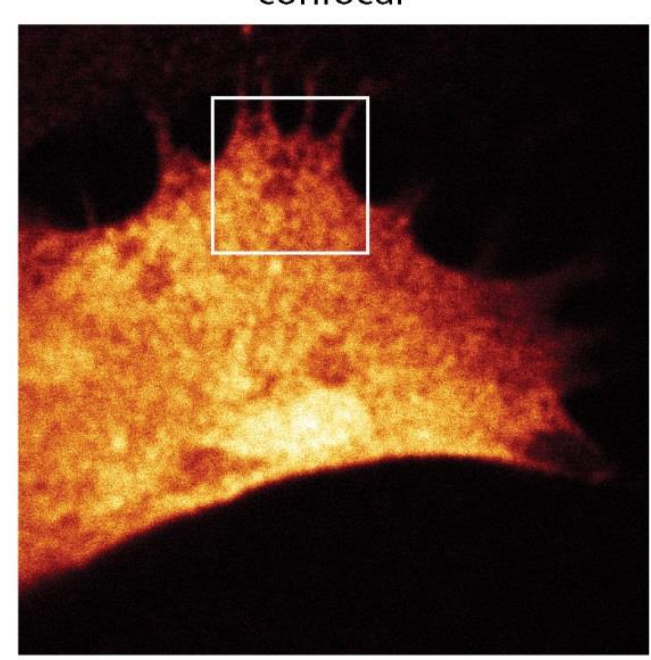

C

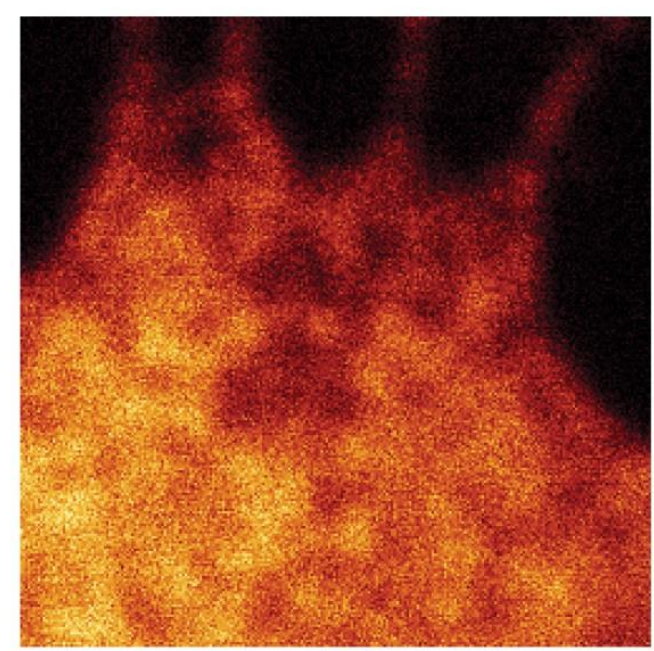

confocal

inset confocal
Star635P-azide

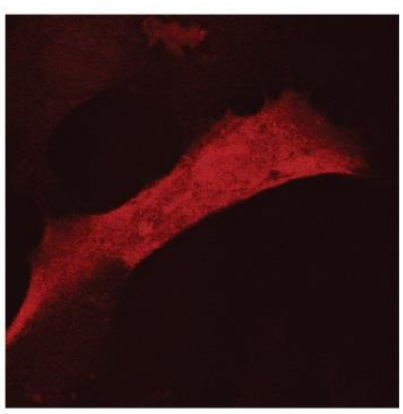

Figure 3-17 Comparison of samples imaged in confocal versus STED

BHK cells were allowed to express $\alpha$-synuclein-GFP G141TAG in the presence of PRK for approximately 18 hours. They were then fixed, permeabilized and labeled with $25 \mu \mathrm{M}$ Star635P-azide. The samples were imaged using a commercial Leica STED microscope at a lower zoom in the GFP and Star635P channels (A), or (B-C) at a higher zoom using either the confocal (left-hand panels) or the STED (right hand panels) mode. The insets in $(\mathbf{C})$ are shown in the images in (B) using white squares. Scale bars for $\mathbf{A}$ to $\mathbf{C}$ are: $10 \mu \mathrm{m}, 5 \mu \mathrm{m}$, and $1 \mu \mathrm{m}$, respectively. 


\subsubsection{Using STED Microscopy}

Using the genetic encoding technique, I incorporated PRK into three neuronal proteins: SNARE proteins SNAP-25 and syntaxin 1, as well as the cytosolic protein $\alpha$-synuclein. For the membrane attached SNARE proteins I used membrane sheets (see Section 2.6) to visualize the assemblies (or clusters) they form in the presence or in the absence of an FP tag. In the case of $\alpha$-synuclein, I used $100 \mathrm{~nm}$ thin sections of cells embedded in melamine (see discussion above).

Representative images of the clusters these proteins form with or without an FP tag can be assessed in Figure 3-18 A. Based on a visual inspection, the differences between the clusters formed by the proteins of interest under these two conditions are minor. This is also confirmed by the analysis of the spot size and peak intensity (Figure 3-18 B-C).

A

$\frac{\text { B means }}{\text { spot size peak }}$

\section{no FP}
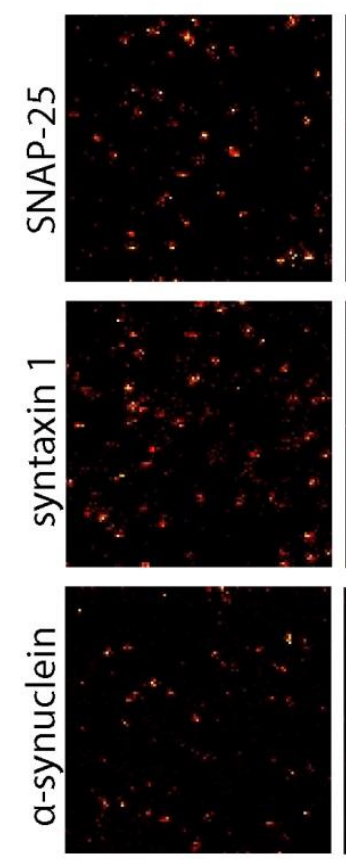

FP tagged
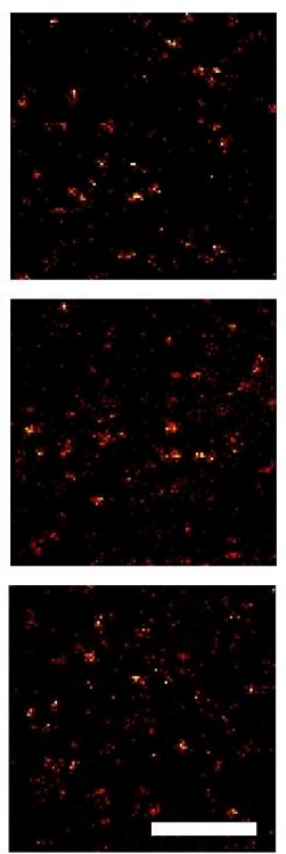

$(\mathrm{nm})$
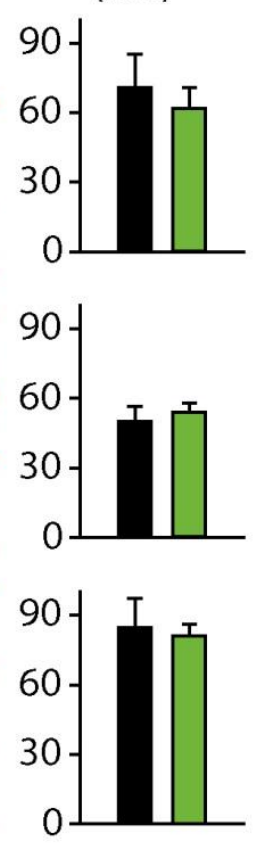

$(\mathrm{AU})$
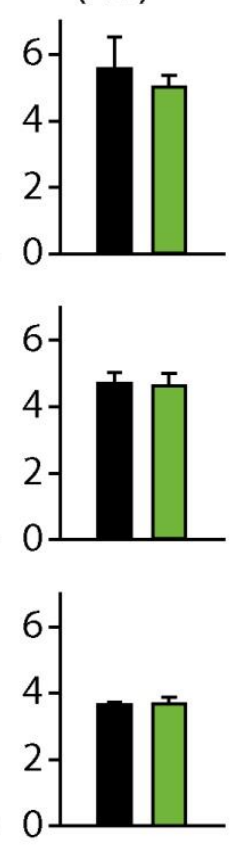

C

medians

spot size peak intensity $(\mathrm{nm})$

$(\mathrm{AU})$
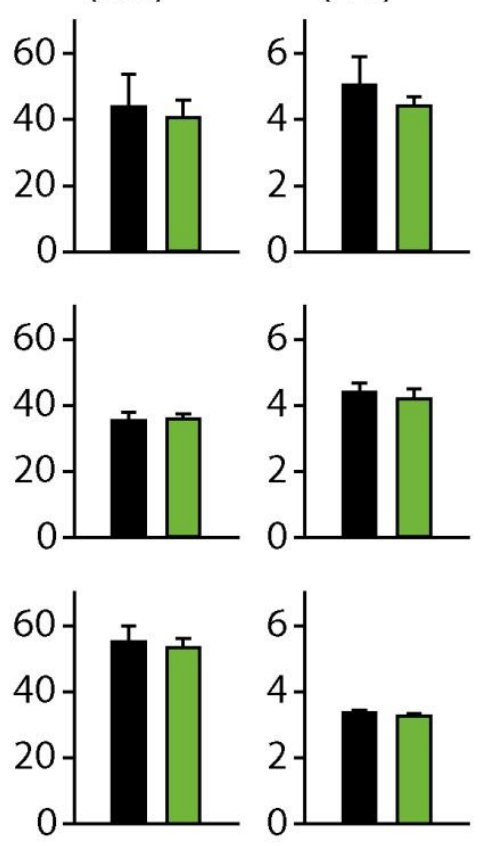

Figure 3-18 The organization of SNAP-25, syntaxin 1, and $\alpha$-synuclein in STED microscopy

A. Images of membrane sheets (for SNAP-25 and syntaxin 1) or $100 \mathrm{~nm}$ thin sections of cells embedded in melamine (for $\alpha$-synuclein) containing the proteins of interest without FP tags (no FP) or as chimeras (FP tagged). All samples were subjected to protein incorporation and click reaction with $50 \mu \mathrm{M}$ Star635P-azide. The Star535P fluorescence is detected using a STED nanoscopy setup that reaches $30 \mathrm{~nm}$ resolution (built by Fabian Göttfert). Scale bar, $500 \mathrm{~nm}$. B-C. Bar plots $( \pm$ SEM) depicting the average values (B) or median values (C) of the spot size (FWHM) or peak intensity. The black bars stand for untagged proteins, while the green bars represent the values for FP tagged proteins. 


\section{Results}

Averages of three independent experiments are shown for SNAP-25 (F84TAG mutant) and syntaxin 1 (V255TAG mutant), while for $\alpha$-synuclein data from four experiments were analyzed (two for each of the G141TAG and T142TAG mutants). None of the differences are statistically significant when subjected to Student's t-test $(\mathrm{P}>0.05)$.

Interestingly, the values for the mean and median in the case of peak intensities are very similar, while the ones for the spot sizes differ considerably. This indicates that there are outliers in the spot size data and this affects the reliability of using the arithmetic mean (or average) as an indicator of where the central tendency for the data lies. In such a case, the median is a more robust approximation for where the middle of the data set is. Therefore, based on the median values, the sizes for the multimolecular assemblies are approximately $40 \mathrm{~nm}$ for SNAP-25 and syntaxin 1, and around $55 \mathrm{~nm}$ for $\alpha$-synuclein. If the mean values are considered, then the average spot sizes would be $70 \mathrm{~nm}$ for SNAP-25, $50 \mathrm{~nm}$ for syntaxin 1 , and $85 \mathrm{~nm}$ for $\alpha$-synuclein.

\subsubsection{Using GSDIM}

I used a similar click based approach to label proteins for GSDIM investigations. The fluorophore of choice in this case was Alexa647, which is widely used in pointillistic superresolution methods. This dye is however sensitive to photodegradation in the presence of oxygen. So, I imaged the samples in a buffer containing ROS scavengers as well as that allow fluorophore recovery (reducing and oxiding reagents; Dempsey et al., 2011).

Initially, I wanted to take advantage of the TIRF imaging mode (with a very good zresolution) and use whole cells for the experiments. However, this resulted in unsatisfactory results (data not shown). Hence I resorted to the same sample preparation as for STED imaging in the case of $\alpha$-synuclein, namely embedding the cells in melamine and thin sectioning them.

I genetically encoded PRK into all the 26 construct pairs (with or without an FP tag) in BHK cells and then I labeled the fixed samples with Alexa647N-azide. The optimal concentration of Alexa647N was $2 \mu \mathrm{M}$ in the final click reaction mix. Upon melamine embedding, the samples were cut and then imaged at a GSDIM setup (see Figure 3-19). The spots detected in each image were analyzed and three properties that were measured are: spot size, peak intensity, and summed intensity (see Figure 2-3 for details on how these parameters were obtained). 
no FP
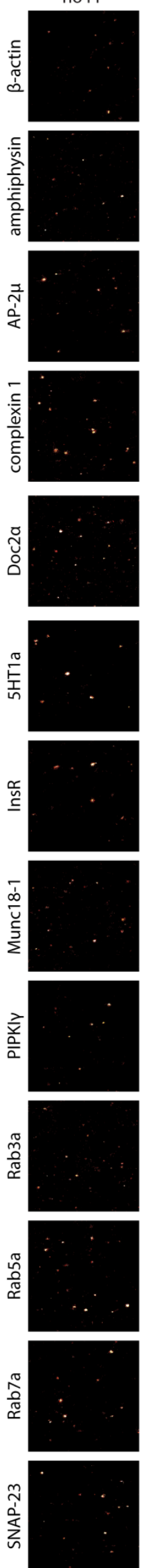

FP tagged spot size $(\mathrm{nm})$ peak intensity $(\mathrm{AU})$
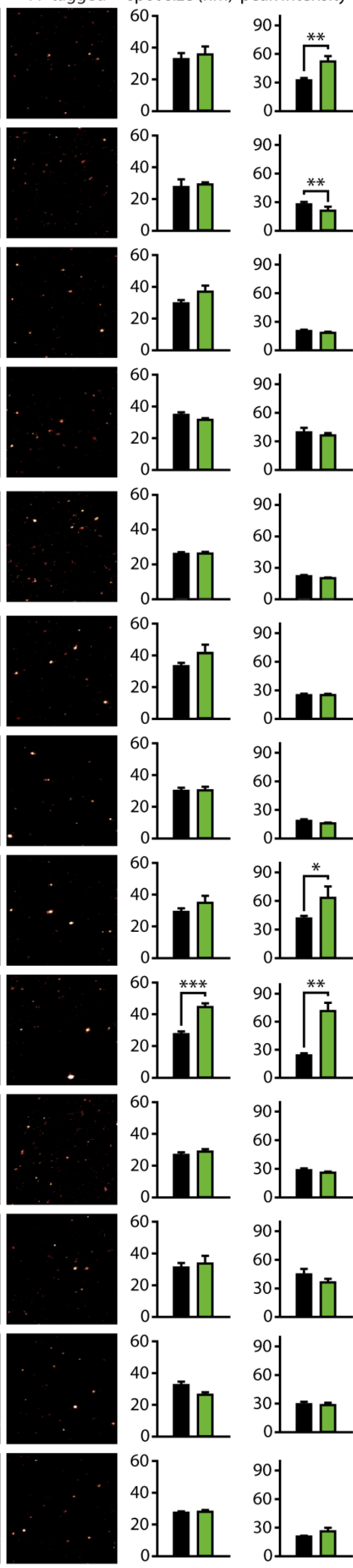

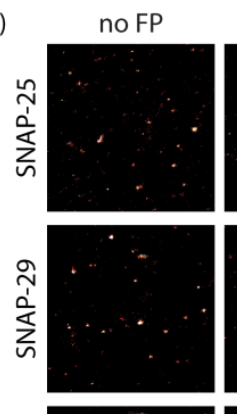

FP tagged spot size $(\mathrm{nm})$ peak intensity (AU)
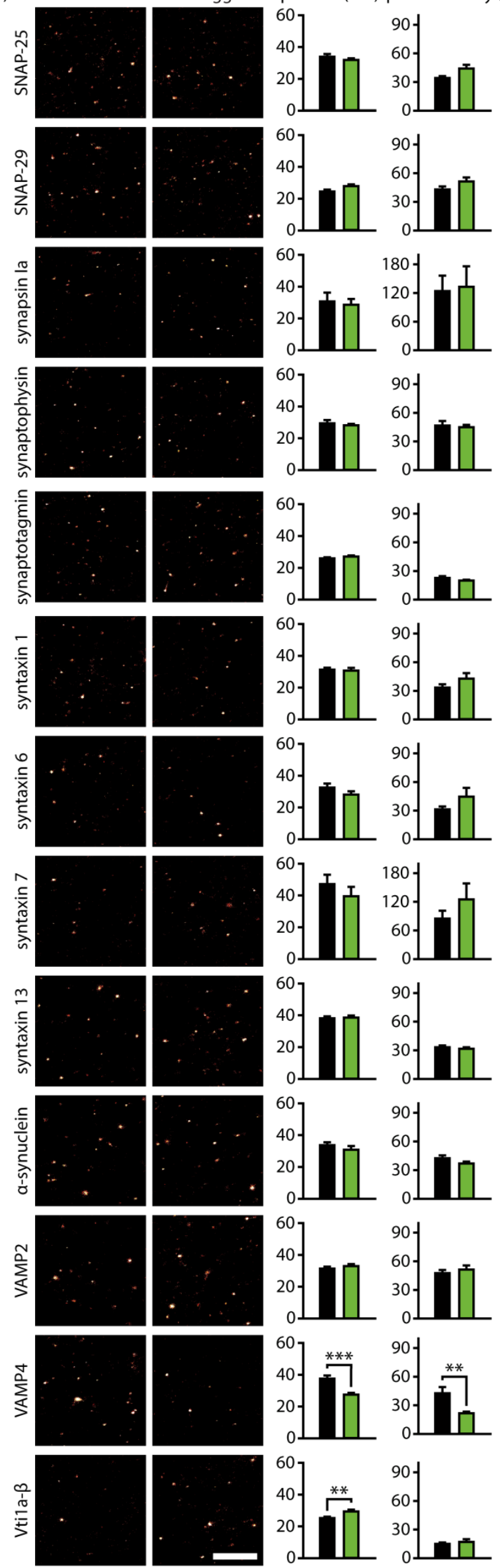


\section{Figure 3-19 The organization of investigated proteins in GSDIM}

The majority of the investigated proteins are not affected by the FP tags. Representative images Alexa647-azide ( $2 \mu \mathrm{M}$ final dilution) coupled via click chemistry to the proteins of interest without FP tags (no FP; left-hand panels) or FP tagged (right-hand panels) that have incorporated PRK into their primary structure. All samples consist of ultrathin sections $(100 \mathrm{~nm})$ of cells embedded in melamine and were imaged in GSDIM. Scale bar, $500 \mathrm{~nm}$. Data analysis was performed on the detected spots using Gaussian fits. For each protein, the means \pm SEM (on average from $\sim 100$ spots per protein) for the FWHM (spot sizes) and the peak intensities are represented in the bar graphs (no FP in black and FP tagged proteins in green). The statistical significance was assessed based on Student's t-test (P values: $\mathrm{P}<0.05 *, \mathrm{P}<0.01 * *, \mathrm{P}<0.001 * * *)$. Abbreviations: InsR - insulin receptor.

In order to look beyond the small spots (single molecule fluorescence data), which might mask significant information, data points above or below one standard deviation (1SD) were not considered for statistical analysis. So I considered these data points outliers and did not use them in the means depicted in Figure 3-20. The significance of the difference between the results obtained for FP chimeras and their non-tagged counterparts was assessed using Student's t-test. For the graphs in Figure 3-20 A-C, I analyzed three different properties of the spots: size, peak intensity as well as the summed intensity over each spot (total intensity). When spot size is considered (see Figure 3-20 A), there are three proteins for which I observed significant differences: PIPKI $\gamma$, VAMP4, and Vti1a- $\beta$. For Vti1a- $\beta$, the FP-tagged variant shows slightly increased protein cluster size (by cca $5 \mathrm{~nm}$ ) compared with the nontagged one. The same trend is noticeable for PIPKI $\gamma$, yet here the $20 \mathrm{~nm}$ difference is statistically highly significant. In contrast, VAMP4 forms bigger clusters in the absence of GFP by approximately $10 \mathrm{~nm}$ (highly significant results).

Five proteins showed significant differences in peak intensity values: $\beta$-actin, amphiphysin, Munc18-1, PIPKI $\gamma$, and VAMP4. The same proteins also exhibit significant differences in total spot intensities, while syntaxin 6 is the only protein for which the total intensity values are significantly different, but not the peak intensities. A possible reason for this, might be the higher sensitivity of the total intensity parameter in detecting dimmer spots.

The general trend for the proteins that show higher intensity values for the FP tagged protein than for the non-tagged variant (e.g. $\beta$-actin, Munc18-1, and PIPKI $\gamma$ ) is kept for both the peak and the total intensity values. The same applies for the proteins that form clusters that are less bright when FP tagged. 
A

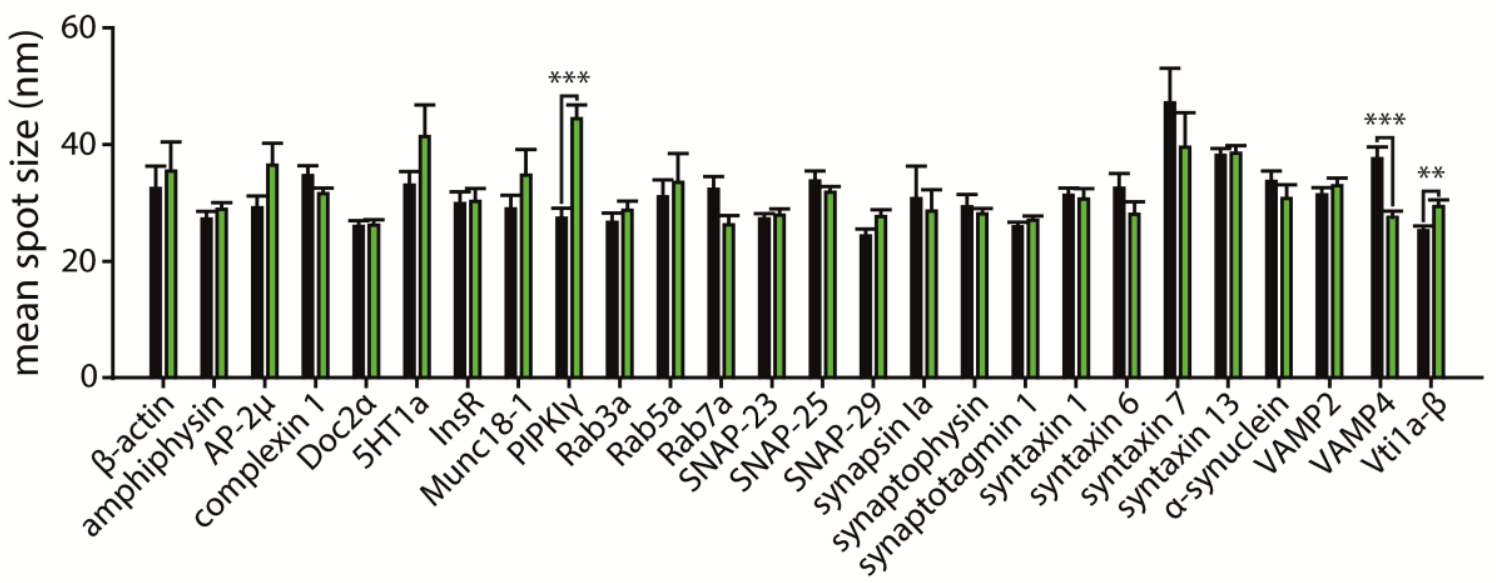

$\mathrm{B}$

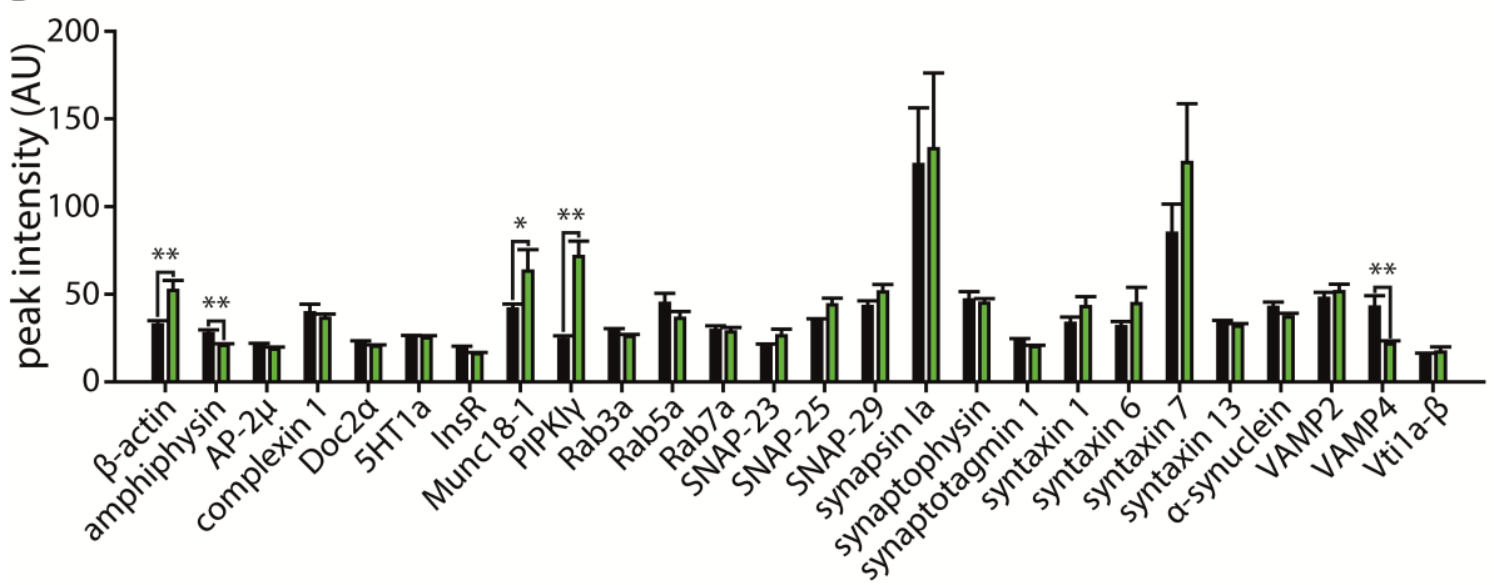

C

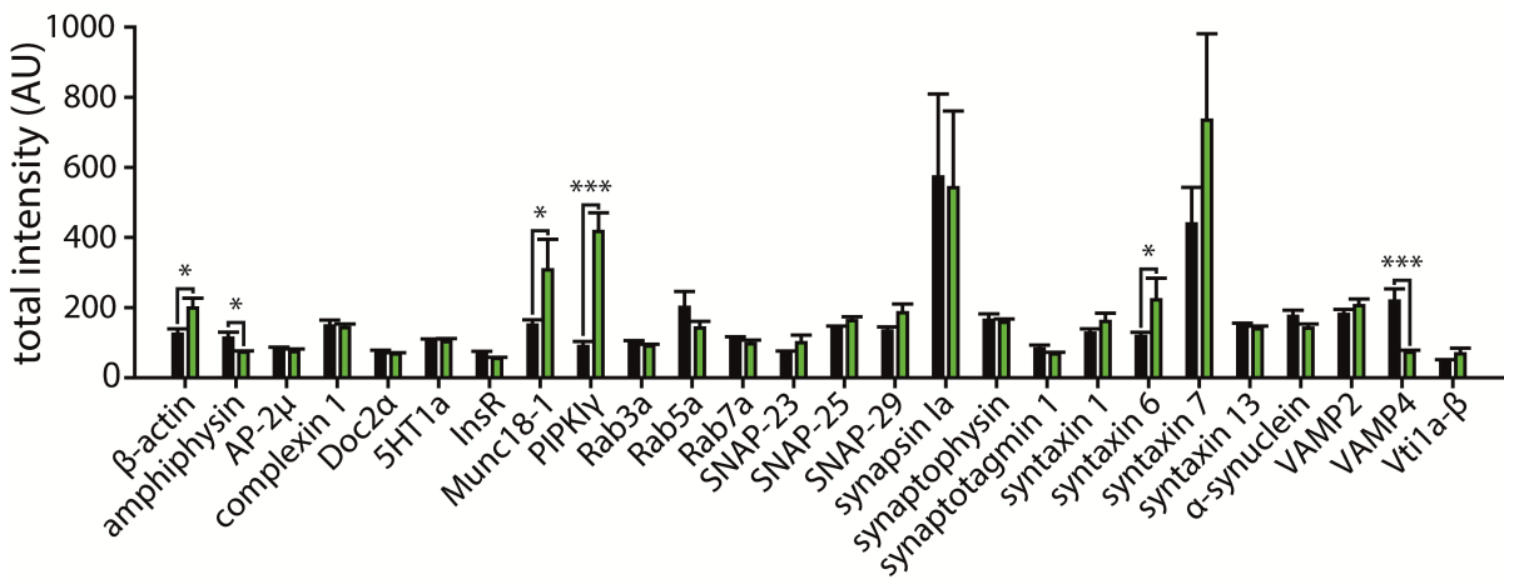

Figure 3-20 Comparison of mean spot size, peak and total spot intensity in GSDIM

Graphs depicting the size (A), peak (B) and total intensity $(\mathbf{C})$ of the spots detected for the 26 different proteins. The black bars represent non-tagged proteins, while green bars show their chimeric versions. $\mathrm{P}$ values in t-tests: $\mathrm{P}<0.05 *, \mathrm{P}<0.01 * *, \mathrm{P}<0.001 * * *$.

Another approach to systematize the results obtained for the non-tagged proteins and their FP-tagged counterparts is to look at their ratio while taking the non-tagged protein as a reference (see Figure 3-21 A and B). In Figure 3-21 A, the FP to no FP ratios for the spot 


\section{Results}

size and the peak intensity are shown for all the 26 proteins. The ratios for the majority of the investigated proteins are close to the baseline given by the no FP values.

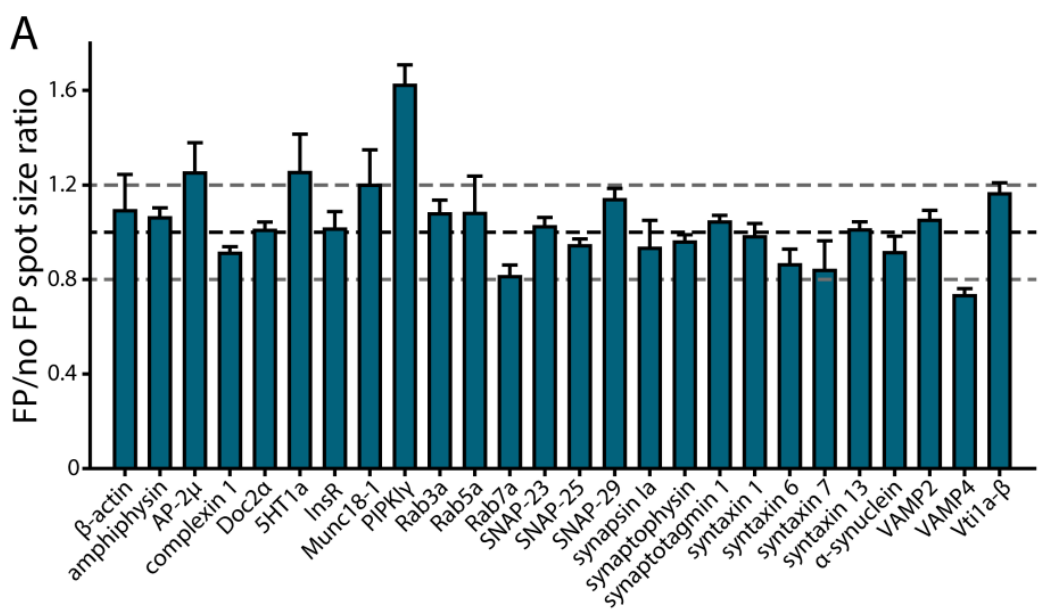

B
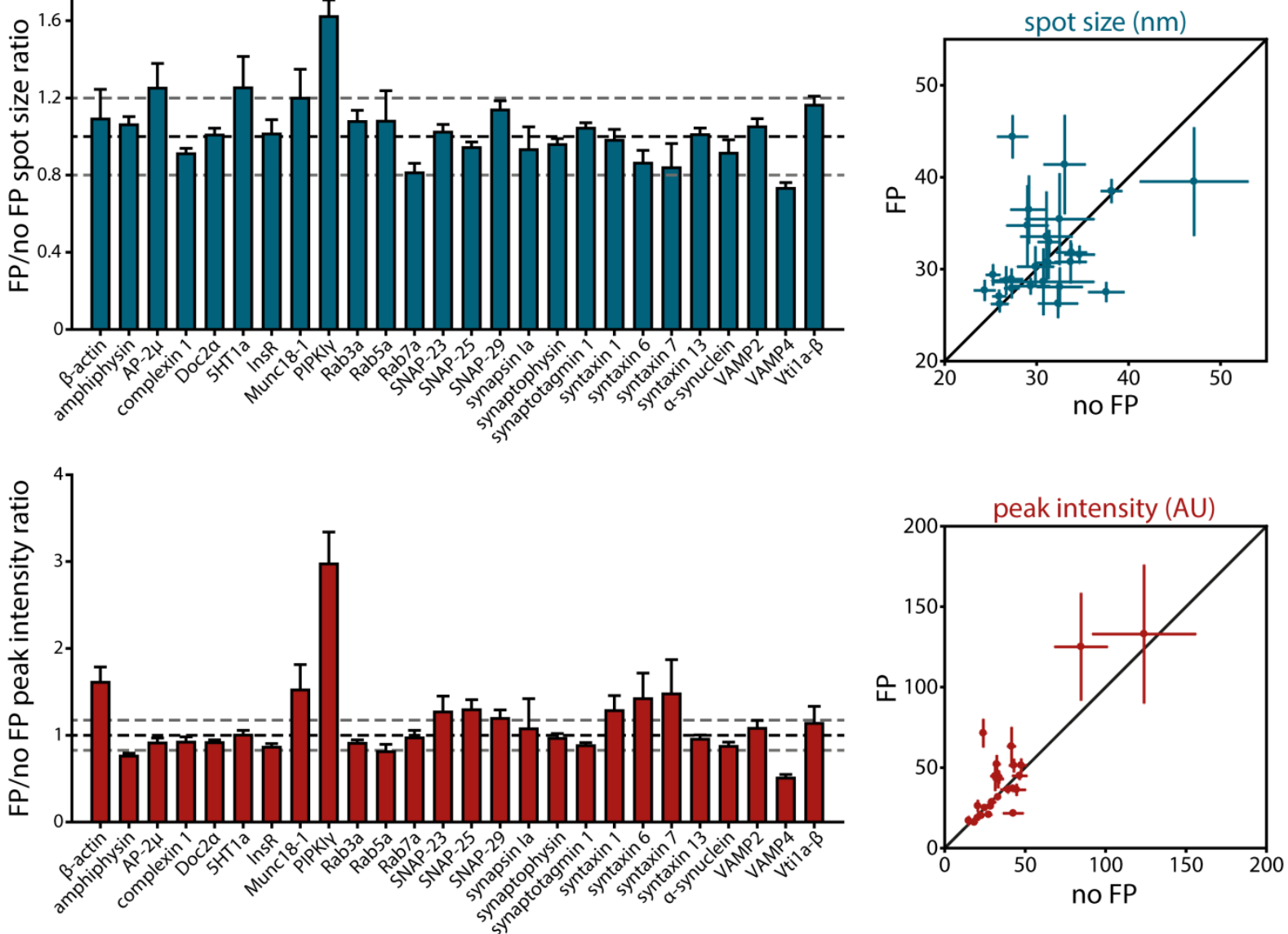

Figure 3-21 Graphical depictions of the ratios between the FP-tagged proteins versus the nontagged ones.

A. Bar plots showing the ratios of the FP-tagged protein over those without FP (no FP) for the spot sizes (upper panel, in blue green) and peak intensity (lower panel, in red). The black dashed line indicates the position of the no FP value (considered as 1), whereas the grey lines show the ratios with the values of 0.8 and 1.2, respectively. B. Graphs depicting the distribution of the FP-chimeras versus their non-tagged variants. Note that all data for spot size are represented in blue-green, while the data for peak intensity are shown in red. The diagonal lines indicate the position where the dots would be located if the values for the FP and no FP conditions would be identical.

However, except the proteins that have shown significant differences discussed above, there are a few others which have values differing by more than $20 \%$ above or below the baseline. For spot size measurements, the proteins are: AP-2 $\mu, 5 \mathrm{HT} 1 \mathrm{a}$, and Munc18-1, which are above the baseline, and only Rab7a is below the baseline. Note that for Vtila- $\beta$, even though the sizes of the clusters it forms in the presence or absence of an FP tag are significantly different, the difference is smaller than $20 \%$. For peak intensity ratios, there are six proteins (except the ones which showed statistically significant differences) that form clusters $20 \%$ 
brighter when FP tagged: SNAP-23, SNAP-25, and SNAP-29, as well as syntaxin 1, syntaxin 6 , and syntaxin 7 . Rab5a is the only protein chimera that forms clusters dimmer by $20 \%$.

In Figure 3-21 B, the distributions of the FP values for the spot size and peak intensity are shown as a function of the no FP values. The spot size values do not seem to correlate for the FP-tagged proteins versus their non-tagged counterparts, whereas there is a slight correlation for the peak intensities.

Next, I wanted to group the proteins based on all the parameters measured for the GSDIM data (see Figure 3-22). For this I used the $\mathrm{k}_{\text {means }}$ algorithm to partition the different proteins using the spot sizes, peak and total intensities as parameters (see Section 2.14.2 for more details). The two obvious outliers are VAMP4 and PIPKI $\gamma$ that form their own separate groups. These same proteins are the ones most affected by FP tagging: if the protein assemblies formed by PIPKI $\gamma$ increase in size and intensity when FP-tagged, in the case of VAMP4 the clusters are smaller and dimmer.

Interestingly, the main SNAREs involved in exocytosis or fusion with endosomes cluster together in the central region of the graph from Figure 3-22 (in blue), indicating that in their case there is little change upon FP tagging. The behavior of the other SNAREs - syntaxins 6, 7 and 13 - is somewhat different, and they group either with the largest assortment of proteins (syntaxin 13, see green data points in the graph from Figure 3-22) or with the smaller group formed together with $\beta$-actin and Munc18-1 (syntaxin 6 and syntaxin 7, red points from Figure 3-22). The green group consists of proteins that exhibit no major difference in intensity when FP tagged and contains almost all of the investigated cytosolic proteins and some of the membrane proteins. This indicates that soluble proteins (and some of the membrane proteins) are not significantly affected by FP tagging. In contrast, the red group shows higher intensity values than the blue and the green group. The proteins found here are either cytosolic ( $\beta$-actin and Munc18-1) and membrane-attached (syntaxin 6 and syntaxin 7). This analysis further strengthens the conclusion that FPs do not induce major changes in the organization of protein assemblies. 
$\mathrm{k}_{\text {means }}$ clusters by size, peak and total intensity

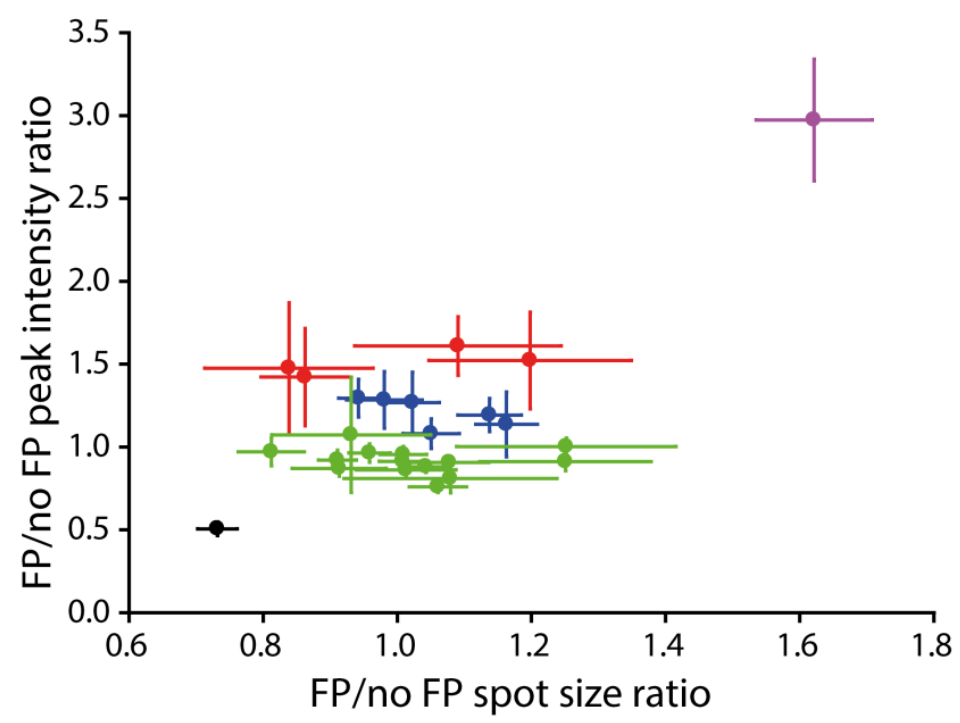

- $\quad \beta$-actin, Munc18-1, syntaxin 6, syntaxin 7

- SNAP-23, SNAP-25, SNAP-29, syntaxin 1, VAMP2, Vti1a- $\beta$

- VAMP4

- PIPKIY

- AMPH, AP2- $\mu$, complexin 1, Doc2a, 5HT1a, InsR, Rab3a, Rab5a, Rab7a, synapsin la, synaptophysin, synaptotagmin I, syntaxin 13 , a-synuclein

\section{Figure 3-22 $\mathbf{k}_{\text {means }}$ cluster analysis}

The 26 investigated proteins were grouped using the spot size, peak and total intensity as parameters. The proteins which clustered (or grouped) together are shown in the same colors and their identities are indicated in the figure legend. 


\subsection{Development of SPILL}

NanoSIMS offers the possibility to investigate many aspects of cell composition and metabolism because it can detect in parallel 7 different isotopic species on a routine basis. It also achieves a good resolution: up to $30 \mathrm{~nm}$ in the $\mathrm{xy}$ plane and up to $1-2 \mathrm{~nm}$ in the $\mathrm{z}$ axis (Lechene et al., 2006). NanoSIMS is becoming increasingly used in biology. But so far proteins have been labeled for this technique using isotopically marked canonical amino acids. These are incorporated in all proteins, so this approach provides a general protein labeling and, at the same time, allows for protein turnover studies (Lechene et al., 2006). Specific proteins have been revealed with the help of antibodies linked to heavy metal ions (Angelo et al., 2014), which present their own disadvantages (see discussion), or through correlative approaches (Saka et al., 2014b). At the moment there is no method available to genetically encode labels for NanoSIMS in an analogous manner to FP tagging in fluorescence microscopy.

In order to take advantage of the insight into cell metabolism offered by NanoSIMS, I established a method to specifically labeling proteins for their visualization using this technique. In this section, I describe a new technique for specific protein isotopic and fluorescence labeling, in short SPILL, which is based on the incorporation of the an ncAA followed by click reaction with probes containing both isotopic markers for NanoSIMS and fluorescence microscopy (see Figure 3-23).

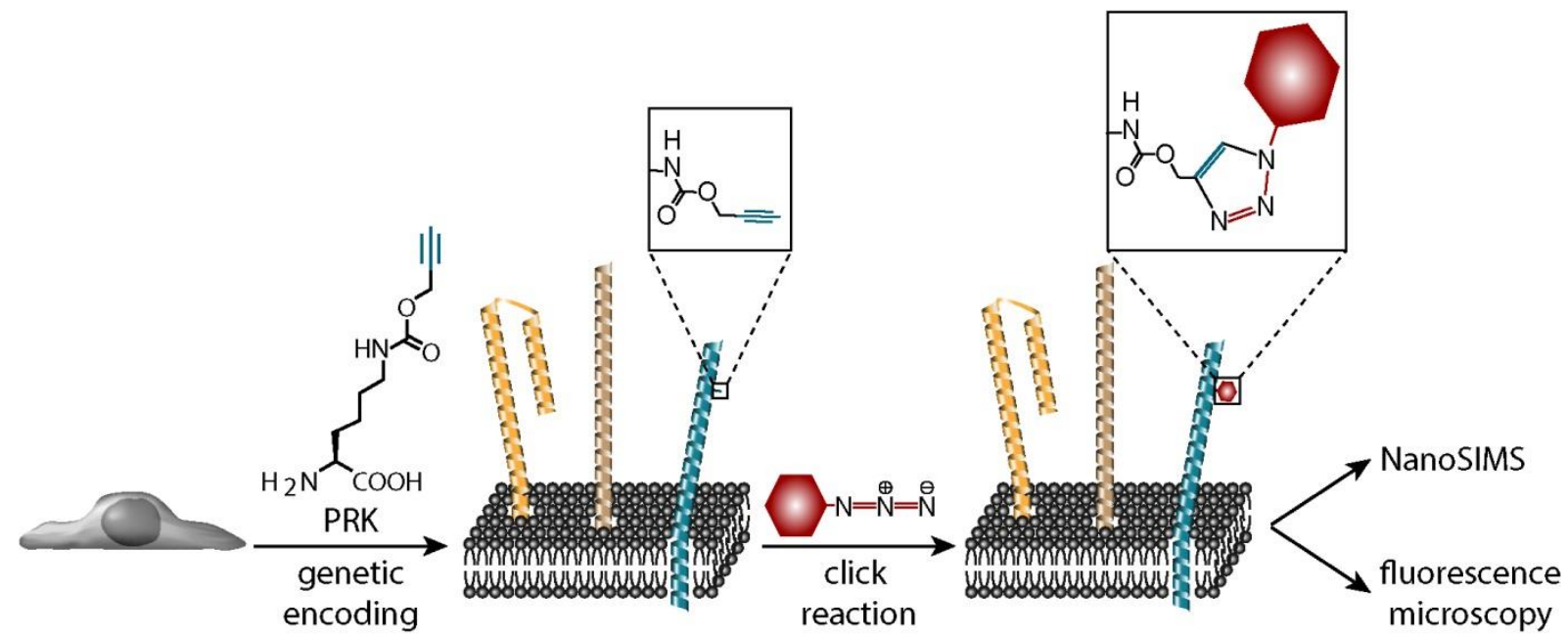

Figure 3-23 The specific protein issotopic and fluorescence labeling (SPILL) technique

SPILL involves the incorporation of PRK into the protein of interest followed by click reaction (upon fixation and permeabilization) with a dual probe for NanoSIMS and fluorescence microscopy. 
In principle, any ncAA can be used for this purpose as long as its incorporation and reaction with the SPILL probe has been optimized. In this study, I have employed propargyl-L-lysine (PRK), the non-canonical amino acid that performed best in labeling the proteins of interest. Aside from investigating a specific protein of interest, many other cell constituents can be isotopically labeled (see below).

\subsubsection{Testing Different Probes for Metabolic Investigations}

I tested different peptide backbones as a support for the fluorescent moiety and the part enriched in isotopes that can be detected in NanoSIMS. The probes with different backbones are: TriazNF1 (negatively charged peptide), TriazNF2 (non-charged peptide), and TriazNF3 (positively charged peptide). The most successful one was the negatively charged one and it showed the least nonspecific binding in both the control and non-transfected cells (see Figure 3-24 A). Both the neutral and the positively charged peptide, in contrast, showed a very strong tendency to nonspecifically bind cytosolic components in all the samples (Figure 3-24 B-C). This resulted in a high background that made it impossible to establish if the probe efficiently labels the proteins that have incorporated PRK. Remarkably, the positively charged peptide (TriazNF3) creates a higher background than the neutral peptide (TriazNF2). This might be due to the nonspecific binding to the negatively charged species present in the cells, such as the phospholipids (e.g. phosphatidylserine and phosphatidylinositol) or nucleic acids such as RNA. The chemical structure of TriazNF1 can be seen in Figure 3-25 A, whereas for chemical formulas of TriazNF2 and TriazNF3 refer to the Appendix.

Hence, I selected the negatively charged peptide backbone as the most suitable for NanoSIMS investigations. Together with the group of Prof. Ulf Diederichsen, we designed and tested two probes for SPILL: TriazNF1, which is enriched in ${ }^{15} \mathrm{~N}$, and SK155, which has a high ${ }^{19} \mathrm{~F}$ content (see Figure 3-25; Kabatas et al., 2015; Vreja et al., 2015). SK155 is also specific in labeling the proteins of interest for SPILL (Figure 3-26).

For these SPILL probes we used the Star635 dye, and not the KK114 or the Star635P, because the Star635-NHS ester is the most stable (Kolmakov et al., 2012). In addition, compared with KK114 and Star635P, Star635 has a longer linker (Kolmakov et al., 2012) which could improve the reactivity of the final probe by allowing steric freedom to the azide group (see Figure 3-25). Therefore, this ester derivative was used for the synthesis of both NanoSIMS probes, SK155 and TriazNF1. 


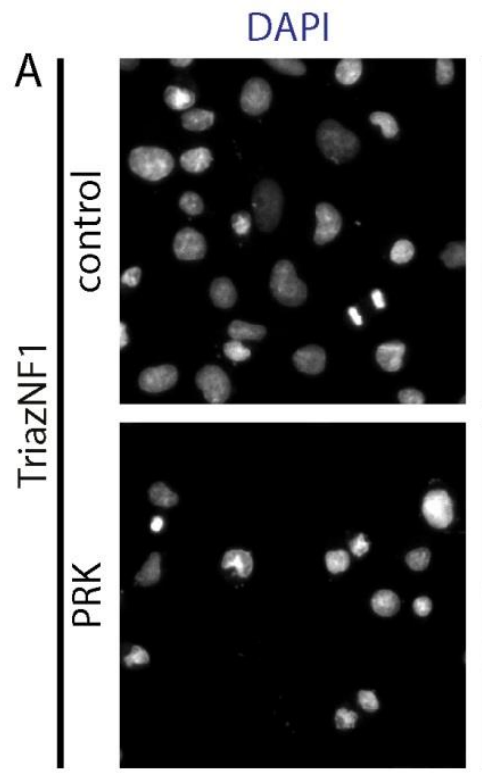

B
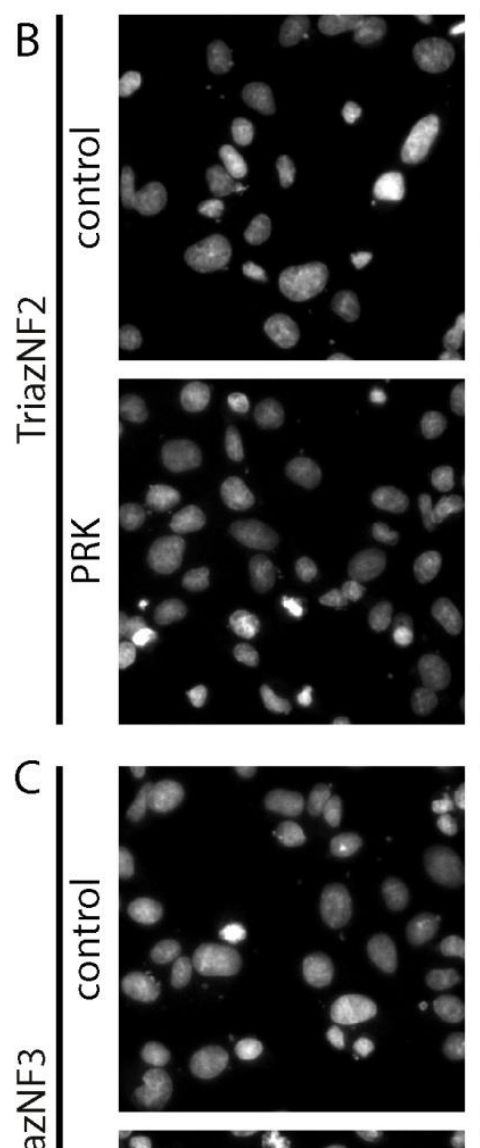

学

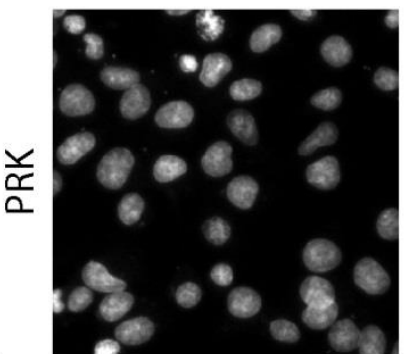

SNAP-25-GFP

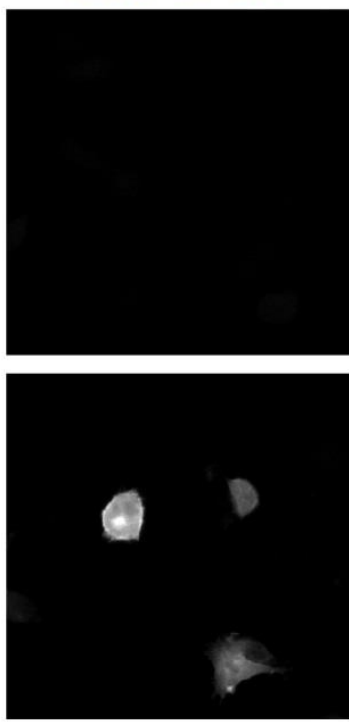

click signal
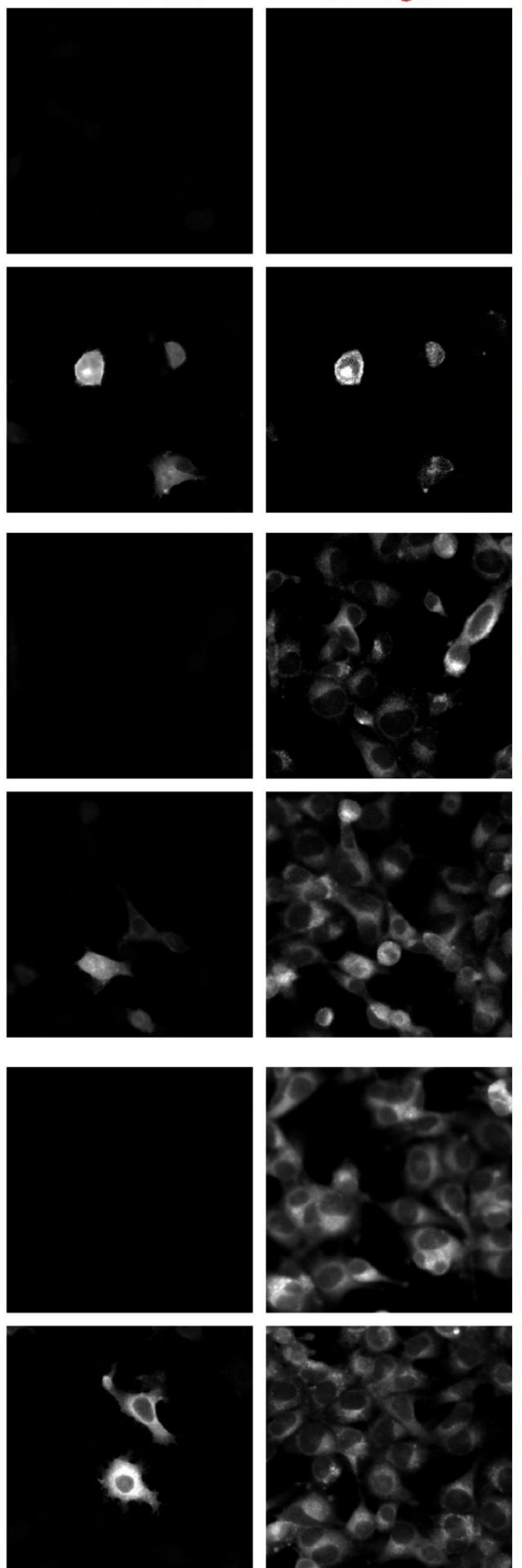
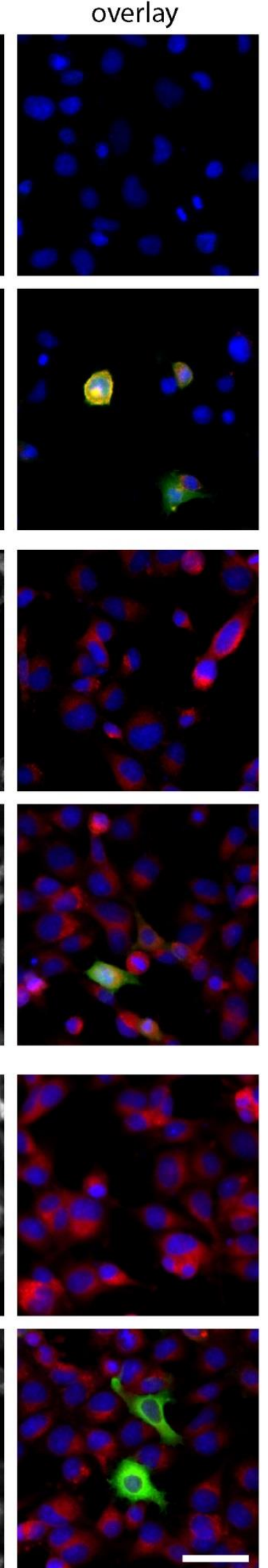

Figure 3-24 Specificity of different high-content nitrogen compounds in click reactions 


\section{Figure 3-24 Specificity of different high-content nitrogen compounds in click reactions}

BHK cells were allowed to incorporate PRK into SNAP-25-GFP F84TAG for 18 hours. The cells were then fixed, permeabilized and labeled with $7 \mu \mathrm{M}$ Star635-containing peptides TriazNF1 (A; acidic), TriazNF2 (B; neutral), and TriazNF3 (C; basic). Note that the images are identically scaled for the GFP and the DAPI channels. However, the Star635 fluorescence intensity is too different (i.e. very high for the samples with high background, especially TriazNF3) to allow for equal scaling. Scale bar, $40 \mu \mathrm{m}$.

A

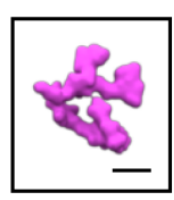

$\operatorname{Lin}_{15}$<smiles>CCCNc1nc(NCC)nc(NCC)n1</smiles>

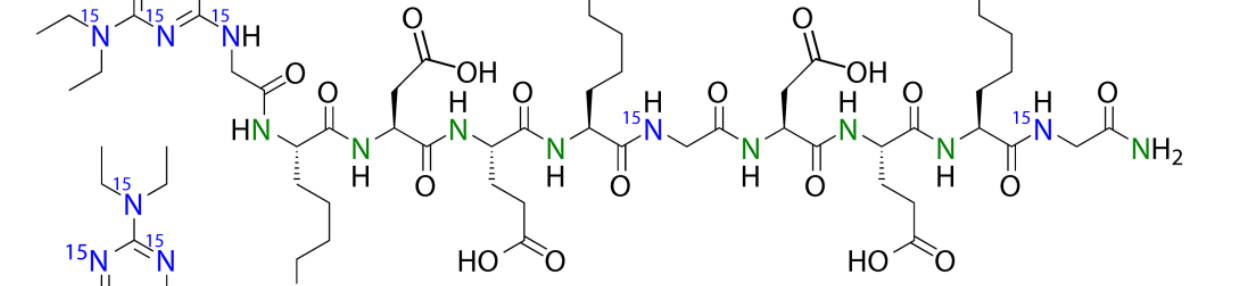<smiles>CCN(CC)c1ncnc(NC(=O)NCCI)n1</smiles><smiles>CC1(C)C=C(CO)c2ccc(O)c3c2N1CCC3</smiles><smiles>CCCN(CO)C(=O)c1c(F)c(F)c(SCCS(=O)(=O)[O-])c(F)c1-c1c2cc3c4c(c(C)cc3c1O)CCC[N+]4(C)CCC2</smiles>
$\mathrm{OH}$

\section{TriazNF1}

B

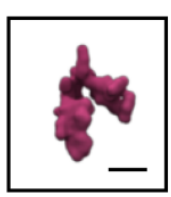<smiles>NC(=O)CC(NC(=O)[C@H](CCCCNC(=O)c1c(F)c(F)c(F)c(F)c1F)NC(=O)c1c(F)c(F)c(F)c(F)c1F)C(=O)O</smiles>

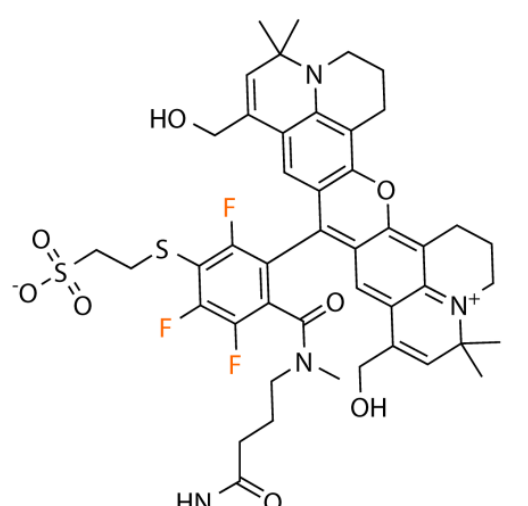




\section{Figure 3-25 Probes for SPILL labeling: molecular structures of TriazNF1 and SK155}

Both molecules contain a nonapeptide peptide backbone with acidic side chains (peptide sequence: $\mathrm{H}_{2} \mathrm{~N}$-Gly-Lys-Glu-Asp-Gly-Lys( $\left.\mathrm{N}_{3}\right)$-Asp-Lys), an azide group for click reaction, a Star635 fluorescent moiety, and groups for specific isotope enrichment. Note that the lysines are used for the attachment of the fluorophore and the istopic markers, so they do not contribute to the charge of the probes. The molecules in the insets represent 3D renderings of the two probes. Scale bars for the insets, $1 \mathrm{~nm}$. A. TriazNF1 contains $14 \times{ }^{15} \mathrm{~N}$ (in blue) and $12 \times{ }^{14} \mathrm{~N}$ (just $\mathrm{N}$ here; in green), as well as 3 fluorines $\left({ }^{19} \mathrm{~F}\right.$ or just F; in orange). B. SK155 contains $13 \times{ }^{19} \mathrm{~F}$. The nitrogen atoms not highlighted because they are of no importance for the isotopic labeling using this probe. Both compounds were synthesized by Selda Kabatas.
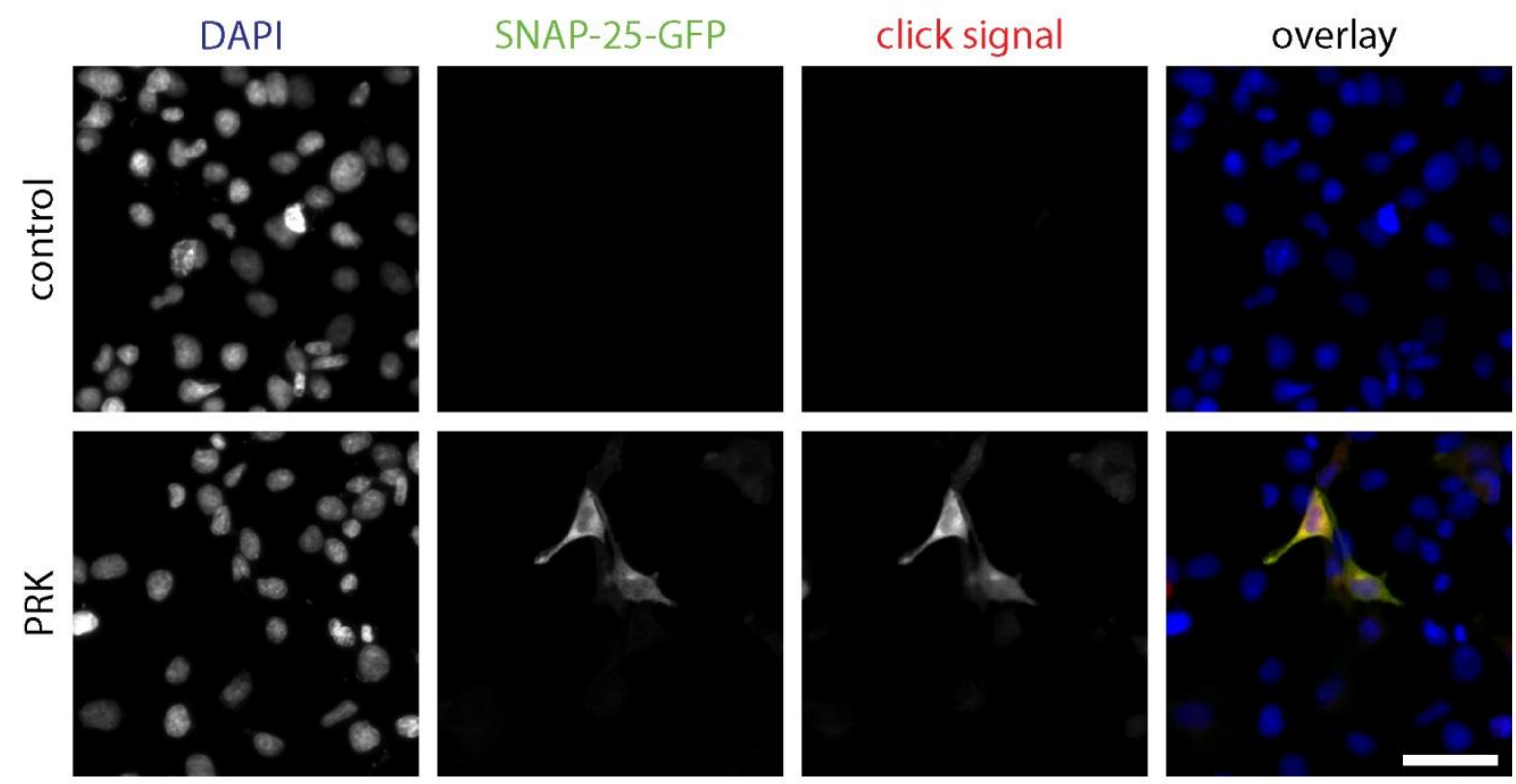

\section{Figure 3-26 Specificity of SK155 in SPILL}

BHK expressing SNAP-25-GFP F84TAG in the presence of PRK or in its absence (control) were fixed, permeabilized, and click labeled with $7 \mu \mathrm{M}$ SK155. Note that the images are identically scaled in the DAPI, GFP, and Star635 channels, respectively. Scale bar, $40 \mu \mathrm{m}$.

NanoSIMS measurements involve inserting the samples in high vacuum and sputtering them with high-energy ion beams to release secondary ions. For this, the samples require thorough fixation: I employed two fixation steps with $0.2 \%$ glutaraldehyde- $4 \%$ PFA, before permeabilization and then after all the stainings were over. In addition, the samples should also be embedded in a plastic resin to preserve their structure in high vacuum and during the ion bombardment. I embedded the samples in LR white resin and placed them after thinsectioning on silicon wafers. These wafers enable NanoSIMS due to their conductive surface (see Figure 3-27 A), as well as fluorescence imaging. 


\section{Results}

I first identified the cells expressing the proteins of interest in fluorescence microscopy and marked the regions of interest for easily finding them at the NanoSIMS device (Figure 3-27 B-C). Notice that in Figure 3-27 C there is only one transfected cell (i.e. visible in the Star635P fluorescence), while the other two cells do not express the protein of interest (in this case syntaxin 1$)$.
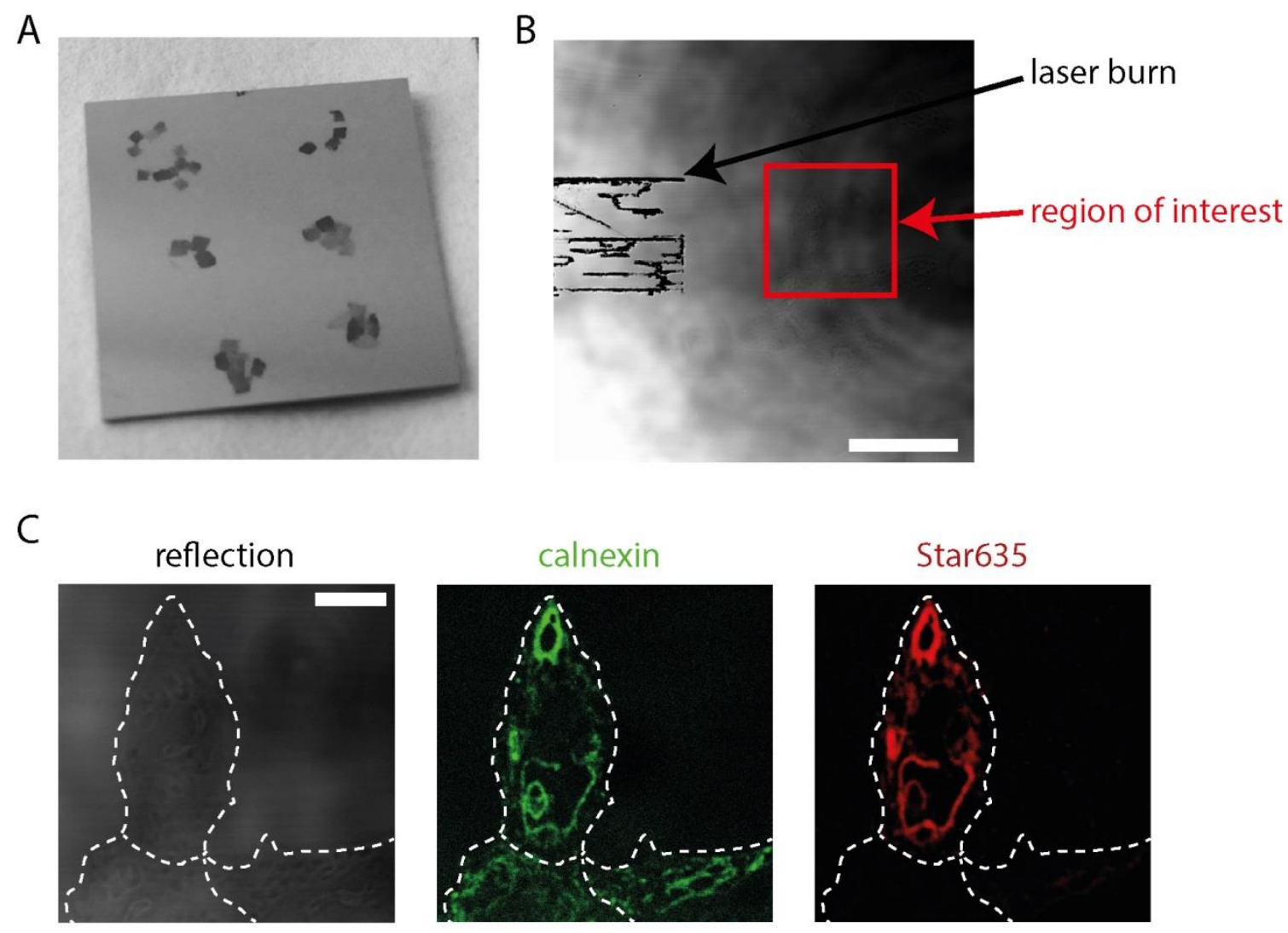

\section{Figure 3-27 Sample preparation for confocal and NanoSIMS imaging}

Sample preparation for imaging at the confocal microscope and at the NanoSIMS involves placing sections of the embedded samples on a silicone wafer. The samples consist of BHK cells that have incorporated PRK and are subsequently labeled with SK155 and then embedded in LR White resin. A. Thin sample sections are deposited on a silicone wafer for NanoSIMS imaging. B-C. The samples are first imaged using confocal microscopy and the regions of interest are marked using a multiphoton laser. The resulting laser burn present on the wafer is unique and will later help identify the region of interest in secondary ion mass spectrometry imaging. The region of interest $(\mathbf{C})$ contains a transfected cell that has incorporated the propargyl-L-lysine in syntaxin 1. This was subsequently labeled with SK155, a marker for NanoSIMS that also contains a Star635 fluorophore (shown in red). In the same region, two other cells (non-transfected) are present that do not show SK155 labeling but can be distinguished by their calnexin immunostaining (shown in green). Scale bars, $20 \mu \mathrm{m}$ and $5 \mu \mathrm{m}$, respectively. 


\subsubsection{Correlation between NanoSIMS Measurements and Confocal Imaging}

\section{TriazNF1}

The element nitrogen naturally found on Earth was formed in the carbon-nitrogen-oxygen cycle in stars and consists of two stable isotopes: ${ }^{14} \mathrm{~N}$ in a percent natural abundance of $99.632 \%$ (i.e. \% of atoms) and, to a smaller extent, ${ }^{15} \mathrm{~N}$, only $0.368 \%$ (Rosman and Taylor, 1998). Both of these isotopes are taken up by nitrogen-fixing bacteria and spread throughout the entire living regnum at a relatively constant molar ratio of 0.00367 (see discussion below). The natural abundance of ${ }^{15} \mathrm{~N}$ is too low to raise any issues for general metabolic labeling techniques that heavily rely on ${ }^{15} \mathrm{~N}$ as an isotopic marker, especially for the labeling of nuleic acids and proteins which are major constituents of living matter. In contrast, the presence of ${ }^{15} \mathrm{~N}$ poses problems in terms of background only if a small fraction of the biological components in the sample are targeted for labeling This is the case when one protein of interest is investigated. In order to circumvent this issue, I designed a molecule highly enriched in the ${ }^{15} \mathrm{~N}$ isotope: TriazNF1 (see Figure 3-25 A).

In the previous chapter, I showed that TriazNF1 specifically labels proteins for fluorescence imaging (see Figure 3-24). Here I employed TriazNF1 to specifically reveal the proteins of interest in NanoSIMS. No specific labeling is detected in the non-transfected cell, shown here as a control (compare the two cells shown in Figure 3-28 A and B). Due to its chemical properties, nitrogen cannot be detected alone in NanoSIMS measurements, but only as cyanide ions, ${ }^{12} \mathrm{C}^{14} \mathrm{~N}^{-}$or ${ }^{12} \mathrm{C}^{15} \mathrm{~N}^{-}$(referred herein simply as ${ }^{14} \mathrm{~N}$ or ${ }^{15} \mathrm{~N}$ ). The background from the naturally occurring ${ }^{15} \mathrm{~N}$ isotope clearly precludes to a high extent the visualization of the specific signal coming from the protein of interest (in this case syntaxin 1) labeled with TriazNF1. Compare the ${ }^{15} \mathrm{~N}$ panel with the Star635 panel in Figure 3-28 B. In contrast, using the ${ }^{15} \mathrm{~N}$ to ${ }^{14} \mathrm{~N}$ ratio, the correlation of the signal becomes apparent. Unexpectedly, fluorine $\left({ }^{19} \mathrm{~F}\right)$, a monoisotopic element that is present in only 3 atoms per TriazNF1 molecule, also showed specific signal to some extent. This prompted further improvements in the design of the SPILL probe and lead to the SK155 molecule (see below).

The cells were also fed with ${ }^{13} \mathrm{C}$-marked leucine for three days in order to label the newly synthesized proteins. Neither the ${ }^{13} \mathrm{C}$ measurement (data not shown) nor its ratio to ${ }^{12} \mathrm{C}$ (Figure 3-28 B, lower panel) did not reveal any significant information due to the unsatisfactory signal-to-noise ratio. This is a result of the elevated natural abundance of ${ }^{13} \mathrm{C}$. 


\section{Results}

This isotope constitutes $1.07 \%$ of the total carbon atoms found on Earth (Rosman and Taylor, 1998). Hence, if any correlations with protein metabolism are desired for the protein of interest, then the ${ }^{15} \mathrm{~N}$ to ${ }^{14} \mathrm{~N}$ ratio should be employed. Figure 3-28 $\mathrm{B}$ (in the last image on the lower row) also exemplifies the possibility of combining TriazNF1 with immunostainings, in fluorescence microscopy (the endoplasmic reticulum protein calnexin is shown).

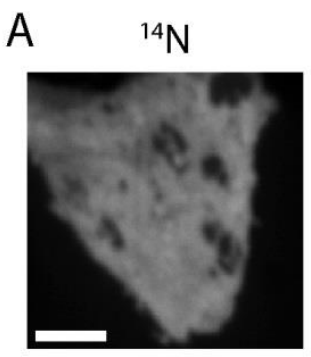

B Star635
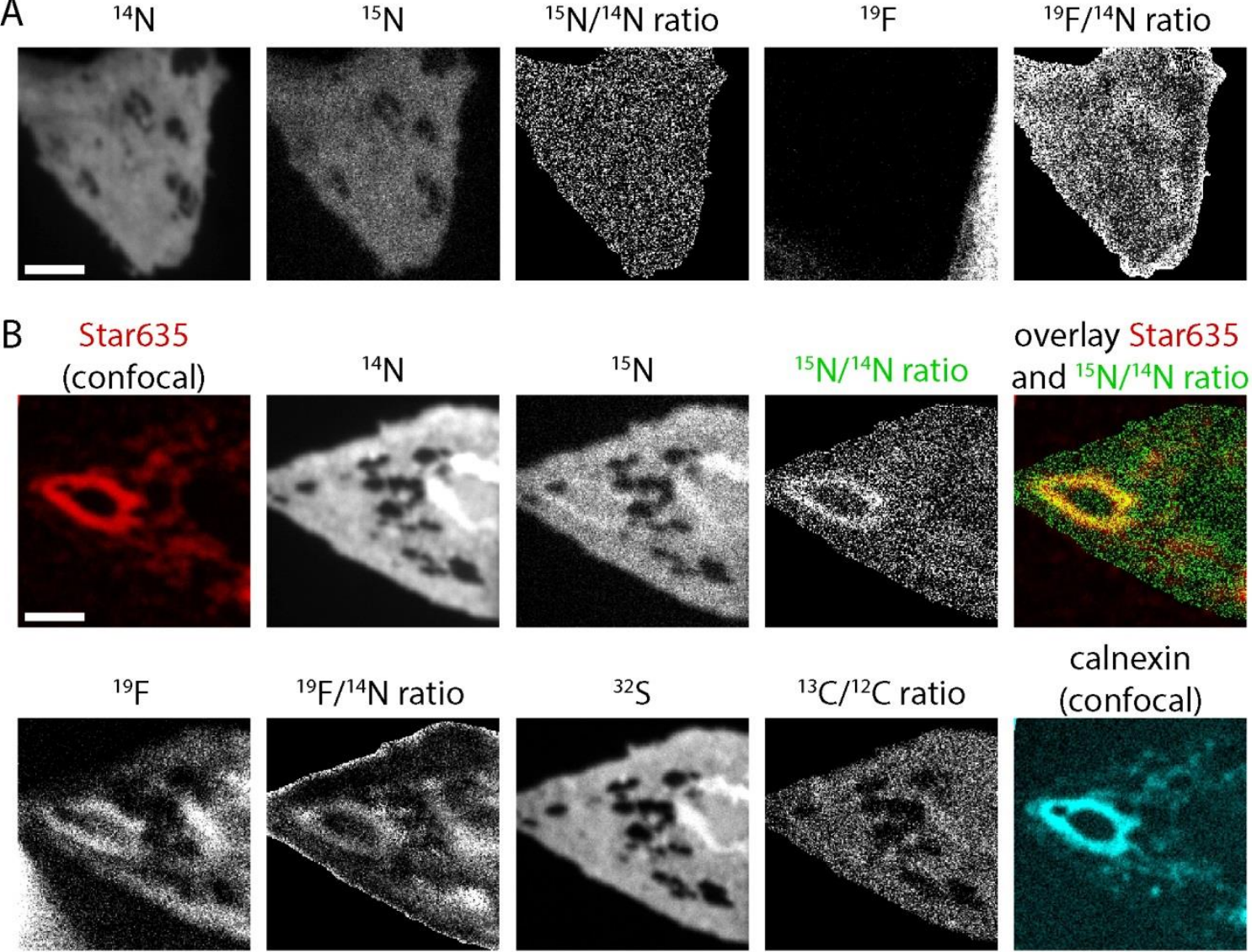
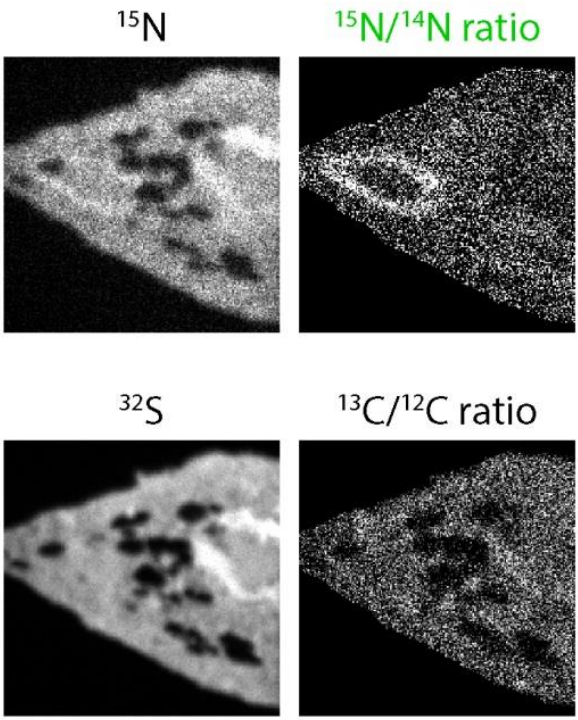

overlay Star635 and ${ }^{15} \mathrm{~N} /{ }^{14} \mathrm{~N}$ ratio

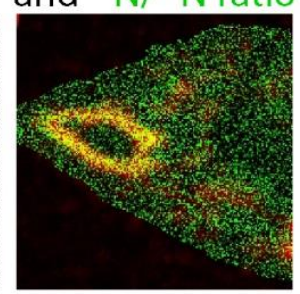

calnexin (confocal)

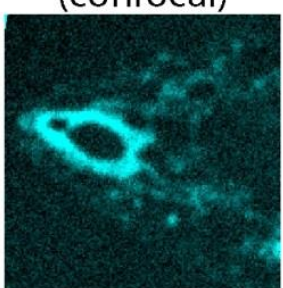

Figure 3-28 TriazNF1 specificity in NanoSIMS and confocal imaging

The probe TriazNF1 specifically labels proteins for visualization in NanoSIMS and fluorescence imaging. The genetic encoding of PRK into syntaxin 1 (V255TAG) as well as incorporation of Lleucine-2- ${ }^{13} \mathrm{C}$ into newly secreted proteins was performed in BHK cells. The samples were labeled with TriazNF1, embeded in LR White, and thin-sectioned. A-B. Representative images of a nontransfected cell (A) or a cell expressing syntaxin 1 labeled with TriazNF1 (B). Note that the corresponding images for the transfected and non-transfected cell are identically scaled. B. In the top panels the confocal microscopy image of TriazNF1 (given by the Star635 fluophore) and the NanoSIMS measurements for ${ }^{14} \mathrm{~N}$ and ${ }^{15} \mathrm{~N}$ isotopes, their ratio, as well as an overlay of Star635 fluorescence with the ${ }^{14} \mathrm{~N}$ to ${ }^{15} \mathrm{~N}$ ratio image. More images of the same cell are shown below: ${ }^{19} \mathrm{~F}$ and its ratio with ${ }^{14} \mathrm{~N},{ }^{32} \mathrm{~S}$, and the ratio of ${ }^{13} \mathrm{C}$ to ${ }^{12} \mathrm{C}$ for NanoSIMS or the endoplasmic reticulum marker calnexin in confocal (shown in cyan). The ratio images present a mask surrounding the cell. Scale bar for all images, $2 \mu \mathrm{m}$. 
Based on the ${ }^{15} \mathrm{~N}$ to ${ }^{14} \mathrm{~N}$ ratio, TriazNF1 specifically reveals the proteins of interest in transfected and labeled cells, but not in the non-transfected ones (compare bar plots in Figure 3-29 A). The ${ }^{14} \mathrm{~N} /{ }^{15} \mathrm{~N}$ ratio in non-transfected cells is somewhat higher than the natural abundance ratio. This might be due to the variation of the natural abundance in the sample. For instance, it has been shown that the ${ }^{15} \mathrm{~N}$ to ${ }^{14} \mathrm{~N}$ ratio varies along the trophic chains, with organisms on top of the food chain having higher levels of ${ }^{15} \mathrm{~N}$ (by 3-4\%o at each trophic level; Adams and Sterner, 2000). In addition an enrichment in ${ }^{15} \mathrm{~N}$ has been observed for the plants and animals from arid ecosystems (Szpak et al., 2013).

The presence of the two main nitrogen isotopes in the structure of TriazNF1 at a known ratio can be used to calculate the relative TriazNF1 copy numbers from a system of equations in which the ${ }^{15} \mathrm{~N}$ and ${ }^{14} \mathrm{~N}$ counts as well as their natural abundance ratio are taken into account (for details on these calculation see Section 2.15). Hence the copy numbers in which the protein of interest is found in the sample can be estimated in each voxel (see Figure 3-30 B). What is more, this measurement is insensitive to background signals.
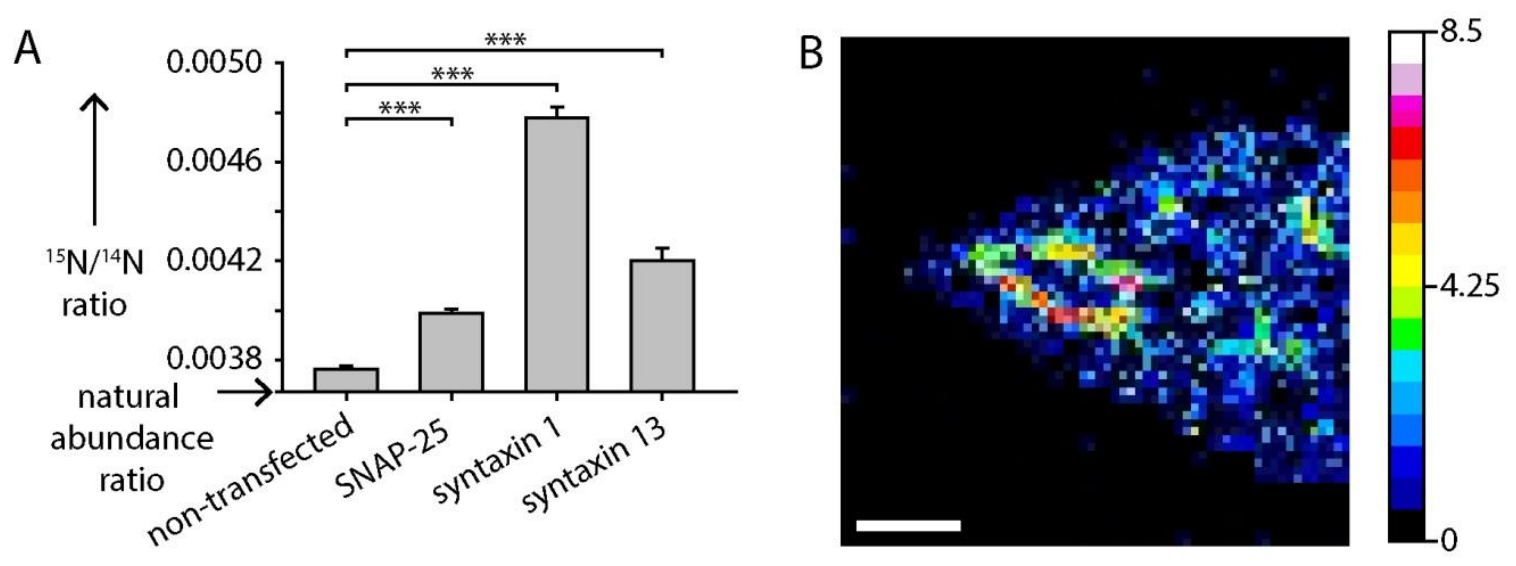

Figure 3-29 Specificity and quantitation of the TriazNF1 signal in NanoSIMS

A. The mean ${ }^{15} \mathrm{~N} /{ }^{14} \mathrm{~N}$ ratios for cells expressing different SNARE proteins are significantly higher (***, $\mathrm{P}<0.001$ compared to control in Student's t-test) than the ratio for non-transfected cells. The $\mathrm{y}$ axis starts at the value of the natural abundance ratio (0.00367). For each condition, I analyzed a number of circular cellular regions of interest, of $\sim 0.123 \mu \mathrm{m}^{2}: 15$ regions for SNAP-25, 54 for syntaxin 1, 31 for syntaxin 13, and 137 for non-transfected cells. The error bars indicate the standard errors. (B). Image depicting syntaxin 1 copy number inferred from the ${ }^{15} \mathrm{~N} /{ }^{14} \mathrm{~N}$ ratio. Scale bar, $2 \mu \mathrm{m}$. 


\section{Results}

\section{SK155}

As a next step in this project, I wanted to obtain a better contrast in the NanoSIMS images. My main aim was to find better isotopic markers that enable turnover investigations at the same time. The criteria for such a marker are: i) it should have a very low abundance in biological samples, ii) it is well ionized and detected under SIMS conditions, iii) few other metabolic compounds should employ it for turnover studies, and iv) relatively inexpensive and easily available. With these goals in mind, the best candidate proved to be the monoisotopic element fluorine that exists in nature only as ${ }^{19} \mathrm{~F}$ (Rosman and Taylor, 1998). As a result, ${ }^{19} \mathrm{~F}$ does not require expensive isotopic enrichment procedures and its derivatives are easily available. In addition, it has only been marginally used for metabolic investigations compared with ${ }^{15} \mathrm{~N}$.

${ }^{19} \mathrm{~F}$ has already been detected in the TriazNF1 samples (see Figure 3-28), yet contrast was not satisfactory. To obtain a better signal-to-noise for the ${ }^{19} \mathrm{~F}$ measurements, the SK155 probe was designed to have a number of fluorine atoms increased to 13 (from 3 in TriazNF1; Figure 3-25). The SK155 specificity in labeling the proteins for fluorescence microscopy was already shown (see Figure 3-26). In Figure 3-30, I exemplify the correlation between the signal in NanoSIMS and in confocal microscopy for the three proteins included in this study: syntaxin 1 (Figure 3-29 A), SNAP-25 and syntaxin 13 (Figure 3-29 B). The overlays of the ${ }^{19} \mathrm{~F}$ image with the SK155 (Star635) fluorescence image in Figure 3-29 confirm the good colocalization of the two signals. For syntaxin 1 , the additional ${ }^{14} \mathrm{~N}$ NanoSIMS as well as calnexin staining confocal images are included. The specificity of the labeling is attested by the ratio between ${ }^{19} \mathrm{~F}$ and ${ }^{14} \mathrm{~N}$ (see Figure $3-30$ ). I measured this ratio in non-transfected cells as well as in the cells expressing the different proteins. Their comparison revealed statistically significant results for the cells expressing the proteins of interest. 
A Star635

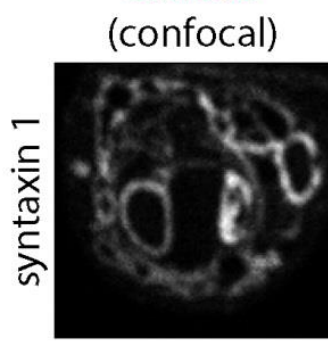

B
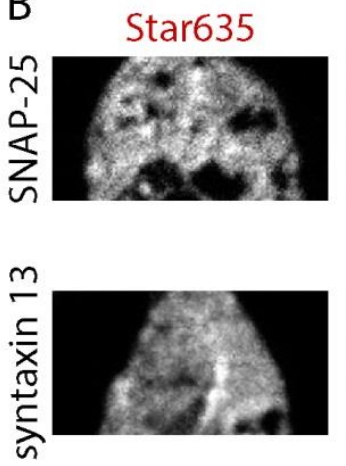

${ }^{19} \mathrm{~F}$

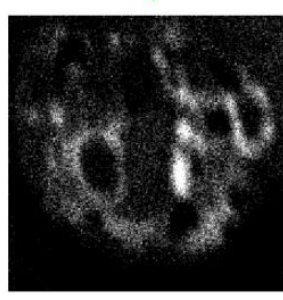

${ }^{19} \mathrm{~F}$
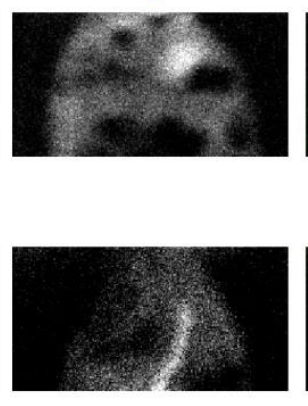

overlay

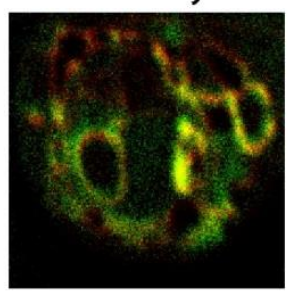

overlay
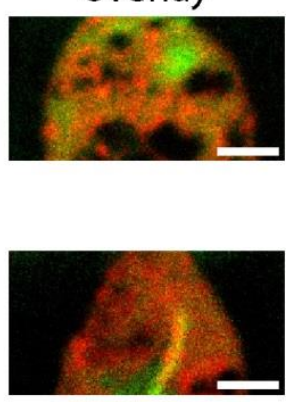

calnexin

${ }^{14} \mathrm{~N}$

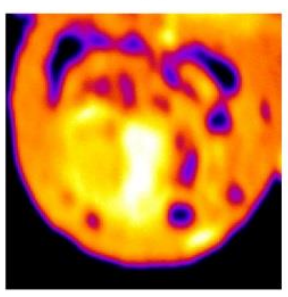

(confocal)
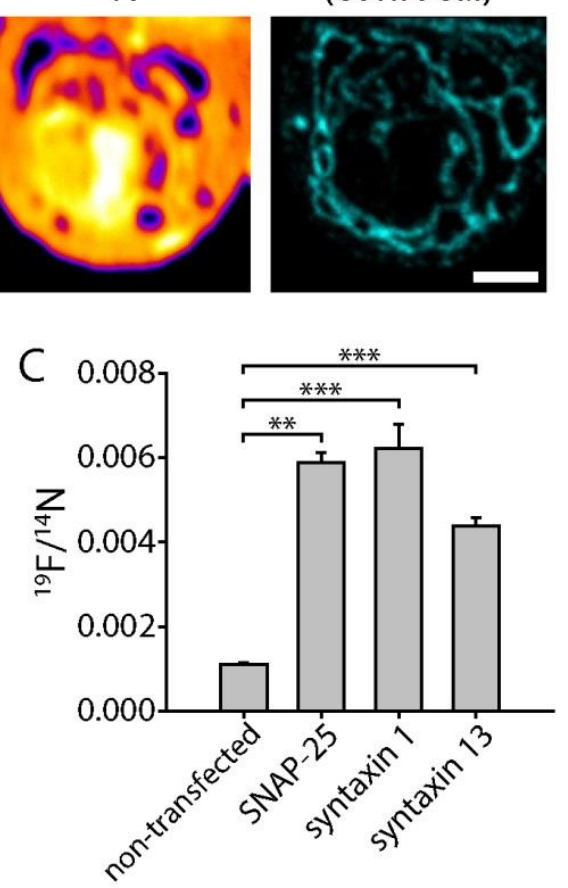

Figure 3-30 SK155 specifically labels the proteins of interest for NanoSIMS measurements

Genetic encoding of PRK into syntaxin 1 (A) or SNAP-25 and syntaxin 13 (B) and the specificity of the NanoSIMS labeling $(\mathbf{C})$. Images in confocal fluorescence and NanoSIMS of representative cells expressing the proteins of interest and labeled with SK155 are shown. For syntaxin 1, additional fluorescence and NanoSIMS images are shown: ${ }^{14} \mathrm{~N}$ in NanoSIMS, and a confocal image of calnexin. C. The ${ }^{19} \mathrm{~F}$ to ${ }^{14} \mathrm{~N}$ ratio was in cells expressing the proteins of interest and in non-transfected cells. The number of analyzed cellular regions is: 32 for non-transfected, 371 for SNAP-25, 281 for syntaxin 1, and 448 for syntaxin 13 samples. Scale bars, $2 \mu \mathrm{m}$.

The resolution of the NanoSIMS measurements reached $195 \mathrm{~nm}$ (see Figure 3-31 A). The correlation of the confocal versus the NanoSIMS labeling and detection is summarized in Figure 3-32 B: the analysis of the Star635 levels versus the values of the ${ }^{19} \mathrm{~F}$ to ${ }^{14} \mathrm{~N}$ ratio indicates a linear correlation between the signal detected in NanoSIMS $\left({ }^{19} \mathrm{~F}\right)$ and the one detected in confocal (Star635). 
A

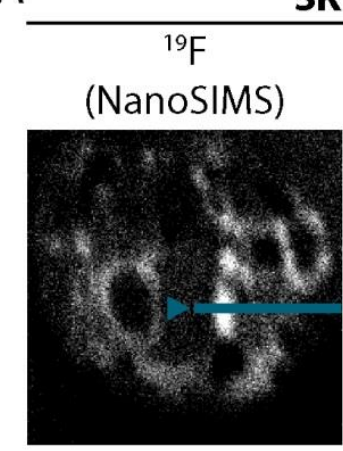

SK155
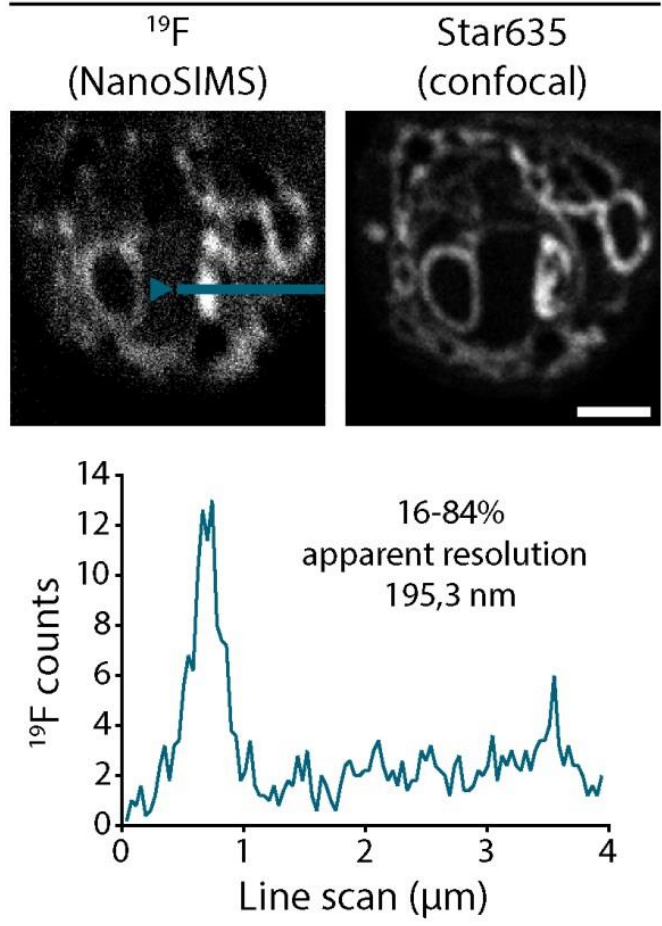

B

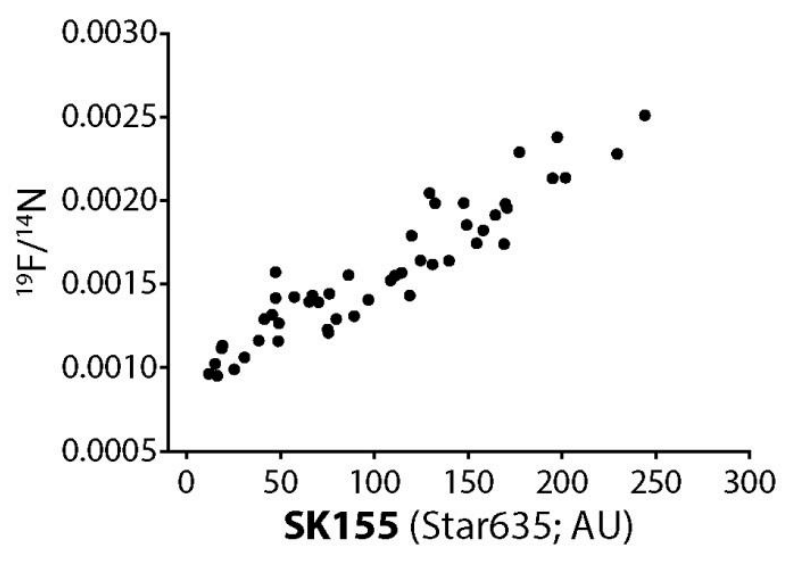

Figure 3-31 NanoSIMS resolution and correlation with confocal imaging

A. The images of a cell expressing syntaxin 1 labeled with SK155 are shown. The blue-green line shows a line scan to determine the resolution achieved in the NanoSIMS measurements. The line scan is detailed in the graph below where ${ }^{19} \mathrm{~F}$ counts are plotted and gives an apparent resolution of $195 \mathrm{~nm}$ using the $84 \%-16 \%$ rule (Lechene et al., 2006). Scale bar, $2 \mu \mathrm{m}$. B. The ${ }^{19} \mathrm{~F} /{ }^{14} \mathrm{~N}$ ratio values plotted against the SK155 (Star535) intensity levels indicate a good correlation between the confocal and the NanoSIMS signal. All values were measured in one cell. 


\subsubsection{SPILL as a Tool to Investigate Specific Protein Turnover}

Another valuable feature of SK155 is the fact that it allows for metabolic investigations with the widely used ${ }^{15} \mathrm{~N}$ isotopic marker (see Figure 3-32). In this sense, I employed this labeling tool in NanoSIMS to image at different depths the distribution of the protein of interest (in this particular example, the case of syntaxin 1 is shown) and compare it to the general protein turnover. These measurements were made possible by the very good $\mathrm{z}$ resolution of the NanoSIMS device - approximately $20 \mathrm{~nm}$ for these measurements. In this experiment, the newly produced proteins were labeled with ${ }^{15} \mathrm{~N}$-leucine and the ${ }^{15} \mathrm{~N} /{ }^{14} \mathrm{~N}$ ratio was used to identify high as well as low protein turnover cell regions. The measurements made at different depths can be pooled together to obtain summed images (summed panels in Figure 3-32). The detailed information about the distribution of the protein of interest in the context of the general protein turnover cannot be reproduced in the SK155 fluorescence image due to the much lower resolution of the confocal microscope in the $\mathrm{z}$ axis (about $700 \mathrm{~nm}$ ).

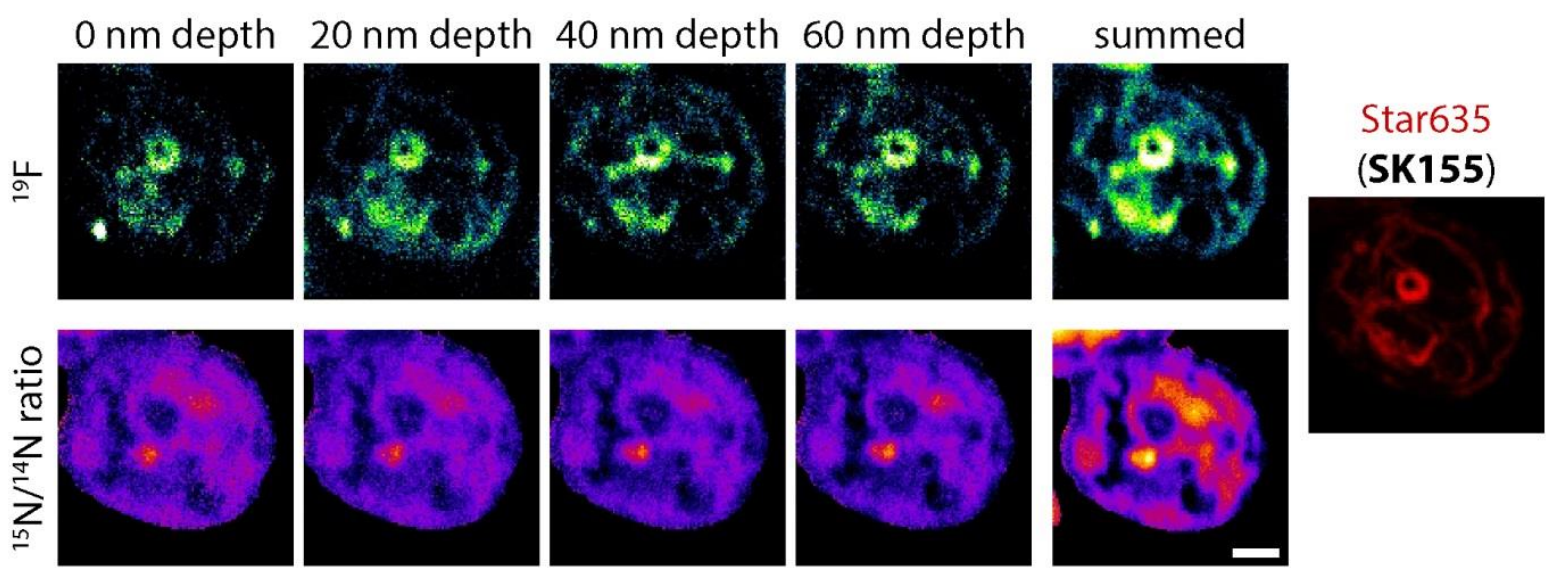

Figure 3-32 Visualizing specific protein metabolism using SPILL

NanoSIMS enables the visualization of the SK155 labeled syntaxin $1\left({ }^{19} \mathrm{~F}\right.$ images; upper row $)$ as well as the general protein turnover (given by the ${ }^{15} \mathrm{~N}$ to ${ }^{14} \mathrm{~N}$ ratios; lower rows) at different plane depths. The summed images for the ${ }^{19} \mathrm{~F}$ and the ${ }^{15} \mathrm{~N} /{ }^{14} \mathrm{~N}$ ratio are also shown. The confocal image of the same cell in the Star635 channel is given for comparison. Scale bar, $2 \mu \mathrm{m}$.

The importance of using different planes for protein turnover investigations is depicted in Figure 3-33. When looking at the ${ }^{19} \mathrm{~F}$ levels in the cell as a direct indicator of the proteins of interest (Figure 3-33 A), one can identify reliable correlations of the associations the proteins of interest form with cell regions or compartments where older or newer proteins are present. In stark contrast, if the fluorescence signal correlation with the ${ }^{15} \mathrm{~N} /{ }^{14} \mathrm{~N}$ ratio is attempted, no 
significant trend is observed (Figure 3-33 B). This is due to imprecisions in overlapping the fluorescence image with a summed stack of ${ }^{15} \mathrm{~N} /{ }^{14} \mathrm{~N}$ ratio images.

Therefore, these results mitigate the importance of using specific isotopic probes such as SK155 for metabolic studies. Compared with the fluorescence signal, the ${ }^{19} \mathrm{~F}$ offers more reliable correlation with protein turnover. As was expected, when two variables coming from the NanoSIMS are correlated (e.g. ${ }^{14} \mathrm{~N}$ and ${ }^{19} \mathrm{~F}$ ), the variance is lower than in the case when different instruments are analyzed.
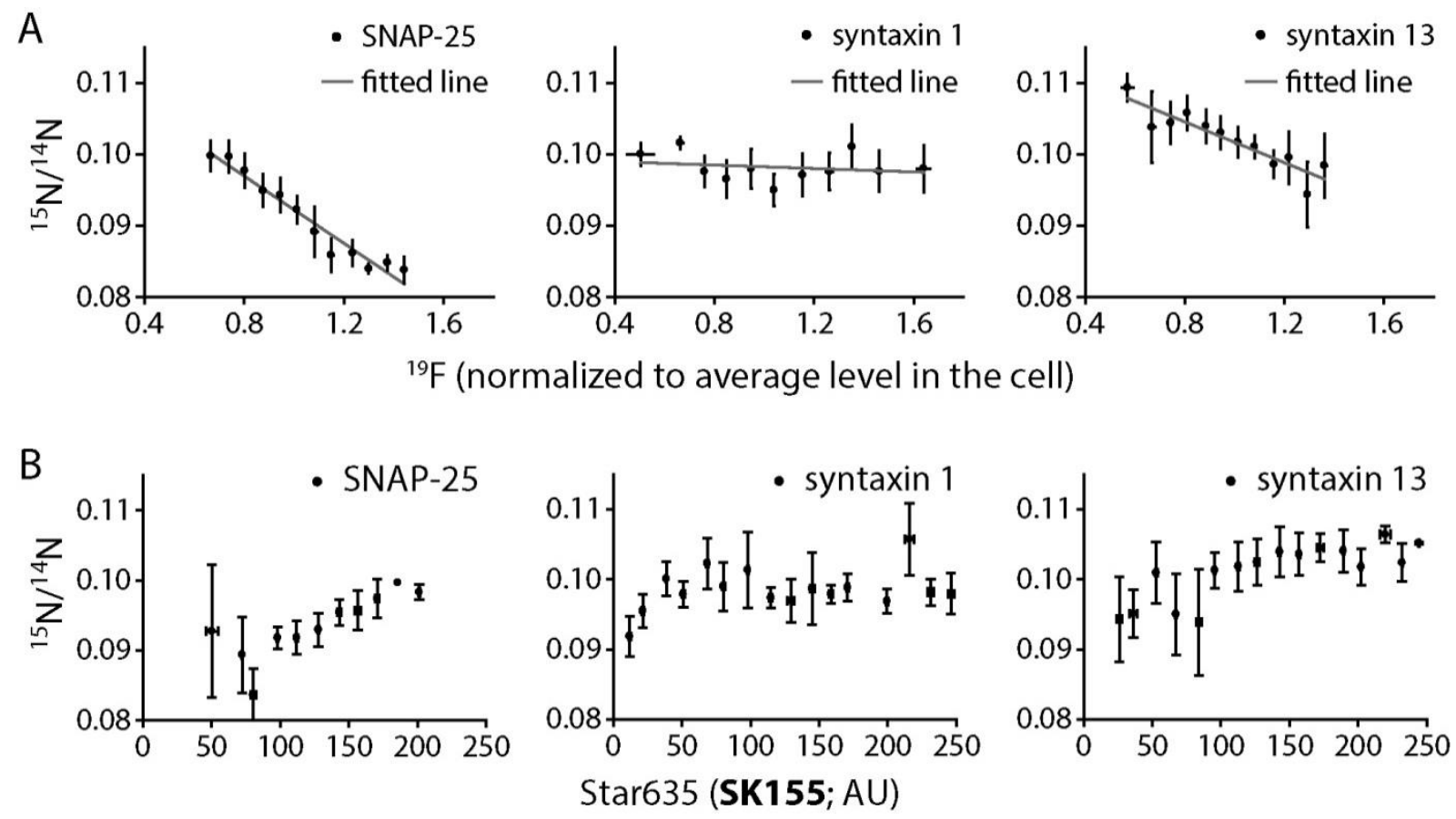

Figure 3-33 $\quad{ }^{19} \mathrm{~F}$ offers more reliable correlation with turnover than fluorescence

Analyses of ${ }^{15} \mathrm{~N} /{ }^{14} \mathrm{~N}$ ratios as a function of ${ }^{19} \mathrm{~F}$ levels (A) or Star635 fluorescence intensity (B). The number of analyzed cellular regions is 371 for SNAP-25, 281 for syntaxin 1, and 448 for syntaxin 13 samples. A. The downward trend is statistically significant only for SNAP-25 and syntaxin 13 ( $\mathrm{P}<0.01$, $\mathrm{t}$-tests), and not for syntaxin 1. B. No significant trend can be observed for the SK155 fluorescence versus turnover even though the same cell regions were analyzed. 


\section{DISCUSSION}

The main aspects that I addressed in this work involved: 1) testing if FPs are reliable labels from the point of view of specific protein organization, and 2) establishing a procedure for genetically encoding tags that are compatible with NanoSIMS imaging.

ncAA are much smaller than conventional tags such as GFP or antibodies (Figure 1-1). As a result, they can provide a more accurate picture of the structure, interactions, and turnover of proteins. In order to employ them for labeling purposes, I first tested six amino acids suitable for labeling either under copper-catalyzed conditions (pAzpa, AZK, and PRK; Figure 3-5 A) or under copper-free conditions (SCOK, BCNK, and TCNK; Figure 3-5 B).

Among the three ncAA that can undergo copper-catalyzed click reaction, PRK showed the highest specificity (Figure 3-7 and Figure 3-8). The labeling obtained with PRK also exceeded the specificity and efficiency obtained in copper-free click chemistry with the SCOK, BCNK or TCOK (Figure 3-11). As PRK offered the best trade-off between incorporation efficiency, quantitative labeling and specificity, I chose this ncAA for all further investigations into protein organization and turnover.

I then optimized the click reaction conditions for PRK, especially the type of fluorescent dye used and its concentration (Figure 3-13). When I used an oxygen scavenging system to reduce ROS species generated within the copper-click reaction mix, I did not see any significant improvement in structural integrity or GFP fluorescence intensity (Figure 3-14). So I did not include it in the final labeling protocol. I also tested the incorporation of PRK into the 26 proteins of interest investigated in this study, with good results (Figure 3-15).

PRK introduces only a minor modification in the secondary and tertiary structure of the labeled proteins. I used it, therefore, as a reporter of the possible changes in assembly/cluster organization induced by adding a FP tag to a protein. I relied for this on the incorporation of PRK into the proteins of interest either with an FP tag or without one, followed by click labeling of fixed cells and super-resolution imaging (see Figure 3-16 for a scheme of the labeling paradigm). The proteins investigated using STED microscopy (SNAP-25, syntaxin 1 and $\alpha$-synuclein; Figure 3-18) as well as the majority of the proteins investigated using GSDIM (Figure 3-19) did not show any significant differences between their non- 
tagged and their chimeric versions. The 7 proteins that showed significant differences in the detected spot size, peak intensity, and/or total intensity will be further discussed in Section 4.1.2. Using the above-mentioned parameters, I grouped the 26 proteins investigated herein according to their behavior in FP tagging (Figure 3-22). The results of this analysis indicate that, except two outliers and a small heterogenous group, the main exo- and endocytotic SNAREs grouped together and so did most of the cytosolic proteins.

For NanoSIMS investigations, I developed a technique termed SPILL (specific protein isotopic and fluorescence labeling). In this case, I applied the incorporation of PRK but carried out the click labeling reaction with isotopic probes TriazNF1 and SK155 (Figure 3-23 and Figure 3-25). These probes contain a fluorescent dye moiety (Star635), so I tested and optimized their specificity in the click reaction using fluorescence microscopy (Figure 3-24 and Figure 3-26). In addition, I analyzed the correlation of the signal detected in fluorescence with the NanoSIMS one (Figure 3-28, Figure 3-30, Figure 3-31, and Figure 3-33). TriazNF1 provided not only a background insensitive, but also a quantitative insight into the protein distribution within the cells (Figure 3-29), while SK155 enabled the visualization of the protein of interest in the context of general protein turnover (Figure 3-32).

\subsection{The Effect of FPs on the Protein Organization}

The ideal labeling tool for super-resolution imaging should be specific, induce small modifications to the target protein, and offer flexibility in the choice of fluorescent dye. Another important criterion would be the detection and/or labeling of the protein of interest under conditions (i.e. $\mathrm{pH}$, temperature, protein amounts) that are as close as possible those present in the cell.

Antibodies have been the first tools for specifically detecting proteins and their use as fluorophore conjugates has revolutionized cell biology and microscopy. However, with the advance of super-resolution microscopy techniques, their size $(\sim 13 \mathrm{~nm}$; see Table A-1) has been shown to hinder the accurate detection of their targets compared with smaller probes, such as aptamers (Opazo et al., 2012). Their bivalent nature (i.e. two paratopes per antibody molecule) may also lead to clustering of the target proteins. As antibodies are large probes, they might not penetrate well enough the samples and, especially after the embedding in plastic resin and strong fixation, the epitopes could lose their antigenicity. What is more, 
their large size and their complex structure precludes their use in live cell labeling techniques, with the exception of antibody uptake via endocytosis or surface labeling.

Smaller affinity based probes such nanobodies and aptamers present their own advantages and disadvantages. Nanobodies are small enough to be expressed both in bacteria and in mammalian cells, but they require immunization of alpacas and selection of the best binders. On the other hand, aptamers can be selected relatively fast in vitro against any target protein. However, they require solid phase synthesis and cell permeabilization to reach intracellular targets. In most of the cases, both aptamers and nanobodies selected to recognize a protein under native conditions show reduced affinity for their targets upon fixation (and viceversa).

\subsubsection{Implications on Studies Involving Fluorescent Proteins}

For live protein labeling purposes, the probe should either be genetically encoded and/or cross the cell membrane without permeabilization.

GFP has been the first genetically encoded fluorescent tag. To this day, it is still among the most widely used in cell biology for visualizing proteins in both live and fixed cells. For instance, all of the 26 proteins included in this study have been investigated as FP chimeras. In view of the many studies involving FP tagged proteins, it would be advantageous to assess to which extent this tag affects protein organization and obtain a validation of studies using FP tags.

The main reason behind the widespread use of FPs is their convenience: the protein of interest needs only to be expressed with an N-terminal or C-terminal tag. Stably folded Nterminal tags are not indicated for secreted or mitochondrial proteins because the tag can affect their translocation and lead to the mislocalization of the protein (Denzer et al., 1995; Gonzalez et al., 2009). C-terminal tags are a better option in such cases, because the protein of interest retains its proper localization (Palmer and Freeman, 2004) and the FP shows better fluorescence properties (Tajima et al., 2010). Yet, even C-terminal FP tags may cause problems in protein folding and post-translational translocation to the ER for tail-anchored proteins. The latter type of proteins are defined by the presence of a single C-terminal transmembrane domain (Kutay et al, 1993). In such a case, the C-terminal FP tag can change the mechanism for insertion in the ER membrane from post-translational to cotranslational. In other cases, the mislocalization can be due to the expression levels of the protein of interest. If the protein is too strongly overexpressed, especially in the presence of its 
endogenous form, this can result in the spurious intracellular localization (Opazo et al., 2010; Stadler et al., 2013). Other problems that arise due to FP tagging address the organ and/or organismal level. For instance, $\beta$-actin-GFP mice are affected by kidney defects (glomerulosclerosis) (Guo et al., 2007).

FPs have been shown to induce clustering artifacts of tagged proteins when imaged using PALM (Annibale et al., 2011a). These artifacts could originate in the intrinsic affinity that FPs have for one another, especially to form dimers (Jain et al., 2001; Phillips, 2001; Chen et al., 2002). This intrinsic affinity might lead to the formation of protein dimers and oligomers in cases where such interactions do not physiologically occur. Another effect is the formation of larger protein assemblies for the tagged proteins than would be the norm. There have been efforts to reduce the homo-binding of GFP binding through directed mutagenesis approach (von Stetten et al., 2012). However, in this study the proteins were tagged with the enhanced GFP (EGFP, referred throughout the manuscript as simply GFP) reported to form dimers (Jain et al., 2001; Chen et al., 2002).

If the relatively large size of an FP tag is taken into account $(5.1 \times 4.2 \times 3.22 \mathrm{~nm} ; 26 \mathrm{kDa})$, the effect it has on the protein of interest should be the opposite. More explicitly, the FP tag should affect the ability of proteins to associate into clusters or correctly bind to interaction partners.

Peptide tags, such as the SNAP, Halo or the tetracysteine tag, are smaller alternatives to GFP. Yet even they may affect the secondary and tertiary structure of the proteins they label. For example, the tetracysteine tag, which is the smallest among these tags, has been shown to alter the Gag protein localization depending on the position where it is inserted in the protein (Rudner et al., 2005).

This is why I turned to the smallest possible tags for proteins - the non-canonical amino acids. ncAAs offer flexibility with regard to the position in the protein of interest where they can be integrated and impart the least possible disruptions and impairments in protein structure and function. In addition, they offer versatility in terms of the fluorescent probes that can be later coupled to them. Therefore, a major part of my project was to use ncAAs as tools to investigate the possible effects of FP tagging on the organization of a cohort of 26 proteins. All protein-FP constructs used in this study were C-terminal tagged and showed proper cellular distribution (Figure 3-15).

To answer the question if FP tags affect protein organization, I used ncAA for protein labeling. My approach was to incorporate the non-canonical amino acid PRK into 
exogenously expressed FP tagged and non-tagged proteins and label them with dyes for super-resolution microscopy. STED microscopy investigations at a $30 \mathrm{~nm}$ resolution revealed no significant difference between the tagged and non-tagged proteins SNAP-25, syntaxin 1 and $\alpha$-synuclein (Figure 3-18). For a more comprehensive analysis of the effects of FP-tagging, I turned to the pointillistic super-resolution method GSDIM (Figure 3-19). Out of the 26 proteins, only PIPKI $\gamma$ and VAMP4 exhibited significant differences in all the investigated parameters: spot size, peak intensity and summed intensity of the spot (Figure 3-20). The other five proteins showed significant results either in the sizes of the detected spots (Vti1a- $\beta$ ), total spot intensity (syntaxin 6 ) or both peak intensity and total intensity ( $\beta$ actin, amphiphysin, and Munc18-1). The particular proteins cases for which significant differences between their tagged and non-tagged forms were found will be discussed in Section 4.1.2. Therefore, the assemblies formed by FP-fusion proteins shows little alterations in size compared to their non-tagged counterparts. The spot intensity parameters indicate that the composition and density of the protein assemblies is affected to a somewhat larger extent.

\subsubsection{Proteins Affected by FP Tagging}

In this project I analyzed the organization of both cytosolic and membrane attached proteins. Among the cytosolic proteins I investigated, $\beta$-actin, amphiphysin, Munc18-1, and PIPKI $\gamma$ showed significant difference when FP tagged (Figure 4-1). Only three membrane attached proteins were affected in different extent when fused to FPs. 


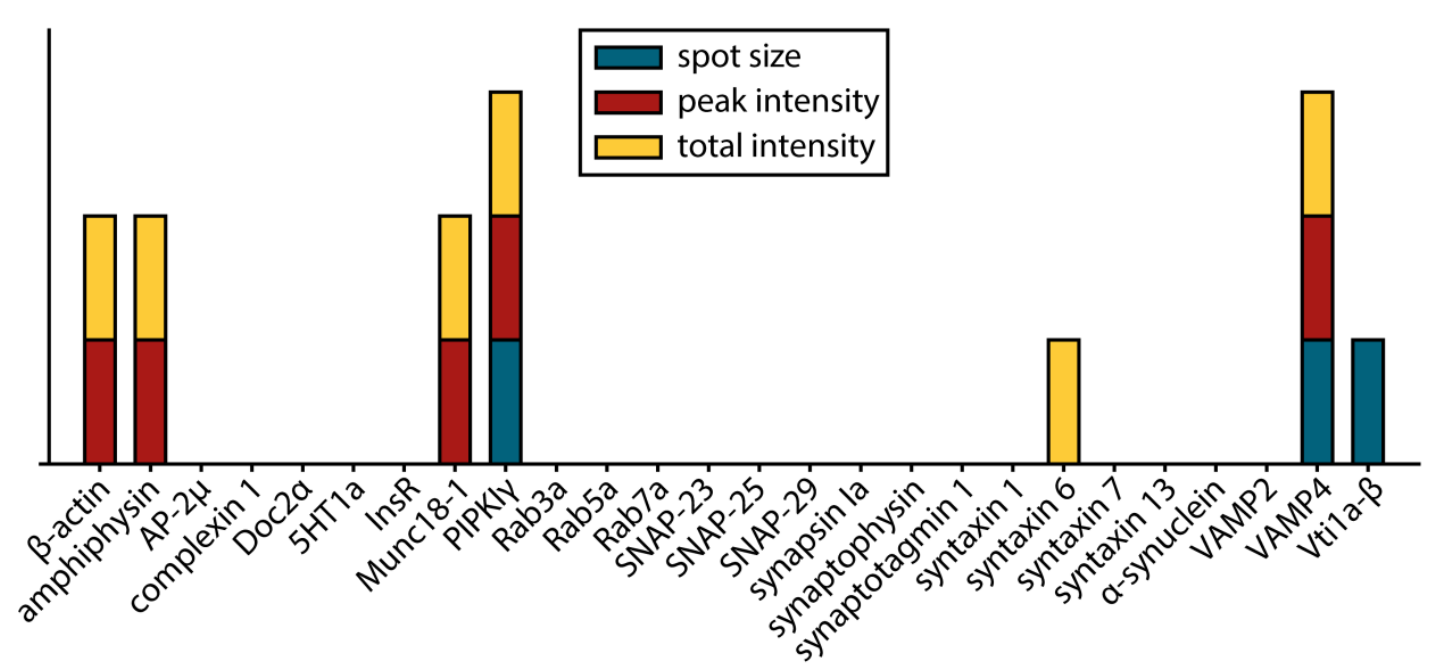

\section{Figure 4-1 Overview of significance in t-tests for the investigated proteins}

Graph depicting the results in Student's t-test for the three parameters that were assessed. If the difference between the FP-tagged and the non-tagged version of the protein of interest is statistically significant a rectangle filled in with blue-green (for spot size), in red (for peak intensity), or in yellow (for the total intensity) is shown above the protein name.

\section{Cytosolic Proteins}

PIPKI $\gamma$ (phosphatidylinositol 4-phosphate 5-kinase type-1 gamma) concentrates at synapses where it acts as the major generator of PtdIns(4,5)P2 (Ishihara et al., 1998; Wenk et al., 2001) and controls major processes, such as actin remodeling, cell adhesion, and endocytotic vesicle transport (Di Paolo et al., 2002; Doughman et al., 2003; Krauss et al., 2006; Li et al., 2013). Pietro De Camilli and colleagues proved the 28-aa C-terminal tail in PIPKI $\gamma$ is essential for its association with focal adhesions (Di Paolo et al., 2002). PIPKI $\gamma$ showed a highly significant change in all three parameters investigated.

The detected spots of FP tagged PIPKI $\gamma$ are $~ 60 \%$ bigger and both the peak intensity and the total intensity of the spots are $\sim 3$-fold higher. The isoforms $\alpha$ and $\beta$ of PIPK have been recently shown to dimerize with important consequences on their enzymatic activity and interaction with other proteins and membranes (Hu et al., 2015; Lacalle et al., 2015). For PIPKI $\gamma$, such studies are missing but the results herein suggest that FP tagging induces the formation of higher order oligomers. Even though PIPKI $\gamma$ has been used so far only as Nterminal GFP fusions (Di Paolo et al., 2002), the C-terminal chimera used in this study has shown proper localization. Interestingly, the PIPKI $\gamma$ interaction partner AP- $2 \mu$, which binds and clusters around the endocytotic cargo (Krauss et al., 2006; van den Bout and Divecha, 
2009), did not show any significant differences in the protein assemblies it forms as an FP chimera.

Cytosolic proteins $\beta$-actin and Munc18-1 have spots brighter by $~ 50-60 \%$ upon FP tagging, both for peak intensity and total peak intensity. Yet, their spot size does not differ significantly. This finding suggests that FP fusions associate in higher numbers into multimers that retain more-or-less similar sizes like their non-tagged variants.

$\beta$-actin has been widely used for in vivo and in vitro experiments as an C-terminal chimera with FP (Hodgson et al., 2000; Guo et al., 2007; Hamamoto et al., 2010). This protein is a major component of the cytoskeleton, forming microfilaments by binding to four other actin molecules (Holmes et al., 1990; Chen et al., 2000). So, for $\beta$-actin, it is easy to imagine that the FP tag can modify the geometry of $\beta$-actin assembly into oligomers, leading to the observed differences. The molecular arrangements formed by actin were shown to be very sensitive to sample preparation. For example, the periodical cytoskeleton formed by actin along the axon could be observed either using in vivo labeling techniques in STED (D'Este et al., 2015) or very careful embedding procedures in STORM (Xu et al., 2013).

Munc18-1, also known as syntaxin-binding protein 1, is a neuronal protein involved in synaptic transmission, especially vesicle docking and priming (Verhage et al., 2000; Deák et al., 2009). It has also been shown to bind to syntaxin 1 with high affinity and mediate its transport to the cell membrane. In addition, Munc18-1 regulates the activity of syntaxin 1 (Toonen et al., 2005; Gerber et al., 2008) and also binds the exocytotic SNARE complex (Deák et al., 2009; Meijer et al., 2012). For the Munc18-2 isoform, a point mutation has been shown to induce dimerization without affecting the ability of this protein to bind its interaction partner syntaxin 11 (Hackmann et al., 2013). No evidence for Munc18-1 dimerization or multimerization exists. However, the $\sim 50 \%$ increase in spot brightness detected here indicates that the FP tag may increase the self-association of Munc18-1.

Amphiphysin is a protein which associates with synaptic vesicles and presynaptic membranes (Lichte et al., 1992). It contains an N-terminal BAR (Bin-Amphiphysin-Rvs) domain through which it interacts with membranes and induces their tubulation (Takei et al., 1999). The BAR domain can induce the dimerization of amphiphysin (Peter et al., 2004). The amphiphysin isoform 1 (brain-specific) and isoform 2 (widely distributed) are also known to form heterodimers (Wigge et al., 1997) and homodimers mediated by the BAR domain (Peter et al., 2004). Amphiphysin 1 (mentioned throughout this work as amphiphysin) formed spots dimmer by $\sim 20 \%$ when FP tagged, both in terms of peak 
intensity and total intensity. As the spot size is not affected, one possible explanation would be the association of fewer amphiphysin molecules in the presence of an FP tag, while retaining the overall size of the assembly remain unchanged. One plausible cause for this observation is the steric hindrance imposed by the FP tag.

\section{Membrane Proteins}

Among the membrane-attached or integral proteins, VAMP4 exhibits the biggest differences: its FP chimera forms assemblies smaller by more than $20 \%$ and dimmer by $\sim 50 \%$. A similar construct to the one used in this project, VAMP4-GFP, was shown to cycle between the cell membrane, recycling endosomes, and trans-Golgi network due to an $\mathrm{N}$ terminal motif (Zeng et al., 2003; Tran et al., 2007). It is currently not known if VAMP4 associates into clusters like other SNARE proteins have been shown to do (Sieber et al., 2007; Halemani et al., 2010; Bar-On et al., 2012). But these results indicate that the presence of an FP tag inhibits VAMP4 association. In constrast to constitutively expressed VAMP4, the neuronal VAMP2 did not show any significant differences in any of the investigated parameters.

Another SNARE protein, Vti1a- $\beta$, forms somewhat bigger assemblies (by $\sim 20 \%$ ) when FPtagged, without modifications in spot brightness. This points to a possible expansion of the protein assembly to accommodate the FP tag, without modifying the number of protein molecules per cluster. Vtila- $\beta$ is a brain-specific splice variant enriched in small synaptic vesicles (Antonin et al., 2000). Vesicles containing Vtila participate in spontaneous fusion (Ramirez et al., 2012). However, no data is available with regard to its clustering behavior.

Interestingly, among the four types of syntaxin included in this study, only one of them, syntaxin 6 , showed a significant difference in the total intensities of the detected spots but not of the spot size or the peak intensity. This could be the result of very small modifications in the organization of the assemblies formed by syntaxin 6 . Such small intensity differences can be detected only using the total intensity analysis, which is more sensitive to dimmer spots. It is worth to note that syntaxin 7 also showed big differences in the parameter values when FP tagged, but these were not significant due to the large standard error of the mean. Interestingly, syntaxin 13 (also known as syntaxin 12), a binding partner of syntaxin 6, is not affected by FP tagging. Syntaxin 1, a neuronal SNARE protein found on the plasma membrane, did not show differences in the clusters it formed in the presence or absence of a C-terminal YFP tag neither in STED nor in GSDIM. Possible reasons why only syntaxin 6 is more susceptible to FP tagging than the other syntaxins are the sizes of the transmembrane 
domains and the vesicular/extracellular exposed tails. According to Uniprot (www.uniprot.org), syntaxins 6, 7 and 13 all have transmembrane domains with 21 residues, while syntaxin 1 has 23 . If the residues of the cytoplasmic tail are taken into consideration, then syntaxin 6 has no exposed residues in its tail, whereas syntaxin 7 and syntaxin 13 have 2 and 3, respectively. Hence, tagging with FP may induce stronger perturbations in syntaxin 6.

In principle, larger protein assemblies should exhibit also a higher total intensity, and viceversa. This is indeed the case for PIPKI $\gamma$ and VAMP4, the two proteins that showed the most pronounced differences (Figure 3-20). For the rest of the proteins, only small but significant differences in either the size or the intensity were observed. A possible explanation for this is that fluorescent dye molecules can undergo self-quenching in tightly packed protein clusters (Saka et al., 2014a). This observation is in line with the case of Vtila- $\beta$, which forms bigger protein assemblies in the presence of an FP tag, but no increase in total fluorescence intensity is detected. In contrast, loose protein assemblies would describe protein clusters that are dim but not very dense. So they can accommodate more proteins or lose them without changing their size. If all other technical and conceptual biases are removed, this would be the case for the rest of the proteins that show significant differences only in their spot intensity profile ( $\beta$-actin, amphiphysin, Munc18-1, and syntaxin 6) 


\subsection{Implications for NanoSIMS Investigations}

\subsubsection{Specific Labels for NanoSIMS}

Detection of a protein of interest in NanoSIMS requires isotopes or elements that are not naturally found in biological samples or that are found only in trace to small amounts. General approaches to labeling proteins have involved the use of canonical amino acids containing stable heavy isotopes, such as of ${ }^{13} \mathrm{C}$ or ${ }^{15} \mathrm{~N}$ (Lechene et al., 2006). However, such methods do not allow the visualization of one specific protein in NanoSIMS and require correlation with immunofluorescence (Saka et al., 2014b).

Another approach is described by Nolan and collaborators and involves the use of antibodies conjugated with lanthanides to identify up to ten different proteins directly in NanoSIMS (Angelo et al., 2014). As discussed above, the large size of the antibodies deters their access and binding to many epitopes. What is more, this technique requires coupling of the antibodies with metals in a chemical reaction that could damage the immunoreactivity of the antibody. These can lead to low sensitivity of the assay and even false negative results.

The goal of this study was to design and characterize smaller and more sensitive probes for detecting proteins in NanoSIMS. Another desirable feature was to render the correlation with fluorescence microscopy optional. As previously discussed, ncAAs, and in particular PRK, fulfill all these criteria if they are coupled to appropriate isotopic probes, in the technique termed SPILL. The probes I have used in this study rely either on ${ }^{15} \mathrm{~N}$ (TriazNF1) or on ${ }^{19} \mathrm{~F}$ (SK155) for their signal (Figure 3-28 and Figure 3-30). SPILL achieves a better resolution and a higher sensitivity compared with the method proposed by Angelo et al., 2014. Moreover, the TriazNF1 probe enables background-insensitive measurements of the copy numbers for the protein of interest.

SK155 offers an alternative approach to a high signal-to-noise ratio based on the minimal concentrations of ${ }^{19} \mathrm{~F}$-containing compounds in soft tissues (Longmaid et al., 1985; Daugherty et al., 1989). In addition, this probe enables concomitant protein turnover investigations using ${ }^{15} \mathrm{~N}$-leucine as a general protein marker. The applications of this probe for protein turnover studies will be further discussed in Section 4.2.2. 


\subsubsection{Specific Protein Turnover Revealed by SPILL}

Correlating NanoSIMS with super-resolution microscopy (Saka et al., 2014b) provides information on protein turnover occurring in different organelles. But the use of large antibodies and the difficulty to match the NanoSIMS and confocal measurements, make such studies laborious and may lead to large experimental errors that mask significant information.

The two most widely employed isotopes for marking newly synthesized proteins are ${ }^{15} \mathrm{~N}$ and ${ }^{13} \mathrm{C}$ (Lechene et al., 2006; Steinhauser and Lechene, 2013). The general labeling for protein metabolism offered by the ${ }^{13} \mathrm{C}$ to ${ }^{12} \mathrm{C}$ ratio has an unsatisfactory signal-to-noise ratio (Figure 3-28 B). This issue forestalled the protein turnover investigations for the TriazNF1 probe.

On the other hand, SK155 is compatible with protein turnover studies using the ${ }^{15} \mathrm{~N}$ isotope. The correlation between the ${ }^{19} \mathrm{~F}$ signal and the fluorescence signal is indisputable when the summed NanoSIMS images are taken into account (Figure 3-30 and Figure 3-31), both techniques reaching a similar lateral resolution of $\sim 200 \mathrm{~nm}$. However, there are stark differences in the axial resolution: the NanoSIMS measurement included in this study reached a $20 \mathrm{~nm}$ resolution, while the resolution of the confocal was dictated by the thickness of the embedded section (200 nm). Most importantly, as shown in Figure 3-33, the correlation between the fluorescence and the NanoSIMS signal could fail to reveal significant information due to the vastly different imaging conditions.

The correlation between protein turnover, indicated by the ${ }^{15} \mathrm{~N}$ to ${ }^{14} \mathrm{~N}$ ratio, and the ${ }^{19} \mathrm{~F}$ signal provided a much more reliable correlation. Interestingly, SNAP-25 and syntaxin 13 exhibited a significant tendency to associate with cell regions and/or compartments where proteins contain less ${ }^{15} \mathrm{~N}$. This was not the case for syntaxin 1 . Little information about the turnover of these SNARE proteins is found in literature, hinting that such studies would be advantageous for this field. Syntaxin 1 and SNAP-25 were found to have faster turnover in developing neurons than in mature ones, with SNAP-25 showing a marked increase in halflife from 16 hours (5 DIV) to 35 hours (14 DIV). For syntaxin 1 the differences were smaller and oscillated around 48 hours (Sanders et al., 1998). Considering the half-life for SNAP-25 in BHK cells is more or less equal to the one in mature neurons (35 hours), then most of the SNAP-25 that has incorporated PRK during the 18-hour expression did not start to be degraded. By the end of the 3-day incubation with ${ }^{15} \mathrm{~N}$-leucine, most of the general proteins with fast and medium turnover are labeled with ${ }^{15} \mathrm{~N}$. With the current data, it is difficult to 


\section{Discussion}

infer whether SNAP-25 (and syntaxin 13) have a preference for slow turnover proteins (> 3 days) or for the very fast ones ( $<2$ hours).

\subsection{The Experimental Approach and its Limitations}

In this next section I will address technical caveats. One limitation of the ncAA incorporation technique is the ectopic expression of the proteins of interest together with the suppressor RS/tRNA pair (Liu and Schultz, 2010). For comparison purposes, all proteins investigated herein were expressed under the regulation of the human cytomegalovirus (CMV) promoter. This is a commonly used constitutive promoter and has been shown to have variable strength in driving transcription depending on the cell type in which expression takes place (Qin et al., 2010). In BHK cells, the CMV promoter has been shown to be the strongest promoter to drive ectopic expression (Yano et al., 1994).

\subsubsection{Non-Canonical Amino Acid Incorporation Technique}

Molecular biology and genetic engineering are limited, with few exceptions discussed below, by the 20 amino acids present in the canonical genetic code. The latter makes use of all the 64 possible triplet combinations. So the endeavor to expand the repertoire and chemical properties of proteins using ncAAs had to rely on the least used codon. The best candidate for this was the Amber stop codon (Liu and Schultz, 2010). Ochre and Opal stop codon suppression (Köhrer et al., 2004) as well as quadruplet decoding (Anderson et al., 2004) have also been described, but have lower efficiencies. In practice, the Amber stop codon is for now the most widely used for ncAA incorporation. This restricts the number of proteins that can be labeled to only one.

The ultimate goal of genetic code expansion approach is to evolve a complete bioorthogonal machinery starting from an unassigned codon and continuing all the way to a modified ribosome that efficiently decodes the unassigned codon (Anderson et al., 2004; Neumann et al., 2010). Such an approach would open the possibility for multiprotein labeling in one cell. The efficiency of ncAA incorporation ranges between 20\% (Blight et al., 2004; Wang et al., 2007). This is due to the competition with the endogenous release factors that also recognize the Amber codon and results in the production of truncated protein variants. The incorporation efficiency is influenced by the position of the Amber codon in the coding 
sequence of the protein of interest and by the amounts of suppressor RS/tRNA present in the cell (Pott et al., 2014). Improvement in ncAAs incorporation has been achieved by increasing the amount of orthogonal tRNA expressed in the cell and by adapting the promoter and terminator to the host cell. This leads to a higher amount of suppressor tRNA in the expressing cell and as a result, the tRNA can outcompete the release factor 1 , thereby improving ncAA incorporation from approximately $\sim 10 \%$ to more than $90 \%$ as proven in a $\beta$-galactosidase assay in bacteria (Ryu and Schultz, 2006). For mammalian cells, the plasmids contain a tandem repeat of multiple orthogonal tRNA genes (from 3 up to 9 copies) and the tRNA transcription was enhanced using human tyrosyl-tRNA 5' flanking sequences, thus more than doubling the amount of heterologous tRNA (Sakamoto et al., 2002; Liu et al., 2007).

Recently, Isaacs and collaborators have engineered an E. coli strain which has all of its Amber stop codons mutated to Ochre ones (Lajoie et al., 2013). This genomically recoded organism (GRO) is also lacking the release factor 1 (RF1), which is responsible for polypeptide chain release in prokaryotes in response to Amber and Ochre stop codons. This converts the Amber codon into a blanck one that can be then reassigned to incorporate any ncAA using an orthogonal RS/tRNA pair. Such an organism would of course express proteins containing ncAAs with a very good yield and high purity. However, such an approach for eukaryotic organisms, even simple ones, is not practical. This is because, compared to the 7\% occurrence of Amber codons in prokaryotes, the eukaryotic genome has a preponderance of approximately $23 \%$ with respect to all stop codons (Liu et al., 2007). In addition, there are many more genes for the eukaryotes. Another issue would be the fact that eukaryotes have only one release factor which recognizes all stop codons (Kisselev et al., 2003).

In spite of the large amount (23\%) of proteins in mammalian cells that have Amber as stop codons, Chin and colleagues demonstrated no-significant amounts of spurious proteome labeling (Uttamapinant et al., 2015). In their setup the Amber mutants are expressed at levels comparable or even lower than for the rest of the proteins present in the cell. The suggested reasons include on one hand the context the Amber stop codon is placed under (i.e. the natural termination site versus an artificial one which lacks proper signaling elements) and, on the other hand, the degradation of the abberant proteins (i.e. longer than normal) resulting from the suppression of the natural Amber stop codon. 
At the moment, the number of ncAAs available for incorporation greatly exceeds the codons suitable for suppression. Aside from the clickable ncAA, which will be described below, the incorporation of fluorescent acids has been described (Lepthien et al., 2008; Merkel et al., 2010). But these tryptophan-derived amino acids have poor photophysical properties and are not amenable to super-resolution microscopy. What is more, they lack the flexibility the clickable ncAA offer with regard to the choice of the fluorophore that can be coupled.

\subsubsection{Click Reaction}

The optimal click reaction between a ncAA and a fluorescent probe should be not only specific and quantitative, but the final labeling result should also provide a high signal-tonoise ratio (contrast).

On the fixed samples (membrane sheets or cells) used in this study, the copper-catalyzed click reaction proved to be the most reliable (Section 3.2). However, CuAAC presents a series of drawbacks when applied on live cells, the most serious issue being the cytotoxicity of the copper ion (Link and Tirrell, 2003). Heavy metal ions such as $\mathrm{Cu}^{2+}, \mathrm{Pb}^{2+}, \mathrm{Hg}^{2+}$ or $\mathrm{Al}^{3+}$ have been shown to crosslink proteins and DNA (Wedrychowski et al., 1986). The required concentrations to observe an effect using high speed centrifugation of protein-DNA complexes are: $0.5 \mathrm{mM}$ for $\mathrm{CuSO}_{4}, \mathrm{HgCl}_{2}$, and $\mathrm{AlCl}_{3}$, while a higher concentration amounting to $5 \mathrm{mM}$ is necessary for $\mathrm{Pb}\left(\mathrm{NO}_{3}\right)_{2}$. In the experiments included in this work, the final concentration of $\mathrm{Cu}^{2+}$ in the click reaction mix applied to the cells was $5 \mathrm{mM}$. A lower concentration (e.g. $1 \mathrm{mM}$ ) significantly impairs the labeling reaction. As the proteins are fixed prior to the application of the click reaction mixture, I do not expect major structural changes induced by the $\mathrm{Cu}^{2+}$ ions.

The click reaction in the presence of copper also generates reactive oxygen species which generate radical-induced damage to the both the proteins of interest and the fluorophores present in the sample. To counteract this putative process, I used ROS scavenger system recently described and named ClickOx by Markus Sauer and colleagues (Löschberger et al., 2014). The ClickOx reaction mixture does not provide any protection to the expressed GFP fluorophores compared with the normal click mix (Figure 3-14). Therefore, under the conditions that I employed for the experiments, namely 30 minutes incubation time and $5 \mathrm{mM}$ concentration of $\mathrm{Cu}^{2+}$, the ClickOx system does not bring any improvement in terms of better signal for the GFP moiety or for the synthetic dye. 
For copper-free click chemistry, the SPAAC takes too long to be effective under live labeling conditions. The much faster SPIEDAC reaction is therefore preferable. Both methyl-tetrazines and simple non-substituted ones have been reported to react with strained alkyne or alkene groups (Nikić et al., 2014; Uttamapinant et al., 2015). In this study I have employed the commercially available Atto647N-tetrazine and the in-house produced KK114-tetrazine. However, under physiological conditions it would be preferable to employ methyl-substituted tetrazine dyes because only these derivatives are stable enough under physiological conditions.

\section{ncAAs for CuAAC}

According to Lemke and collaborators all azide derivatives are generally sensitive to reduction and their alkyne counterparts are more indicated for labeling purposes (Milles et al., 2012). But aromatic azides are even more liable and generally tend to be reduced by thiol groups (Staros et al., 1978). This makes all azides not as well suitable for labeling cells in culture as amino acid derivatives containing alkyne groups. The main problem would be that the amino acid is incorporated but, due to its sensitivity to reduction, the azide group is inactivated and, therefore, becomes unable to undergo click reaction. As a result no labeling (or very little) is detected.

The same work from Lemke and collaborators confirms through single molecule FRET studies that the aliphatic ncAAs are preferable to the aromatic ones (Milles et al., 2012). This is because the latter are considerably stiffer (i.e. can adopt only a few conformation due to the aromatic ring). This may induce conformational problems in the target protein after incorporation.

In line with the results presented herein, Lemke and collaborators have also reached similar conclusions from experiments on bacteria (Milles et al., 2012). In short, PRK is better than AZK, pAzpa and its alkyne analogue, p-(propargyloxy)-L-phenylalanine, in terms of expression yield, chemical stability, linker flexibility, reaction speed in $\mathrm{CuAAC}$, and labeling efficiency under physiological conditions.

\section{ncAAs for SPAAC and SPIEDAC}

All three amino acids, SCOK, BCNK and TCOK, have been reported to undergo a click reaction in the absence of any metal catalyzer (Plass et al., 2011, 2012; Nikić et al., 2014; Uttamapinant et al., 2015). 
My initial tests using flurophores with azide groups led to unsatisfactory results. So I resorted to tetrazine derivatives of dyes less hydrophobic than Atto647N.

All the copper-free ncAAs tested in this project: SCOK, BCNK and TCOK, have been used in a double labeling procedure (Nikić et al., 2014). Chin and collaborators have also successfully employed BCNK and TCNK for the labeling of surface proteins in superresolution microscopy investigations (Uttamapinant et al., 2015). However, all these labeling reactions were performed on surface proteins expressed by live cells.

Many of the proteins included in this study are either cytosolic or are membrane attached and do not protrude on the surface of the cell. Therefore, I tried to first identify whether these ncAAs also perform well in copper-free click chemistry on fixed and permeabilized samples. To begin with, I realized early that SPAAC, which involves the reaction between a strained amino acid and an azide derivative is not efficient enough to provide proper labeling (Figure 3-9). Interestingly, BCNK has been reported to be more suitable than TCOK for ncAA incorporation and SPIEDAC labeling (Uttamapinant et al., 2015). In the experiments from Figure 3-11, I have compared the three available copper-free ncAAs SCOK, BCNK and TCOK and found that BCNK does not show any significant labeling in BHK cells, while the labeling provided by SCOK is insufficient (low levels). TCOK showed the strongest signal, in accordance with its high reactivity in SPIEDAC (Uttamapinant et al., 2015). However there was a strong background in all the cells treated with this ncAA. This is most likely caused by the difficulty to remove during the 2-hour wash before fixation the unincorporated TCOK from the cells (Uttamapinant et al., 2015).

The discrepancy between the previously reported findings for $\mathrm{BCNK}$ and the results presented herein could be related to the fact that I used a racemic mixture of exo and endo enantiomers (ratio endo:exo=1:2), while the other research groups might have used racemic mixture enriched in the more reactive isomer (the exo-isomer was four-fold more abundant than the endo one in the report of Uttamapinant et al., 2015). Therefore, as an outlook I would try to further optimize the BCNK click reaction by incorporating the enantiomerically pure exo BCNK isomer. Another possible reason is the fact that the BCNK stock was rather old (approximately two years) and it might have been degraded during storage at $-20^{\circ} \mathrm{C}$. 


\subsubsection{Fluorescent Dyes}

Fluorescent dyes have different hydrophobicities and this influences the background in the staining assay as well as their ability to accurately label proteins/structures of interest (Hughes et al., 2014). The moderately hydrophylic Atto647N binds nonspecifically to membranes and other hydrophobic cell components during click reactions (Figure 3-12). This is in line with the observations that Atto647N displays disadvantageous binding to glass and has a big affinity for mitochondria (Kolmakov et al., 2010). KK114, on the other hand, shows no background even at a higher concentration compared with Atto. This highly soluble dye is therefore a more reliable labeling probe. The negative net charge hinders membrane permeability in KK114 (-1) and Star635P (-3), but improves solubility. The sulfonic acid groups improve not only the solubility of the dye, but seem to also prevent aggregation and improve quantum yield compared with Atto647N (Kolmakov et al., 2012). As a result, this dye can be used at a higher concentration during cellular labeling experiments.

Boxer and collaborators have established that Star635P and, to an even lower extent, Alexa647 show only low binding coefficients to membranes (Hughes et al., 2014). Note that Star635P is the brightest (i.e. has the highest fluorescence quantum yield) among the three rhodamine dyes used in this study when conjugated with antibodies (Kolmakov et al., 2012). This is a good indicator that when this dye is covalently linked to the protein of interest, it retains a strong fluorescence that would further enable super-resolution imaging due to the good signal. So I have chosen it for the final experiments.

For NanoSIMS applications, where most of the concern was to obtaining the probes in good yield and with high purity, the more stable Star635 rhodamine dye was employed (see Figure 3-25). Nevertheless, the Star635 fluorophore has one of the highest quantum yields for near infrared dyes, second only to Star635P (Kolmakov et al., 2012).

For fluorescence microscopy applications, especially the ones involving powerful lasers (e.g. confocal and all super-resolution microscopy techniques), hydrophilic fluorophores with high quantum yields and improved stability are necessary to visualize specific target molecules or organelles. The best candidates tested herein were Star635P for STED microscopy and AlexaFluor647 for GSDIM/STORM.

The fluorescent dye of choice for biocompatible protein labeling is therefore bright (i.e. high quantum yield and photostability), hydrophilic to reduce nonspecific binding to membranes, 


\section{Discussion}

and, has a red-shifted absorption-emission spectrum, which has been shown to reduce phototoxicity (Schneckenburger et al., 2012). Ideally, it should cross the cell membrane without permeabilization and fluoresce only when tagged to the protein/structure of interest. Even though many fluorophores comply to the first two requirements, the third is more rarely fulfilled. Recently, membrane-permeable silicon-rhodamine (SiR) dyes, in which the oxygen atom of rhodamine has been replaced with silicon, were employed for live cell cytoplasmic stainings (Lukinavičius et al., 2013, 2014; D’Este et al., 2015). However, their performance in terms of photostability, solubility and hydophilicity still need addressing (Kolmakov et al., 2015) .

\subsubsection{Membrane Sheets}

Membrane sheet generation involves the use of a brief ultrasound pulse to remove the cell cortices leaving behind the basal plasma membrane that is adhered to the coverslip together with part of the associated proteins (Holroyd et al., 2002; Lang, 2008; Saka et al., 2014a). This technique allows for fast access to the intracellular side of the plasma membrane and avoids the use of permeabilization (Avery et al., 2000). Therefore, it offers the advantage that the plasma membrane is as close as possible to the state it would have in the cell. However, the are some restrictions imposed by this technique. The lack of membrane curvature is not a natural condition in cells and it arises when the plasma membrane is attached to the coverslip, forcing it to adopt a flat conformation.

The possible artifacts due to the sonication procedure mainly involve the mechanical forces induced in the plasma membrane organization by the ultrasound pulse and the removal of proteins and/or protein assemblies that are loosely interacting with the plasma membrane due to cytosol removal and subsequent washes in buffers. So I sought to minimize the effects caused by possible changes in the $\mathrm{pH}$ and electrostatic charge differences and ionic interactions and I sonicated the cells in a buffer containing the main constituents of the cytosol: monopotassium glutamate at a concentration of $120 \mathrm{mM}$, potassium acetate (20 mM), EGTA $(2 \mathrm{mM})$ to chelate $\mathrm{Ca}^{2+} / \mathrm{Mg}^{2+}$ ions and prevent exocytosis, and HEPES $(20 \mathrm{mM})$ brought to a $\mathrm{pH}$ of 7.2 to mimick the slightly alkaline environment within the cell. However, it is difficult to offer an estimate to which extent the lack of proteins in the buffering system affected the macromolecular assemblies formed by SNAP-25 and syntaxin 1 in STED imaging. As a control of this possible artifact, in GSDIM measurements I employed full cells which were permeabilized to investigate the differences between the 
arrangement formed the wild-type proteins versus the FP chimeras. As in this case the cells were first fixed and then permeabilized, I can assume that a smaller amount of proteins were washed off or extracted.

Membrane sheets require fixation immediately after they have been generated. When incubated for longer, the proteins in the membrane tend to aggregate due to the loss of stabilizing components over time (Frick et al., 2007). As mentioned in Section 4.2.2, the possible cross-linking induced by $\mathrm{Cu}^{2+}$ ions (Link and Tirrell, 2003) is another reason to fix the membrane sheets before carrying out the click reaction.

Other possible controls for sonication-induced artifacts would be either to first fix the cells and then perform sonication or to sonicate them in the presence of a buffer that contains either BSA or a mix of proteins better resembling the cytosol.

\subsubsection{Embedding Techniques}

The plastic embedding of cells has been developed in an attempt to preserve the ultrastructural details of the samples that are measured or analyzed using methods damaging to the integrity of the preparation. For example, the NanoSIMS instrument focuses an ion beam onto the sample which can cause damage to living cells or improperly preserved samples. This would render the imaging results unreliable and full of artifacts.

There are many commercially available polymerizing reagents that can be used to embed biological samples. Embedding procedures work by replacing the water in the preparation with a solution containing reagents that can polymerize under controlled conditions. Ideally, this polymerization reaction should take place as close as possible to the conditions present in living cells: $37^{\circ} \mathrm{C}$ temperature, $\mathrm{pH}$ 7.2-7.4, water as a solvent etc. Another common issue is that few of the organic compounds are soluble in water. The above prerequisites reduce the number of embedding reagent to a handful that accurately preserve biological material.

During routine fluorescence microscopy investigations I performed Mowiol embedding (e.g. Figure 3-15) and also for imaging membrane sheets and thin cell sections at the STED miscroscope (Figure 3-18). Mowiol has been shown to induce imaging artifacts due to spherical aberrations caused by the different refractive index of the Mowiol compaired with the oil and the glass coverslip if a sample depth beyond $10 \mu \mathrm{m}$ is reached (Revelo and Rizzoli, 2015). However these artifacts should be absent in the preparations used in this project, because they involved either surface staining (e.g. for membrane sheets) or $\sim 100 \mathrm{~nm}$ thin sections. 
I embedded in melamine all the GSDIM imaged samples and the $\alpha$-synuclein STED ones. This embedding technique involves the use of the water-soluble melamin monomer. Hence no dehydration step is required, ensuring a good preservation of the cellular structures. For example, melamin is allowed to penetrate the sample at room temperature but requires $40^{\circ} \mathrm{C}$ for polymerization to take place. The embedding procedure requires at least three days until the samples are ready for this sectioning (Revelo and Rizzoli, 2015). Melamin embedding and thin sectioning have been successfully applied to improve the axial resolution in $3 \mathrm{D}$ reconstructions of STED images (Punge et al., 2008).

For NanoSIMS preparations I resorted to the LRWhite embedding procedure because it offers a stronger matrix for high energy beam investigations (Revelo and Rizzoli, 2015). Additionally, the melamin resin is incompatible with the NanoSIMS detection of nitrogen species (refer to discussion in Section 4.3.8; Saka et al., 2014b). LRWhite is an acrylic monomer that polymerizes in a fast and highly exothermic reaction, which requires cooling down to minimize heat-induced damage to the samples and to achieve proper embedding. Sample preservation in LRWhite is more prone to induce artifacts than melamine embedding due to the insolubility of LRWhite in water. This makes it necessary to dehydrate the samples using increasing concentrations of ethanol. The accumulation in the ER of syntaxin 1 (see Figure 3-28 and Figure 3-30), is likely caused by the dehydration procedure prior to LRWhite embedding as well as the heterologous expression of this synaptic protein in BHK cells that lack the protein complement for proper localization of syntaxin 1. Indeed, Munc18-1 is an essential interaction partner expressed only in neurons that acts as a chaperone and helps it localize to the plasma membrane (Han et al., 2011).

\subsubsection{Microscopy Measurements}

The assemblies formed by proteins cannot be accurately investigated in conventional fluorescence microscopy. The lateral resolution has a crucial influence on the apparent sizes of the detected proteins assemblies. For accurate determination of the protein assembly nanostructure, it is necessary to have a resolution at least equal (or better than) half of the diameter of the object investigated, according to the Nyquist-Shannon criterion (Shroff et al., 2008). I therefore employed for imaging state-of-the-art super-resolution microscopes reaching a lateral resolution of $\sim 30 \mathrm{~nm}$ for STED (Göttfert et al., 2013) and $\sim 20 \mathrm{~nm}$ for GSDIM (Fölling et al., 2008). The high resoltion is crucial for correct measurements of the parameters used later in the analysis of protein organization (see Section 4.3.7). 
STED and GSDIM rely on different physical principles and are thus affected by different limitations. In order to overcome these limitations and to obtain a more complete picture, I used both super-resolution techniques.

While GSDIM obviously provides a better resolution, it is more difficult to assess whether the observed spots form a protein cluster (or assembly) or not using this technique (Sengupta et al., 2011; Bar-On et al., 2012). This has to do with the principle behind GSDIM that relies on the individual fluorophore detection and then finding their precise localization. In contrast, STED detects the fluorescence emitted by all fluorophores that are confined in the center of the STED laser beam and are not quenched by it. As a result, it is easier to directly visualize protein assemblies in STED than in the pointillistic methods. The latter require many "ON-OFF" cycles and a high labeling density to reveal enough of the proteins in the assembly to make the distinction between clustered and non-clustered entities.

When comparing the images obtained in STED and in GSDIM, the intensity of the detected spots is not directly comparable because of the different physical principles employed to attain super-resolution. STED the intensity is given by the number of photons detected per pixel, whereas the GSDIM intensity profile describes the number of "ON-OFF" switching events counted in a particular pixel. It is normal for the STED and GSDIM intensity values to be different considering that the measurements were performed with devices that acquire the light emitted by the fluorophores in different temporal and spatial modes.

The three proteins investigated using both of these nanoscopy techniques (SNAP-25, syntaxin 1 , and $\alpha$-synuclein) revealed no significant differences in the sizes or the intensities of their protein assemblies they form in the presence or absence of an FP tag (Figure 3-18 and Figure 3-19). The protein assemblies detected in STED showed a marked difference between the means and the median values of the spot size, but not for the peak intensity. The mean spot sizes for SNAP-25, syntaxin 1, and $\alpha$-synuclein are $\sim 60 \mathrm{~nm}, 50 \mathrm{~nm}$, and $80 \mathrm{~nm}$, respectively; whereas the medians values are $\sim 40 \mathrm{~nm}, 35 \mathrm{~nm}$, and $55 \mathrm{~nm}$. The median values are much closer to the spot sizes determined in GSDIM microscopy: $\sim 35 \mathrm{~nm}$ for SNAP-25, $\sim 30 \mathrm{~nm}$ for syntaxin 1, and $\sim 35 \mathrm{~nm}$ for $\alpha$-synuclein assemblies. This good accordance with GSDIM data validates the use of medians for STED data analysis. Moreover, this observation implies that the STED dataset contains a few large protein assemblies that skew the mean towards higher values.

In previous studies, syntaxin 1 has been reported to form protein clusters with 75 syntaxin copies and a diameter of 50-60 nm and (Sieber et al., 2007). However these studies used 
antibodies to detect syntaxin and the resolution reached only $50 \mathrm{~nm}$ for the STED measurements. Therefore the differences between these results and my own could be related to artifacts induced by the larger antibody probes and/or the lower resolution of the microscope. A more recent study also employed antibodies against syntaxin 1 and SNAP-25 to investigate the clusters they form using a dSTORM setup (Bar-On et al., 2012). In this study Ashery and collaborators found a mean cluster diameter of $\sim 93 \mathrm{~nm}$ for syntaxin 1, with a very dense central area and loose assemblies on the margins, while larger clusters of $\sim 130 \mathrm{~nm}$ (mean value) were detected for SNAP-25. The considerable differences of these previous report compared to the data acquired using ncAA is probably due to the the antibody tags used for detecting both SNAP-25 and syntaxin 1. The bivalent antibody molecules are likely to induce artificial clustering.

\subsubsection{Analysis of Protein Organization}

The images acquired using the above-mentioned resolution techniques were analyzed to reveal putative differences between non-tagged proteins and their FP-fusions. In this analysis, protein organization was assayed by first selecting the spots from regions of interest (e.g. cells or membrane sheets), and then sorting them to remove single-molecule spots (see Section 2.14.3). Then a line scan was performed to derive the FWHM (spot size), peak intensity and total spot intensity. After the removal of single-molecule spots, each spot is presumably a macromolecular assembly, containing multiple copies of the protein of interest.

The total spot intensity value might not be particularly useful because it depends not only on the brightness of the particular spot, but also on its apparent size. In addition, it is also dependent on the technical aspects of the measurements, especially the background. The latter influences the baseline of the measurement, which in turn affects the total area under the intensity curve and thereby introduces a higher variability in the total intensity results. However, for dim spots, which otherwise tend to be overlooked, the total intensity is a valuable parameter.

One conceptual caveat is the fact in the data analysis all spots were assumed to be circular. This could influence the results for the line-fitting process applied to the spots. To continue with, it could also induce a bias and lead to measurement errors for the spot size. But in such a case, the peak intensity determination would be least affected and should be used as a control for validating the spot size measurements. 


\subsubsection{NanoSIMS Measurements}

The NanoSIMS instrument does not detect all the particles it sputters mainly because most of them are not electrically charged. As a result, not all the particles in the sample can be captured by the ion lens, so they do not reach the detectors. To continue with, different molecules and atoms form ions with variable efficiency. This effect is mostly influenced by the matrix in which the atoms and molecules are placed. Even between isotopes of the same element there are differences in ionization that arise from the different atomic weight of the isotopes. This limits to some extent the sensitivity of the NanoSIMS technique and requires the use of relatively high amounts of isotopic labels within the sample to ensure accurate detection (Lechene et al., 2006; Hoppe et al., 2013). To overcome this technical issue, the probes designed for SPILL were highly enriched in isotopes normally found in low (e.g. ${ }^{15} \mathrm{~N}$ ) to trace (e.g. ${ }^{19} \mathrm{~F}$ ) amounts in cells.

Sputtering the sample with energetic ion beams can lead to heat-induced damage. Aside from this, due to sample stage movements, it is recommendable to subdivide the image acquisition process into thinner planes, which later allow the correction of the possible drifts. In practice, distortions are minimized by using a finely tuned temperature control and a vibration proof setting of the instrument.

In order to have a good balance between detection sensitivity and resolution, the sputtering parameters and acquisition times were set to reach $\sim 200 \mathrm{~nm}$ in lateral and $\sim 20 \mathrm{~nm}$ in axial resolution. I embedded the NanoSIMS samples in LRWhite (refer to Section 4.3.5 for details on embedding procedures) because the melamine monomer $\left(\mathrm{C}_{3} \mathrm{H}_{6} \mathrm{~N}_{6}\right)$ contains a high amount of nitrogen atoms. The latter affects the ${ }^{15} \mathrm{~N}$ to ${ }^{14} \mathrm{~N}$ ratio measurements by increasing the nitrogen background in the sample. Thus, such an embedding medium masks the specific signal coming from the ${ }^{15} \mathrm{~N}$-labeled proteins. 


\section{CONCLUSIONS AND OUTLOOK}

In this project, I successfully employed the incorporation of the non-canonical amino acid propargyl-L-lysine (PRK) to label various proteins of interest either for fluorescence superresolution microscopy investigations or for nanoscale secondary ion mass spectrometry applications.

Amongst the six different ncAAs tested herein, PRK fulfilled the goals of this project best due to its almost-quantitative and highly specific labeling reaction with azide derivatives that are optimized either for fluorescence nanoscopy or for isotopic imaging. PRK is a biocompatible and bioorthogonal compound. It differs from the canonical amino acid lysine by only a few atoms, so using it as a protein tag induces minimal perturbations to the protein of interest. This constitutes a good basis for proof-of-principle experiments into specific protein organization and turnover.

In summary, these results support the use of non-canonical amino acids, in particular PRK, for labeling proteins.

One major outlook expanding the genetic code involves the possibility to incorporate more than one ncAA and to label multiple proteins in one cell. For this, other codons except the Amber nonsense one should be reassigned or made available for ncAA incorporation. One such possibility would be the use of quadruplet codons for the genetic encoding of ncAAs, which would open the way for multicolor protein labeling using ncAAs.

Another technical improvement would be the reliable implementation of the copper-free click chemistry for labeling both fixed cells and especially live ones. With further optimization of the trade-off between reactivity and specificity, copper-free click reaction should become a good alternative to the copper-catalyzed click reaction. Another awaited improvement is the development of clickable probes (e.g. Si-rhodamine dyes) that can cross the cell membrane and label intracellular proteins in the absence of permeabilizing agents. Taken together, ncAAs amenable to copper-free click chemistry and cell-permeable probes should provide insight into the dynamics of the protein turnover and assembly formation. 


\subsection{Labeling of Proteins for Super-Resolution Light Microscopy}

In this first part of my project, I tested a representative population of neuronal proteins for possible artifacts induced by FP tagging. From the cohort of 26 proteins, only two proteins were considerably affected in the organization of their assemblies: PIPKI $\gamma$ and VAMP4. Both their size and the intensities of their spots showed highly significant changes but, surprisingly, FP tagging had opposite effects on their organization. Other 5 proteins show significant but small differences either in the brightness or in the sizes of the multimers they form.

To conclude, no coherent trend can be observed when comparing the organization of proteins with or without FP tags. FP tagging affects both cytosolic and membrane-attached proteins to variable extents. Of notice is the fact that only tail-anchored proteins and cytosolic and/or peripheral membrane proteins were affected by FP tagging. Interestingly, none of the multiple pass membrane proteins, such as 5HT1a or synaptophysin, nor the proteins attached to the membrane through fatty acid chains, like SNAP-25, showed any significant differences.

Therefore, these results come as a validation of the studies involving FPs and suggest that inspite of the relatively large size of an FP tag, it generates no changes in the organization of the large majority of proteins. Nevertheless, care has to be taken for proteins such as PIPKI $\gamma$ and VAMP4, which show perturbed organization upon FP-tagging. For such proteins, smaller tags such as ncAAs are more suitable.

The method described here, namely ncAA incorporation and click reaction with appropriate fluorescent dyes, can be applied to any protein of interest (in the presence and absence of an FP tag) and used as a platform to test for possible changes in protein organization. As discussed above, this method could also be applied to live cells if further improvements in copper-free click chemistry and fluorescent dye properties will be accomplished.

One limitation of this study is the fact that the majority of the investigated proteins are enriched or specifically expressed in neurons. It would be informative to compare the results obtained here with data obtained from neuronal studies. This procedure will require further optimization of the transfection and non-canonical amino acid incorporation technique.

An interesting research line would be to elucidate the reasons behind the drastic differences of the assemblies formed by PIPKI $\gamma$ when FP tagged. To answer this, other PIPKI $\gamma$ splice 
variants can be tested in a similar assay and see whether the proximity of the FP to important domains could be a contributing factor. A similar question can be asked at a protein family level: if VAMP4 shows dramatic differences, while VAMP2 doesn't, how do the rest of the VAMPs perform upon FP tagging?

A more technical outlook would be to count the fluorophores in each detected spot (i.e. protein assembly) using the recently described technique that allows their mapping (Ta et al., 2015). This could offer insight not only on the size and number of proteins in an assembly, but also on the density or level of compaction with the assembly.

\subsection{Labeling of Proteins for NanoSIMS}

In the second part of this study, I established the SPILL method for genetically labeling proteins for NanoSIMS. I employed the clickable amino acid PRK to specifically mark three proteins of interest: syntaxin 1, SNAP-25 and syntaxin 13. The labeling reaction with SPILL probes was reliable: SK155 ensured sensitive detection of the proteins of interest based on the ${ }^{19} \mathrm{~F}$ isotopic marker, while TriazNF1 enabled the quantification of protein numbers from the ${ }^{15} \mathrm{~N}$ to ${ }^{14} \mathrm{~N}$ ratio. Using either of these probes I could visualize precisely the proteins of interest in both fluorescence microscopy and NanoSIMS, so both of these probes render optional the use of fluorescence imaging.

SK155 enabled the analysis of specific protein turnover in the context of general protein metabolism. A result that raises major concerns is the unreliability of the quantitative correlation between NanoSIMS and fluorescence microscopy. This observation is likely due to technique-related artifacs, different axial resolution profiles of the optical microscopes versus isotopic detectors, as well as the errors induced by misalignments of the images obtained in the two different methods. The main conclusion of this technical investigation is that proteins labeled in SPILL are more reliably characterized in turnover studies directly in NanoSIMS, than if they were immunolabeled and correlation would be performed.

Therefore, the correlation between protein turnover, indicated by the ${ }^{15} \mathrm{~N}$ to ${ }^{14} \mathrm{~N}$ ratio, and the ${ }^{19} \mathrm{~F}$ signal provided a much more reliable correlation. Interestingly, SNAP-25 and syntaxin 13 exhibited a significant tendency to be excluded from cell regions enriched in ${ }^{15} \mathrm{~N}$, while syntaxin 1 showed no preference. A worthwhile perspective would involve further turnover studies with different ${ }^{15} \mathrm{~N}$ and PRK labeling time points in an attempt to put these observations into context. Another interesting research direction would be to 
investigate the turnover of SNAP-25 and syntaxin 13 in a biologically relevant system, such as neuronal cultures.

As a technical outlook, ncAAs directly enriched in stable isotopes like ${ }^{15} \mathrm{~N}$ or ${ }^{19} \mathrm{~F}$ may be tested. However, there are considerable concerns with regard to the background coming from the unincorporated ncAA. Another technical aspect that requires improvement is the sample embedding for NanoSIMS. Ideally, an embedding reagent that does not require prior dehydration should be employed to better preserve the cellular architecture. One such option would be to perform high-pressure freezing followed by cryosubstitution. This would hopefuly also improve the accuracy of the correlation between specific protein turnover and general protein metabolism.

In summary, SPILL offers the unprecedented possibility to directly visualize in NanoSIMS the protein of interest in the larger context of the general protein turnover. In principle any isotopic probe can be added. These proof-of-principle investigations imply that ncAAs and their click reaction with isotopic probes can be used in NanoSIMS in an analogous manner to the genetically encoded FPs in fluorescence microscopy. 


\section{BIBLIOGRAPHY}

Abbe, E. (1873). Beitrage zur Theorie des Mikroskops und der mikroskopischen Wahrnehmung. Archiv für mikroskopische Anatomie, 9(1), 413-418.

Abbe, E. (1881). On the estimation of aperture in the microscope. Journal of the Royal Microscopical Society, 1(3), 388-423.

Abbe, E. (1883). The relation of aperture and power in the microscope. Journal of the Royal Microscopical Society, 3(6), 790-812.

Adams, S. R., Campbell, R. E., Gross, L. A., Martin, B. R., Walkup, G. K., Yao, Y., ... Tsien, R. Y. (2002). New biarsenical ligands and tetracysteine motifs for protein labeling in vitro and in vivo: synthesis and biological applications. Journal of the American Chemical Society, 124(21), 6063-6076.

Adams, T. S., and Sterner, R. W. (2000). The effect of dietary nitrogen content on trophic level ${ }^{15} \mathrm{~N}$ enrichment. Limnology and Oceanography, 45(3), 601-607.

Agard, N. J., Prescher, J. A., and Bertozzi, C. R. (2004). A strain-promoted [3 + 2] azidealkyne cycloaddition for covalent modification of biomolecules in living systems. Journal of the American Chemical Society, 126(46), 15046-15047.

Alder, K., and Stein, G. (1933). Über das abgestufte Additionsvermögen von ungesättigten Ringsystemen. II. Justus Liebigs Annalen der Chemie, 501(1), 1-48.

Alder, K. Von, and Stein, G. (1931). Über das abgestufte Additionsvermögen von ungesättigten Ringsystemen. Justus Liebigs Annalen der Chemie, 485(1912), 211.

Ambrogelly, A., Palioura, S., and Söll, D. (2007). Natural expansion of the genetic code. Nature Chemical Biology, 3(1), 29-35.

Anderson, J. C., Wu, N., Santoro, S. W., Lakshman, V., King, D. S., and Schultz, P. G. (2004). An expanded genetic code in mammalian cells with a functional quadruplet codon. Proceedings of the National Academy of Sciences of the United States of America, 101(20), 7566-7571.

Anderton, C. R., Lou, K., Weber, P. K., Hutcheon, I. D., and Kraft, M. L. (2011). Correlated AFM and NanoSIMS imaging to probe cholesterol-induced changes in phase behavior and non-ideal mixing in ternary lipid membranes. Biochimica et Biophysica Acta, 1808(1), 307-315.

Angelo, M., Bendall, S. C., Finck, R., Hale, M. B., Hitzman, C., Borowsky, A. D., ... Nolan, G. P. (2014). Multiplexed ion beam imaging of human breast tumors. Nature Medicine, $20,436-442$.

Annibale, P., Vanni, S., Scarselli, M., Rothlisberger, U., and Radenovic, A. (2011a). Identification of clustering artifacts in photoactivated localization microscopy. Nature methods, 8(7), 527-528. 
Annibale, P., Vanni, S., Scarselli, M., Rothlisberger, U., and Radenovic, A. (2011b). Quantitative photo activated localization microscopy: unraveling the effects of photoblinking. PloS One, 6(7), e22678.

Antonin, W., Riedel, D., and von Mollard, G. F. (2000). The SNARE Vtila-beta is localized to small synaptic vesicles and participates in a novel SNARE complex. The Journal of Neuroscience, 20(15), 5724-5732.

Avery, J., Ellis, D. J., Lang, T., Holroyd, P., Riedel, D., Henderson, R. M., ... Jahn, R. (2000). A cell-free system for regulated exocytosis in PC12 cells. The Journal of Cell Biology, 148(2), 317-324.

Axelrod, D. (1981). Cell-substrate contacts illuminated by total internal reflection fluorescence. Journal of Cell Biology, 89(1), 141-145.

Baddeley, D., Jayasinghe, I. D., Cremer, C., Cannell, M. B., and Soeller, C. (2009). Lightinduced dark states of organic fluochromes enable $30 \mathrm{~nm}$ resolution imaging in standard media. Biophysical Journal, 96(2), L22-L24.

Bar-On, D., Wolter, S., van de Linde, S., Heilemann, M., Nudelman, G., Nachliel, E., ... Ashery, U. (2012). Super-resolution imaging reveals the internal architecture of nanosized syntaxin clusters. The Journal of Biological Chemistry, 287(32), 27158-27167.

Bates, M., Huang, B., Dempsey, G. T., and Zhuang, X. (2007). Multicolor super-resolution imaging with photo-switchable fluorescent probes. Science, 317(5845), 1749-1753.

Benesch, R. E., and Benesch, R. (1953). Enzymatic removal of oxygen for polarography and related methods. Science, 118(3068), 447-448.

Benninghoven, A., Rüdenauer, F., and Werner, H. (1987). Secondary Ion Mass Spectrometry: Basic Concepts, Instrumental Aspects, Applications, and Trends. New York, NY: Wiley.

Betzig, E., Patterson, G. H., Sougrat, R., Lindwasser, O. W., Olenych, S., Bonifacino, J. S., ... Hess, H. F. (2006). Imaging intracellular fluorescent proteins at nanometer resolution. Science, 313(5793), 1642-1645.

Bianco, A., Townsley, F. M., Greiss, S., Lang, K., and Chin, J. W. (2012). Expanding the genetic code of Drosophila melanogaster. Nature Chemical Biology, 8(9), 748-750.

Blackman, M. L., Royzen, M., and Fox, J. M. (2008). Tetrazine ligation: Fast bioconjugation based on inverse-electron-demand Diels-Alder reactivity. Journal of the American Chemical Society, 130(41), 13518-13519.

Blight, S. K., Larue, R. C., Mahapatra, A., Longstaff, D. G., Chang, E., Zhao, G., ... Krzycki, J. A. (2004). Direct charging of tRNA(CUA) with pyrrolysine in vitro and in vivo. Nature, 431(7006), 333-335.

Boger, D. L. (1986). Diels-Alder reactions of heterocyclic aza dienes. Scope and applications. Chemical Reviews, 86(5), 781-794.

Boyce, M., and Bertozzi, C. R. (2011). Bringing chemistry to life. Nature Methods, 8(8), 638642.

Bright, F. V, and Munson, C. A. (2003). Time-resolved fluorescence spectroscopy for illuminating complex systems. Analytica Chimica Acta, 500(1-2), 71-104.

Bückers, J., Wildanger, D., Vicidomini, G., Kastrup, L., and Hell, S. W. (2011). Simultaneous multi-lifetime multi-color STED imaging for colocalization analyses. Optics Express, 
19(4), 3130-3143.

Campos, C., Kamiya, M., Banala, S., Johnsson, K., and González-Gaitán, M. (2011). Labelling cell structures and tracking cell lineage in zebrafish using SNAP-tag. Developmental Dynamics, 240(4), 820-827.

Chalfie, M., Tu, Y., Euskirchen, G., Ward, W. W., and Prasher, D. C. (1994). Green fluorescent protein as a marker for gene expression. Science, 263(5148), 802-805.

Chen, H., Bernstein, B. W., and Bamburg, J. R. (2000). Regulating actin-filament dynamics in vivo. Trends in Biochemical Sciences, 25(1), 19-23.

Chen, P. R., Groff, D., Guo, J., Ou, W., Cellitti, S., Geierstanger, B. H., and Schultz, P. G. (2009). A facile system for encoding unnatural amino acids in mammalian cells. Angewandte Chemie International Edition, 48(22), 4052-4055.

Chen, Y., Müller, J. D., Ruan, Q., and Gratton, E. (2002). Molecular brightness characterization of EGFP in vivo by fluorescence fluctuation spectroscopy. Biophysical Journal, 82(1), 133-144.

Chin, J. W. (2003). An Expanded Eukaryotic Genetic Code. Science, 301(5635), 964-967.

Chin, J. W., Cropp, T. A., Chu, S., Meggers, E., and Schultz, P. G. (2003). Progress toward an expanded eukaryotic genetic code. Chemistry and Biology, 10(6), 511-519.

Chin, J. W., Santoro, S. W., Martin, A. B., King, D. S., Wang, L., and Schultz, P. G. (2002). Addition of p-azido-L-phenylalanine to the genetic code of Escherichia coli. Journal of the American Chemical Society, 124(31), 9026-9027.

Conway, K. A., Harper, J. D., and Lansbury, P. T. (1998). Accelerated in vitro fibril formation by a mutant alpha-synuclein linked to early-onset Parkinson disease. Nature Medicine, 4(11), 1318-1320.

Conway, K. A., Lee, S. J., Rochet, J. C., Ding, T. T., Williamson, R. E., and Lansbury, P. T. (2000). Acceleration of oligomerization, not fibrillization, is a shared property of both alpha-synuclein mutations linked to early-onset Parkinson's disease: implications for pathogenesis and therapy. Proceedings of the National Academy of Sciences of the United States of America, 97(2), 571-576.

Cormack, B. P., Valdivia, R. H., and Falkow, S. (1996). FACS-optimized mutants of the green fluorescent protein (GFP). Gene, 173(1), 33-38.

Cowie, D. B., and Cohen, G. N. (1957). Biosynthesis by Escherichia coli of active altered proteins containing selenium instead of sulfur. Biochimica et Biophysica Acta, 26(2), $252-261$.

Crick, F. H. C. (1968). The origin of the genetic code. Journal of Molecular Biology, 38, 367379.

D’Este, E., Kamin, D., Göttfert, F., El-Hady, A., and Hell, S. W. (2015). STED nanoscopy reveals the ubiquity of subcortical cytoskeleton periodicity in living neurons. Cell Reports, 10(8), 1246-1251.

Daugherty, A., Becker, N. N., Scherrer, L. A., Sobel, B. E., Ackerman, J. J., Baynes, J. W., and Thorpe, S. R. (1989). Non-invasive detection of protein metabolism in vivo by NMR spectroscopy. Application of a novel ${ }^{19} \mathrm{~F}$-containing residualizing label. The Biochemical Journal, 264, 829-835. 
Davis, L., and Chin, J. W. (2012). Designer proteins: applications of genetic code expansion in cell biology. Nature Reviews. Molecular Cell Biology, 13(3), 168-182.

Deák, F., Xu, Y., Chang, W. P., Dulubova, I., Khvotchev, M., Liu, X., ... Rizo, J. (2009). Munc18-1 binding to the neuronal SNARE complex controls synaptic vesicle priming. The Journal of Cell Biology, 184(5), 751-764.

Debets, M. F., van Berkel, S. S., Schoffelen, S., Rutjes, F. P. J. T., van Hest, J. C. M., and van Delft, F. L. (2010). Aza-dibenzocyclooctynes for fast and efficient enzyme PEGylation via copper-free (3+2) cycloaddition. Chemical Communications, 46(1), 97-99.

Dempsey, G. T., Vaughan, J. C., Chen, K. H., Bates, M., and Zhuang, X. (2011). Evaluation of fluorophores for optimal performance in localization-based super-resolution imaging. Nature Methods, 8(12), 1027-1036.

Denzer, A. J., Nabholz, C. E., and Spiess, M. (1995). Transmembrane orientation of signalanchor proteins is affected by the folding state but not the size of the N-terminal domain. The EMBO Journal, 14(24), 6311-6317.

Devaraj, N. K., Hilderbrand, S., Upadhyay, R., Mazitschek, R., and Weissleder, R. (2010). Bioorthogonal turn-on probes for imaging small molecules inside living cells. Angewandte Chemie International Edition, 49(16), 2869-2872.

Di Paolo, G., Pellegrini, L., Letinic, K., Cestra, G., Zoncu, R., Voronov, S., ... De Camilli, P. (2002). Recruitment and regulation of phosphatidylinositol phosphate kinase type 1 gamma by the FERM domain of talin. Nature, 420(6911), 85-89.

Dieterich, D. C., Hodas, J. J. L., Gouzer, G., Shadrin, I. Y., Ngo, J. T., Triller, A., ... Schuman, E. M. (2010). In situ visualization and dynamics of newly synthesized proteins in rat hippocampal neurons. Nature Neuroscience, 13(7), 897-905.

Dieterich, D. C., Lee, J. J., Link, A. J., Graumann, J., Tirrell, D. A., and Schuman, E. M. (2007). Labeling, detection and identification of newly synthesized proteomes with bioorthogonal non-canonical amino-acid tagging. Nature Protocols, 2(3), 532-540.

Donnert, G., Eggeling, C., and Hell, S. W. (2007). Major signal increase in fluorescence microscopy through dark-state relaxation. Nature Methods, 4(1), 81-86.

Doughman, R. L., Firestone, A. J., Wojtasiak, M. L., Bunce, M. W., and Anderson, R. A. (2003). Membrane ruffling requires coordination between type Ialpha phosphatidylinositol phosphate kinase and Rac signaling. The Journal of Biological Chemistry, 278(25), 23036-23045.

Du, H. N., Tang, L., Luo, X. Y., Li, H. T., Hu, J., Zhou, J. W., and Hu, H. Y. (2003). A peptide motif consisting of glycine, alanine, and valine is required for the fibrillization and cytotoxicity of human $\alpha$-synuclein. Biochemistry, 42(29), 8870-8878.

Eggeling, C., Willig, K. I., Sahl, S. J., and Hell, S. W. (2015). Lens-based fluorescence nanoscopy. Quarterly Reviews of Biophysics, 48(02), 178-243.

Ellington, A. D., and Szostak, J. W. (1990). In vitro selection of RNA molecules that bind specific ligands. Nature, 346(6287), 818-822.

Fölling, J., Bossi, M., Bock, H., Medda, R., Wurm, C. A., Hein, B., ... Hell, S. W. (2008). Fluorescence nanoscopy by ground-state depletion and single-molecule return. Nature Methods, 5(11), 943-945. 
Fornasiero, E. F., and Opazo, F. (2015). Super-resolution imaging for cell biologists: concepts, applications, current challenges and developments. Bioessays, 37(4), 436-451.

Frick, M., Schmidt, K., and Nichols, B. J. (2007). Modulation of lateral diffusion in the plasma membrane by protein density. Current Biology, 17(5), 462-467.

Frisz, J. F., Lou, K., Klitzing, H. A., Hanafin, W. P., Lizunov, V., Wilson, R. L., ... Kraft, M. L. (2013). Direct chemical evidence for sphingolipid domains in the plasma membranes of fibroblasts. Proceedings of the National Academy of Sciences of the United States of America, 110(8), E613-622.

Frolova, L., Le Goff, X., Rasmussen, H. H., Cheperegin, S., Drugeon, G., Kress, M., ... Philippe, M. (1994). A highly conserved eukaryotic protein family possessing properties of polypeptide chain release factor. Nature, 372(6507), 701-703.

Frommer, W. B., Davidson, M. W., and Campbell, R. E. (2009). Genetically encoded biosensors based on engineered fluorescent proteins. Chemical Society Reviews, 38(10), 2833-2841.

Furter, R. (1998). Expansion of the genetic code: site-directed p-fluoro-phenylalanine incorporation in Escherichia coli. Protein Science, 7(2), 419-426.

Galbraith, C. G., and Galbraith, J. A. (2011). Super-resolution microscopy at a glance. Journal of Cell Science, 124(10), 1607-1611.

Gautier, A., Juillerat, A., Heinis, C., Corrêa, I. R., Kindermann, M., Beaufils, F., and Johnsson, K. (2008). An engineered protein tag for multiprotein labeling in living cells. Chemistry and Biology, 15(2), 128-136.

Gautier, A., Nakata, E., Lukinavičius, G., Tan, K. T., and Johnsson, K. (2009). Selective cross-linking of interacting proteins using self-labeling tags. Journal of the American Chemical Society, 131(49), 17954-17962.

Gerber, S. H., Rah, J.-C., Min, S.-W., Liu, X., de Wit, H., Dulubova, I., ... Südhof, T. C. (2008). Conformational switch of syntaxin-1 controls synaptic vesicle fusion. Science, $321(5895), 1507-1510$.

Giasson, B. I., Murray, I. V. J., Trojanowski, J. Q., and Lee, V. M. Y. (2001). A hydrophobic stretch of 12 amino acid residues in the middle of $\alpha$-synuclein is essential for filament assembly. Journal of Biological Chemistry, 276(4), 2380-2386.

Gonzalez, P., Robinet, P., Charpentier, S., Mollet, L., Normand, T., Dubois, M., and Legrand, A. (2009). Apoptotic activity of a nuclear form of mitogaligin, a cell death protein. Biochemical and Biophysical Research Communications, 378(4), 816-820.

Göttfert, F., Wurm, C. A., Mueller, V., Berning, S., Cordes, V. C., Honigmann, A., and Hell, S. W. (2013). Coaligned dual-channel STED nanoscopy and molecular diffusion analysis at $20 \mathrm{~nm}$ resolution. Biophysical Journal, 105(1), L01-3.

Greenbaum, E. A., Graves, C. L., Mishizen-Eberz, A. J., Lupoli, M. A., Lynch, D. R., Englander, S. W., ... Giasson, B. I. (2005). The E46K mutation in $\alpha$-synuclein increases amyloid fibril formation. Journal of Biological Chemistry, 280(9), 7800-7807.

Greiss, S., and Chin, J. W. (2011). Expanding the genetic code of an animal. Journal of the American Chemical Society, 133(36), 14196-14199.

Griffin, B. A., Adams, S. R., and Tsien, R. Y. (1998). Specific covalent labeling of 
recombinant protein molecules inside live cells. Science, 281(5374), 269-272.

Guo, J.-K., Cheng, E.-C., Wang, L., Swenson, E. S., Ardito, T. A., Kashgarian, M., ... Krause, D. S. (2007). The commonly used beta-actin-GFP transgenic mouse strain develops a distinct type of glomerulosclerosis. Transgenic Research, 16(6), 829-834.

Gustafsson, M. G. (2000). Surpassing the lateral resolution limit by a factor of two using structured illumination microscopy. Journal of Microscopy, 198(2), 82-87.

Ha, T., and Tinnefeld, P. (2012). Photophysics of fluorescent probes for single-molecule biophysics and super-resolution imaging. Annual Review of Physical Chemistry, 63(1), 595-617.

Haas, J., Park, E. C., and Seed, B. (1996). Codon usage limitation in the expression of HIV-1 envelope glycoprotein. Current Biology, 6(3), 315-324.

Hackmann, Y., Graham, S. C., Ehl, S., Höning, S., Lehmberg, K., Aricò, M., ... Griffiths, G. M. (2013). Syntaxin binding mechanism and disease-causing mutations in Munc18-2. Proceedings of the National Academy of Sciences of the United States of America, 110(47), E4482-4491.

Halemani, N. D., Bethani, I., Rizzoli, S. O., and Lang, T. (2010). Structure and dynamics of a two-helix SNARE complex in live cells. Traffic, 11(3), 394-404.

Hamamoto, Y., Akashi, T., Inada, A., Bonner-Weir, S., and Weir, G. C. (2010). Lack of evidence for recipient precursor cells replenishing $\beta$-cells in transplanted islets. Cell Transplantation, 19(12), 1563-1572.

Hammond, J. W., Blasius, T. L., Soppina, V., Cai, D., and Verhey, K. J. (2010). Autoinhibition of the kinesin-2 motor KIF17 via dual intramolecular mechanisms. Journal of Cell Biology, 189(6), 1013-1025.

Han, G. A., Malintan, N. T., Saw, N. M. N., Li, L., Han, L., Meunier, F. A., ... Sugita, S. (2011). Munc18-1 domain-1 controls vesicle docking and secretion by interacting with syntaxin-1 and chaperoning it to the plasma membrane. Molecular Biology of the Cell, 22(21), 4134-4149.

Heilemann, M., Van De Linde, S., Schüttpelz, M., Kasper, R., Seefeldt, B., Mukherjee, A., ... Sauer, M. (2008). Subdiffraction-resolution fluorescence imaging with conventional fluorescent probes. Angewandte Chemie International Edition, 47(33), 6172-6176.

Hell, S. W. (2003). Toward fluorescence nanoscopy. Nature Biotechnology, 21(11), 13471355.

Hell, S. W. (2007). Far-field optical nanoscopy. Science, 316(5828), 1153-1158.

Hell, S. W. (2009). Microscopy and its focal switch. Nature Methods, 6(1), 24-32.

Herschel, W. (1843). On a case of superficial colour presented by a homogeneous liquid internally colourless. Proceedings of the Royal Society of London, 5(0), 547-547.

Hess, S. T., Girirajan, T. P. K., and Mason, M. D. (2006). Ultra-high resolution imaging by fluorescence photoactivation localization microscopy. Biophysical Journal, 91(11), $4258-4272$.

Hino, N., Sakamoto, K., and Yokoyama, S. (2012). Unnatural Amino Acids. (L. Pollegioni and S. Servi, Eds.) (Vol. 794). Totowa, NJ: Humana Press. 
Hodgson, L., Qiu, W., Dong, C., and Henderson, A. J. (2000). Use of green fluorescent protein-conjugated beta-actin as a novel molecular marker for in vitro tumor cell chemotaxis assay. Biotechnology Progress, 16(6), 1106-1114.

Holmes, K. C., Popp, D., Gebhard, W., and Kabsch, W. (1990). Atomic model of the actin filament. Nature, 347(6288), 44-49.

Holroyd, P., Lang, T., Wenzel, D., De Camilli, P., and Jahn, R. (2002). Imaging direct, dynamin-dependent recapture of fusing secretory granules on plasma membrane lawns from PC12 cells. Proceedings of the National Academy of Sciences of the United States of America, 99(26), 16806-16811.

Hong, V., Steinmetz, N. F., Manchester, M., and Finn, M. G. (2011). Labeling live cells by copper-catalyzed alkyne-azide click chemistry. Bioconjugate Chemistry, 21(10), 19121916.

Hoopmann, P., Punge, A., Barysch, S. V, Westphal, V., Bückers, J., Opazo, F., ... Rizzoli, S. O. (2010). Endosomal sorting of readily releasable synaptic vesicles. Proceedings of the National Academy of Sciences of the United States of America, 107(44), 19055-19060.

Hoppe, P., Cohen, S., and Meibom, A. (2013). NanoSIMS: Technical Aspects and Applications in Cosmochemistry and Biological Geochemistry. Geostandards and Geoanalytical Research, 37(2), 111-154.

Hu, J., Yuan, Q., Kang, X., Qin, Y., Li, L., Ha, Y., and Wu, D. (2015). Resolution of structure of PIP5K1A reveals molecular mechanism for its regulation by dimerization and dishevelled. Nature Communications, 6, 8205.

$\mathrm{Hu}, \mathrm{Y} ., \mathrm{Qu}, \mathrm{L}$. , and Schikorski, T. (2008). Mean synaptic vesicle size varies among individual excitatory hippocampal synapses. Synapse, 62(12), 953-957.

Hua, Y., Sinha, R., Thiel, C. S., Schmidt, R., Hüve, J., Martens, H., ... Klingauf, J. (2011). A readily retrievable pool of synaptic vesicles. Nature Neuroscience, 14(7), 833-839.

Huang, B. (2010). Super-resolution optical microscopy: multiple choices. Current Opinion in Chemical Biology, 14(1), 10-14.

Hughes, L. D., Rawle, R. J., and Boxer, S. G. (2014). Choose your label wisely: water-soluble fluorophores often interact with lipid bilayers. PLoS One, 9(2), e87649.

Huisgen, R. (1963). 1,3-Dipolar cycloadditions. Past and future. Angewandte Chemie International Edition, 2(10), 565-598.

Inouye, S., and Tsuji, F. I. (1994). Aequorea green fluorescent protein. Expression of the gene and fluorescence characteristics of the recombinant protein. FEBS Letters, 341(2-3), 277-280.

Ishihara, H., Shibasaki, Y., Kizuki, N., Wada, T., Yazaki, Y., Asano, T., and Oka, Y. (1998). Type I phosphatidylinositol-4-phosphate 5-kinases. Cloning of the third isoform and deletion/substitution analysis of members of this novel lipid kinase family. Journal of Biological Chemistry, 273(15), 8741-8748.

Jain, R. K., Joyce, P. B., Molinete, M., Halban, P. A., and Gorr, S. U. (2001). Oligomerization of green fluorescent protein in the secretory pathway of endocrine cells. The Biochemical Journal, 360(3), 645-649.

Johnson, J. A., Lu, Y. Y., Van Deventer, J. A., and Tirrell, D. A. (2010). Residue-specific 
incorporation of non-canonical amino acids into proteins: recent developments and applications. Current Opinion in Chemical Biology, 14(6), 774-780.

Kabatas, S., Vreja, I. C., Saka, S. K., Höschen, C., Kröhnert, K., Opazo, F., ... Diederichsen, U. (2015). A contamination-insensitive probe for imaging specific biomolecules by secondary ion mass spectrometry. Chemical Communications, 51(67), 13221-13224.

Keppler, A., Gendreizig, S., Gronemeyer, T., Pick, H., Vogel, H., and Johnsson, K. (2003). A general method for the covalent labeling of fusion proteins with small molecules in vivo. Nature Biotechnology, 21(1), 86-89.

Kisselev, L., Ehrenberg, M., and Frolova, L. (2003). Termination of translation: interplay of mRNA, rRNA and release factors? The EMBO Journal, 22(2), 175-182.

Köhrer, C., Sullivan, E. L., and RajBhandary, U. L. (2004). Complete set of orthogonal 21st aminoacyl-tRNA synthetase-amber, ochre and opal suppressor tRNA pairs: concomitant suppression of three different termination codons in an mRNA in mammalian cells. Nucleic Acids Research, 32(21), 6200-6211.

Kolb, H. C., Finn, M. G., and Sharpless, K. B. (2001). Click chemistry: diverse chemical function from a few good reactions. Angewandte Chemie International Edition, 40(11), 2004-2021.

Kolmakov, K., Belov, V. N., Bierwagen, J., Ringemann, C., Müller, V., Eggeling, C., and Hell, S. W. (2010). Red-emitting rhodamine dyes for fluorescence microscopy and nanoscopy. Chemistry, 16(1), 158-166.

Kolmakov, K., Hebisch, E., Wolfram, T., Nordwig, L. A., Wurm, C. A.., Ta, H., ... Hell, S. W. (2015). Far-red emitting fluorescent dyes for optical nanoscopy: fluorinated siliconrhodamines (SiRF dyes) and phosphorylated oxazines. Chemistry, 21(38), 13344-13356.

Kolmakov, K., Wurm, C. A., Hennig, R., Rapp, E., Jakobs, S., Belov, V. N., and Hell, S. W. (2012). Red-emitting rhodamines with hydroxylated, sulfonated, and phosphorylated dye residues and their use in fluorescence nanoscopy. Chemistry, 18(41), 12986-12998.

Kozak, M. (1987). An analysis of 5'-noncoding sequences from 699 vertebrate messenger RNAs. Nucleic Acids Research, 15(20), 8125-8148.

Krauss, M., Kukhtina, V., Pechstein, A., and Haucke, V. (2006). Stimulation of phosphatidylinositol kinase type I-mediated phosphatidylinositol (4,5)-bisphosphate synthesis by AP-2mu-cargo complexes. Proceedings of the National Academy of Sciences of the United States of America, 103(32), 11934-11939.

Kutay, U., Hartmann, E., and Rapoport, T. A. (1993). A class of membrane proteins with a Cterminal anchor. Trends in Cell Biology, 3(3), 72-75.

Lacalle, R. A., de Karam, J. C., Martinez-Munoz, L., Artetxe, I., Peregil, R. M., Sot, J., ... Manes, S. (2015). Type I phosphatidylinositol 4-phosphate 5-kinase homo- and heterodimerization determines its membrane localization and activity. The FASEB Journal, 2371-2385.

Lajoie, M. J., Rovner, A. J., Goodman, D. B., Aerni, H., Haimovich, A. D., Kuznetsov, G., ... Isaacs, F. J. (2013). Genomically recoded organisms expand biological functions. Science, 342(6156), 357-360.

Lang, K., and Chin, J. W. (2014). Bioorthogonal reactions for labeling proteins. ACS Chemical Biology, 9(1), 16-20. 
Lang, K., Davis, L., Torres-Kolbus, J., Chou, C., Deiters, A., and Chin, J. W. (2012). Genetically encoded norbornene directs site-specific cellular protein labelling via a rapid bioorthogonal reaction. Nature Chemistry, 4(4), 298-304.

Lang, T. (2008). Imaging $\mathrm{Ca}^{2+}$-triggered exocytosis of single secretory granules on plasma membrane lawns from neuroendocrine cells. Methods in Molecular Biology, 440, 51-59.

Lavis, L. D., and Raines, R. T. (2008). Bright ideas for chemical biology. ACS Chemical Biology, 3(3), 142-155.

Lavis, L. D., and Raines, R. T. (2014). Bright building blocks for chemical biology. ACS Chemical Biology, 9(4), 855-866.

Lechene, C., Hillion, F., McMahon, G., Benson, D., Kleinfeld, A. M., Kampf, J. P., ... Slodzian, G. (2006). High-resolution quantitative imaging of mammalian and bacterial cells using stable isotope mass spectrometry. Journal of Biology, 5(6), 20.

Lepthien, S., Hoesl, M. G., Merkel, L., and Budisa, N. (2008). Azatryptophans endow proteins with intrinsic blue fluorescence. Proceedings of the National Academy of Sciences of the United States of America, 105(42), 16095-16100.

Levitus, M., and Ranjit, S. (2011). Cyanine dyes in biophysical research: the photophysics of polymethine fluorescent dyes in biomolecular environments. Quarterly Reviews of Biophysics, 44(1), 123-151.

Li, X., and Liu, C. C. (2014). Biological applications of expanded genetic codes. Chembiochem, 15(16), 2335-2341.

Li, X., Zhou, Q., Sunkara, M., Kutys, M. L., Wu, Z., Rychahou, P., ... Huang, C. (2013). Ubiquitylation of phosphatidylinositol 4-phosphate 5-kinase type I $\gamma$ by HECTD1 regulates focal adhesion dynamics and cell migration. Journal of Cell Science, 126(12), $2617-2628$.

Lichte, B., Veh, R. W., Meyer, H. E., Kilimann, M. W., Chemie, P., Bochum, R., and Bochum, W. (1992). a Novel Protein Associated With Synaptic. The EMBO Journal, 1(7), 2521-2530.

Lichtman, J. W., and Conchello, J.-A. (2005). Fluorescence microscopy. Nature Methods, 2(12), 910-919.

Link, A. J., Vink, M. K. S., Agard, N. J., Prescher, J. A., Bertozzi, C. R., and Tirrell, D. A. (2006). Discovery of aminoacyl-tRNA synthetase activity through cell-surface display of noncanonical amino acids. Proceedings of the National Academy of Sciences of the United States of America, 103(27), 10180-10185.

Link, A. J., and Tirrell, D. A. (2003). Cell surface labeling of Escherichia coli via copper(I)catalyzed [3+2] cycloaddition. Journal of the American Chemical Society, 125(37), $11164-11165$.

Liu, C. C., and Schultz, P. G. (2010). Adding new chemistries to the genetic code. Annual Review of Biochemistry, 79, 413-444.

Liu, D. R., and Schultz, P. G. (1999). Progress toward the evolution of an organism with an expanded genetic code. Proceedings of the National Academy of Sciences of the United States of America, 96(9), 4780-4785.

Liu, W., Brock, A., Chen, S., Chen, S., and Schultz, P. G. (2007). Genetic incorporation of 
unnatural amino acids into proteins in mammalian cells. Nature Methods, 4(3), 239-244.

Longmaid, H. E., Adams, D. F., Neirinckx, R. D., Harrison, C. G., Brunner, P., Seltzer, S. E., ... Geyer, R. P. (1985). In vivo ${ }^{19} \mathrm{~F}$ NMR imaging of liver, tumor, and abscess in rats. Preliminary results. Investigative Radiology, 20(2), 141-145.

Los, G. V, Encell, L. P., McDougall, M. G., Hartzell, D. D., Karassina, N., Zimprich, C., ... Wood, K. V. (2008). HaloTag: a novel protein labeling technology for cell imaging and protein analysis. ACS Chemical Biology, 3(6), 373-382.

Löschberger, A., Niehörster, T., and Sauer, M. (2014). Click chemistry for the conservation of cellular structures and fluorescent proteins: ClickOx. Biotechnology Journal, 9(5), 693697.

Lukinavičius, G., Lavogina, D., Orpinell, M., Umezawa, K., Reymond, L., Garin, N., ... Johnsson, K. (2013). Selective chemical crosslinking reveals a Cep57-Cep63-Cep152 centrosomal complex. Current Biology, 23(3), 265-270.

Lukinavičius, G., Reymond, L., D’Este, E., Masharina, A., Göttfert, F., Ta, H., ... Johnsson, K. (2014). Fluorogenic probes for live-cell imaging of the cytoskeleton. Nature Methods, 11(7), 731-733.

Lukinavičius, G., Umezawa, K., Olivier, N., Honigmann, A., Yang, G., Plass, T., ... Johnsson, K. (2013). A near-infrared fluorophore for live-cell super-resolution microscopy of cellular proteins. Nature Chemistry, 5(2), 132-139.

Meijer, M., Burkhardt, P., de Wit, H., Toonen, R. F., Fasshauer, D., and Verhage, M. (2012). Munc18-1 mutations that strongly impair SNARE-complex binding support normal synaptic transmission. The EMBO Journal, 31(9), 2156-2168.

Meldal, M., and Tomøe, C. W. (2008). Cu-catalyzed azide - alkyne cycloaddition. Chemical Reviews, 108, 2952-3015.

Merkel, L., Hoesl, M. G., Albrecht, M., Schmidt, A., and Budisa, N. (2010). Blue fluorescent amino acids as in vivo building blocks for proteins. Chembiochem, 11(3), 305-314.

Meyer, L., Wildanger, D., Medda, R., Punge, A., Rizzoli, S. O., Donnert, G., and Hell, S. W. (2008). Dual-color STED microscopy at 30-nm focal-plane resolution. Small, 4(8), 1095-1100.

Milles, S., and Lemke, E. A. (2013). What precision-protein-tuning and nano-resolved single molecule sciences can do for each other. Bioessays, 35(1), 65-74.

Milles, S., Tyagi, S., Banterle, N., Koehler, C., Vandelinder, V., Plass, T., ... Lemke, E. A. (2012). Click strategies for single-molecule protein fluorescence. Journal of the American Chemical Society, 134(11), 5187-5195.

Morin, J. G., and Hastings, J. W. (1971). Energy transfer in a bioluminescent system. Journal of Cellular Physiology, 77(3), 313-318.

Morise, H., Shimomura, O., Johnson, F. H., and Winant, J. (1974). Intermolecular energy transfer in the bioluminescent system of Aequorea. Biochemistry, 13(12), 2656-2662.

Morozova, K. S., Piatkevich, K. D., Gould, T. J., Zhang, J., Bewersdorf, J., and Verkhusha, V. V. (2010). Far-red fluorescent protein excitable with red lasers for flow cytometry and superresolution STED nanoscopy. Biophysical Journal, 99(2), L13-L15.

Muyldermans, S. (2013). Nanobodies: natural single-domain antibodies. Annual Review of 
Biochemistry, 82(1), 775-797.

Nakamura, Y., Ito, K., and Isaksson, L. A. (1996). Emerging understanding of translation termination. Cell, 87(2), 147-150.

Neumann, H. (2012). Rewiring translation - genetic code expansion and its applications. FEBS Letters, 586(15), 2057-2064.

Neumann, H., Hazen, J. L., Weinstein, J., Mehl, R. A., and Chin, J. W. (2008). Genetically encoding protein oxidative damage. Journal of the American Chemical Society, 130(12), 4028-4033.

Neumann, H., Wang, K., Davis, L., Garcia-Alai, M., and Chin, J. W. (2010). Encoding multiple unnatural amino acids via evolution of a quadruplet-decoding ribosome. Nature, 464(7287), 441-444.

Nguyen, D. P., Lusic, H., Neumann, H., Kapadnis, P. B., Deiters, A., and Chin, J. W. (2009). Genetic encoding and labeling of aliphatic azides and alkynes in recombinant proteins via a pyrrolysyl-tRNA synthetase/tRNA(CUA) pair and click chemistry. Journal of the American Chemical Society, 131(25), 8720-8721.

Nikić, I., Kang, J. H., Girona, G. E., Aramburu, I. V., and Lemke, E. A. (2015). Labeling proteins on live mammalian cells using click chemistry. Nature Protocols, 10(5), 780791.

Nikić, I., Plass, T., Schraidt, O., Szymański, J., Briggs, J. A. G., Schultz, C., and Lemke, E. A. (2014). Minimal tags for rapid dual-color live-cell labeling and super-resolution microscopy. Angewandte Chemie International Edition, 53(8), 2245-2249.

Noren, C., Anthony-Cahill, S., Griffith, M., and Schultz, P. (1989). A general method for sitespecific incorporation of unnatural amino acids into proteins. Science, 244, 182-188.

Noren, C. J., Anthony-Cahill, S. J., Suich, D. J., Noren, K. a, Griffith, M. C., and Schultz, P. G. (1990). In vitro suppression of an amber mutation by a chemically aminoacylated transfer RNA prepared by runoff transcription. Nucleic Acids Research, 18(1), 83-88.

Opazo, F., Levy, M., Byrom, M., Schäfer, C., Geisler, C., Groemer, T. W., ... Rizzoli, S. O. (2012). Aptamers as potential tools for super-resolution microscopy. Nature Methods, 9(10), 938-939.

Opazo, F., Punge, A., Bückers, J., Hoopmann, P., Kastrup, L., Hell, S. W., and Rizzoli, S. O. (2010). Limited intermixing of synaptic vesicle components upon vesicle recycling. Traffic, 11(6), 800-812.

Ormö, M., Cubitt, A. B., Kallio, K., Gross, L. A., Tsien, R. Y., and Remington, S. J. (1996). Crystal structure of the Aequorea victoria green fluorescent protein. Science, 273(5280), 1392-1395.

Osawa, S., Jukes, T. H., Watanabe, K., and Muto, A. (1992). Recent-evidence for evolution of the genetic-code. Microbiological Reviews, 56(1), 229-264.

Palmer, E., and Freeman, T. (2004). Investigation into the use of C- and N-terminal GFP fusion proteins for subcellular localization studies using reverse transfection microarrays. Comparative and Functional Genomics, 5(4), 342-353.

Parrish, A. R., She, X., Xiang, Z., Coin, I., Shen, Z., Briggs, S. P., ... Wang, L. (2012). Expanding the genetic code of Caenorhabditis elegans using bacterial aminoacyl-tRNA 
synthetase/tRNA pairs. ACS Chemical Biology, 7(7), 1292-1302.

Peter, B. J., Kent, H. M., Mills, I. G., Vallis, Y., Butler, P. J. G., Evans, P. R., and McMahon, H. T. (2004). BAR domains as sensors of membrane curvature: the amphiphysin BAR structure. Science, 303(5657), 495-499.

Phillips, G. J. (2001). Green fluorescent protein: a bright idea for the study of bacterial protein localization. FEMS Microbiology Letters, 204(1), 9-18.

Plass, T., Milles, S., Koehler, C., Schultz, C., and Lemke, E. A. (2011). Genetically encoded copper-free click chemistry. Angewandte Chemie International Edition, 50(17), 38783881.

Plass, T., Milles, S., Koehler, C., Szymański, J., Mueller, R., Wiessler, M., ... Lemke, E. A. (2012). Amino acids for Diels-Alder reactions in living cells. Angewandte Chemie International Edition, 51(17), 4166-4170.

Plotegher, N., Greggio, E., Bisaglia, M., and Bubacco, L. (2014). Biophysical groundwork as a hinge to unravel the biology of $\alpha$-synuclein aggregation and toxicity. Quarterly Reviews of Biophysics, 1, 1-48.

Polycarpo, C. R., Herring, S., Bérubé, A., Wood, J. L., Söll, D., and Ambrogelly, A. (2006). Pyrrolysine analogues as substrates for pyrrolysyl-tRNA synthetase. FEBS Letters, 580(28-29), 6695-6700.

Pott, M., Schmidt, M. J., and Summerer, D. (2014). Evolved sequence contexts for highly efficient amber suppression with noncanonical amino acids. ACS Chemical Biology, 9(12), 2815-2822.

Prasher, D. C., Eckenrode, V. K., Ward, W. W., Prendergast, F. G., and Cormier, M. J. (1992). Primary structure of the Aequorea victoria green-fluorescent protein. Gene, 111(2), 229-233.

Punge, A., Rizzoli, S. O., Jahn, R., Wildanger, J. D., Meyer, L., Schönle, A., ... Hell, S. W. (2008). 3D reconstruction of high-resolution STED microscope images. Microscopy Research and Technique, 71(9), 644-650.

Qin, J. Y., Zhang, L., Clift, K. L., Hulur, I., Xiang, A. P., Ren, B. Z., and Lahn, B. T. (2010). Systematic comparison of constitutive promoters and the doxycycline-inducible promoter. PLoS One, 5(5), 3-6.

Ramirez, D. M. O., Khvotchev, M., Trauterman, B., and Kavalali, E. T. (2012). Vtila identifies a vesicle pool that preferentially recycles at rest and maintains spontaneous neurotransmission. Neuron, 73(1), 121-134.

Remington, S. J. (2006). Fluorescent proteins: maturation, photochemistry and photophysics. Current Opinion in Structural Biology, 16(6), 714-721.

Revelo, N. H., and Rizzoli, S. O. (2015). Advanced fluorescence microscopy. Methods in Molecular Biology, 1251, 213-230.

Ries, J., Kaplan, C., Platonova, E., Eghlidi, H., and Ewers, H. (2012). A simple, versatile method for GFP-based super-resolution microscopy via nanobodies. Nature Methods, 9(6), 582-584.

Rosman, K. J. R., and Taylor, P. D. P. (1998). Isotopic compositions of the elements 1997. Pure and Applied Chemistry, 70(1), 217-235. 
Rostovtsev, V. V., Green, L. G., Fokin, V. V., and Sharpless, K. B. (2002). A stepwise huisgen cycloaddition process: copper(I)-catalyzed regioselective "ligation" of azides and terminal alkynes. Angewandte Chemie International Edition, 41(14), 2596-2599.

Rudner, L., Nydegger, S., Coren, L. V, Nagashima, K., Thali, M., and Ott, D. E. (2005). Dynamic fluorescent imaging of human immunodeficiency virus type 1 gag in live cells by biarsenical labeling. Journal of Virology, 79(7), 4055-4065.

Rust, M. J., Bates, M., and Zhuang, X. (2006). Sub-diffraction-limit imaging by stochastic optical reconstruction microscopy (STORM). Nature Methods, 3(10), 793-795.

Ryu, Y., and Schultz, P. G. (2006). Efficient incorporation of unnatural amino acids into proteins in Escherichia coli. Nature Methods, 3(4), 263-265.

Saka, S. K., Honigmann, A., Eggeling, C., Hell, S. W., Lang, T., and Rizzoli, S. O. (2014a). Multi-protein assemblies underlie the mesoscale organization of the plasma membrane. Nature Communications, 5, 4509.

Saka, S. K., Vogts, A., Kröhnert, K., Hillion, F., Rizzoli, S. O., and Wessels, J. T. (2014b). Correlated optical and isotopic nanoscopy. Nature Communications, 5, 3664.

Sakamoto, K., Hayashi, A., Sakamoto, A., Kiga, D., Nakayama, H., Soma, A., ... Yokoyama, S. (2002). Site-specific incorporation of an unnatural amino acid into proteins in mammalian cells. Nucleic Acids Research, 30(21), 4692-4699.

Sanders, J. D., Yang, Y., and Liu, Y. (1998). Differential turnover of syntaxin and SNAP-25 during synaptogenesis in cultured cerebellar granule neurons. Journal of Neuroscience Research, 53(6), 670-676.

Schägger, H. (2006). Tricine-SDS-PAGE. Nature Protocols, 1(1), 16-22.

Schägger, H., and von Jagow, G. (1987). Tricine-sodium dodecyl sulfate-polyacrylamide gel electrophoresis for the separation of proteins in the range from 1 to $100 \mathrm{kDa}$. Analytical Biochemistry, 166(2), 368-379.

Schneckenburger, H., Weber, P., Wagner, M., Schickinger, S., Richter, V., Bruns, T., ... Wittig, R. (2012). Light exposure and cell viability in fluorescence microscopy. Journal of Microscopy, 245(3), 311-318.

Sengupta, P., Jovanovic-Talisman, T., Skoko, D., Renz, M., Veatch, S. L., and LippincottSchwartz, J. (2011). Probing protein heterogeneity in the plasma membrane using PALM and pair correlation analysis. Nature Methods, 8(11), 969-975.

Senyo, S. E., Steinhauser, M. L., Pizzimenti, C. L., Yang, V. K., Cai, L., Wang, M., ... Lee, R. T. (2013). Mammalian heart renewal by pre-existing cardiomyocytes. Nature, 493(7432), 433-436.

Shaner, N. C., Patterson, G. H., and Davidson, M. W. (2007). Advances in fluorescent protein technology. Journal of Cell Science, 120(24), 4247-4260.

Shroff, H., Galbraith, C. G., Galbraith, J. A., and Betzig, E. (2008). Live-cell photoactivated localization microscopy of nanoscale adhesion dynamics. Nature Methods, 5(5), 417423.

Sieber, J. J., Willig, K. I., Kutzner, C., Gerding-Reimers, C., Harke, B., Donnert, G., ... Lang, T. (2007). Anatomy and dynamics of a supramolecular membrane protein cluster. Science, 317(5841), 1072-1076. 
Söll, D. (1988). Genetic code: enter a new amino acid. Nature, 331(6158), 662-663.

Srinivasan, G., James, C. M., and Krzycki, J. A. (2002). Pyrrolysine encoded by UAG in Archaea: charging of a UAG-decoding specialized tRNA. Science, 296(5572), 14591462.

Stadler, C., Rexhepaj, E., Singan, V. R., Murphy, R. F., Pepperkok, R., Uhlén, M., ... Lundberg, E. (2013). Immunofluorescence and fluorescent-protein tagging show high correlation for protein localization in mammalian cells. Nature Methods, 10(4), 315-323.

Staros, J. V, Bayley, H., Standring, D. N., and Knowles, J. R. (1978). Reduction of aryl azides by thiols: implications for the use of photoaffinity reagents. Biochemical and Biophysical Research Communications, 80(3), 568-572.

Staudinger, H., and Meyer, J. (1919). Über neue organische Phosphorverbindungen. Helvetica Chimica Acta, 2(1), 612-618.

Stein, A., Weber, G., Wahl, M. C., and Jahn, R. (2009). Helical extension of the neuronal SNARE complex into the membrane. Nature, 460(7254), 525-528.

Steinhauser, M. L., and Lechene, C. P. (2013). Quantitative imaging of subcellular metabolism with stable isotopes and multi-isotope imaging mass spectrometry. Seminars in Cell and Developmental Biology, 24(8-9), 661-667.

Stennett, E. M. S., Ciuba, M. A., and Levitus, M. (2014). Photophysical processes in single molecule organic fluorescent probes. Chemical Society Reviews, 43(4), 1057-1075.

Stephens, D. J., and Allan, V. J. (2003). Light microscopy techniques for live cell imaging. Science, 300(5616), 82-86.

Stöhr, K., Siegberg, D., Ehrhard, T., Lymperopoulos, K., Öz, S., Schulmeister, S., ... Herten, D.-P. (2010). Quenched substrates for live-cell labeling of SNAP-tagged fusion proteins with improved fluorescent background. Analytical Chemistry, 82(19), 8186-8193.

Stokes, G. G. (1852). On the change of refrangibility of light. No. II. Philosophical Transactions of the Royal Society of London, 142(0), 463-562.

Szpak, P., White, C. D., Longstaffe, F. J., Millaire, J. F., and Vásquez Sánchez, V. F. (2013). Carbon and nitrogen isotopic survey of northern peruvian plants: baselines for paleodietary and paleoecological studies. PLoS One, 8(1), e53763.

Szymborska, A., de Marco, A., Daigle, N., Cordes, V. C., Briggs, J. A. G., and Ellenberg, J. (2013). Nuclear pore scaffold structure analyzed by super-resolution microscopy and particle averaging. Science, 341(6146), 655-658.

Ta, H., Keller, J., Haltmeier, M., Saka, S. K., Schmied, J., Opazo, F., ... Hell, S. W. (2015). Mapping molecules in scanning far-field fluorescence nanoscopy. Nature Communications, 6, 7977.

Tajima, M., Crane, J. M., and Verkman, A. S. (2010). Aquaporin-4 (AQP4) associations and array dynamics probed by photobleaching and single-molecule analysis of green fluorescent protein-AQP4 chimeras. Journal of Biological Chemistry, 285(11), 81638170.

Takamori, S., Holt, M., Stenius, K., Lemke, E. A., Grønborg, M., Riedel, D., ... Jahn, R. (2006). Molecular anatomy of a trafficking organelle. Cell, 127(4), 831-846.

Takei, K., Slepnev, V. I., Haucke, V., and De Camilli, P. (1999). Functional partnership 
between amphiphysin and dynamin in clathrin-mediated endocytosis. Nature Cell Biology, 1(1), 33-39.

Tanaka, K. A. K., Suzuki, K. G. N., Shirai, Y. M., Shibutani, S. T., Miyahara, M. S. H., Tsuboi, H., ... Kusumi, A. (2010). Membrane molecules mobile even after chemical fixation. Nature Methods, 7(11), 865-866.

Testa, I., Wurm, C. A., Medda, R., Rothermel, E., Von Middendorf, C., Fölling, J., ... Eggeling, C. (2010). Multicolor fluorescence nanoscopy in fixed and living cells by exciting conventional fluorophores with a single wavelength. Biophysical Journal, 99(8), 2686-2694.

Thomas, B. R. (1970). The origin of the genetic code. Biochemical and Biophysical Research Communications, 40(6), 1289-1296.

Thompson, R. E., Larson, D. R., and Webb, W. W. (2002). Precise nanometer localization analysis for individual fluorescent probes. Biophysical Journal, 82(5), 2775-2783.

Toonen, R. F. G., De Vries, K. J., Zalm, R., Südhof, T. C., and Verhage, M. (2005). Munc181 stabilizes syntaxin 1, but is not essential for syntaxin 1 targeting and SNARE complex formation. Journal of Neurochemistry, 93(6), 1393-1400.

Tran, T. H. T., Zeng, Q., and Hong, W. (2007). VAMP4 cycles from the cell surface to the trans-Golgi network via sorting and recycling endosomes. Journal of Cell Science, 120(6), 1028-1041.

Tsien, R. Y. (1998). The green fluorescent protein. Annual Review of Biochemistry, 67, 509544.

Uttamapinant, C., Howe, J. D., Lang, K., Beránek, V., Davis, L., Mahesh, M., ... Chin, J. W. (2015). Genetic code expansion enables live-cell and super-resolution imaging of sitespecifically labeled cellular proteins. Journal of the American Chemical Society, 137(14), 4602-4605.

van de Linde, S., Heilemann, M., and Sauer, M. (2012). Live-cell super-resolution imaging with synthetic fluorophores. Annual Review of Physical Chemistry, 63, 519-540.

van de Linde, S., Löschberger, A., Klein, T., Heidbreder, M., Wolter, S., Heilemann, M., and Sauer, M. (2011). Direct stochastic optical reconstruction microscopy with standard fluorescent probes. Nature Protocols, 6(7), 991-1009.

van den Bout, I., and Divecha, N. (2009). PIP5K-driven PtdIns(4,5)P2 synthesis: regulation and cellular functions. Journal of Cell Science, 122(21), 3837-3850.

Verhage, M., Maia, A. S., Plomp, J. J., Brussaard, A. B., Heeroma, J. H., Vermeer, H., ... Südhof, T. C. (2000). Synaptic assembly of the brain in the absence of neurotransmitter secretion. Science, 287(5454), 864-869.

von Stetten, D., Noirclerc-Savoye, M., Goedhart, J., Gadella, T. W. J., and Royant, A. (2012). Structure of a fluorescent protein from Aequorea victoria bearing the obligate-monomer mutation A206K. Acta Crystallographica, 68(8), 878-882.

Vreja, I. C., Kabatas, S., Saka, S. K., Kröhnert, K., Höschen, C., Opazo, F., ... Rizzoli, S. O. (2015). Secondary-ion mass spectrometry of genetically encoded targets. Angewandte Chemie International Edition, 54(19), 5784-5788.

Wagner, M. (2009). Single-cell ecophysiology of microbes as revealed by Raman 
microspectroscopy or secondary ion mass spectrometry imaging. Annual Review of Microbiology, 63, 411-429.

Wang, K., Neumann, H., Peak-Chew, S. Y., and Chin, J. W. (2007). Evolved orthogonal ribosomes enhance the efficiency of synthetic genetic code expansion. Nature Biotechnology, 25(7), 770-777.

Wang, L., Brock, A., Herberich, B., and Schultz, P. G. (2001). Expanding the genetic code of Escherichia coli. Science, 292(5516), 498-500.

Wang, L., Magliery, T. J., Liu, D. R., and Schultz, P. G. (2000). A new functional suppressor tRNA / aminoacyl-tRNA synthetase pair for the in vivo incorporation of unnatural amino acids into proteins, 122(11), 5010-5011.

Wedrychowski, A., Schmidt, W. N., and Hnilica, L. S. (1986). The in vivo cross-linking of proteins and DNA by heavy metals. Journal of Biological Chemistry, 261(7), 33703376.

Wenk, M. R., Pellegrini, L., Klenchin, V. A., Di Paolo, G., Chang, S., Daniell, L., ... De Camilli, P. (2001). PIP kinase I $\gamma$ is the major PI(4,5)P2 synthesizing enzyme at the synapse. Neuron, 32(1), 79-88.

Westphal, V., and Hell, S. W. (2005). Nanoscale resolution in the focal plane of an optical microscope. Physical Review Letters, 94(14), 1-4.

Westphal, V., Rizzoli, S. O., Lauterbach, M. A., Kamin, D., Jahn, R., and Hell, S. W. (2008). Video-rate far-field optical nanoscopy dissects synaptic vesicle movement. Science, 320(5873), 246-249.

Wigge, P., Köhler, K., Vallis, Y., Doyle, C. A., Owen, D., Hunt, S. P., and McMahon, H. T. (1997). Amphiphysin heterodimers: potential role in clathrin-mediated endocytosis. Molecular Biology of the Cell, 8(10), 2003-2015.

Willig, K. I., Rizzoli, S. O., Westphal, V., Jahn, R., and Hell, S. W. (2006). STED microscopy reveals that synaptotagmin remains clustered after synaptic vesicle exocytosis. Nature, 440(7086), 935-939.

Wittig, G., and Krebs, A. (1961). Zur Existenz niedergliedriger Cycloalkine, I. Chemische Berichte, 94(12), 3260-3275.

Wolbers, F., ter Braak, P., Le Gac, S., Luttge, R., Andersson, H., Vermes, I., and van den Berg, A. (2006). Viability study of HL60 cells in contact with commonly used microchip materials. Electrophoresis, 27(24), 5073-5080.

Wombacher, R., and Cornish, V. W. (2011). Chemical tags: applications in live cell fluorescence imaging. Journal of Biophotonics, 4(6), 391-402.

Wurm, C. A., Kolmakov, K., Göttfert, F., Ta, H., Bossi, M., Schill, H., ... Hell, S. W. (2012). Novel red fluorophores with superior performance in STED microscopy. Optical Nanoscopy, 1(1), 7.

Wurm, C. A., Neumann, D., Lauterbach, M. A., Harke, B., Egner, A., Hell, S. W., and Jakobs, S. (2011). Nanoscale distribution of mitochondrial import receptor Tom 20 is adjusted to cellular conditions and exhibits an inner-cellular gradient. Proceedings of the National Academy of Sciences of the United States of America, 108(33), 13546-13551.

$\mathrm{Xu}$, K., Zhong, G., and Zhuang, X. (2013). Actin, spectrin, and associated proteins form a 
periodic cytoskeletal structure in axons. Science, 339(6118), 452-456.

Yanagisawa, T., Ishii, R., Fukunaga, R., Kobayashi, T., Sakamoto, K., and Yokoyama, S. (2008). Multistep engineering of pyrrolysyl-tRNA synthetase to genetically encode $\mathrm{N}$ (epsilon)-(o-azidobenzyloxycarbonyl) lysine for site-specific protein modification. Chemistry and Biology, 15(11), 1187-1197.

Yano, T., Teruya, K., Shirahata, S., Watanabe, J., Osada, K., Tachibana, H., .. Murakami, H. (1994). Ras oncogene enhances the production of a recombinant protein regulated by the cytomegalovirus promoter in BHK-21 cells. Cytotechnology, 16(3), 167-178.

Young, T. S., Ahmad, I., Yin, J. A., and Schultz, P. G. (2010). An enhanced system for unnatural amino acid mutagenesis in E. coli. Journal of Molecular Biology, 395(2), 361374.

Zeng, Q., Tran, T. T. H., Tan, H. X., and Hong, W. (2003). The cytoplasmic domain of Vamp4 and Vamp5 is responsible for their correct subcellular targeting: The N-terminal extension of Vamp4 contains a dominant autonomous targeting signal for the trans-Golgi network. Journal of Biological Chemistry, 278(25), 23046-23054.

Zhang, D.-S., Piazza, V., Perrin, B. J., Rzadzinska, A. K., Poczatek, J. C., Wang, M., ... Lechene, C. P. (2012). Multi-isotope imaging mass spectrometry reveals slow protein turnover in hair-cell stereocilia. Nature, 481(7382), 520-524. 


\section{APPENDIX}

A table with the sizes of the molecules in Figure 1-1 is shown below. The measurements were performed manually by Burkhard Rammner and the results are shown in the xyz coordinates, where $\mathrm{Ox}, \mathrm{Oy}$ and $\mathrm{Oz}$ indicate a horizontal, vertical and front to back direction, respectively.

Table A-1 The sizes* of the molecules in Figure 1-1

\begin{tabular}{|c|c|c|c|c|}
\hline Molecule & Ox (nm) & Oy (nm) & $\mathrm{Oz}(\mathrm{nm})$ & MW (kDa) \\
\hline Alexa647 & 2.3 & 1.8 & 0.97 & 0.85 \\
\hline Affibody & 4.06 & 2.85 & 2.63 & 6 \\
\hline $\begin{array}{l}\text { Antibody } \\
\text { (primary) }\end{array}$ & 13 & 13.6 & 7 & 150 \\
\hline $\begin{array}{l}\text { Antibody } \\
\text { (primary+secondaries) }\end{array}$ & 24 & 29 & 10.3 & 450 \\
\hline Aptamer & 6.1 & 3.25 & 2.55 & $13-15$ \\
\hline Atto647N** & 1.35 & 1.46 & 1.01 & 1 \\
\hline Fab & 7.75 & 5.4 & 5.08 & 50 \\
\hline GFP & 5.1 & 4.2 & 3.22 & 26 \\
\hline PRK & 1.63 & 0.49 & 0.48 & 0.26 \\
\hline scFv & 5.45 & 5.4 & 3.8 & 27 \\
\hline SK155 & 2.09 & 2.96 & 1.79 & 2.3 \\
\hline Star635P & 1.37 & 1.95 & 0.86 & 1 \\
\hline TriazNF1 & 2.95 & 2.9 & 2.1 & 2.5 \\
\hline $\begin{array}{l}\text { VHH } \\
\text { (nanobody) }\end{array}$ & 5.24 & 5.2 & 3.6 & $13-15$ \\
\hline
\end{tabular}

* The sizes were measured by Burkhard Rammner based on their 3D rendering from pdb files. 


\section{Appendix}

**Note that the Atto647N values are given for the carboxy form because the structure of its azide derivative is not disclosed by the producer. In constrast, the Star635P and the Alexa647N values represent the ones for the azide derivatives. 
Table A-2 Summary of investigated proteins that showed significant differences*

\begin{tabular}{|c|c|c|c|}
\hline $\begin{array}{l}\text { Protein } \\
\text { name }\end{array}$ & Localization & General function & $\begin{array}{l}\text { Significant } \\
\text { changes if FP } \\
\text { tagged }\end{array}$ \\
\hline$\beta$-actin & cytoplasm/cell cortex & cytoskeleton component & $\begin{array}{l}\text { peak intensity } \\
\text { total intensity }\end{array}$ \\
\hline amphiphysin & cytoplasm/peripheral & clathrin-mediated endocytosis & $\begin{array}{l}\text { peak intensity } \\
\text { total intensity }\end{array}$ \\
\hline Munc18-1 & $\begin{array}{l}\text { cytoplasm/cell } \\
\text { membrane }\end{array}$ & $\begin{array}{l}\text { regulates synaptic vesicle } \\
\text { docking and fusion }\end{array}$ & $\begin{array}{l}\text { peak intensity } \\
\text { total intensity }\end{array}$ \\
\hline PIPKI $\gamma$ & $\begin{array}{l}\text { cell } \\
\text { membrane/cytoplasm }\end{array}$ & $\begin{array}{l}\text { phosphorylates PtdIns } 4 \mathrm{P} \text { to } \\
\text { generate the second messenger } \\
\text { PtdIns }(4,5) \mathrm{P} 2\end{array}$ & $\begin{array}{l}\text { spot size } \\
\text { peak intensity } \\
\text { total intensity }\end{array}$ \\
\hline syntaxin 6 & $\begin{array}{l}\text { Golgi apparatus } \\
\text { (tail-anchored) }\end{array}$ & intracellular vesicle trafficking & total intensity \\
\hline VAMP4 & $\begin{array}{l}\text { trans-Golgi network } \\
\text { (tail-anchored) }\end{array}$ & $\begin{array}{l}\text { transport from early endosomes } \\
\text { to trans-Golgi network }\end{array}$ & $\begin{array}{l}\text { spot size } \\
\text { peak intensity } \\
\text { total intensity }\end{array}$ \\
\hline Vtila- $\beta$ & $\begin{array}{l}\text { small synaptic } \\
\text { vesicles } \\
\text { (tail-anchored) }\end{array}$ & vesicle transport & spot size \\
\hline
\end{tabular}

*Continued in the appendix for the rest of the proteins

The characteristics of the proteins are presented according to Uniprot (www.uniprot.org) and the references cited there. 
Table A-3 Fluorescence properties for different far-red dyes

\begin{tabular}{|c|c|c|c|c|c|}
\hline $\begin{array}{l}\text { Dye } \\
\text { (class) }\end{array}$ & $\begin{array}{l}\lambda_{\mathrm{abs}} / \lambda_{\mathrm{em}} \\
{[\mathrm{nm}]}\end{array}$ & $\begin{array}{l}\varepsilon_{\max } \\
{\left[10^{-3} \mathrm{M}^{-}\right.} \\
\left.{ }_{1} \mathrm{~cm}^{-1}\right]\end{array}$ & QY & Background & References \\
\hline $\begin{array}{l}\text { Alexa647 } \\
\text { (cyanine) }\end{array}$ & $650 / 665$ & 239 & 0.33 & low & $\begin{array}{l}\text { Campos et al., 2011; } \\
\text { Lukinavičius et al., } 2013\end{array}$ \\
\hline $\begin{array}{l}\text { Atto647N } \\
\text { (carbopyronine) }\end{array}$ & $644 / 669$ & 150 & 0.51 & high & $\begin{array}{l}\text { Stöhr et al., 2010; } \\
\text { Kolmakov et al., 2012; } \\
\text { Lukinavičius et al., } 2013\end{array}$ \\
\hline $\begin{array}{l}\text { KK114 } \\
\text { (rhodamine) }\end{array}$ & $637 / 660$ & $92^{\mathrm{w}}$ & $\begin{array}{l}0.53 \\
\mathrm{w}\end{array}$ & low & Kolmakov et al., 2010 \\
\hline $\begin{array}{l}\text { Star635 } \\
\text { (rhodamine) }\end{array}$ & $634 / 654$ & $63^{w}$ & $\begin{array}{l}0.55 \\
\mathrm{w}\end{array}$ & low & Kolmakov et al., 2010 \\
\hline $\begin{array}{l}\text { Star635P } \\
\text { (rhodamine) }\end{array}$ & $635 / 655$ & $75^{\mathrm{w}}$ & $\begin{array}{l}0.55 \\
\text { w }\end{array}$ & low & Kolmakov et al., 2010 \\
\hline $\begin{array}{l}\text { SiR-methyl } \\
\text { (Si-rhodamine) }\end{array}$ & $648 / 662$ & 100 & 0.39 & high & Lukinavičius et al., 2013 \\
\hline $\begin{array}{l}\text { SiR-carboxyl } \\
\text { (Si-rhodamine) }\end{array}$ & $645 / 661$ & 100 & 0.39 & low & Lukinavičius et al., 2013 \\
\hline
\end{tabular}

${ }^{\mathrm{w}}$ - measured in water;

Abbreviations: $\lambda_{\mathrm{abs}} / \lambda_{\mathrm{em}}-$ absorption and emission maxima wavelength, $\mathrm{QY}$ - quantum yield 
The chemical structures for the TriazNF2 NanoSIMS probe:

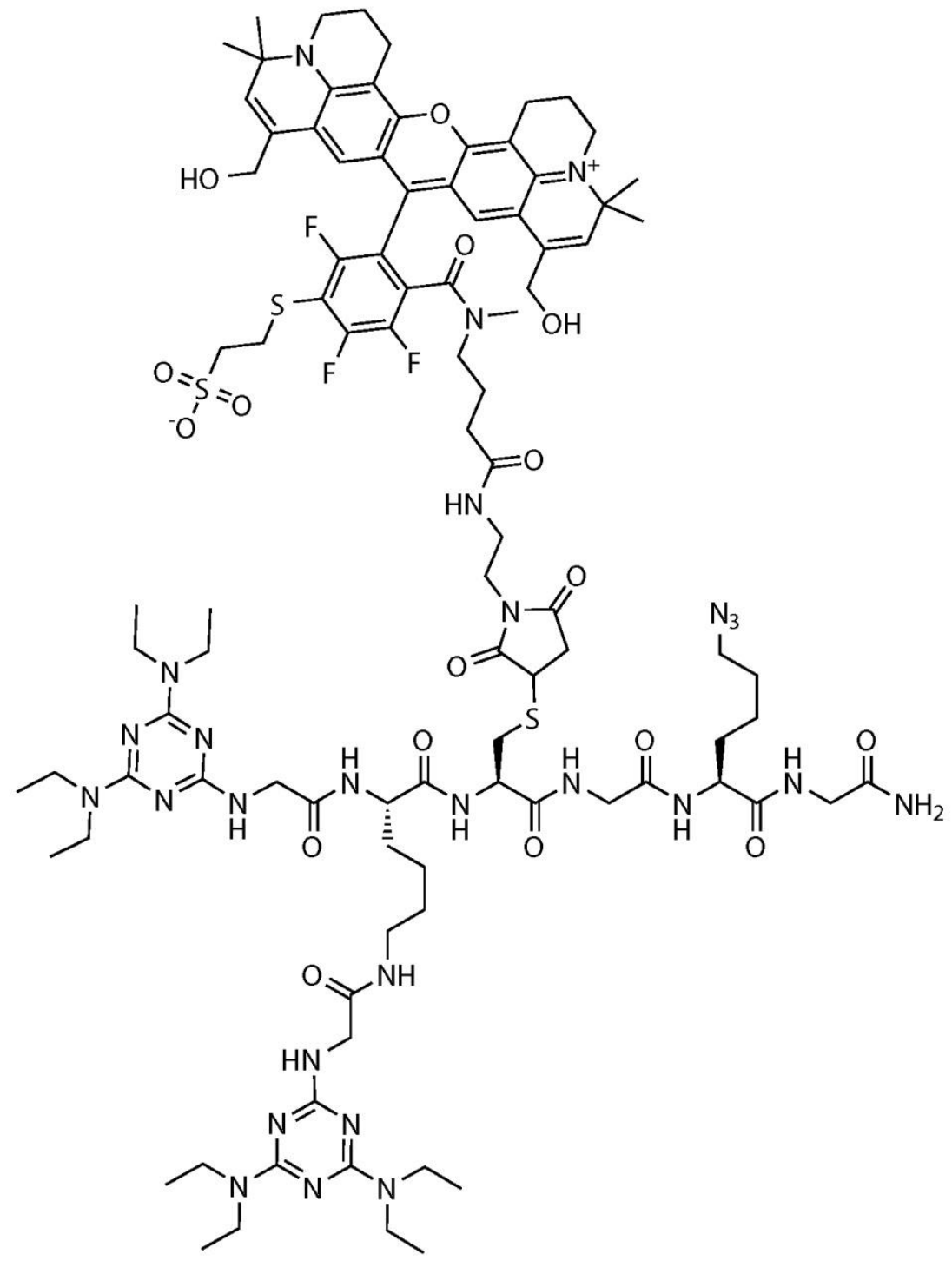

TriazNF2 
The chemical structures for the TriazNF3 NanoSIMS probe:

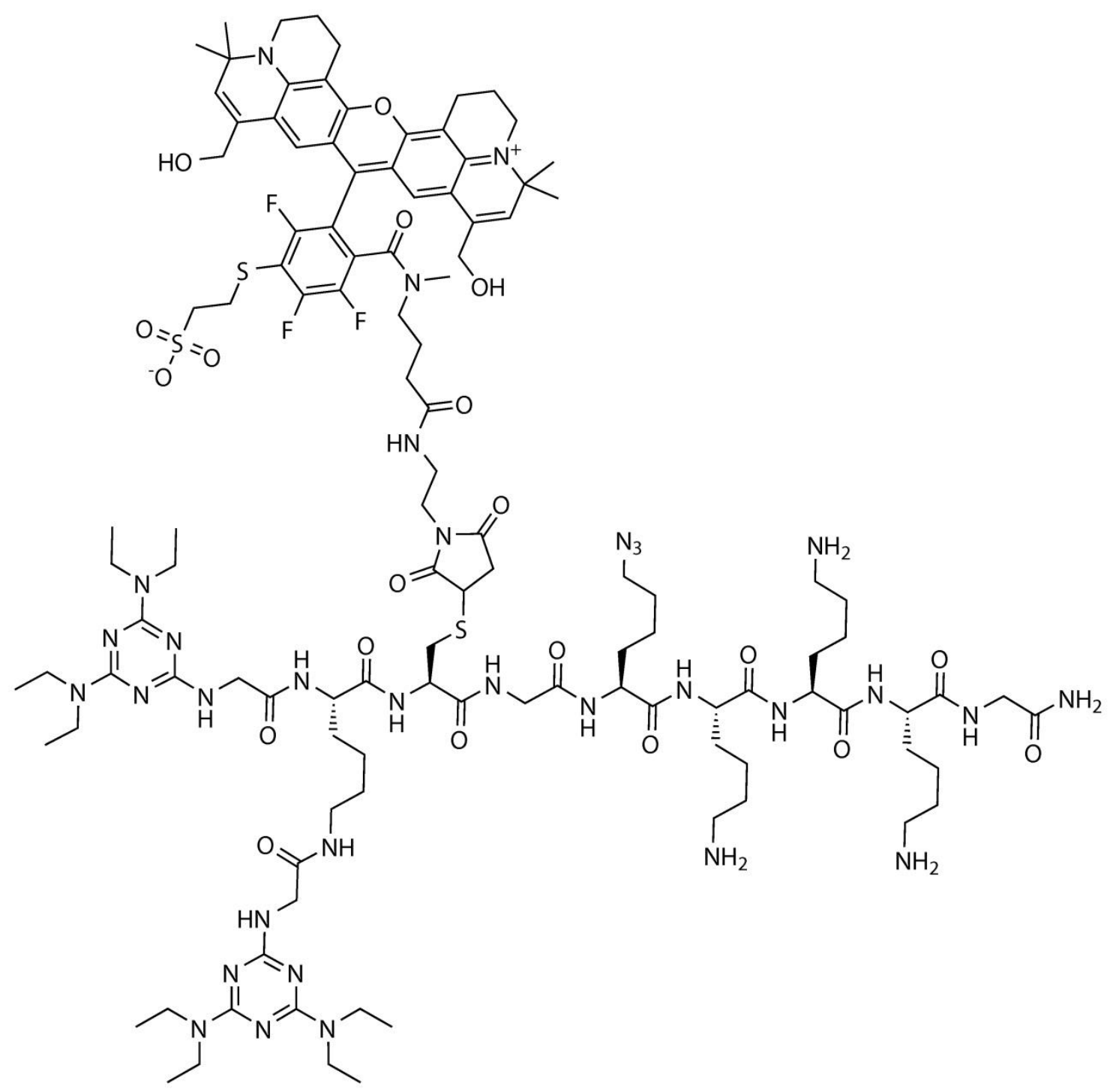

TriazNF3 


\section{CURRICULUM VITAE}

\section{PERSONAL INFORMATION}

Name: $\quad$ Ingrid-Cristiana

Surname: $\quad$ Gebura-Vreja

Place of birth: $\quad$ Râmnicu-Vâlcea, Romania

Date of birth: $\quad$ September $26^{\text {th }}, 1987$

E-mail: $\quad$ ivreja@gwdg.de

\section{EDUCATION}

10/2011 - 9/2015 Doctoral work

European Neuroscience Institute, Göttingen and at the Institute of Neuro- and Sensory Physiology, University Medical Center, Göttingen, Germany

10/2011 - 3/2012 MSc Degree in Molecular Biology

European Neuroscience Institute, Göttingen, Germany

2010 - 9/2015 Molecular Biology MSc/PhD Program, International Max Planck Research School, University of Göttingen, Germany

$2007-2010 \quad$ Bachelor of Science in Biochemistry

Faculty of Biology, University of Bucharest, Romania

2003 - $2004 \quad$ High School Diploma

"Matei Basarab" National Computer Science College, Râmnicu-Vâlcea, Romania 


\section{RESEARCH EXPERIENCE}

10/2011 - 9/2015 PhD project in the lab of Prof. Dr. Silvio Rizzoli

European Neuroscience Institute and University Medical Center

Göttingen, Germany

"Unnatural amino acids as minimal tags for investigating cellular organization and turnover"

10/2011 - 3/2012 MSc project in the lab of Prof. Dr. Silvio Rizzoli

European Neuroscience Institute, Göttingen, Germany

"Testing the potential role of super-abundant SNARE molecules in the plasma membrane"

5/2011 - 06/2011 Lab rotation with Dr. John Chua

Max Planck Institute for Biophysical Chemistry, Göttingen,

Germany

"Bimolecular complementation studies of cargo-motor protein interactions"

2/2011 - 04/2011 Lab rotation with Prof. Dr. Matthias Dobbelstein

Göttingen Center for Molecular Biosciences (GZMB), Göttingen,

Germany

"The role of miR-22 in tumor suppression"

1/2011 - 09/2010 Lab rotation with Prof. Dr. Silvio Rizzoli

European Neuroscience Institute, Göttingen, Germany

"Techniques to Investigate Synaptic Vesicle Recycling"

5/2009-09/2010 Bachelor project

Institute of Biochemistry, Bucharest, Romania

"Enzymatic assays of the protein tyrosine phosphatase D1 catalytic domain mutants obtained by site-directed mutagenesis"

\section{AWARDS AND SCHOLARSHIPS}

2015 Participation in the Lindau Nobel Laureate Meeting

2012 - 2015 Dorothea Schlözer PhD Fellowship

2014 GGNB Travel Grant

$2013 \quad 3^{\text {rd }}$ Poster Prize at the Cologne Excellent Women in Science Symposium

2010 - 2011 Stipend of the Excellence Foundation for the Promotion of the Max Planck Society

2007 - 2010 Olympic Merit Scholarship awarded by the Romanian Government

2007 - 2010 Sindan Pharma Scholarship

2007 Bronze Medal at the $39^{\text {th }}$ International Chemistry Olympiad (IChO), Moscow, Russia 

Minsk, Belarus

\section{SCIENTIFIC COMMUNICATION}

- $15^{\text {th }}$ EuCheMS International Conference on Chemistry and the Environment; Highresolution imaging and correlative analyses in life sciences down to sub nanometre scale, Leipzig, 20-24 ${ }^{\text {th }}$ Septmeber 2015

- Assembly and Disassembly of the Neurvous System, 19-2 ${ }^{\text {st }}$ January, Rehovot, Israel

- $1^{\text {st }}$ Society for Neuroscience Annual Meeting, 15-19 $9^{\text {th }}$ November, Washington, DC, USA

- $1^{\text {st }}$ Cologne Excellent Women in Science Symposium, 6-7 $7^{\text {th }}$ July 2013, Köln, Germany

- $5^{\text {th }}$ Neurizons Meeting, 22-25 ${ }^{\text {th }}$ May 2013, Göttingen, Germany

- $9^{\text {th }}$ Horizons in Molecular Biology Symposium, 8-11 ${ }^{\text {th }}$ October 2012, Göttingen, Germany

- $1^{\text {st }}$ Women's Careers and Networks Conference, $3^{\text {rd }}$ November 2011, Göttingen, Germany

- $7^{\text {th }}$ Horizons in Molecular Biology Symposium, 27-30 ${ }^{\text {th }}$ September 2010, Göttingen, Germany

\section{WORK AND TEACHING EXPERIENCE}

-28/11/2012: $\quad$ Practical course for Neuroscience MSc/PhD Program. International Max Planck Research School, Göttingen, Germany

-12/12/2012: High-resolution microscopy workshop. Horizons in Molecular Biology PhD Symposium, Göttingen, Germany

\section{ORGANIZATION OF ACADEMIC EVENTS}

- Horizons in Molecular Biology Symposium, Göttingen, Germany $\left(9^{\text {th }}\right.$ and $10^{\text {th }}$ edition $)$

- Women's Careers and Networks Conference, Göttingen, Germany ( $2^{\text {nd }}$ and $3^{\text {rd }}$ edition $)$ 

"Îmi învățam cuvintele să iubească

le arătam inima

și nu mă lăsam până când silabele lor

Nu începeau să bată (...)

numai s-ajungă mai iute la destinație,

numai ca să le-nvăț cum se transportă lumea,

de la ea însăși,

la ea însăși."

Nichita Stănescu, Ars poetica, 1965
"I taught my words to love,

I showed them my heart

and would not give up until their syllables

did not start to beat (...)

only so they could cross faster,

only so they would learn how to transport the world,

from itself,

to itself." 